SÉRIE GÉNÉALOGIES coordonnée par Anne-Marie Bertrand

BIBLIOTHËQUE PUBLIQUE ET PUBLIC LIBRARY :

ESSAI DE GÉNÉALOGIE COMPARÉE

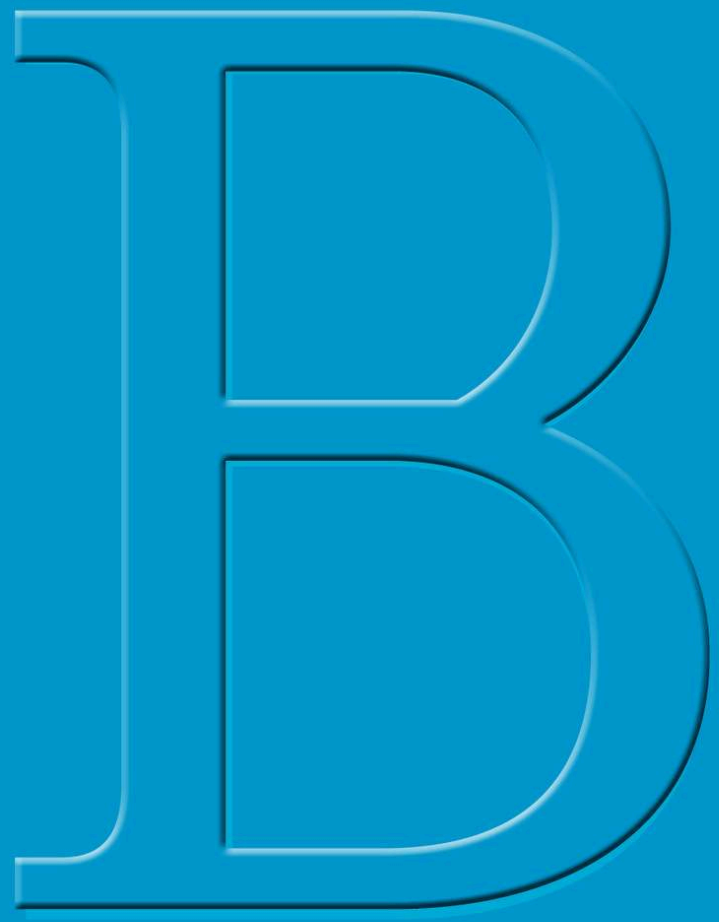




\section{Bibliothèque publique et Public Library}

Essai d'une généalogie comparée

\section{Anne-Marie Bertrand}

DOI : 10.4000/books. pressesenssib.897

Éditeur : Presses de l'enssib

Année d'édition : 2010

Date de mise en ligne : 4 avril 2017

Collection : Papiers

ISBN électronique : 9782375460436

\section{oponatibito}

http://books.openedition.org

\section{Édition imprimée}

ISBN : 9782910227784

Nombre de pages : 232

\section{Référence électronique}

BERTRAND, Anne-Marie. Bibliothèque publique et Public Library: Essai d'une généalogie comparée.

Nouvelle édition [en ligne]. Villeurbanne : Presses de l'enssib, 2010 (généré le 01 février 2021).

Disponible sur Internet : <http://books.openedition.org/pressesenssib/897>. ISBN : 9782375460436.

DOI : https://doi.org/10.4000/books.pressesenssib.897.

(c) Presses de l'enssib, 2010

Conditions d'utilisation :

http://www.openedition.org/6540 
SÉRIE GÉNÉALOGIES coordonnée par Anne-Marie Bertrand

BIBLIOTHËQUE PUBLIQUE ET PUBLIC LIBRARY :

ESSAI DE GÉNÉALOGIE COMPARÉE

Anne-Marie Bertrand

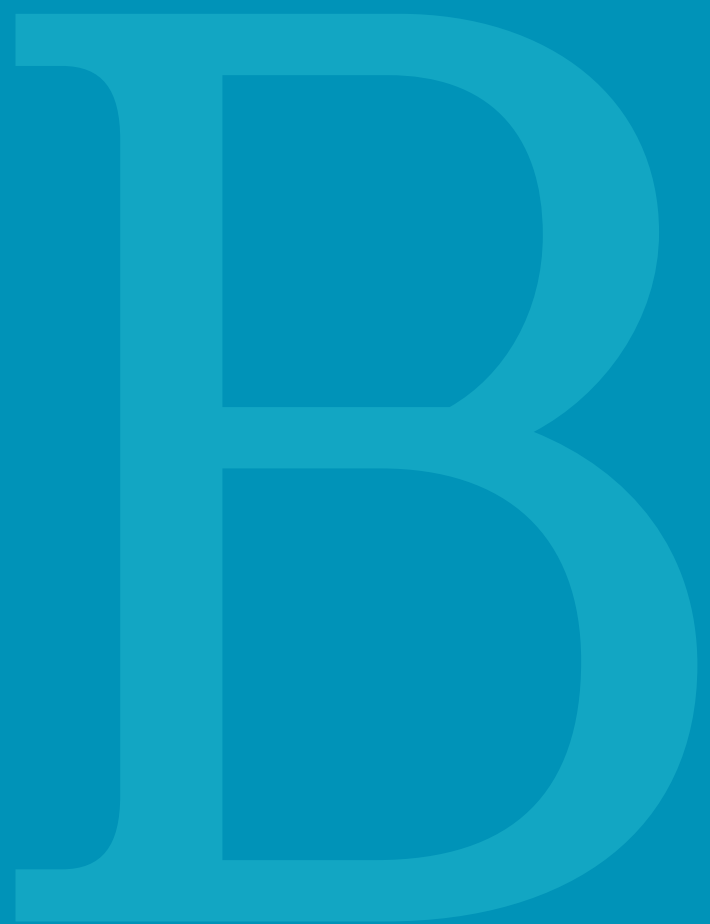


BIBLIOTHEQQUE PUBLIQUE ET PUBLIC LIBRARY :

ESSAI DE GÉNÉALOGIE COMPARÉE 


\section{PAPIERS
CLAIRE ROCHECTION DE
CLAIRE-MOIGNE}

SÉRIE GÉNÉALOGIES

coordonnée par Anne-Marie Bertrand

Cette série de trois ouvrages s'interroge sur les fondements de l'identité de la bibliothèque publique.

Le premier «Quel modèle de bibliothèques ? », paru en 2008, abordait la question du modèle de bibliothèque, son élaboration, sa diffusion, ses composantes, son évolution.

Le deuxième "Bibliothèque publique et Public Library » porte sur une analyse comparée des éléments fondateurs de la bibliothèque publique aux ÉtatsUnis et en France.

Le troisième (parution 2010) se livrera à un exercice de prospective, questionnant l'avenir et les évolutions possibles de la bibliothèque.

\section{PRESSES DE L'enssib}

École nationale supérieure des sciences de l'information et des bibliothèques 17-21 boulevard du 11 novembre 1918 69623 Villeurbanne Cedex Tél. 0472444343 - Fax 0472444344 < http://www.enssib.fr/presses > 
B. BIBLIOTHÈQUE PUBLIQUE ET PUBLIC LIBRARY : ESSAI DE GÉNÉALOGIE COMPARÉE

Anne-Marie Bertrand 
Bibliothèque publique et Public Library [Texte imprimé] : essai de généalogie comparée / Anne-Marie Bertrand. - Villeurbanne : Presses de l'enssib, cop. 2010. - 1 vol. ; 23 cm. (Collection Papiers. Série Généalogies).

ISBN 978-2-910227-78-4

Rameau :

Bibliothèques publiques - - France

Bibliothèques publiques - - États-Unis

Bibliothéconomie - - Études comparatives

Dewey : $027.4 \mathrm{~s}$

(c) enssib, 2010 
«Quel pédant inventa le mot Bibliothèque, laissant le mot français Librairie aux Anglais?»

Eugène Morel, La Librairie publique (Exergue)

Librairie Armand Colin, 1910

«Les USA, c'est une tranche de continent. Les USA, c'est un ensemble de sociétés de portefeuille, de regroupements de syndicats, une liasse de lois reliées en cuir, un réseau de radiodiffusion, une chaîne de salles de cinéma, une colonne de cotations boursières

effacées et réécrites par un garçon de la Western Union sur un tableau noir, une bibliothèque publique pleine de vieux journaux et de manuels d'histoire éculés couverts de notes indignées griffonnées au crayon dans les marges. Les USA, c'est la plus grande val-

lée fluviale du monde, bordée de montagnes et de collines. Les

USA, c'est une clique de fonctionnaires arrogants avec de trop nombreux comptes en banque. Les USA, ce sont beaucoup d'hommes enterrés au cimetière d'Arlington. Les USA, ce sont les lettres à la fin d'une adresse lorsqu'on est loin de chez soi. Mais surtout, les USA, ce sont les paroles des gens. »

John Dos Passos, USA (Introduction)

Gallimard, 2002 (Quarto) 

Préambules

Chapitre I. Voisinages ............................................................... 33

L'influence américaine sur les bibliothèques de France ... 36

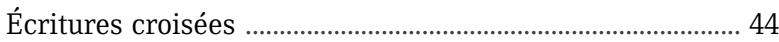

Un voisinage lointain ................................................................. 46

Chapitre II. Deux histoires, deux héritages ....................... 53

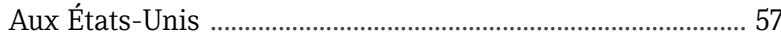

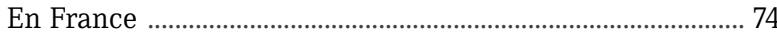

Chapitre III. Pouvoirs et bibliothèques ................................ 93

Les bibliothèques et le pouvoir central .................................... 96

Le pouvoir à l'échelon local ................................................... 108

Amis, alliés et contre-pouvoirs ................................................ 119

Chapitre IV. Groupes professionnels et associations .. 133

Des organisations ........................................................................ 134

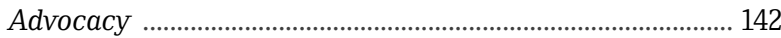

Deux combats exemplaires .................................................... 146

Chapitre V. Deux modèles de bibliothèque ..................... 159

Un socle commun ....................................................................... 160

Les bibliothèques et leurs publics ........................................... 161

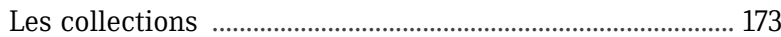

L'image de la bibliothèque ......................................................... 184

Conclusion 
Bibliographie 205

Sigles 212

Annexes 214

Library Bill of Rights 214

Déclaration des droits en bibliothèque 215

The Freedom to Read Statement 216

Déclaration sur le droit de lire 217

12 Ways Libraries Are Good for the Country 224

Douze raisons pour lesquelles les bibliothèques sont bonnes pour le pays 225 


\section{INTRODUCTION}

Q ue la bibliothèque soit un objet scientifique, voilà une assertion aujourd'hui largement acceptée ${ }^{1}$. Comme objet d'histoire, elle est au confluent de l'histoire politique, de l'histoire sociale et de l'histoire culturelle. Car elle est à la fois une institution (un établissement public), un objet social (dédié au partage du savoir et de la culture) et un artefact (construit sur un héritage, un projet et un environnement spécifiques). C'est au regard de ce dernier élément que se situe l'axe de ce travail. En effet, l'objet « bibliothèque publique », dans sa déclinaison française, est souvent décrit comme une donnée intangible, dans un " déjà là » qui semble surgi de nulle part - ou de la pure volonté kantienne, c'est-à-dire, ici, jacobine.

Mon propos est de chercher de nouveaux éléments d'intelligibilité de l'histoire récente des bibliothèques publiques en France en considérant, au contraire, que le modèle adopté n'est pas un objet surgi uniquement de l'expertise étatique mais porte le poids d'héritages séculaires, d'influences étrangères et de débats politiques sur la place de la bibliothèque dans l'espace public - ici considéré comme un espace de la délibération où se confrontent pouvoirs et contre-pouvoirs.

Cet exercice d'histoire culturelle, je le place sous le patronage d'Eugène Morel, lui qui plaidait déjà il y a un siècle pour l'adoption, en France, de la « librairie publique $»^{2}$. Formule que rappelle Michel Melot : « Les bibliothécaires français savent tout ce qu'ils doivent aux États-Unis : le principe même de la "Public Library" qu'Eugène Morel tenta d'imposer en France sous le nom de "librairie publique". ${ }^{3}$ "

1. Cet ouvrage est la version allégée et mise à jour de « De la librairie publique : petit essai d'histoire culturelle sur les bibliothèques publiques en France et aux États-Unis depuis 1945 », travail présenté pour l'habilitation à diriger des recherches, à l'université Paris 1 en 2006.

2. «Au mois d'avril 1910, quelques bibliothécaires [...] se réunirent et causèrent. Ils étaient d'accord qu'une conception nouvelle des bibliothèques était née et que le mot même de bibliothèques ne convenait plus, qu'il fallait un terme spécial pour désigner l'invention neuve, le bureau de documents de la vie courante, le centre de lecture urbaine et rurale, qui préside au prêt dans la ville et dans les campagnes, lieu de réunion aussi, monument splendide, la basilique de la cité moderne, le lycée spécial de l'instruction personnelle, l'école de recherche et d'initiative, qui aide l'école, aide le lycée, remplace l'ancienne étude, et au lieu de se clore pour l'élève quand celui-ci est à même de profiter de son instruction, s'ouvre plus grande à lui, l'accompagne dans la vie, dans la vie active où l'on ne cesse jamais d'apprendre. Le mot de Librairie publique, qu'on trouve sur ce volume, fut choisi à une de ces réunions. » (Eugène Morel, La Librairie publique, Armand Colin, 1910, p. 315).

3. Préface à Philippe Cantié. Au nom de l'antiterrorisme : les bibliothécaires américains face à l'USA Patriot Act. Presses de l'enssib, 2006, p. 9. 
Le présent ouvrage se veut donc une réflexion critique sur le modèle de bibliothèque publique en France. Modèle aujourd'hui doublement interrogé : sur sa réussite ambiguë, incomplète voire interrompue, et sur son originalité durable au sein des bibliothèques du monde.

Réussite ambiguë. Pourquoi ? Les bibliothèques municipales françaises ont connu, depuis les années 1970, un développement spectaculaire, quantitativement comme qualitativement. Les indices de moyens et d'activité ont été multipliés par 8 ou 10 en 30 ans $^{4}$, les bibliothèques sont devenues médiathèques ${ }^{5}$, elles sont aujourd'hui couramment désignées comme le service culturel de base dans une commune.

Cette success story (Pascal Ory) n'appellerait pas d'être revisitée si elle avait perduré. Or, depuis le début des années 1990, le développement s'est essoufflé, ralenti, arrêté et, même si certains facteurs continuent de croître (notamment le nombre des bibliothèques et la surface de leurs bâtiments), un élément nouveau est apparu : l'interrogation sur leur fréquentation ${ }^{6}$. Cette interrogation reflète l'inquiétude des élus comme des bibliothécaires sur l'avenir de ces établissements et, me semble-t-il, un doute sur leur légitimité et sur le bien-fondé des investissements dont ils ont bénéficié.

Au-delà de la fréquentation des bibliothèques municipales, dont la mesure même semble difficile, le constat perdure d'un écart immense entre l'activité des bibliothèques en France et dans les pays anglo-saxons. Le nombre de bibliothèques municipales, en France, était de 930 en 1980, aux États-Unis, les Public Libraries étaient 8037 en 1978. Le personnel travaillant dans ces bibliothèques représentait 7169 emplois en France en 1980, aux États-Unis 86707 en 1978 7 . La proportion de la population fréquentant une bibliothèque publique était de $10 \%$ en France en 1980 et

4. Sur ce développement, je me permets de renvoyer à Anne-Marie Bertrand. Les villes et leurs bibliothèques municipales : légitimer et décider (1945-1985). Éditions du Cercle de la Librairie, 1999, p. 112122.

5. François Rouet. La grande mutation des bibliothèques municipales. ministère de la Culture, 1998.

6. Après 20 ans de progression continue, les statistiques ministérielles indiquent ainsi 6640000 inscrits dans les bibliothèques municipales en 1998, 6582000 en 1999. Depuis, les chiffres continuent à stagner. La réalité même de cette stagnation est discutée (cf. " Mais à quoi servent vraiment les bibliothèques municipales ? », débat dans le Bulletin des bibliothèques de France, t. $50, \mathrm{n}^{\circ} 2,2005$, p. 58-72). Une enquête nationale menée en 2005 semble démentir la réalité de la stagnation - ou la nuancer : elle montre que si le nombre des usagers inscrits est en diminution, le nombre des fréquentants non inscrits est en augmentation (Bruno Maresca, Christophe Evans, Françoise Gaudet. Les bibliothèques municipales au tournant d'internet. BPI, 2007).

7. Bowker Annual of Library and Book Trade Information, 1981. Rappelons que l'écart de population est (en chiffres ronds) de 1 à 4 : en logique purement arithmétique, les chiffres français devraient être 4 fois moins élevés que les chiffres américains et non pas 9 ou 10 fois... 
de $50 \%$ aux États-Unis. Le même écart se constate avec la Grande-Bretagne et les pays scandinaves et il se maintient aujourd'hui : $18 \%$ d'inscrits dans les bibliothèques municipales en France, 58 \% en Grande-Bretagne, $60 \%$ en Suède, $66 \%$ aux États-Unis.

Comment comprendre cet écart ? Une analyse technique ne peut suffire à en rendre compte. Ces chiffres interrogent sur la place de la bibliothèque dans la société, son utilité, sa nécessité. Ils amènent donc à étudier à nouveaux frais cette histoire. Peut-on envisager une nouvelle grille d'analyse ? Derrière la lisse (mais non linéaire) histoire du développement des bibliothèques municipales, peut-on découvrir des failles, des décalages, des malentendus qui pourraient expliquer ces aléas statistiques ? Il me semble que cette entreprise est possible, à deux conditions : un retour sur le modèle de bibliothèque mis en œuvre ; une approche résolument comparatiste.

\section{UNE CRITIQUE DU MODÈLE DE BIBLIOTHĖQUE PUBLIQUE}

Conçue, attendue, espérée dès le début du $\mathrm{xx}^{\mathrm{e}}$ siècle, la bibliothèque publique d'Eugène Morel, d'Henri Vendel, de Michel Bouvy se définit principalement par des considérations techniques ou scientifiques : l'ampleur des collections, le libre accès à l'information, la fin du primat de la culture savante, la qualité des bâtiments, la qualification des personnels, etc. Le substrat politique qui sous-tend ce modèle est celui de l'ouverture à tous, c'est pourquoi on a pu l'appeler un modèle " démocratique ", qui vise l'extension (jamais achevée) du nombre de ses usagers.

La bibliothèque pour toute la population, « des livres partout, pour tous », est construite sur une double critique. D'une part, la critique de la bibliothèque savante, de sa fermeture sur elle-même, de son attention portée aux collections plutôt qu'aux usagers. D'autre part, la critique de la bibliothèque populaire, sans qualités et sans ambition ${ }^{8}$. Le modèle conçu en opposition à ce double héritage met l'usager au cœur de ses préoccupations : "Le souci des lecteurs prend le pas sur le souci des livres ${ }^{9}$ », « La maison du livre est devenue la maison du lecteur », entendon fréquemment. Cependant, le lecteur n'y est pas chez lui. Il y est, encore

8. En un sens, on pourrait considérer cette position comme malrucienne, puisqu'elle est parallèle à la double condamnation de Malraux, celle du système des beaux-arts et celle de la bonne volonté de l'éducation populaire.

9. Anne-Marie Chartier, Jean Hébrard. Discours sur la lecture (1880-2000). BPI-Fayard, 2000. 
trop souvent, mal accueilli, simplement toléré, voire considéré comme un intrus, qu'il s'agisse en particulier des étudiants ou des classes moyennes ${ }^{10}$.

Par ailleurs, le modèle de bibliothèque publique aujourd'hui mis en œuvre est un modèle qui a été soutenu et diffusé par le système politicoadministratif au niveau national. Ce n'est que dans les années 1970 que les villes se sont impliquées dans le développement de leurs bibliothèques. À l'instigation de l'État. L'offre de bibliothèque a précédé la demande de bibliothèque (c'est un constat fréquent dans le domaine culturel).

Mon hypothèse, le fil rouge de cette exploration, est qu'il y a un hiatus intrinsèque, essentiel, entre les bibliothèques et la population qu'elles ont à desservir et que cette origine fragilise le modèle de bibliothèque, qui n'est pas enraciné dans le terreau local.

Une des conséquences majeures de cet état de fait est que la question du pouvoir, de la responsabilité, de la décision au sujet de la bibliothèque est une question non traitée, qui n'a même pas de visibilité, un trou noir. D’où les questions récurrentes, toujours renaissantes, de la légitimité des bibliothèques publiques, de leur place dans l'espace public, de l'articulation entre le champ du politique et le champ du professionnel ${ }^{11}$, de l'articulation entre le savoir du professionnel et les attentes de la population (de l'offre et de la demande), de la gratuité ou de la tarification des services (qui prend en charge le service ?), etc. ${ }^{12}$

Réinterroger l'histoire des bibliothèques à la lumière de leurs relations aux pouvoirs et aux contre-pouvoirs ${ }^{13}$ me semble donc avoir du sens. D'autant plus si cette interrogation s'étend à l'exemple d'un pays étranger, les États-Unis d’Amérique.

\section{UNE ANALYSE COMPARÉE DES BIBLIOTHĖQUES PUBLIQUES AMÉRICAINES}

Sortir des lieux convenus du discours franco-français est le premier objectif de cette tentative comparatiste. Prendre de la distance, décentrer le

10. Anne-Marie Bertrand. « Le peuple, le non-public et le bon public : les publics des bibliothèques et leurs représentations chez les bibliothécaires ». Le(s) public(s) de la culture, sous la dir. d'Olivier Donnat et Paul Tolila. Presses de Sciences Po, 2003.

11. Qui a connu son apogée dans les années 1990, dans les municipalités conquises par le Front national.

12. Pour une analyse du modèle de bibliothèque publique " à la française », on se reportera également à Quel modèle de bibliothèque ? sous la dir. d'Anne-Marie Bertrand, Presses de l'enssib, 2008 (Papiers).

13. L'État, les élus, les citoyens, les usagers, les associations, etc. 
questionnement. Car si l'une de mes interrogations de départ est la mise en œuvre du modèle de bibliothèque publique, il est nécessaire d'interroger ce modèle en le comparant. La comparaison avec le modèle anglosaxon s'impose. C'est là (en Angleterre) qu'est né le Library Movement au XIX ${ }^{e}$ siècle, rapidement adopté et adapté par les jeunes États-Unis et par les pays de l'Europe du Nord. Au sein de la famille anglo-saxonne, j'ai choisi de privilégier l'exemple américain.

Parce que les bibliothécaires français, entre les deux guerres, ont été accompagnés, aidés, encouragés par les bibliothécaires américains. Parce que cela me permet de comparer l'exemple français non seulement à un autre modèle (allemand ? scandinave ? en tout cas, pas un exemple latin, où l'histoire des bibliothèques est trop voisine de celle des bibliothèques françaises pour en être éclairante) mais à celui qui a été importé en France. Ce qui est en question est, alors, non seulement l'application d'un modèle, non seulement la comparaison avec un autre modèle, mais le transfert culturel qu'est l'importation de ce modèle. Transfert, analyse Anne-Marie Thiesse, qui " permet l'élaboration de la différence dans le cadre d'un modèle général de référence ${ }^{14} »$.

Éclairer la problématique de départ par la comparaison avec le modèle américain est donc le second objectif de cette tentative comparatiste. En effet, la situation des bibliothèques américaines est assez éloignée de celle des bibliothèques françaises pour y apporter un éclairage utile ; pas assez éloignée pour que les différences soient réduites au rang d'étrangetés pittoresques. Pour le dire autrement, il s'agit de deux pays démocratiques, qui voient dans leurs bibliothèques un outil démocratique - mais, pour autant, leurs bibliothèques publiques ont une fréquentation incomparable (aujourd'hui $25 \%$ de la population en France, $66 \%$ aux ÉtatsUnis), des fonctions différentes et un rapport au(x) pouvoir(s) très différent et qui fait sens.

Aux États-Unis, les citoyens, les contribuables, les usagers ont les outils, les structures, les moyens de se faire entendre, voire de décider ce qu'ils veulent faire de leurs bibliothèques publiques. Le rapport au pouvoir local y a toujours été plus fort qu'en France. Le pouvoir central (fédéral) y est beaucoup plus lointain - sans être absent. Comment dire plus clairement les choses que par cette brève comparaison : dans les débuts du développement des bibliothèques publiques en France, les bibliothé-

14. « L'histoire culturelle est-elle une histoire nationale ?». L'histoire culturelle du contemporain, sous la dir. de Laurent Martin et Sylvain Venayre. Nouveau Monde Éditions, 2005, p. 122. 
caires attendaient de l'État une loi obligeant les villes à créer une bibliothèque ; aux États-Unis, les états ont promulgué des lois autorisant les villes à créer des bibliothèques et à lever des impôts pour les faire vivre.

L'analyse de ces différences en elles-mêmes mais aussi l'analyse de la fidélité (ou non) dans le transfert culturel qu'est l'adoption du modèle de la Public Library doivent permettre d'éclairer l'histoire contemporaine des bibliothèques en France. Ce qui m'intéresse prioritairement, c'est de savoir comment le modèle américain a été adopté en France et en quoi les infidélités de cette appropriation nous éclairent sur l'histoire culturelle et politique des bibliothèques.

Une des difficultés de cette entreprise est la grande disparité des bibliothèques publiques aux États-Unis, non seulement par leur statut, bibliothèques communales, de comté, d'état, mais aussi par leur taille et leur activité. Cette difficulté doit être assumée, et n'obère pas la validité du raisonnement. Une autre difficulté est que l'histoire des bibliothèques publiques aux États-Unis est (étrangement) peu scientifique. Les ouvrages classiques de Jesse Shera ${ }^{15}$ et de Sidney Ditzion ${ }^{16}$ sur la naissance et les premiers développements du Library Movement au XIXe siècle n'ont pas d'équivalent pour le $\mathrm{xx}^{\mathrm{e}}$ siècle. L'histoire contemporaine est trop souvent composée soit de monographies locales (qui ont fait florès à l'occasion du centenaire de la création de nombreuses bibliothèques publiques, dans les années 1970 ou 1980) soit d'une approche hagiographique du rôle des bibliothèques et des bibliothécaires ${ }^{17}$. Cette piété professionnelle, que l'on retrouve d'ailleurs à l'identique côté français, est critiquée par certains historiens des bibliothèques, qui s'appellent eux-mêmes revisionnists historians et mettent en doute la geste héroïque et linéaire des bibliothèques américaines depuis 150 ans, ce " continuum de Jefferson ou Jackson à Roosevelt ${ }^{18} »$ (Michael Harris). Ancienne, peut-être magnifiée, cette histoire est cependant le terreau où sont nées les bibliothèques-médiathèques françaises d'aujourd'hui.

15. Foundations of the Public Library: The Origins of the Public Library Movement in New England (16291855). The Shoe String Press, 1965.

16. Arsenals for a Democratic Culture. Chicago, ALA, 1947.

17. John C. Colson analyse la nombreuse production comme une étude des bibliothèques plutôt que comme une histoire des bibliothèques ("The writing of American library history has been transformed into the study of American libraries and librarianship"), John C. Colson, "The Writing of American Library History (1876-1976)”, Library Trends, July 1976. Numéro (à vrai dire décevant) qui marquait le centenaire de la création de l'ALA.

18. Michael H. Harris. "Antiquarianism, Professional Piety and Critical Scholarship in Recent American Library Historiography”. Journal of Library History, Winter 1978. 
Cette généalogie offre le grand intérêt de permettre une comparaison historique à deux moments distincts : d'une part, le moment de la matrice du modèle, au XIX ${ }^{\mathrm{e}}$ siècle aux États-Unis, pendant les six premières décennies du Xx $x^{\mathrm{e}}$ siècle pour la France ; d'autre part, le temps présent, c'est-àdire le deuxième $x x^{\mathrm{e}}$ siècle pour les deux pays. On pourra, ce faisant, observer comment la communauté initiale de principes et d'objectifs diverge vers deux réalités aujourd'hui différentes. Ma troisième hypothèse, donc le troisième fil de cet ouvrage, est qu'une lecture infidèle, une compréhension décalée, une appropriation insuffisante du modèle de la « Public Library » et de ses fondements ont mené les bibliothèques publiques françaises dans une voie où l'activité technique s'est aujourd'hui disjointe de tout ancrage politique.

\section{LES LIMITES DE CE TRAVAIL}

Il ne s'agit pas, ici, d'écrire l'histoire des bibliothèques publiques françaises ni celle des bibliothèques publiques américaines. Les principaux éléments chronologiques et statistiques figurent ici dans les " préambules ». Il s'agit, plutôt, de faire une analyse comparée de la situation de ces bibliothèques : leurs objectifs, les valeurs qui les animent, leurs ambitions, les questions qui se posent à elles, les obstacles, leurs appuis, leurs soutiens. Dans les discours et dans les faits.

Les bibliothèques concernées par ce travail sont, en France, les bibliothèques municipales ${ }^{19}$ et aux États-Unis les Free Public Libraries, qu'elles dépendent des communes ou des comtés ${ }^{20}$. Il n'y a pas de définition officielle de ce qu'est une bibliothèque municipale en France. Aux ÉtatsUnis, une Public Library est définie comme une collection, du personnel rémunéré, une ouverture au public, des ressources nécessaires dont tout ou partie provient de budgets publics ${ }^{21}$.

La période étudiée est donc double : la période des origines, d'une part ; celle qui suit la seconde guerre mondiale, d'autre part. Cette dernière offre une cohérence aussi bien côté français, avec les prémices puis

19. Les bibliothèques des zones rurales, appartenant au réseau d'une BDP, ne sont pas concernées, leur contexte politico-administratif étant trop éloigné de celui des bibliothèques urbaines. L'émergence de l'intercommunalité, encore très récente, n’est pas ici prise en compte.

20. Comme en France, les Rural Libraries, souvent très petites, ne seront pas traitées, non plus que les State Libraries qui sont des agences de développement.

21. National Center for Education Statistics (NCES) : < http://nces.ed.gov/surveys/libraries/ > 
la phase de développement déjà évoquée ; que côté américain où, après la Dépression et la Guerre, les Public Libraries ${ }^{22}$ reprennent le cours normal, « sans histoire », de leur histoire.

Renoncer à un récit chronologique présente des avantages, en particulier celui d'autoriser l'analyse comparée. L'inconvénient majeur, bien entendu, est celui d'écraser l'épaisseur historique. De faire des raccourcis qui semblent confondre la situation de 1950 et celle de 1990. De gommer le contexte, politique, social, culturel. D'enfiler les anachronismes et l'interdire toute compréhension des évolutions. De renoncer à faire de l'histoire.

Deux arguments pour ma défense. Le premier, que cette période d'environ un demi-siècle est assez courte pour que l'analyser d'un seul tenant ne soit pas la dénaturer. Le deuxième, qu'une bonne part de ce travail est basée, au contraire, sur l'analyse des origines, des fondations, des héritages. De la pré-histoire... C'est bien, j'en suis persuadée, l'épaisseur historique d'un siècle et demi, l'épaisseur de ce socle fondateur, qui éclaire l'évolution du deuxième $\mathrm{xx}^{e}$ siècle. Le rapport des bibliothèques et des bibliothécaires à la démocratie, aux pouvoirs, à la politique, à l'éducation, au territoire, à la population est né dans le XIXe siècle américain.

Je ne connais de précurseur à mon travail que celui de Jean Hassenforder, qui date de $1967^{23}$. Il avait, à l'époque, considéré, lui aussi, que l'analyse comparative pouvait, devait éclairer les différences de développement entre bibliothèques. Mais la période qu'il étudie se limite à un grand $\mathrm{XIX}^{\mathrm{e}}$ siècle. Mon propos est davantage contemporain. Cependant, même si aucune étude comparative n'a encore été menée sur les bibliothèques contemporaines, le recours à la comparaison avec l'étranger est un exercice de style fréquent chez les bibliothécaires : le " retard français » est une figure rhétorique très usitée depuis Eugène Morel. Et, très souvent, ce " retard » est mesuré à l'aune du développement des bibliothèques américaines ${ }^{24}$. "Le temps est venu, après un demi-siècle d'efforts qui triomphent aujourd'hui en Angleterre, en Amérique, de concevoir la lecture comme un service public, municipal, analogue à la voirie, aux

22. Dans la suite de cet ouvrage, les bibliothèques publiques américaines, au sens entendu plus haut, seront désignées ainsi.

23. Jean Hassenforder. Développement comparé des bibliothèques publiques en France, en GrandeBretagne et aux États-Unis dans la seconde moitié du XIX siècle (1850-1914). Cercle de la Librairie, 1967.

24. Les pays qui servent de buttes-témoins sont les pays anglo-saxons, qu'ils soient d’Europe ou d'Amérique (Anne-Marie Bertrand. "L'éternel retard ». Regards sur un demi-siècle : cinquantenaire du Bulletin des bibliothèques de France, $B B F$, 2006). 
hôpitaux, à la lumière ${ }^{25}$ ", écrit Eugène Morel en 1908. Lui fait écho Michel Melot, 90 ans plus tard : " On peut regretter que la France ait une des rares bibliothèques nationales au monde dont l'entrée soit payante ou que les bibliothèques publiques y soient généralement fermées le soir ou le dimanche, à l'heure où elles pourraient jouer pleinement leur rôle culturel, professionnel ou pédagogique. Les exemples américains ou scandinaves restent encore, sur tous ces chapitres, des points de mire. ${ }^{26} »$ Mais la rhétorique en reste au niveau du constat et la recherche comparative reste à mener.

Les principales limites de ce travail, bien entendu, sont celles de l'auteur, tant il est illusoire d'espérer embrasser tous les éléments permettant de comparer des institutions si différentes dans leur déploiement, les unes à la pointe du développement, les autres, longtemps engluées dans un retard qui semble congénital, et permettant de prendre en compte tous les facteurs d'influence, qu'ils soient politiques, sociaux, culturels, économiques, voire géographiques. Tentative illusoire que j'assume et que je vais essayer de dépasser en me concentrant sur les fondements, les objectifs, les valeurs qui portent ces établissements.

Entre proximités et différences, similitudes et contrastes, je vais donc tenter d'explorer l'histoire culturelle et l'histoire politique des bibliothèques publiques de nos deux pays. Entreprise sans doute nécessaire mais hardie - et les propos de Jerome Charyn me laissent humble et peu optimiste : "Je n'ai jamais entendu un Français m'apprendre quelque chose sur l'Amérique, ni un Américain quelque chose de sensé sur la France. Les deux cultures ne se comprennent pas. ${ }^{27}$ "

Et Philip Roth ne me rassure pas : " Peut-être qu'avec Pastorale américaine le lectorat français a eu pour la première fois le sentiment qu'on lui livrait un peu les arcanes de l'Amérique et qu'avec les romans suivants, dont La Tache, les gens se sont dits : effectivement, là, j'ai appris quelque chose. Je crois que cet écrivain me dévoile une part de vérité, et qu'il instruit un peu de mon ignorance de l'Amérique. Car il faut bien dire que nous sommes ignorants de nos pays respectifs. D'une ignorance inouïe. ${ }^{28}$ »

\footnotetext{
25. Eugène Morel. Bibliothèques. Mercure de France, 1908.

26. Préface à Les bibliothèques en France, 1991-1997, sous la dir. de Dominique Arot, Éditions du Cercle de la Librairie, 1998, p. 15.

27. Le Monde 2, 2 octobre 2004.

28. Interview sous le titre « L’amateur confirmé ». Le Monde 2, 11 septembre 2004.
} 


\section{Quelques problèmes de traduction}

\section{Public Library}

La Public Library américaine ne peut être traduite ni par bibliothèque municipale puisqu'elle englobe aussi des bibliothèques relevant des comtés et que, dans les communes, ce ne sont pas exactement des services municipaux; ni par bibliothèque publique, que je réserve, ici, au modèle français de bibliothèque lentement conçu au fil du $x^{e}$ siècle et lentement mis en œuvre à partir des années 1960. J'ai renoncé à traduire par « bibliothèque à colonnade grecque », comme m'y invitait cependant Robert Leigh : "Il n'est pas difficile d'identifier la Public Library dans une commune moyenne, avec ses colonnes grecques et son porche [...]. Mais la Public Library Inquiry n'avait pas les moyens d'étudier tous les types de bibliothèque. Aussi, nous nous sommes limités à l'institution publique soutenue par l'impôt, avec ses colonnes grecques ${ }^{29}$. »

Je conserverai donc, sans la traduire, l'appellation de Public Library.

\section{Library Board}

(ou Board of Trustees)

On pourrait traduire par Conseil d'administration, qui serait une bonne manière de marquer à la fois le caractère de gouvernance privée (comme dans une entreprise) et le caractère de participation à une activité collective (comme dans une association). Mais ce serait trop méconnaître le caractère singulier de cette institution, typiquement anglo-saxonne.

Je conserverai donc, sans la traduire, l'appellation de Library Board.

\section{Community}

Voilà bien un mot significatif des différences entre la société française et la société américaine (d'ailleurs, pour marquer leur opposition à « l'américanisation » de la société française, ses critiques brocardent volontiers le « communautarisme » américain).

Il se trouve que les Américains, eux-mêmes, semblent perplexes sur l'emploi de ce mot et sur ses sens différents en fonction des époques et des contextes - communauté universitaire, groupes professionnels, population d'une ville ou d'un quartier, groupes religieux, ethniques, à préférence sexuelle, etc.

Je le traduirai donc en fonction du contexte dans lequel il est employé.

29. Robert D. Leigh. The Public Library in the United States: The General Report of the Public Library Inquiry. New York, Columbia University Press, 1950. Je reviens dans le chapitre I sur la Public Library Inquiry. 


\section{Intellectual Freedom}

«Intellectual Freedom » ne signifie pas exactement liberté d'information, mais ce me semble être la moins mauvaise traduction puisqu'il s'agit, ici, d'avoir accès à l'information, sans censure ni obstacles. "Liberté intellectuelle », en français, a une signification davantage tournée vers la production d'information que vers sa réception. La définition qu'en donne David Berninghausen est " pour tous les usagers des bibliothèques, un accès libre à l'expression de tous les points de vue, sur tous les sujets qui prêtent à discussion » (David Berninghausen, The Flight for Reason: Essays on Intellectual Freedom, ALA, 1975).

\section{Encore une précision}

Par convention (dont j'assume le caractère jacobin), l'État central ou fédéral sera écrit avec une majuscule et les états, qui forment ensemble les United States, avec une minuscule. 


\section{PRÉAMBULES}

\section{PRÉAMBULE 1 : BREF RÉCIT}

L'

9 histoire des bibliothèques municipales en France depuis 1945 peut être écrite comme une lente avancée, une croissance, une marche qui s'accélère. Un mouvement presque immobile, puis lent, puis rapide. Ce tempo général est lié, évidemment, au mouvement de la société mais d'une liaison décalée, différée. Toujours un peu en retard, un peu à côté. Tentons un bref récit de ce demi-siècle ${ }^{30}$.

\section{Largo}

Après la guerre, la situation des bibliothèques municipales est doublement problématique : à l'inertie des décennies précédentes s'ajoutent les effets du conflit.

En 1944, l'inspecteur général des bibliothèques, Pierre Lelièvre, décrit ainsi l'état de ces bibliothèques : "Dans la plupart des grandes villes, la Bibliothèque Municipale est essentiellement une bibliothèque de conservation (manuscrits, livres anciens, reliures de prix, éditions rares), d'étude et de documentation, fréquentée par les érudits locaux, le corps enseignant, les étudiants et les élèves de l'enseignement secondaire ou supérieur. La lecture populaire ne s'y trouve qu'en parente pauvre ou déshéritée. Dans beaucoup de petites villes, où le public studieux se réduit à quelques retraités, la Bibliothèque Municipale tend vers le cabinet de lecture, mais bien rarement réalise ce type de bibliothèque accueillante et vivante qui seule peut gagner au livre de nouveaux lecteurs [...]. Est-ce à dire qu'il n'existe point de bibliothèques de prêt ? Tout au contraire, l'abstention de l'État et la nonchalance des municipalités ont favorisé l'éclosion et la multiplication des bibliothèques semi-publiques : cercles, ligues, associations, patronages, sociétés littéraires ou savantes, syndicats profession-

30. Ce récit est inspiré de mes travaux, Les bibliothèques municipales : enjeux culturels, sociaux, politiques, Éditions du Cercle de la Librairie, 2002 et Les villes et leurs bibliothèques : légitimer et décider (1945-1985), Éditions du Cercle de la Librairie, 1999, auxquels on pourra se reporter pour une analyse développée. 
nels ou "amicales" ont très souvent de petites collections d'ouvrages qu'ils prêtent à leurs membres. Mais la bonne volonté ne compense point l'incompétence, et aux inconvénients d'une mauvaise gestion technique, s'ajoutent les difficultés financières. »

En 1948, la jeune Direction des bibliothèques et de la lecture publique dresse à son tour un panorama de la situation à la Libération : « En dépit des nombreux projets et rapports qui depuis 1930 avaient été proposés, en dépit de quelques expériences qui donnaient la voie à suivre, la situation dans laquelle se trouvait la France au début de 1945 en matière de lecture publique pouvait surprendre bien des étrangers et, disons-le sans hésiter, consternait également tous les bibliothécaires français impatients de rattraper le temps perdu, mais toujours paralysés à un moment donné dans leurs entreprises par le manque de crédits, et déplorant l'absence d'un organisme central pour les appuyer ou les aider. ${ }^{31}$ "

Désintérêt, consternation, nonchalance, incompétence : tel est le contexte dans lequel vivotent les bibliothèques municipales en France. On compte alors environ 400 bibliothèques municipales - comptées par un outillage statistique bien rudimentaire. Parmi ces quelques centaines d'établissements, on estime à une soixantaine le nombre des bibliothèques " sinistrées " soit dans la campagne de 1940 soit dans les bombardements des années 1943-1944, dont 29 ont été complètement détruites, bâtiments et collections confondus, les plus importantes étant celles de Brest, Caen, Chartres, Dieppe, Douai, Saint-Nazaire ou Tours.

De cette situation léthargique, les administrations et les bibliothécaires sont conscients. Pour y remédier, ils comptent sur l'État, sommé d'agir par le biais d'une administration centrale, d'une organisation du territoire et d'une loi sur les bibliothèques. Ce triptyque, on le sait, a innervé toute la période étudiée ici.

Le regard, l'analyse des contemporains, cependant, sont sans complaisance. La situation est alors décrite et vécue comme catastrophique mais pleine d'espérance. Constante, là aussi, de la rhétorique : « Un schéma discursif durable est ainsi mis en place : dire l'importance décisive de la lecture publique dans une nation démocratique et éclairée ; reconnaître l'état insatisfaisant de la situation actuelle (héritage du passé, conséquence de la crise, ou de la guerre, ou des faiblesses du gouvernement précédent) ; annoncer que cela ne saurait durer ; relever les signes qu'on 
a d'espérer [...]. Le temps passe : dix ans, trente ans, cinquante ans plus tard, on retrouve le même discours, pétri de la même farine. ${ }^{32}$ "

Pendant les décennies 1950 et 1960, c'est bien ce schéma qui est à l'œuvre : on glose sur ce qu'il faut faire (une loi, une organisation du territoire) mais on fait bien peu. Les villes sont hors d'état (politique et budgétaire) d'agir, l'État s'intéresse d'abord à ses bibliothèques universitaires. La lecture publique est soutenue par le seul biais des bibliothèques centrales de prêt, organismes créés en 1945 pour desservir les zones rurales à un moment où l'exode rural s'accélère.

Mouvement immobile...

\section{Adagio}

Le milieu des années 1960 marque le début de l'accélération, grâce à la conjoncture entre l'évolution de la société et la volonté politique du premier ministre, Georges Pompidou. Ce frémissement aurait pu, une fois de plus, rester sans lendemains mais, à partir des années 1970, les villes prennent le relais et le mouvement, dès lors, est lancé.

On a déjà beaucoup glosé sur le « Tout est à faire » en matière de lecture publique, formule de Georges Pompidou, normalien adepte de la diffusion du livre et de la lecture. Le comité interministériel mis en place à la fin 1966, aiguillonné par les bibliothécaires modernistes, formalise une nouvelle doctrine de la bibliothèque publique qui prend en compte les bouleversements sociaux et urbains de la société : l'augmentation de la population scolarisée, les grands ensembles, les maisons de jeunes sont évoqués comme arguments pour une nécessaire modernisation des bibliothèques. Les préconisations techniques s'inspirent clairement du modèle anglo-saxon, avec le libre accès aux rayons, la création de collections de disques, la création de bibliothèques dans les quartiers et, partout, des sections pour les enfants.

C'est que, sur ce point en particulier, la situation continue à être désastreuse : en 1964, une enquête ne recense que 49 bibliothèques pour enfants, auxquelles il faut ajouter 7 " coins pour les jeunes ». Les enfants sont généralement accueillis à partir de 7 ans. Faute de personnel, seules 22 de ces bibliothèques peuvent ouvrir tous les jours ${ }^{33}$. La création de la Joie

32. Anne-Marie Chartier, Jean Hébrard. Discours sur la lecture (1880-1980). BPI, 1989, p. 78-79.

33. Marcelle Bouyssi. «Les sections pour la jeunesse dans les bibliothèques municipales de province ». Bulletin des bibliothèques de France, t. 9, n 7, 1964. 
par les livres, en 1965, grâce à un mécénat hérité du modèle américain, est un ferment décisif pour montrer ce que peut être une bibliothèque enfantine et pour former les bibliothécaires français qui, à l'époque, étaient encore nombreux à se demander si l'accès direct aux livres en rayons n'était pas un encouragement au vol des livres...

La postérité du comité interministériel de 1966-1967, dont le rapport est publié amputé du volet financier ${ }^{34}$, sera surtout incitative. Non seulement, le taux de subvention accordé par l'État peut désormais monter jusqu'à $50 \%$ des dépenses mais encore tout un outillage pédagogique est créé et mis en place par l'administration centrale : des statistiques, des normes, des réunions avec les élus au cours desquelles le discours modernisateur passe peu à peu. D’autant plus que les moyens des villes, qui sont les opérateurs et les décideurs, progressent sensiblement dans la décennie 1970 : le budget des collectivités locales augmente de $80 \%$ de 1970 à 1985, l'effectif du personnel communal de 331000 agents en 1962, à 590000 en 1977 et 833000 en $1983{ }^{35}$. Et ce personnel est de plus en plus qualifié : le nombre des diplômés de l'enseignement supérieur parmi le personnel municipal s'accroît de 144 \% entre 1974 et 1986. Les villes, dotées d'un véritable pouvoir d'expertise, peuvent désormais mener de véritables politiques municipales, et donc des politiques culturelles.

Les années 1970 voient ainsi la conjonction entre une société de plus en plus urbanisée et scolarisée, un modèle de bibliothèque diffusé par l'appareil d'État, des opérateurs (les villes) aptes à prendre en charge le développement de ces établissements. Et le mouvement s'accélère : 1972, une très grande bibliothèque s'ouvre à Lyon ; Caen (1972), Montreuil (1974), Argenteuil (1975), Cambrai (1975) suivent... La décennie 1970 aura construit huit fois plus que la décennie précédente ${ }^{36}$. La Bibliothèque publique d'information, ouverte en 1977, est le symbole de ce mouvement modernisateur.

\section{Allegro}

Le mouvement de modernisation s'accélère. Non seulement les moyens des villes augmentent, mais encore les établissements ouverts dans les années 1970 rencontrent le succès : argument très fort auprès des élus. Le

34. La lecture publique en France. La Documentation française, 1968.

35. Dominique Lorrain. "La montée en puissance des villes ». Économie et humanisme, $\mathrm{n}^{\circ}$ 305, 1989.

36. $41000 \mathrm{~m}^{2}$ dans la décennie 1960, $324000 \mathrm{~m}^{2}$ dans la décennie 1970. 
nombre des inscrits double en dix ans (entre 1977 et 1987). Cet argument, au départ employé par les inspecteurs généraux et les bibliothécaires pour emporter la conviction des élus, est désormais intégré par les élus eux-mêmes : leurs efforts en faveur des bibliothèques sont légitimés par la réponse qu'y apporte la population. Ainsi, Jean-Pierre Sueur, maire d’Orléans, répondant à son challenger aux élections municipales de 1995 qui l'accusait de mener une politique élitiste : « La médiathèque a reçu 250000 personnes depuis son ouverture, soit quatre fois plus que l'ancienne bibliothèque rue Dupanloup. ${ }^{37}$ "

C'est pourquoi les interrogations du début du $\mathrm{XxI}^{\mathrm{e}}$ siècle sur la pérennité de ce succès ont des échos importants. Le nombre des fréquentants reste encore aujourd'hui, alors même que les services à distance se multiplient, l'aune à laquelle on mesure le succès de l'établissement et la légitimité des efforts qui sont consentis pour lui.

Trente ans de développement

\begin{tabular}{|l|r|r|r|r|r|r|r|r|r|}
\hline & 1971 & 1977 & 1980 & 1983 & 1987 & 1991 & 1995 & 1999 & 2004 \\
\hline Nombre de BM & 715 & 948 & 930 & 1106 & 1366 & 1885 & 2315 & 2795 & 2913 \\
\hline Emplois & 3062 & 5815 & 7169 & 9590 & 11632 & 13330 & 16374 & 19315 & 21500 \\
\hline Surfaces ${\left(m^{2}\right)}^{*}$ & 355 & 598 & 679 & 825 & 1093 & 1308 & 1634 & 1852 & 2300 \\
\hline Inscrits * & 1182 & 2251 & 2609 & 3394 & 4236 & 5812 & 6250 & 6582 & 6740 \\
\hline
\end{tabular}

* en milliers

Source : Direction du livre et de la lecture.

Les progrès les plus visibles sont, évidemment, ceux qui concernent les bâtiments. Les aides de l'État continuent à avoir un effet démultiplicateur. Les surfaces des locaux dépassent désormais ceux que, par exemple, le rapport Pingaud-Barreau en 1981 estimait nécessaire d'atteindre (1 million de $\mathrm{m}^{2}$ ) comme ceux que le rapport Vandevoorde préconisait (2 millions de $\left.\mathrm{m}^{2}\right)^{38}$. Après une période (les années 1970 et 1980) où la modestie architecturale avait pour ambition de fondre la bibliothèque dans le tissu urbain pour la fondre dans le tissu social, depuis la fin des années 1980 on a vu apparaître une autre logique, logique d'image et d'affirmation.

38. Bernard Pingaud, Jean-Claude Barreau. Pour une politique nouvelle du livre et de la lecture. Dalloz, 1982 : « Passer de $600000 \mathrm{~m}^{2}$ à 1 million »; Les bibliothèques en France. Dalloz, 1982 : « Il serait indispensable de construire ou reconstruire environ $1400000 \mathrm{~m}^{2}$ de nouveaux bâtiments ». 
Désormais, dans les villes où la concurrence est rude pour faire venir des emplois qualifiés, il faut mettre la qualité de vie dans la balance (un arrêt TGV, des classes préparatoires, une politique culturelle attractive) : la construction de bâtiments prestigieux, confiée à de grands noms de l'architecture, participe de ce « négoce de la notoriété » (Emmanuel Wallon). Les bibliothèques en bénéficient elles aussi.

Cette importance accordée à la qualité architecturale est un des éléments de la « médiathèque » qui fleurit aujourd'hui en France, et qui fait « sortir la bibliothèque de son traditionnel quadrilatère, morceau de sucre ou boîte à chaussures, et lui donne des formes surprenantes, qui sont l'expression de sa diversité, de son ouverture et un signal dans la ville. ${ }^{39}$ " Médiathèque, mot intraduisible, surinvesti par les élus pour qui il est synonyme de modernité ${ }^{40}$. Modernité symbolique, modernité technique. Les bibliothèques municipales sont aujourd'hui envahies par les écrans, proposent des revues électroniques, des DVD, l'accès à Internet...

« D’ici une décennie, la bibliothèque ne sera plus, pour le chercheur, qu'une borne de consultation publique, pratique et facilement accessible de n'importe où, même de chez lui. Dans l'avenir des bibliothèques, les livres ne sont plus support de la culture, mais objets d'encombrement, de gêne », écrivait-on dans le Magazine littéraire, en octobre 1989. Aujourd'hui, on continue à construire des bibliothèques (plus de 200 chantiers par an). Mais elles ont à se confronter avec le nouveau continent qu'est la documentation numérique, à prouver leur légitimité à une société dont l'imaginaire est séduit, happé par le « tout est accessible » d’Internet. L'offre privée d'information et de culture (presse, musique, films...) remet en question l'offre publique que proposent les bibliothèques.

Autre contrepied : au moment où le dispositif d'incitation étatique connaissait sa plus grande productivité ${ }^{41}$, la décentralisation y mettait fin.

39. Michel Melot, «Le temps des médiathèques ", Regards sur un demi-siècle : cinquantenaire du Bulletin des bibliothèques de France, $B B F, 2006$, p. 226. Michel Melot souligne que le concept de médiathèque n'est, au fond, que l'adoption en France de pratiques bibliothéconomiques, « bien connues des Public Libraries anglo-saxonnes, comme le libre accès, l'information et la documentation, le lieu civique ou convivial de rencontres et d'animations, bref tout ce qui peut la distinguer de la vieille bibliothèque savante réservée aux notables et aux érudits, dont elle a servi à faire le procès et à dépasser le modèle. Voilà pourquoi sans doute, le mot médiathèque désigne un phénomène français, utilisé désormais dans d'autres pays latins, mais intraduisible chez les Américains, où la question d'un aggiornamento ne se pose pas. » (Ibid., p. 208-209)

40. Ainsi, le conseil municipal de Metz décide de baptiser son nouveau bâtiment " médiathèque », terme « qui a l'avantage de supprimer l'aura poussiéreuse, l'image de marque désuète et peu flatteuse qui s'attache au mot "Bibliothèque" dans bien des milieux » (délibération du Conseil municipal, 18 octobre 1977).

41. Au début des années 1980, où les projets des villes pouvaient être soutenus par un bon budget du ministère de la Culture. 
À compter de 1983, la globalisation prévue des aides de l'État, c'est-à-dire la fin des crédits fléchés, est considérée comme un danger redoutable pour le développement des bibliothèques qui auraient alors été soumises (abandonnées, disait-on alors) à la seule bienveillance des villes. La liberté d'action, disait-on aussi, entraîne la liberté d'inaction. Perspective qui mobilise au ministère comme dans le monde professionnel des bibliothèques. Ainsi, la Direction du livre et de la lecture appelle à un moratoire de la fin des aides, voire à leur maintien : « Le retard historique de la France dans le domaine des bibliothèques publiques rend particulièrement aiguë l'échéance du transfert des compétences, en 1986 [...]. En ce qui concerne les bibliothèques municipales, l'effort de développement lancé en 1982 doit absolument être poursuivi et amplifié pour assurer enfin une dynamique irréversible. Les subventions, notamment en équipement, ont un effet très incitatif auprès des communes [...]. Pour assurer un développement raisonné et irréversible des bibliothèques publiques, il me paraît crucial que l'État puisse encore pendant quelques années assurer le développement des services les plus fragiles $\left(\mathrm{BCP}^{42}\right)$ et assister par des subventions la prise de conscience des collectivités locales en faveur de la lecture publique. ${ }^{43}$ "

Ce plaidoyer sera entendu, et une mesure dérogatoire sera instituée sous l'appellation « Concours particulier des bibliothèques municipales »: l'action incitative de l'État demeure - elle est simplement déconcentrée dans les Directions régionales des affaires culturelles.

Pour l'analyse du contexte politique, ce dispositif a une importance qui dépasse la technique budgétaire : désarmée par cette déconcentration, la Direction du livre et de la lecture semble hésiter sur sa place et sur son rôle. La prégnance de la logique de décentralisation annihile toute velléité d'aménagement du territoire - on l'a vu dans le programme " bibliothèques municipales à vocation régionale », utilisé pour augmenter les enveloppes budgétaires et non pour créer un réseau structurant et cohérent. L'une des grandes différences avec les autres pays de niveau comparable de développement est ainsi la non-organisation du réseau de bibliothèques : une multiplicité d'opérateurs (plus de 3000 bibliothèques municipales aujourd'hui), aucune articulation entre elles, pas de services communs. La seule organisation structurante est celle des bibliothèques départementales, chargées de desservir (autrefois) et organiser (aujourd'hui)

42. Pour les sigles, se reporter à la liste en fin d'ouvrage.

43. « Mise à niveau des bibliothèques municipales et centrales de prêt avant l'entrée en vigueur de la loi de transfert des compétences », note du Directeur du livre au Directeur de cabinet, 31 mai 1983. 
les zones rurales et peu peuplées. Les bibliothèques forment un archipel, non un réseau. Chacune est donc restreinte à l'étendue de ses propres moyens et ne bénéficie pas de la richesse collective.

\section{PRÉAMBULE 2 : A SHORT STORY}

L'histoire des Public Libraries aux États-Unis depuis 1945 peut être écrite comme une symphonie, tantôt harmonieuse, tantôt dissonante - mais puissante, vigoureuse.

Contrairement à l'histoire française, c'est, en effet, une avancée collective que voit cette période.

\section{Le constat de départ}

À la fin des années 1940, l'American Library Association (ALA) commandite une grande enquête, financée par la Carnegie Corporation et réalisée par le Social Science Research Council sous la direction de Robert Leigh : la Public Library Inquiry ${ }^{44}$. Grâce à elle, nous avons une photographie précise de la situation des bibliothèques publiques.

On compte alors 7400 Public Libraries, qui desservent 100 millions d'habitants et réalisent 350 millions de prêts de livres par an. Environ 40000 personnes y sont employées, dont 15000 ont une formation professionnelle.

Si les établissements sont nombreux, le service rendu est considéré comme de faible qualité. Paradoxe par rapport à la vocation éducative de la bibliothèque, ce sont les livres de fiction qui sont surtout empruntés ; le service de prêt est beaucoup plus développé que le service de référence, qui reste sous-utilisé. Il y a là, analysent les enquêteurs, un problème de moyens et d'organisation : $65 \%$ des 7400 Public Libraries alors recensées se trouvent dans des communes de moins de 5000 habitants. Cette dispersion n'empêche pas que le tiers de la population (48 millions d'habitants) ne soit pas desservi du tout. Au niveau national, seulement $18 \%$ de la population adulte fréquentent une Public Library.

44. Dix-huit enquêtes thématiques ont été menées. La synthèse est publiée par Robert D. Leigh, The Public Library in the United States: The General Report of the Public Library Inquiry, Columbia University Press, 1950. Il est notable que cette enquête a été lancée par l'association des bibliothécaires (l'ALA), alors que, à la même époque, c'est l'État qui réalisait une enquête en France (La lecture publique en France. La Documentation française, 1948). 
Pour y remédier, Robert Leigh préconise une meilleure organisation des bibliothèques : il faut des unités plus grandes, " pour réduire les inégalités de services nées de l'inégalité des ressources de l'impôt » et constituées en réseau pour que chaque usager ait accès " aux ressources des bibliothèques de tout le pays ». Proposition qu'un pays jacobin ne renierait pas... ${ }^{45}$

Les analystes de la Public Library Inquiry soulignent aussi le peu de soutien que les bibliothèques ont su susciter. " Les bibliothécaires ont échoué, à travers tout le pays, à faire partager aux enseignants d'Amérique l'idée que la bibliothèque fait partie intégrante du système éducatif [...]. Bien que la bibliothèque n'ait pas d'ennemis naturels, elle souffre de ne pas avoir d'alliés politiques naturels [...]. Le domaine des bibliothèques est un secteur sous-doté géré par un personnel sous-payé. Ce n'est pas là l'effet de l'hostilité publique ; aucune bibliothèque dans notre étude n'a à affronter une opposition active à ce qu'elle fait. Personne ne s'oppose à la bibliothèque ; presque tout le monde l'approuve. Mais, avec une égale unanimité, personne ne veut payer beaucoup pour elle. Ce contre quoi les bibliothécaires ont à lutter, c'est l'apathie. ${ }^{46}$ "

Pour que les bibliothèques, au-delà des discours, tiennent toute leur place, il convient de les organiser en réseau et de leur trouver des alliés. L'ALA va s'y employer.

\section{L'organisation du réseau}

Dès 1934, l'ALA adopte le National Plan for Libraries. En 1948, elle publie un nouveau plan pour le développement des bibliothèques publiques, élaboré par deux bibliothécaires chevronnés et estimés : Carleton Joeckel et Lowell Martin ${ }^{47}$. C'est le démarrage d'un long travail pédagogique de construction du réseau. À partir de cette date, les Public Libraries ne se conçoivent plus seules : elles sont, d'une part, un maillon dans le réseau des bibliothèques ; elles sont, d'autre part, intégrées dans leur communauté pour en obtenir le soutien actif.

45. Les bibliothécaires modernistes français ont largement insisté sur la nécessité d'organiser les bibliothèques en réseau. Par exemple, Michel Bouvy : « Pour en sortir, il serait nécessaire de privilégier la notion de réseau, redéfinir les unités de base et prévoir entre elles une collaboration organique » («Examen d'un projet », Médiathèques publiques, n 52, 1980). La lutte pour la bibliothèque de secteur fut un de leurs combats majeurs.

46. Oliver Garceau, The Public Library in the Political Process: A Report of the Public Library Inquiry, Columbia University Press, 1949, p. 115, 135 et 111.

47. Carleton Joeckel, Lowell Martin. National Plan for Public Libraries Service. ALA, 1948. 
Pour organiser le réseau, l'ALA entreprend d'élaborer et diffuser des standards : après les "Post-War Standards for Public Libraries" (1943), citons "Public Library Service, a Guide to Evaluation, with Minimum Standards" (1956) ou "Minimum Standards for Public Library Systems" (1966). Désormais, les standards sont élaborés dans le cadre de " systèmes de bibliothèque ", c'est-à-dire des bibliothèques liées, formellement ou non, pour mettre ensemble leurs collections et leurs services à disposition d'un territoire - par définition, une bibliothèque non intégrée dans un système est considérée comme hors standard. Seules des bibliothèques liées entre elles peuvent apporter un service de qualité à tous. Ce parti clair et clairement affirmé ${ }^{48}$ a probablement contribué au développement massif des Public Libraries pendant les décennies suivantes doublement du nombre des bibliothèques, triplement du personnel, quintuplement des collections.

Ce développement est, par ailleurs, soutenu par les aides fédérales : en 1956 est adopté le Library Services Act (LSA) qui, sous des formes diverses, a perduré jusqu'à nos jours. La philosophie de cette aide est le soutien aux bibliothèques par le développement du réseau : incitation aux regroupements, aide au prêt inter-bibliothèques, développement des State Library Agencies, chargées de coordonner le réseau sur le territoire d'un état. Les aides sont attribuées sous réserve de l'existence d'un plan de développement au niveau de l'état.

Peu à peu, le LSA, devenu LCSA (Library Construction and Services Act), devint une sorte de dispositif fourre-tout destiné à aider le développement des Public Libraries dans les zones rurales, mais aussi les constructions, la mise en réseau des bibliothèques, les services destinés aux handicapés, les services pour les personnes âgées, les services de référence à vocation régionale des grandes bibliothèques métropolitaines, la recherche et le développement des State Library Agencies dont le pouvoir croissant semble bien être un des principaux effets de ces aides.

De soutien aux zones rurales qu'elles étaient, les State Library Agencies sont, en effet, devenues des agences de développement et de planification sur l'ensemble de l'état, y compris pour les grandes bibliothèques. Les plans de développement prennent en compte l'accès aux documents, la desserte de publics spécifiques (handicapés, minorités ethniques) et la

48. Les standards de 1956 disent ainsi : « Les Public Libraries doivent être liées (tied) ensemble en un réseau qui mènera à l'égalité d'accès au service et offrira les ressources des bibliothèques les plus riches à toute la population. » 
formation des personnels. Les grandes bibliothèques, comme les Public Libraries de Chicago, Detroit ou Philadelphie sont aidées parce que et pour autant qu'elles autorisent un accès libéral à leurs collections de référence et de recherche. Au fur et à mesure que le réseau s'organise et se renforce, le prêt entre les bibliothèques augmente : il passe de 5,5 millions de documents en 1974 à 14 millions en 1992 et 36 millions en 2005.

Car cette organisation volontariste permet bel et bien de mettre tous les documents de toutes les bibliothèques à disposition de tous. Les bibliothèques sont vues comme un service à la fois local et national (a natural resource and a nationwide service ${ }^{49}$ ) et doivent être organisées au niveau national - mais pas par l'État fédéral, par les bibliothécaires suivant les programmes de l'ALA. En 1986, Michael Gorman résume ainsi cette organisation en réseau : "Il n’y a plus désormais une bibliothèque, il y a LA bibliothèque - fusion de toutes les bibliothèques grâce à leur coopération. ${ }^{50}$ » En 2005, 76 \% des Public Libraries faisaient partie d'un réseau ${ }^{51}$.

\section{Une marche en avant (entravée ?)}

Entre 1948 et 1983, le nombre des Public Libraries est passé de 7400 à 15 500, le personnel de 40000 à 105 000, les collections de 125 à 600 millions de volumes, les usagers de $18 \%$ de la population à $51 \%{ }^{52}$. Dès 1984 , la quasi-totalité de la population est desservie. À l'époque, 48 Public Libraries possédaient plus d'un million de documents ${ }^{53}$.

En 1992, l'ALA souligne fièrement qu'il y a plus de Public Libraries aux États-Unis que de MacDonald's, que $66 \%$ des adultes fréquentent une bibliothèque, que les prêts se sont élevés à 1,3 milliard et que plus d'enfants (700 000) ont participé à un atelier de lecture pendant les vacances d'été qu'à des stages de base-ball ${ }^{54}$.

En 2005, les 9198 Public Libraries ont prêté 2,1 milliards de documents, dont 716 millions aux enfants. $99 \%$ de la population ont un accès

49. Alice Gertzog, Edwin Beckerman. Administration of the Public Library. The Scarecrow Press, 1994, p. 36 (à propos du National Plan for Libraries de 1936).

50. Michael Gorman. "Laying Siege in the Fortress Library”. American Libraries, May 1986.

51. Public Libraries in the United States: Fiscal Year 2005. NCES, 2007.

[En ligne] < http://nces.ed.gov/surveys/libraries >

52. Mary N. Maack. "Public Libraries in Transition: Ideals, Strategies and Research". Libraries \& Culture, Winter 1994.

53. Une statistique similaire n'existe pas en France. À ma connaissance, ce n'est le cas que de deux bibliothèques municipales (Paris et Lyon).

54. "Little League baseball", cité dans Your Right to Know: ALA Library Campaign Book. ALA, 1992, p. 21. 
public à Internet grâce aux Public Libraries. Elles disposent de 138000 emplois (équivalent temps-plein) qui ont répondu à 302 millions de questions des usagers. Le nombre d'entrées dans les Public Libraries était de 1,4 milliard ${ }^{55}$.

La fierté avec laquelle les bibliothécaires parlent de leurs bibliothèques a sans doute deux raisons : la réalité de chiffres impressionnants et la nécessité de conserver l'appui de la population, qui décide (bien plus directement qu'en France) du montant des budgets alloués. D’où l'accent mis sur les services rendus.

En effet, l'adhésion de la population est plus que jamais nécessaire : la marche en avant, majestueuse, des Public Libraries trébuche une première fois pour des raisons économiques dans les années 1960. Les grandes villes, sous l'effet des difficultés budgétaires et des révoltes raciales, se vident des classes aisées au profit des banlieues. Les États-Unis deviennent une " nation suburbaine » (André Kaspi). Les bibliothèques en subissent vite les conséquences : dans la décennie 1960, alors que les prêts augmentent au niveau national de $18 \%$, ils diminuent de $12 \%$ dans ces grandes villes; dans la décennie 1970, les prêts augmentent au niveau national de $9 \%$ et diminuent de $10 \%$ dans les grandes villes. Deuxième faux pas dans les années 1980 sous l'effet de la Tax Revolt. En Californie, l'adoption de la Proposition 13 par référendum en 1978 limite le montant des impôts : dès l'année suivante, le budget d'achat de documents y diminue de $20 \%$ en moyenne et les heures d'ouverture des bibliothèques de $22 \%{ }^{56}$. Cette logique de diminution des impôts fait tache d'huile et on en voit vite les conséquences. Les dépenses d'acquisitions qui avaient augmenté, au niveau national, de 161 millions de dollars à 382 millions entre 1973 et 1978 stagnent entre 1978 et 1983 (de 382 à 388 millions) ${ }^{57}$. Désormais, le combat pour de bons budgets est le fil rouge de cette histoire.

Fil rouge qui est, évidemment, marqué de la singularité de chaque histoire : « La Public Library dans un village ou une ville particulière ne dessert pas le public ; elle dessert sa communauté spécifique, quelle que soit la définition qu'on lui donne. ${ }^{58} »$ Ce combat est donc marqué de suc-

55. Public Libraries in the United States, op. cit. Il est frappant que les statistiques françaises comptent les inscrits en bibliothèque alors que les statistiques américaines comptent les entrées.

56. Lawrence White. The Public Library in the 1980s: The Problems of Choice. Lexington Books, 1983, p. 23-24 et 12. À propos des grandes villes, Lawrence White évoque « la fuite des classes moyennes vers les banlieues, son remplacement par des individus à moindres revenus et moins enclins à fréquenter les bibliothèques. "

57. Bowker Annual 1976, p. 230-231, 1981, p. 274-276, 1985, p. 478-479.

58. Charles R. Mc Clure, John C. Bertot. "Creating a Future for Public Libraries: Diverse Strategies for a Diverse Nation”. Library Trends, Summer 1997. 
cès et d'échecs, en raison de la place que chaque ville, comté, communauté, reconnaît à sa Public Library.

Un signe encourageant est la surface des bâtiments construits ou réhabilités chaque année : il s'est construit ou réaménagé $250000 \mathrm{~m}^{2}$ en 1977, avant une chute sévère en 1978 (184 000) mais la situation s'est rétablie dès 1979 et, bon an mal an, tourne autour des $300000 \mathrm{~m}^{2}$ annuels jusqu'au début des années 1990 et ensuite autour de 400 000, dans environ 200 chantiers annuels. Les budgets consacrés aux bâtiments dépassent les 300 millions de dollars depuis 1991, les 500 millions depuis 1994, les 600 millions depuis $2001^{59}$. Ce flux d'argent étonne même les analystes des statistiques : « Le budget cumulé pour 1988 atteint 243 millions de dollars, en hausse sur les 217 millions de 1987. L'argent est clairement disponible et l'avenir des bibliothèques est brillant, du moins en ce qui concerne les constructions. ${ }^{60}$ » $\mathrm{Ou}$ bien : "Si le public accepte de dépenser plus d'un demi-milliard de dollars pour de nouvelles installations, cela signifie indubitablement qu'il considère les bibliothèques comme essentielles à son bien-être et à son avenir. ${ }^{61}$ »

La place de la bibliothèque dans la société : voilà ce qui importe aux ÉtatsUnis. La place de la bibliothèque dans les programmes gouvernementaux : voilà le souci français. Dès le récit du dernier demi-siècle, l'écart semble considérable. 
CHAPITRE I
VOISINAGES 


\section{CHAPITRE I VOISINAGES}

A vant d'entamer cette entreprise comparatiste, il me semble opportun d'interroger les points de convergence, les connexions, les influences, les curiosités ou, au contraire, les refus, les évitements qu'ont pu entretenir bibliothèques et bibliothécaires de part et d'autre de l'Atlantique. Avant de comparer chacun dans son identité, voir ce qui est à la frontière, au point de contact ou même, simplement, ce qui est connu de l'autre. Ici, je souhaite donc examiner le contexte du voisinage, avant d'analyser, ensuite, tout au long de cet ouvrage, chacune des entités dans sa singularité et ses différences.

Il est peut-être inutile de souligner, en préambule, la disparité de ce voisinage, le déséquilibre qui fait que la culture américaine est largement présente en France, que la littérature américaine est largement présente en France, que le modèle de bibliothèque américain est largement présent en France, mais que l'inverse n'est pas vrai.

Par contre, il n'est peut-être pas inutile de souligner la diversité des situations des bibliothèques américaines et françaises. Deux exemples : en 1980, Jacqueline Gascuel produit, à l'appui d'un article déplorant le retard français, des statistiques redoutables : en 1975, la bibliothèque de Nice, 300000 habitants, a la même activité que celle de Boston, 300000 habitants, en $1875^{62}$. Dans la même veine, la même Jacqueline Gascuel souligne en 1976 que débattre du modèle de bibliothèque n'est plus alors un exercice abstrait puisque des réalisations sont déjà inscrites dans la pierre : «Pour tous ceux qui depuis des années militent en faveur d'une nouvelle conception de la bibliothèque, la pas qui a été franchi est décisif.

62. Jacqueline Gascuel, « Réflexions incongrues à partir de quelques chiffres », Bulletin d'informations de l’ABF, $\mathrm{n}^{\circ} 107,2^{\mathrm{e}}$ tr. 1980.

63. Jacqueline Gascuel, « La Pierre... et la lecture publique », Bulletin d'informations de l'ABF, $n^{\circ} 90$, 1976.

64. Cité par Sidney H. Ditzion, Arsenals of a Democratic Culture, Chicago, ALA, 1947, p. 168.

65. Les films américains représentaient $40 \%$ des entrées en France en 2005 ; le cinéma français $2 \%$ des entrées aux États-Unis - 26 millions sur 1,3 milliard (Le Monde, 16 février 2006). En 2003, 8 des 10 plus gros succès étaient des films américains.

66. Douze romans américains sont classés parmi les 50 meilleures ventes, dont en numéro 1 le Da Vinci Code de Dan Brown ; et aussi Patricia MacDonald, Mary Higgins Clark, Danielle Steel, Mitch Albom, Robin Cook, Erica Spindler, Heather Graham, Gwen Hunter, Steve Hodel, Philip Roth et Matthew Pearl (Livres Hebdo, n 584, 14 janvier 2005). 
Ce n'est plus dans un désert qu'ils ont à évoquer la "bibliothèque publique" comme une oasis inaccessible. Des modèles enfin existent. Une nouvelle génération de bibliothèques municipales est apparue ${ }^{63}{ }$. Cent ans plus tôt, Melvil Dewey écrivait : " Jadis, les bibliothèques étaient peu ouvertes et les visiteurs venaient rarement, comme lorsqu'on vient quelquefois dans un château désert ou une maison hantée. Maintenant, beaucoup de nos bibliothèques sont accessibles aux mêmes heures que les bureaux de poste, et le nombre de nouvelles bibliothèques est si grand que, dans une ville ordinaire, on ne peut plus demander : "Avez-vous une bibliothèque ?" mais : "Où est votre bibliothèque ?", comme l'on demanderait où est votre école, ou votre bureau de poste ou votre église ? ${ }^{64}$ » C'était en 1876.

Il ne peut donc s'agir de comparer ce qui est si inégal. Mais d'analyser deux situations au miroir l'une de l'autre.

Une semaine ordinaire en France (13 au 19 janvier 2005)

Dans la presse, des articles sur le projet de Steven Spielberg de tourner un film sur Abraham Lincoln, le cinquième anniversaire de l'alliance francoaméricaine des musées, des critiques de livres américains (Gentil bébé, Traité de savoir-vivre à l'usage des jeunes Russes), des critiques de films américains (Criminal, The Machinist, Les ex de mon mec), des critiques de disques américains (Bonnie Prince Billy, Paul Westerberg, Home Video, Isis, Xzibit, The Art ensemble of Chicago, Frank Zappa), l'annonce des concerts de Green Day et Hilary Hahn, une critique de l'exposition Shore Stories (Stephen Shore), etc.

$\mathrm{Au}$ théâtre, à Paris, douze pièces d'auteurs américains sont jouées ( $\mathrm{Au}$ nom de l'Amérique, Brooklyn Boy, Chroniques martiennes, Comment devenir une mère juive en 10 leçons, Demande à la poussière, Doux oiseau de jeunesse, J'veux pas être seule(e), Kaddish, Miracle en Alabama, Les Monologues du vagin, Quatre chiens sur un os, Si nous parlions d'amour).

Cinq expositions parisiennes présentent des œuvres américaines (Stephen Shore, Psyke of the wall, Joseph Stieglitz - 2 fois -, Motherwell).

$\mathrm{Au}$ cinéma, trois nouveaux films américains sont projetés à Paris (Les Ex de mon mec de Nick Hurran, Le Fantôme de l'Opéra de Joel Schumacher, Melinda \& Melinda de Woody Allen), 59 autres films américains continuent à y passer et sept réalisateurs ou acteurs font l'objet d'un festival, Cassavetes (2 films), Humphrey Bogart (6 films), Jim Jarmusch (5 films), Ken Russell (4 films), Nicholas Ray (7 films), Woody Allen (6 films), ainsi que le festival Vicente Minnelli à Beaubourg (18 films) ${ }^{65}$.

Dans les librairies, des centaines de titres de livres américains sont disponibles ${ }^{66}$. 


\section{L'INFLUENCE AMÉRICAINE SUR LES BIBLIOTHĖQUES DE FRANCE}

Cette histoire est bien connue - et participe de la saga héroïque des bibliothèques publiques françaises. Mais, nous allons le voir, des débuts prometteurs se sont rapidement enlisés dans l'indifférence du milieu social français.

\section{Tout commence par une solidarité active}

Avant les réalisations philanthropiques de l'après première guerre, le modèle américain s'était déjà invité en France, dès l'Exposition universelle de 1900, où Melvil Dewey et son équipe de la Bibliothèque d'État de New-York présentent une « bibliothèque américaine modèle ${ }^{67}$ ». En 1906, Maurice Pellisson publie Les bibliothèques populaires à l'étranger et en France où il évoque chaleureusement les bibliothèques américaines. En 1908, Eugène Morel publie son célèbre Bibliothèques, essai sur le développement des bibliothèques publiques, où il compare la situation des bibliothèques en France, en Grande-Bretagne et aux États-Unis. En 1913, le président de l'ABF, Charles Sustrac, entreprend un voyage d'étude aux États-Unis.

Ainsi, dès le début du siècle, des bibliothécaires français connaissent et admirent les bibliothèques publiques américaines. Après la première guerre, des bibliothécaires américains sont sur le sol français des « agents du changement qui implantèrent les nouvelles idées et les pratiques déjà connues et soutenues par des pionniers comme Morel ${ }^{68}$. » Pionniers qui œuvrent depuis lors à l'implantation de ces pratiques et de la conception qui les sous-tend ; pionniers qui souffrent, dit même Noë Richter, d'une " anglo-saxomanie endémique ${ }^{69}$ ».

La première manifestation de cet échange culturel est une bibliothèque (qui existe toujours), l'American Library in Paris, fondée en 1920, héritière de la bibliothèque de guerre montée en 1917 par l'American Library Association pour les soldats américains. Mais les Américains ne se contentèrent pas de servir leurs propres concitoyens. C'est à la fin de la première guerre que leur solidarité s'est concrétisée en France. Le Comité américain pour les régions dévastées (CARD) est créé en 1917 à l'instiga-

67. Mary N. Maack. "Americans in France: Cross-Cultural Exchange and the Diffusion of Innovations". Journal of Library History, 1986.

68. Ibid.

69. Noë Richter. La lecture \& ses institutions. Plein Chant, 1989. 
tion d'Ann Morgan, l'héritière de la Banque Morgan : d'abord destiné, durant les hostilités, à venir en aide aux populations civiles du département de l'Aisne, le CARD après la guerre prit part à la reconstruction - notamment en créant quatre bibliothèques dans l'Aisne, d'abord dans des petites villes (Blérancourt, Anizy-le-Château, Vic-sur-Aisne, Coucyle-Château) en 1920, puis une cinquième, plus grande, à Soissons en 1921. En 1922, c'est la première bibliothèque pour enfants de France qui ouvre grâce à lui à Paris, rue Fessart, bibliothèque municipalisée en 1924. Un autre organisme charitable, the Book Committee for a Children's Library, crée en 1924 une autre bibliothèque pour enfants, L'Heure Joyeuse, qui connut une postérité plus éclatante ${ }^{70}$. En 1921, le président de la Fondation Carnegie pose la première pierre de la nouvelle bibliothèque de Reims - qui s'appellera Bibliothèque Carnegie, comme tant de bibliothèques aux États-Unis. De nombreux dons contribuent à reconstituer de petites bibliothèques publiques, comme pour la ville de Liévin, à qui des bibliothécaires de Chicago envoyèrent des livres, du mobilier et de l'argent pour aider à la création d'une bibliothèque pour enfants ${ }^{71}$.

Enfin, nouvel intervenant, l'ALA, avec le soutien financier du CARD, crée en 1923 une école de bibliothécaires à Paris. Car philanthropes et bibliothécaires américains savent bien que cette œuvre ne peut être poursuivie que si des bibliothécaires français, à leur tour, sont capables de prendre en charge et, mieux, de promouvoir le modèle américain (c'est-àdire moderne) de bibliothèque publique. L'école vécut jusqu'en 1929, les Américains la confiant progressivement aux mains des modernistes français d'alors (Eugène Morel, Ernest Coyecque, Gabriel Henriot, Henri Lemaître) qui ne purent la faire vivre plus longtemps - Gabriel Henriot réussit cependant à maintenir un enseignement dans le cadre de la bibliothèque Forney.

« La poursuite de cette œuvre américaine fut significative et influença l'histoire des bibliothèques françaises au Xx $x^{\mathrm{e}}$ siècle ${ }^{72}$ », écrit Martine Poulain.

70. Henri Lemaître souligne le caractère spécifique des bibliothèques pour enfants créées grâce aux Américains : " La bibliothèque pour enfants fut choisie parce qu'elle parut de création vraiment américaine ; du tempérament américain, elle tient en effet son principe puisqu'elle a en vue de contribuer au progrès de l'éducation volontaire ; l'enfant doit y aller de son plein gré, il doit y être libre de choisir ses lectures, il doit s'y sentir chez lui et à son aise, d'où l'adage américain que la bibliothèque doit être more a home than a school. » (« La bibliothèque enfantine de la rue Boutebrie, L'Heure Joyeuse ». Revue des bibliothèques, $\left.\mathrm{n}^{\circ} 1-6,1925\right)$

71. Le maire de Liévin, lors de la pose de la première pierre, en 1925, salue "un don de la Grande Amérique" (Laurent Wiart, "La bibliothèque de Liévin : un don de la Grande Amérique", Eulalie, $\left.\mathrm{n}^{\circ} 3,2002\right)$.

72. Martine Poulain. "Philanthropy, Benefaction, and Libraries in France, 1916-1929”, Libraries \& Culture, Spring 1996. 
Mais cette influence ne fut efficace qu'à long terme, car dans les années 1920 ni les bibliothécaires français ni les pouvoirs publics ne furent capables de prendre le relais. Occasion ratée ? Pourtant, combien les plus convaincus d'entre eux, tel Ernest Coyecque, auraient aimé montrer, seulement montrer, ce qu'était une bibliothèque moderne : "Maintenant, je vais pouvoir dire aux incrédules : "Prenez le train et allez voir à Soissons." Ce sera une démonstration par le fait. ${ }^{73}$ »

$\mathrm{Au}$-delà des réalisations, marginales et éphémères, c'est un modèle de bibliothèque qui est introduit en France et cela, par contre, ne mourra pas - comme Laure Léveillé le souligne: "Mais au moment où les Américains quittent la scène, ils laissent derrière eux des expériences fort marginales. Cependant, si les solutions de continuité paraissent inévitables dans le domaine des réalisations matérielles, l'aide américaine ou plus exactement le modèle américain venu à la rencontre des professionnels français n'en exercent pas moins une durable influence sur les représentations de la bibliothèque en France. ${ }^{74}$ » Cette influence a été entretenue par la formation « américaine » reçue par des militants de la modernisation des bibliothèques publiques. Ainsi, Victorine Vérine, bibliothécaire de Soissons et promotrice du premier bibliobus, avait été formée à l'école américaine de Paris. Cette formation sera un des vecteurs les plus efficaces de l'acculturation des bibliothécaires français au modèle américain.

\section{Le modèle américain}

Ce modèle qui a convaincu dès le début du $\mathrm{xx}^{\mathrm{e}}$ siècle est à la fois un modèle technique (le libre accès aux ouvrages, des bibliothèques pour les enfants, l'introduction de la presse, de la documentation pratique, la gratuité, du personnel qualifié) et un modèle politique : celui des bibliothèques pour toute la population, appuyées et voulues par la population.

Jessie Carson, qui fut l'une des responsables du CARD, décrit avec vigueur l'écart entre la conception américaine des bibliothèques et la réalité qu'elle découvrit en France après la première guerre : « Il ne faut pas croire que le CARD ait introduit en France la bibliothèque publique. Il y en a dans tous les quartiers des grandes villes et dans presque chaque petit village. Mais ces bibliothèques ne sont pas l'équivalent des “public

73. Lors de l'inauguration de la bibliothèque, en 1921. Cité par Laure Léveillé, « Fascinations étrangères et naissance de la lecture publique », Histoire des bibliothèques, Tome IV, sous la dir. de Martine Poulain, Promodis-Éditions du Cercle de la Librairie, 1992. 74. Ibid. 
libraries” que nous connaissons aux États-Unis ; elles ne répondent en aucune manière aux besoins de la communauté, elles sont incomplètes et mal organisées : ce sont pour la plupart des salles petites, mal tenues, pauvrement éclairées, avec des rayons resserrés montant jusqu'au plafond ; le public ne peut faire le choix d'un livre qu'en consultant un mauvais catalogue, sale, déchiré, souvent périmé, qu'on doit feuilleter sur un pupitre de bois, à l'entrée de la salle. D'autre part, l'entrée de la bibliothèque est, d'une façon générale, formellement interdite au public, qui est invité à attendre patiemment derrière une balustrade qu'on lui remette le livre dont il a indiqué le numéro. ${ }^{75}$ " L'ouverture au public se traduisit donc à la fois physiquement par la suppression de la « balustrade » et par l'accueil des enfants, mais aussi par le souci de l'accueil des usagers : il s'agit d'une réelle révolution copernicienne. Ancrés sur les collections, l'intérêt et les compétences des bibliothécaires français se déplacèrent progressivement, tout au long $\mathrm{du} \mathrm{Xx}^{\mathrm{e}}$ siècle, vers le souci des usagers.

Le malentendu qui va rapidement s'instaurer est l'abandon des prémices politiques de ce modèle. On n'en retiendra que les caractéristiques techniques, gages de succès. Et on oubliera le discours de Caroline Griffiths, en 1924, lors de l'inauguration de l'Heure Joyeuse : « La bibliothèque pour enfants est sociale par nature et scientifique par son organisation. Pour réussir, elle doit avoir l'appui des autorités publiques, être guidée par des experts et jouir de la sympathie constante et de l'attention perpétuelle des citoyens patriotes ${ }^{76}$ ». Les « citoyens patriotes », j’y reviendrai, ne sont pas considérés en France comme des acteurs du développement des bibliothèques.

L'un des épisodes les plus manifestes de cette admiration infidèle est la conception de la Bibliothèque publique d'information, à partir de 1965 : toute la panoplie technique du modèle américain, et même au-delà, se retrouve dans ce projet lentement mûri et dont son promoteur, JeanPierre Seguin, reconnaît explicitement l'influence américaine, notamment dans la création d'une bibliothèque pour les enfants ${ }^{77}$. Par contre,

75. Texte de 1922, cité par Noë Richter, Introduction à l'histoire de la lecture publique et à la bibliothéconomie populaire. Bernay, À l'enseigne de la Queue du chat, 1995, p. 208.

76. Citée par Viviane Ezratty, « Les bibliothèques françaises pour la jeunesse : quelques questions toujours d'actualité », Bulletin des bibliothèques de France, t. 44, n 3, 1999.

77. Un voyage d'études aux États-Unis, en 1969, lui permit de visiter 24 bibliothèques américaines (Jean-Pierre Seguin, Comment est née la BPI, BPI, 1987, p. 45-47). Mais l'influence la plus forte fut produite par sa visite de l'Amerika Gedenk Bibliothek, « équipement offert par le peuple des USA à celui de Berlin, en mémoire des souffrances endurées par lui pendant le blocus, et qui témoignait des progrès les plus récents des Américains dans le domaine des bibliothèques publiques », rappelle-t-il : «Ce fut pour moi un choc, un véritable éblouissement. » (Ibid., p. 16). 
politiquement, il émane d'une des institutions étatiques les plus vénérables (et les plus institutionnelles) : la Bibliothèque nationale, dont le « département de lecture publique » fut plus tard appelé bibliothèque des Halles, puis Bibliothèque publique d'information. La plus grande, plus moderne et plus fréquentée bibliothèque publique de France est une pure création de l'État.

Enfin, on peut considérer que, dans les années 1980, le modèle américain a disparu de l'horizon d'attente des bibliothécaires français. L'émergence du modèle français de médiathèque, en effet, non seulement continue à ignorer la substance politique du modèle américain, mais en dépasse les caractéristiques techniques - évidemment datées. L'attention portée à la qualité architecturale, la visée conviviale, l'action culturelle sont des apports purement autochtones au modèle de bibliothèque.

Ce qui continue à motiver l'intérêt des bibliothécaires français pour les bibliothèques américaines, c'est la qualité des services rendus et l'innovation. Ainsi, les bibliothèques américaines ne seraient plus un " Autre » à imiter et atteindre, mais un " Même » un peu en avance - ce que signifie, par exemple, ce préambule à un récit de voyage : "Les bibliothèques américaines ont la réputation d'être belles et riches, dynamiques et accueillantes. On y glane beaucoup d'idées et de savoir-faire bibliothéconomique. L'habitude a été prise par les bibliothécaires français, seuls ou en escouade, de traverser régulièrement l'Atlantique pour découvrir les dernières nouveautés technologiques, avec le sentiment de prendre une longueur d'avance sur la réalité de demain. ${ }^{78}$ "

Les intellectuels français gardent, eux, une image forte et positive des bibliothèques américaines. Demeure aujourd'hui dans les esprits l'image d'une bibliothèque riche de collections et accueillante pour ses lecteurs : c'est le discours, par exemple, d'un Pierre Nora, d'un Robert Fossier ou d'un Jean-Marie Goulemot ${ }^{79}$. Mais il s'agit là bien davantage des bibliothèques des universités que des bibliothèques publiques, qu'ils ont peu ou pas fréquentées à l'exception de la New York Public Library - que l'on peut considérer (dans son implantation centrale de la $42^{\mathrm{e}}$ rue) comme une bibliothèque de recherche plus que comme une bibliothèque publique.

78. Françoise Gaudet, Claudine Liéber. « L’Amérique à votre porte : nouveaux usages, nouveaux services, nouveaux concepts de bâtiments... un avant-goût de notre avenir ? ", Bulletin des bibliothèques de France, t. 47, $\mathrm{n}^{\circ}$ 6, 2002.

79. Voir Pierre Nora (passim), Robert Fossier, « La Bibliothèque de la Sorbonne est en danger », Le Débat, n 70, 1992 ; Jean-Marie Goulemot, L'amour des bibliothèques, Éditions du Seuil, 2006, p. 221244, et notamment la dernière phrase (p. 244) : « Lorsque je déciderai d'effectuer mon ultime voyage aux États-Unis, ce sera pour lire une dernière fois en campus afin d'éprouver un avant-goût du Paradis que j'espère. » 


\section{D'autres modes d'influence}

Si le modèle même de bibliothèque ne joue plus son rôle mobilisateur auprès des bibliothécaires, l'influence américaine continue à s'exercer, mais à d'autres niveaux.

D'abord, la richesse des collections des bibliothèques américaines en fait un partenaire incontournable pour tout projet documentaire exhaustif, voire simplement pour tout projet d'envergure. Ainsi, la Bibliothèque nationale de France $(\mathrm{BnF})$ entretient des « relations bilatérales privilégiées avec de nombreuses bibliothèques sœurs, dont la British Library, la New York Public Library, la Bibliothèque du Congrès [...] ", explique son président, Jean-Noël Jeanneney ${ }^{80}$. C'est avec la Bibliothèque du Congrès que la $\mathrm{BnF}$ a créé en mai 2005 un site interactif bilingue sur la présence française en Amérique du Nord du XVI ${ }^{e}$ au XIX ${ }^{e}$ siècle ${ }^{81}$, site qui présente des corpus de documents numérisés. C'est avec la Queens Borough Public Library (de la New York Public Library) que la BPI a conclu une convention de partenariat en 2001, destinée à monter des projets communs ou des formations.

Ensuite, le nombre, la richesse, l'intérêt des bibliothèques américaines suscitent des envies de voyages de la part des bibliothécaires ou futurs bibliothécaires français. Les bourses Fulbright sont le moyen sans doute aujourd'hui le plus utilisé pour entreprendre un voyage d'étude aux ÉtatsUnis : depuis 1951, des dizaines de bibliothécaires français ont utilisé cette possibilité. Des bibliothécaires y ont aussi voyagé ou séjourné dans d'autres conditions : stages à la New York Public Library, à la Newberry Library de Chicago, à la Public Library de Boston, dans des écoles de bibliothéconomie (Graduate School of Library and Information Science à l'université d'Illinois), etc. Quelques voyages d'études ont été organisés à l'occasion de congrès de l'IFLA en Amérique du Nord (Toronto 1968, Washington 1975). Les bibliothécaires modernistes ont visité (Michel Bouvy) ou travaillé (Geneviève Le Cacheux à Cleveland, Geneviève Patte à Ann Arbor) dans des Public Libraries. Comme l'exprime joliment Michel Melot, " le voyage en Amérique ou dans les Pays scandinaves fut aux bibliothécaires [français] du $\mathrm{Xx}^{\mathrm{e}}$ siècle ce que le voyage en Italie fut aux peintres $\mathrm{du} \mathrm{XVII}^{\mathrm{e}} .{ }^{82}$ »

80. Chroniques de la BnF, $\mathrm{n}^{\circ} 29,2005$.

81. La France en Amérique/France in America, < http://gallica.bnf.fr/FranceAmerique/fr > ou < http://international.loc.gov/intldl/ >

82. Michel Melot. « Le temps des médiathèques », op. cit., p. 214. 
Enfin, le nombre et la qualité des bibliothécaires américains en font des éléments moteurs dans de grandes réalisations internationales. Citons la gestion/révision de la classification décimale Dewey, créée par l'Américain Melvil Dewey en 1876 et constamment mise à jour depuis - c'est cette classification que suivent la plupart des bibliothèques publiques du monde. La classification de la Bibliothèque du Congrès, pour sa part, a été adoptée par de nombreuses bibliothèques d'étude et de recherche.

Le plus grand catalogue collectif de bibliothèques, OCLC, est né dans l'Ohio (Ohio College Library Center) et alimente aujourd'hui des milliers de bibliothèques partout dans le monde. L'attribution des numéros identificateurs des livres, l'International Standard Book Number (ISBN), est l'objet depuis 2004 d'une lutte d'influence où Britanniques et Américains mènent la danse. Les Américains sont pionniers en matière d'accès libre à la documentation électronique - ils ont, par exemple, créé la Public Library of Science (PLoS), gérée par les scientifiques eux-mêmes pour contrer la privatisation de l'accès au savoir à laquelle mène le monopole de quelques consortiums éditoriaux.

Enfin, pour mémoire, citons le gigantesque chantier de numérisation ouvert par Google : le noyau initial des collections, celui de grandes bibliothèques universitaires américaines (Harvard, Michigan, Stanford...), s'est progressivement étendu à des bibliothèques européennes, britanniques, néerlandaises, suisses et maintenant, françaises.

Ce contexte scientifique, technique, normatif et économique fait que l'influence américaine sur les bibliothèques françaises est aujourd'hui moins identifiable mais sans doute plus importante que dans les années 1920. S'il n'y a plus de modèle américain évoqué, il y a un environnement américain obligé.

\section{L'influence française sur les bibliothèques américaines}

La question est logique. La réponse sera courte.

$\mathrm{Au} \mathrm{XIX}^{\mathrm{e}}$ siècle, les bibliothèques municipales françaises, savantes, patrimoniales, ont pu susciter l'intérêt de voyageurs américains. La richesse des collections, surtout, semblait un élément d'admiration ${ }^{83}$. Mais, aujourd'hui, c'est l'héritage anglais que les bibliothécaires américains

83. George Ticknor et Edward Everett, de Boston, Joseph Cosgwell, de New York, par exemple, ont visité des bibliothèques françaises dans leurs voyages européens. "Ils montraient d'un doigt envieux les bibliothèques municipales en Angleterre, en France, en Belgique et en Allemagne. » (cité par Sidney H. Ditzion, Arsenals of a Democratic Culture, op. cit., p. 20) 
évoquent (et, en particulier, le Public Libraries Act de 1850) : « Le Library Movement américain a son origine en Angleterre. ${ }^{84}$ " Héritage à la fois reconnu mais aussi radicalement critiqué, voire dénié : « Le mouvement britannique [des Public Libraries] a rencontré une forte opposition de la part de la classe des propriétaires. Le mouvement américain n'a rencontré que rarement l'opposition des contribuables. Les classes privilégiées en Angleterre craignaient un peuple éduqué. Ceux qui gouvernaient la société américaine le favorisaient. Les humanistes en Angleterre s'exprimaient souvent comme s'il s'agissait d'amener une classe de brutes à la civilisation en créant des bibliothèques. Les promoteurs américains d'une vie meilleure étaient généralement égalitaires et profondément démocrates dans leur approche [...]. Sur le long terme, on peut dire que les Britanniques ont plus appris de nous que nous d'eux. ${ }^{85}$ »

Jean Hassenforder souligne, de son côté, les différences considérables qui séparent d'emblée les bibliothèques publiques américaines des bibliothèques municipales françaises : «Aux États-Unis et en Grande-Bretagne, les bibliothèques municipales dès le départ sont des institutions neuves. Elles ne sont point asservies comme en France à de riches fonds anciens. Elles sont libres d'innover. Le même organisme assure des fonctions diverses selon la variété des besoins du présent. Il n'y a point séparation comme ailleurs entre la fonction documentaire et la fonction de grande diffusion du livre. Par là, la bibliothèque acquiert cette dimension minimum préalable à toute action efficace. ${ }^{86}$ "

Un siècle plus tard, si certains pays considèrent qu'ils ont à apprendre du modèle français de médiathèque, ce ne semble pas être le cas des bibliothécaires américains - ce qui ne signifie pas qu'ils ne soient pas intéressés par les bibliothèques françaises ${ }^{87}$. Mais elles ne les influencent pas.

84. Axel Munthe, American Librarianship from an European Angle: An Attempt at an Evaluation of Politics and Activities, Chicago: ALA,1939. W. Boy Rayward se contente de remarquer l'émergence simultanée de la Public Library en Angleterre et aux États-Unis : « Il est intéressant de noter que la Public Library, dans sa version moderne, gratuite et financée par l'impôt a émergé en même temps aux États-Unis et en Angleterre au milieu du XIX ${ }^{e}$ siècle. Un grand nombre de valeurs semblables sous-tendait cette fondation dans les deux pays, mais il n'est pas évident qu'il y ait eu une grande, voire aucune, influence mutuelle » (W. Boy Rayward, "Librarianship in the New World and the Old: Some Points of Contact”, Library Trends, July 1976)

85. Sidney H. Ditzion, Arsenals of a Democratic Culture, op. cit., p. 2-3.

86. Jean Hassenforder, Développement comparé des bibliothèques publiques en France, en GrandeBretagne et aux États-Unis, op. cit.

87. Un bibliothécaire californien, Jack Kessler, édite depuis 1992 un journal électronique (FYI France newsletter) uniquement consacré aux bibliothèques et au monde documentaire en France : $<$ http://www.fyifrance.com > 


\section{ÉCRITURES CROISÉES}

La connaissance, l'intérêt, la curiosité qu'ont les uns pour les autres bibliothécaires français et américains se lisent dans leurs littératures professionnelles. La quantité comme le thème des articles sont significatifs.

\section{Les bibliothèques américaines dans les revues professionnelles françaises}

Le Bulletin des bibliothèques de France $(B B F)$ rend compte régulièrement de la situation des bibliothèques à l'étranger, notamment en matière de modernisation. De 1956 à 2002, le BBF a publié 285 articles (exposés, analyses, comptes rendus de voyages) sur l'étranger. Parmi eux, 59 ont été consacrés aux bibliothèques des États-Unis, de loin la plus importante proportion. Suivent, dans l'ordre, la Grande-Bretagne avec 34 occurrences, l'Allemagne (les deux Allemagnes) 29, l'URSS (puis la Russie) 22, la Scandinavie 15, le Québec 12, etc. L'intérêt pour les bibliothèques américaines porte surtout sur les grandes bibliothèques d'étude et de recherche (à commencer par la Bibliothèque du Congrès) et sur l'adoption de nouvelles techniques (automatisation, audio-visuel, cédéroms, etc.). Les bibliothèques publiques sont peu souvent traitées : un article sur la Boston Public Library en 1981, un sur la Public Library de San Francisco en 1991, deux sur la New York Public Library (1991 et 2002). Des questions transversales, bien sûr, intéressent les bibliothèques publiques, comme la formation des bibliothécaires aux États-Unis ou les questions de censure $(2002,2004)$. Les comptes rendus de voyages des bourses Fulbright concernent généralement les bibliothèques publiques comme les bibliothèques d'étude (1998, 2002).

Le Bulletin d'informations de l'ABF, lui aussi voire lui davantage, consacre régulièrement des pages aux bibliothèques étrangères. La rubrique « Nouvelles internationales » existe dès 1957. Mais on y trouve de tout, le compte rendu de l'association des bibliothécaires (ouest-)allemands, le cinquantenaire de l'association des conservateurs belges, un " hommage soviétique à Julien Cain ", beaucoup de comptes rendus de voyages d'études (surtout en Allemagne et au Danemark), le développement grâce à l'aide internationale de la lecture publique au Cambodge, la présentation de la bibliothèque du Centre culturel français de Yaoundé ou de Barcelone, etc. Parmi les 103 articles retenus (en ne conservant que les articles sur les bibliothèques, c'est-à-dire en éliminant les comptes rendus des congrès et de toutes sortes de conférences comme la conférence européenne des 
bibliothèques médicales ou le colloque des bibliothèques d'art), 24 concernent les États-Unis, qui réalisent comme dans le $B B F$ le plus gros score, suivis par la Grande-Bretagne (20), l'Allemagne (16), l'Europe de l'Est (11), l'URSS et la Russie (8), l'Afrique (8), etc. Ces 24 articles sont surtout des comptes rendus (de voyages : 1957, 1958, 1963, 1966, 1968, 1975, 1980, 1988, ou de congrès de l'ALA : 1957 et 1998) mais aussi des articles thématiques, principalement sur les bibliothèques d'étude (catalogues collectifs, coopération, fonds spécialisés) et moins sur des thèmes intéressant les bibliothèques publiques : les bibliothèques pour enfants (1957), les services pour handicapés visuels (1988), la censure (1997) ou le multiculturalisme (1997).

Dans le $B B F$, ce sont plutôt des Américains (mais pas seulement) qui écrivent sur les bibliothèques américaines ; dans le Bulletin d'informations de l'ABF, ce sont surtout des Français (mais pas seulement). Comme on le verra plus loin, ces quelques publications, somme toute homéopathiques, n'empêchent pas une ignorance profonde de ce que sont les bibliothèques américaines.

\section{Les bibliothèques françaises dans quelques revues américaines}

Se livrer à un décompte symétrique dans la littérature professionnelle américaine est à peu près impossible : il n'y a pas deux revues à dépouiller, mais des dizaines. Ici, je ne livrerai donc qu'un bref aperçu, établi à partir de trois revues : Journal of Library History, Library Quarterly, Library Trends.

Edward A. Goedeken s'est intéressé aux sujets traités dans le Journal of Library History (devenu Libraries \& Culture en 1996) entre 1966 et $2000{ }^{88}$. Sur les 602 articles recensés, près de la moitié (286) sont consacrés à l'histoire des bibliothèques à l'étranger (« Non US ») dont 160 à l'Europe et 24 à la France, toutes bibliothèques et toutes périodes confondues. Quatre articles concernent les bibliothèques publiques françaises au xx siècle $^{89}$. L'intérêt montré aux bibliothèques françaises y est, on le voit, plutôt

88. Edward A. Goedeken. "What We Wrote About and Who We Were: Historical Writings in JLH/L\&C, 1966-2000”, Libraries \& Culture, Summer 2002.

89. Mary N. Maack. "Americans in France: Cross-Cultural Exchange and the Diffusion of Innovations", Journal of Library History, Spring 1986, p. 315-332.

Mary N. Maack. "Women Librarians in France: The First Generation", Journal of Library History, Fall 1983, p. 407-449.

Martine Poulain. "Philanthropy, Benefaction, and Libraries in France, 1916-1929", Libraries \& Culture, Spring 1996.

Noë Richter. «Public Libraries », Journal of Library History, Winter 1984, p. 47-72. 
mince. Il est pourtant encore plus faible dans les deux autres revues évoquées.

La revue Library Trends ne publie que des numéros entièrement thématiques. Sur les cinq numéros qui traitent de la situation à l'étranger, entre 1960 et 2000, un seul comporte un article sur les bibliothèques françaises : Paul Poindron, «French University Libraries », dans « European University Libraries: Current Status and Developments », vol. 12, $\mathrm{n}^{\circ} 4$, April 1964. Dans quatre autres dossiers, la France est absente : "Education for Librarianship Abroad in Selected Countries" (Oct. 1963), "Metropolitan Public Library Problems Around the World" (July 1965), "Cooperative and Centralized Cataloging” (July 1967), "Library Services to Ethnocultural Minorities" (Fall 1980).

Quant à la revue Library Quarterly, je n'y ai trouvé aucun article dont le sujet soit une/des bibliothèques(s) française(s) - ce qui ne signifie pas évidemment qu'aucune mention à une/des bibliothèques(s) française(s) ne s'y trouve. Mais, si c'est le cas, c'est au mieux incident. Les bibliothèques françaises ne sont pas, en elles-mêmes, un sujet.

\section{UN VOISINAGE LOINTAIN}

On le voit, le décalage est grand entre l'intérêt porté aux voisins de chaque côté de l'Atlantique : pour les Français, les bibliothèques américaines sont le premier sujet d'intérêt dans la presse professionnelle ; pour les Américains, les bibliothèques françaises existent à peine. L'avance très grande prise par les bibliothèques américaines, leur développement beaucoup plus affirmé, leurs innovations, leurs débats, leur nombre rendent les bibliothèques des deux pays incomparables - et la différence de curiosité bien compréhensible.

Pour autant, les bibliothécaires français ne sont pas fascinés par leurs lointaines voisines : non seulement d'autres pays semblent les intéresser plus vivement, mais encore leur vision des bibliothèques américaines est assez critique.

\section{Un monde multipolaire}

L'univers des bibliothèques, tel que le dessine la presse professionnelle française, est principalement anglo-saxon ${ }^{90}$. On l'a vu, si les bibliothèques

90. Sur ce point, on se reportera à David-Georges Picard. « Un modèle anglo-saxon ?». Quel modèle de bibliothèque?, op. cit. 
américaines y occupent le premier rang, ce sont les pays européens anglosaxons qui viennent ensuite : la Grande-Bretagne, l'Allemagne, les PaysBas, le Danemark. Dans cette Europe occidentale, il est frappant de constater la quasi-absence des pays latins (catholiques). Après ce premier groupe, il faut citer un autre ensemble de pays, ceux de derrière le rideau de fer : l'URSS (puis la Russie) mais aussi les pays d'Europe de l'Est (au premier rang desquels la Tchécoslovaquie et la Pologne). Enfin, un troisième pôle géographique est représenté par l'Afrique, particulièrement les (encore ou anciennement) colonies françaises.

Si l'on considère que la connaissance réelle, ici le voyage d'études, est plus efficace et montre plus d'intérêt que la connaissance écrite, on peut affiner ce planisphère en la recentrant encore davantage. Trois pays, en effet, accueillent la quasi-totalité des voyages dont il est rendu compte dans le Bulletin d'informations de l'ABF ${ }^{91}$ : la Grande-Bretagne, le Danemark et l'Allemagne. La proximité n'explique pas tout : les Pays-Bas, la Suisse, l'Italie sont plus proches que le Danemark...

À l'appui de ce tropisme européen, on peut aussi signaler que deux seuls ouvrages, dans la collection « Bibliothèques » des Éditions du Cercle de la Librairie, concernent spécifiquement des bibliothèques étrangères : Bibliothèques publiques en Europe (1992), qui, logiquement, ne parlent d'aucune bibliothèque américaine, et Nouvelles Alexandries : les grands chantiers de bibliothèques dans le monde (1996), où l'on traite d'une bibliothèque américaine, la San Francisco Public Library.

L'intérêt manifesté pour les écrits sur les bibliothèques américaines doit donc être nuancé par l'appétit, la curiosité manifestés pour les bibliothèques du nord de l'Europe. La connaissance et l'intérêt pour les bibliothèques américaines sont surtout livresques, l'appétit et la curiosité pour les bibliothèques européennes sont concrets. L'intérêt porté aux bibliothèques américaines, au demeurant, n'exclut pas une certaine ignorance.

\section{Ignorances et incompréhensions}

La première apparition des bibliothèques américaines dans le Bulletin d'informations de l'ABF date de 1955. Plus précisément, il s'agit de l'apparition d'un bibliothécaire américain, Lionel McColvin, dont l'intervention est signalée dans le compte rendu des travaux de la Fédération interna-

91. À part quelques comptes rendus de bourses Fulbright (qui faussent les statistiques), le $B B F$ publie très rarement de comptes rendus de voyages d'études. 
tionale des associations de bibliothécaires (FIAB-IFLA) : "Section des bibliothèques populaires. A adopté avec quelques modifications de détail le memorandum présenté par Marc Colvin [sic] et destiné à étudier les conditions qui doivent permettre dans les différents pays le développement des bibliothèques de lecture publique. Cet exposé est un peu alourdi par des considérations philosophiques et théoriques concernant le droit à la lecture. ${ }^{92}{ }^{\prime}$

On peut comprendre que l'intérêt pour les valeurs qui fondent l'action des bibliothèques publiques ne soit pas partagé par tous les collaborateurs du Bulletin d'informations de l'ABF. Il est plus étonnant de constater l'incompréhension ou, même, l'ironie que suscite le fonctionnement des bibliothèques américaines. Ainsi, le compte rendu admiratif peut reposer sur un malentendu, l'organisation des bibliothèques du comté de Montgomery étant qualifiée de " très démocratique grâce aux comités consultatifs locaux ${ }^{93} »$, ou sur un tel sentiment d'étrangeté qu'on voit bien qu'aucun enseignement n’en sera tiré : « On est frappé partout par l'abondance des fonds, notamment des livres de référence, et du personnel, par la qualité du service de renseignement, l'importance de la fréquentation $\mathrm{du}$ public, les nombreux catalogues et des moyens bien supérieurs aux nôtres. ${ }^{94}$ » L'accord avec le projet américain est rarement complet : Myriam Foncin conclut une critique élogieuse de l'ouvrage de McColvin (The Chance to read, 1950) par ces lignes : « Il est bien certain que des bibliothécaires français n'accepteront pas en bloc la définition que M. McColvin donne de la bibliothèque de lecture publique, définition qu'il emprunte à Robert $\mathrm{D}$. Leigh. La plupart, en tout cas, intervertiront l'ordre des facteurs, assignant comme premier objectif à la bibliothèque l'enrichissement personnel de l'individu et non sa formation civique et sociale. ${ }^{95}$ "

S’il s'agit encore d'un modèle, il est, on le voit, considéré comme lointain, voire inaccessible.

La vision un peu académique des relations entre les deux mondes professionnels que j'ai présentée ici peut être nuancée par le côté positif des relations entre les deux associations professionnelles, l'ABF et l'ALA, ces dernières années.

92. «Compte rendu de la réunion annuelle de la Fédération internationale des associations de bibliothécaires, Zagreb, 1954 ", non signé, Bulletin d'informations de l'ABF, n 16, mars 1955.

93. Odile Altmayer, "Compte rendu du congrès de la FIAB, suivi d'un voyage d'études au Canada et aux USA », Bulletin d'informations de l'ABF, $\mathrm{n}^{\circ} 58,1^{\text {er }}$ tr.1968.

94. André Thill, à propos de la New York Public Library, Bulletin d’informations de l'ABF, $\mathrm{n}^{\circ} 87,2^{\mathrm{e}}$ tr. 1975.

95. Bulletin d'informations de l'ABF, $\mathrm{n}^{\circ} 23,1957$. 
En 1998, l'ALA a apporté son soutien à l'ABF dans sa protestation contre les agissements des élus du Front national. En 2003, l’ABF a apporté son soutien à l'ALA dans sa lutte contre l'USA Patriot Act : " L'Association des Bibliothécaires Français s'associe à la Résolution votée par l'ALA en janvier 2003 pour demander un amendement du Patriot Act restituant aux usagers des bibliothèques le droit de s'informer et de se cultiver à l'abri de toute investigation non fondée. ${ }^{96}$ » En 2005, l'ancienne présidente de l'ALA, Nancy Kranich, vient apporter des informations au congrès de l'ABF, à Grenoble, sur la lutte des bibliothécaires américains contre le Patriot Act.

$\mathrm{Au}$ long du siècle, au moins, la solidarité professionnelle ne se dément pas.

\section{Amitié et méfiance}

L'intérêt, la connaissance qu'ont les bibliothécaires français pour les bibliothèques américaines ne peuvent être tout à fait étrangers à l'intérêt, la connaissance qu'ils ont des États-Unis eux-mêmes. Tout en renvoyant aux nombreuses publications qui évoquent ces relations ${ }^{97}$, je n'évoquerai ici que le monde du livre. On peut, en effet, soutenir l'une comme l'autre thèse, celle de liens amicaux comme celle d'une distance assumée - une «fascination réticente », pour reprendre la formule de Jacques Portes.

Quelques exemples récents de liens amicaux, positifs : la parution de la correspondance entre André Gide et Jacques Schiffrin ${ }^{98}$, le voyage de Bernard-Henry Lévy au travers des États-Unis en 2005 pour répondre à une commande de The Atlantic Monthly ${ }^{99}$, une éphémère tendance de George Bush Jr à citer Tocqueville ${ }^{100}$, la nomination d'un Monsieur Livres à l'ambassade de France à New-York, le succès des festivals du livre consacrés à la littérature américaine (Vincennes, Aix-en-Provence), la réunion d'historiens américains à la BnF à l'été 2004, les Belles Étrangères consa-

96. Motion adoptée par l'Assemblée générale de l'ABF, le 15 juin 2003.

97. Parmi les plus récentes, citons L'Amérique dans les têtes, sous la dir. de Denis Lacorne, Hachette, 1986, Philippe Roger, L'ennemi américain, Éditions du Seuil, 2002, ou, de l'autre côté, Richard Z. Chesnoff, The Arrogance of the French: Why They Can't Stand us and Why the Feeling is Mutual, Sentinel, 2005.

98. Gallimard, 2005.

99. Publié en anglais sous le titre American Vertigo, chez Random House, le recueil des articles de BHL a suscité dans la presse américaine une kyrielle de critiques négatives (sur les écrivains français « courts sur les faits et longs sur les conclusions ») et de questions peu aimables : « Estce comme ça que les Français parlent ou réservent-ils ça aux livres sur l'Amérique ? " (Le Monde, 10 février 2006) La traduction française, sous le même titre, est parue en mars 2006 chez Grasset. 100. The New York Times, 14 March 2005. 
crées aux écrivains américains en novembre 2009, le succès en France comme partout dans le monde du Da Vinci Code, ou des ouvrages de Philip Roth ou Paul Auster, Don DeLillo, Jim Harrison ou David Payne, l'intérêt toujours vif pour les ouvrages des intellectuels américains comme Robert Darnton, Robert Paxton, André Schiffrin, Jeremy Rifkin ou Noam Chomsky, ou les ouvrages de chercheurs américains sur la France, comme ceux de John Baldwin, Kristin Ross ou Paul Rabinow ${ }^{101}$. Ou ce titre du Monde, à propos de la pénurie de passeports biométriques : « 500000 Français privés d'Amérique » (26 janvier 2006). Privés d’Amérique !

Cet attrait, cette curiosité pour l'Amérique, ses romanciers, ses intellectuels, étonnent quelquefois les Américains. Ainsi, le romancier Percival Everett, invité en 2004 du festival America de Vincennes, se déclare « impressionné et ému par la manière dont les lecteurs français adoptent les lettres américaines. Je ne peux pas imaginer pareille manifestation aux États-Unis et certainement pas à l'échelle de ce festival. La différence, celle qui permet la manifestation de Vincennes, est que les Français de manière générale aiment les livres, ils respectent les écrivains, ils chérissent les intellectuels, je rêverais de pouvoir dire la même chose de l'Américain moyen. ${ }^{102}$ » Les traductions ne représentaient que $3 \%$ de la production éditoriale américaine en 2003 < http://www.bief.fr >.

Sur le versant négatif, la diminution des traductions de livres - dans un sens comme dans l'autre. Un éloignement croissant des deux mondes intellectuels, à en croire Perry Anderson, qui fustige l'absence de curiosité pour les idées étrangères : «Un pays qui n’a pratiquement rien publié de Fredric Jameson ou de Peter Wollen et n'a même pas pu trouver un éditeur pour traduire Age of Extremes d'Eric Hobsbawn risque de faire figure d'arrière-garde dans l'échange international des idées. ${ }^{103}$ »

Mais aussi, bien sûr, les séquelles de la guerre d'Irak et, plus généralement, le refus de l'approche unilatérale de l'administration américaine. Plutôt qu'une opposition (que le mot « anti-américanisme » résumerait ${ }^{104}$ ),

101. John Baldwin, Paris 1200, Aubier, 2006 ; Kristin Ross, Rouler plus vite, laver plus blanc : modernisation de la France et décolonisation au tournant des années soixante, Flammarion, 2006 ; Paul Rabinow, Une France si moderne : naissance du social (1800-1950), Buchet-Chastel, 2006.

102. Libération, 16 octobre 2004.

103. Perry Anderson, La pensée tiède : un regard critique sur la culture française, Éditions du Seuil, 2005, p. 91. Inversement, Perry Anderson louange la curiosité française pour les littératures étrangères.

104. S’il correspondait à une réalité, ce dont Pascal Ory doute. Pourtant, on peut en décrire les préjugés stéréotypiques, ce que fait Philippe Roger en citant Régis Debray (le Régis Debray de 1992) : « le consumérisme sans fin ni répit, le tout-marchandise et la croyance dans la neutralité de la technique, la disparition du citoyen sous le consommateur, l'insensibilité au tragique, la 
c'est la méfiance qui semble surtout animer le monde du livre et des bibliothèques, la volonté de se défendre contre une menace d'hégémonie. Un excellent exemple de ce rejet est la réaction de Jean-Noël Jeanneney, alors président de la BnF, à l'annonce par Google de son projet de numériser et de rendre accessibles 15 millions d'ouvrages : " Le vrai défi est ailleurs, et il est immense. Voici que s'affirme le risque d'une domination écrasante de l'Amérique dans la définition de l'idée que les prochaines générations se feront du monde [...]. La production scientifique anglosaxonne, déjà dominante dans une quantité de domaines, s'en trouvera forcément survalorisée, avec un avantage écrasant à l'anglais par rapport aux autres langues de culture, notamment européennes [...]. Lorsque s'est posée, depuis la seconde guerre mondiale, du côté du cinéma puis de l'audiovisuel, la question de la riposte française à la domination américaine, vouée, si l'on n'avait pas réagi, à opprimer chez nous toute production originale, une première réaction a été de protectionnisme, selon un système de quotas, dans les salles puis à la télévision. Cela n'était pas illégitime et a été partiellement efficace. Mais, dans le cas qui nous occupe, cette stratégie se révèle, compte tenu de la nature de la Toile, impossible. Reste donc la seconde, qui a fait ses preuves sur nos divers écrans : celle de la contre-attaque, avec un soutien positif à la différence. ${ }^{105}$ »

L'élection, en 2008, de Barack Obama et son corollaire, la fin de l'ère Bush, ont modifié ce paysage ${ }^{106}$. Mais, quelle que soit la durée de l'état de grâce que l'élection de Barack Obama a suscité, quelle que soit la durée de cette « Obamania » française, il n'en demeure pas moins que le besoin de se protéger ${ }^{107}$, de protéger les productions nationales du monde anglosaxon reste très largement partagé. Ainsi Patrick Bazin, directeur de la Bibliothèque municipale de Lyon, conforte l'analyse de Jean-Noël Jeanneney : « En effet, explique-t-on, en monopolisant les outils qui permettent de structurer les documents et d'y accéder, les États-Unis vont,

confusion du public et du privé, le culte de la réussite et de l'argent, l'impératif de la réduction de la vie humaine à un ensemble d'activités profitables, etc., etc. " (L'ennemi américain, op. cit., p. 15) Bernard-Henri Lévy se livre au même exercice dans American Vertigo (op. cit., p. 19). André Kaspi souligne que "l'antiaméricanisme reste une valeur sûre dans notre pays. Que nous en soyons conscients ou non, il imprègne notre conversation et notre réflexion. Il constitue l'un des fondements de la culture française. » (Les États-Unis d'aujourd'hui, mal connus, mal aimés, mal compris, Perrin, 2004, p. 33)

105. Jean-Noël Jeanneney, « Quand Google défie l’Europe », Le Monde, 23 janvier 2005.

106. Les enquêtes d'opinion parlent d'elles-mêmes : en 2008, le soutien de l'opinion française à l'action de George Bush s'élevait à $11 \%$; en 2009, le soutien à l'action de Barack Obama à $88 \%$ (Étude Transatlantic Trends, Le Monde, 10 septembre 2009).

107. À la base de la politique dite de l'exception culturelle. 
quand bien même ils ne le voudraient pas, imposer leur point de vue à des œuvres de l'esprit qu'ils n'ont pas produites eux-mêmes et enlever aux peuples du monde la maîtrise de leur propre mémoire culturelle, voire de leur mode de pensée actuel. ${ }^{108}$ "

La langue comme culture. Écoutons George Steiner sur ce point : «Chaque langue contient, articule et transmet non seulement une charge unique de mémoire vécue, mais encore une énergie élaboratrice de ses temps futurs, une potentialité pour demain. La mort d'une langue est irréparable, elle diminue les possibilités de l'homme. Rien ne fait peser sur l'Europe une menace plus radicale - à la racine - que la progression exponentielle et détergente de l'anglo-américain et l'uniformité des valeurs et de l'image du monde que cet espéranto dévorant apporte avec lui. ${ }^{109}$ »

Dans ce climat de défiance, d'opposition, de désamour (ou de déception amoureuse) ou de retour de flamme, l'étude de la filiation ou de la désaffiliation doit remonter aux sources - à la « matrice bibliothécaire » pour reprendre la formule de Robert Damien. 
CHAPITRE II DEUX HISTOIRES, DEUX HÉRITAGES 


\section{CHAPITRE II DEUX HISTOIRES, DEUX HÉRITAGES}

a lecture a une histoire - « Lire n'est pas un invariant anthropolo-
gique sans historicité », dit Roger Chartier.

Les bibliothèques ont une histoire (et une géographie).

Les bibliothèques publiques ont une histoire - à la fois commune et très différente. Chaque pays, dans son histoire politique, sociale, religieuse, culturelle, intellectuelle développe, a développé un modèle particulier de bibliothèque, même si certains grands traits sont communs à beaucoup d'entre eux. Ou, plus exactement, même si certaines grandes familles peuvent être distinguées. Ainsi, pour les pays occidentaux, les familles «doctrinale » et " libérale », baptisées et décrites par Michel Melot : d'un côté, le modèle doctrinal, qui prend essor en Europe chez les humanistes italiens et les libres penseurs français et qui, de Montaigne à Naudé, fait de la bibliothèque une construction intellectuelle, " une institution fragile, contestée, une arme dans la lutte d'une libre pensée », " une bibliothèque choisie [...] par des penseurs qui devenaient, en quelque sorte, les auteurs de leur collection. » De l'autre côté, le modèle libéral, « où l'on peut voir l'œuvre de la Réforme, animée par un rapport nouveau au savoir qui n'est plus ni doctrinal ni anecdotique, un savoir intégré dans la pratique religieuse, un devoir de connaissance. À partir de cette époque et de ce lieu, la bibliothèque n'est plus un puits au fond duquel il faut aller chercher la science, mais une rivière qui irrigue et fertilise la vie quotidienne et permet à chacun de gagner son salut. ${ }^{110}$ » D’un côté (disons ici, du côté français), une bibliothèque qui tient un discours sur le monde et où « la primauté de la politique de l'offre » s'appuie sur le rôle collectionneur des bibliothécaires ; de l'autre côté (donc, du côté américain), une bibliothèque où la connaissance sert à la maîtrise du monde, qui doit « répondre mot pour mot à toutes les demandes » et à qui « un empirisme

110. Michel Melot. "Pour une géopolitique des bibliothèques », in Anne-Marie Bertrand, Anne Kupiec, Ouvrages et volumes : architecture et bibliothèques. Éditions du Cercle de la Librairie, 1997, p. 99-100. 
élevé à la dignité de doctrine [peut] donner sa véritable dimension », écrit encore Michel Melot.

Le poids de la tradition religieuse, Alexis de Tocqueville, évidemment, l'avait déjà souligné : « C’est la religion qui a donné naissance aux sociétés anglo-américaines : il ne faut jamais l'oublier ; aux États-Unis, la religion se confond donc avec toutes les habitudes nationales et tous les sentiments que la patrie fait naître. ${ }^{111}$ " Les bibliothèques, elles aussi, ont été façonnées par cette influence. L'analyse de Blandine Kriegel propose un raccourci éclairant de l'origine calviniste des bibliothèques « libérales » : « L'aboutissement du calvinisme radical, c'est une Église libre dans un pays libre où, de surcroît, chacun a le droit d'interpréter, à son gré, la parole de Dieu [...]. Le jardin d’Eden a été abandonné, et la seule chance de l'homme, son salut, réside dans le labeur du travail et dans les affaires de la cité, dans l'ordre économique et politique, dans la possibilité de bâtir, par l'exercice de la raison et par la tension de l'effort corporel, un monde plus puissant et plus prospère, où il est exclu de trouver, sur terre, la maison de Dieu. ${ }^{112}$ " Et aussi : " Le nihilisme radical restitue l'homme à une liberté aussi revigorante que le climat froid de l'Europe du Nord a été fatal à Descartes. Elle a conduit à conquérir l'Amérique du Nord, à abattre les bouleaux et à épierrer les champs de la NouvelleAngleterre au moment même où les Espagnols pillaient les trésors d'une nature généreuse et dispendieuse. » Elle a conduit les Américains à concevoir des bibliothèques publiques, ouvertes et utiles, au moment où Espagnols et Français thésaurisaient le trésor inaccessible des siècles passés.

Le protestant qui fait fructifier les dons du Seigneur dans ce monde " intégralement occupable » (Marcel Gauchet ${ }^{113}$ ), l'homo œconomicus, « l'homme rationnel et industrieux » (Locke) qu'appelait nécessairement ce continent à découvrir et à peupler, ont une approche particulière du livre, du savoir et, donc, de la bibliothèque. Approche non médiée, approche utilitaire : non médiée car l'accès direct au Livre est la norme dans une religion qui se fonde sur « le sacerdoce de tous les croyants » et prône la

111. Alexis de Tocqueville. De la démocratie en Amérique, tome 2, Gallimard (Folio), 1961, p. 17.

112. Blandine Kriegel. Philosophie de la République. Plon, 1998, p. 145.

113. Cette transformation de "l'être-au-monde », Marcel Gauchet, d'ailleurs, la constate bien avant «l'esprit du protestantisme », dans un Moyen Âge finissant dont le démarrage économique permet « la densification des êtres, l'intensification de leur activité, l'accumulation des biens » : un " immense mouvement souterrain a peu à peu partout imposé l'optimisation active de la sphère terrestre en lieu et place de l'ancienne soumission limitative à l'intangible. " (Le désenchantement du monde, Gallimard, Folio, 1985, p. 175 et 155). 
responsabilité individuelle ${ }^{114}$; utilitaire car la réussite ici-bas suppose une prise sur le monde et l'acquisition des savoirs nécessaires. Le faible développement des bibliothèques du sud de l'Europe (latines, catholiques), l'écart de temporalité entre le développement des bibliothèques québécoises (catholiques) et du Canada anglophone (protestant) ${ }^{115}$ sont des manifestations bien connues de ce différentiel dû à l'histoire religieuse.

Michel Melot brièvement, Jean Hassenforder ou Robert Damien plus longuement ont à leur tour souligné le poids des héritages catholique ou réformé sur le rapport au livre et à la bibliothèque. Car la bibliothèque dans son expansion sans fin " atteste de l'absence d'une vérité totale et unique et en conséquence, de l'insuffisance de chaque livre puisqu'il y faut toujours ajouter ", analyse Robert Damien ${ }^{116}$. La bibliothèque serait la conséquence de l'insuffisance de la Bible, idée sacrilège qui explique l'hostilité structurelle que la tradition catholique oppose aux bibliothèques : "La désacralisation consiste à admettre la pluralité abondante des livres, sans qu'aucun puisse revendiquer le privilège ontologique d'être. Le Livre où tout se tient et qui contient le tout. Il s'agit bien de se délivrer de cette névrose du texte référentiel et vénéré. Le fantasme religieux du Texte est l'obstacle épistémologique qui empêche le développement séculier de la bibliothèque et de son savoir déposé. La logique du Livre est en effet de réduire la bibliothèque au texte unique de la Bible. ${ }^{117}{ }^{\text {» }}$

Ainsi, les bibliothèques sont des artefacts construits par l'histoire ${ }^{118}$. En particulier, on le voit, par l'histoire religieuse. Ici, tout en intégrant cette analyse, je traiterai principalement d'autres héritages, les héritages politiques qui ont aussi, on s'en doute, une importance matricielle. Pour

114. « Les boutiquiers puritains qui avaient appris à parler à Dieu devant leurs apprentis, leur femme et leurs enfants étaient déjà sur la voie de l'autonomie. Si inférieur que pût être, au temple, leur rang parmi les fidèles, ils trouvaient chez eux la reconnaissance gratifiante de leur dignité et de leur valeur. » (Elizabeth Eisenstein. La révolution de l'imprimé à l'aube de l'Europe moderne. Hachette, 1991, p. 204)

115. L'historien des bibliothèques québécoises, Marcel Lajeunesse, insiste sur le poids du clergé comme cause du retard des bibliothèques québécoises : une vision de l'individu comme « conscience à diriger » et « fidèle à édifier » a, dit-il, créé « une méfiance certaine envers la lecture et les institutions qui pouvaient l'assurer ». (Marcel Lajeunesse. Lecture publique et culture au Québec, XIX et $X X^{e}$ siècles. Presses de l'université du Québec, 2004, p. 218). Le développement des bibliothèques publiques québécoises date de la Révolution tranquille, dans les années 1960, celui des bibliothèques des provinces anglophones est contemporain de celui des Public Libraries américaines, dans la seconde moitié du XIX ${ }^{\mathrm{e}}$ siècle.

116. Robert Damien. Bibliothèque et État : naissance d'une raison politique dans la France du XVII siècle. PUF, 1995, p. 16.

117. Robert Damien. Le conseiller du Prince de Machiavel à nos jours : genèse d'une matrice démocratique. PUF, 2003, p. 182.

118. "The library is an artifact of our historical landscape". Kenneth Carpenter, Thomas Augst. "The History of Libraries in the United States". Libraries \& Culture, Winter 2003. 
comprendre l'identité des bibliothèques publiques aujourd'hui, il faut nécessairement remonter à leur naissance, à leur socle et à leurs fondations.

\section{AUX ÉTATS-UNIS}

Si l'histoire des bibliothèques en France est parsemée de légendes et de figures héroïques, cette caractéristique est encore bien plus vraie des bibliothèques américaines. C'est ainsi non seulement les origines de la Public Library qui sont narrées dans des pages enthousiastes, mais aussi les valeurs, l'éthique, la Library Faith, qui animent les bibliothécaires américains, qui font l'objet de développements innombrables et vertueux - au point d'avoir suscité un courant historique critique. Cet enthousiasme, voire cette emphase, font partie du regard porté sur la Public Library, de la représentation qu'en a la population américaine. Ils magnifient l'histoire et le présent. Ils sont peut-être l'une des premières différences sensibles entre bibliothèques françaises et bibliothèques américaines.

\section{La naissance de la Public Library}

De même que Naudé serait (à tort) considéré comme l'inventeur de la bibliothèque publique, de même Benjamin Franklin est considéré à tort comme l'inventeur de la Public Library. À Philadelphie, avec son club d'amis, son Junto, il fut cependant un des premiers à décider de mettre en commun des livres, pour le bien de tous : en ce sens, il peut être considéré comme un des initiateurs du " concept de propriété collective » des livres (Jesse Shera ${ }^{119}$ ). Sa Library Company of Philadelphia, fondée en 1732, est un des premiers exemples des social libraries, bibliothèques associatives destinées à donner accès à des livres par la mise en commun de ressources, pour nourrir la curiosité, la soif de savoir et l'organisation de débats - et créées à l'initiative d'individus ou de groupes d'individus, à l'image des book clubs anglais. La forme associative, si importante pour la future organisation des bibliothèques, n'est, au départ, qu'une commodité, le système le plus efficace pour mener un projet commun. De la

119. Jesse Shera est l'auteur des Foundations of the Public Library. The Shoe String Press, 1965, et, avec Sidney Ditzion, (auteur de Arsenals for a Democratic Culture: A Social History of the American Public Library Movement in New England and the Middle States from 1850 to 1900. ALA, 1947), un de nos principaux guides dans cette histoire. 
même façon, dans la jeune Amérique qui conquiert son continent, la construction des routes, l'alimentation en eau ou la lutte contre les incendies sont prises en charge par des individus regroupés en associations. Ces bibliothèques (collectives mais non publiques) ont connu leur âge d'or dans la première moitié du XIX ${ }^{e}$ siècle. En 1850, on en comptait plus d'un millier en Nouvelle-Angleterre. Mais leur espérance de vie excédait rarement celle de leurs fondateurs : une fragilité récurrente était liée au caractère volontaire, et forcément éphémère, du groupement des fondateurs de la bibliothèque ${ }^{120}$.

L'évolution de ces premières activités vers une forme " publique » est rendue possible par l'urbanisation rapide de la Nouvelle-Angleterre et par l'amélioration du niveau de vie. La naissance officielle, consacrée, de la Public Library a lieu à Boston en 1854, premier exemple célèbre (et célébré) où une bibliothèque est prise en charge par les pouvoirs publics - et, donc, premier exemple d'une bibliothèque financée par l'impôt (taxsupported library) ${ }^{121}$. Boston, alors la quatrième ville des États-Unis (93000 habitants) et la plus importante pour le commerce des livres, est dans une phase de croissance rapide et de centralisation de ses services. Audelà du pittoresque et des aléas de cette création ${ }^{122}$, il faut retenir la forme sous laquelle cette bibliothèque a été créée. Il s'agit de la réunion sous les auspices publiques de plusieurs social libraries. Le service est municipal, mais doté d'une instance de gouvernement autonome. Le financement est public et privé. Quel sens donner à ces choix ?

Réunir les social libraries sans perdre le contrôle de l'institution : voilà les raisons pour lesquelles c'est un fonctionnement proche du fonctionnement associatif qui est adopté à Boston, et qui le sera presque partout, car il est devenu une des clés de l’identité de la Public Library. La créa-

120. La moitié n'a pas duré plus de 35 ans.

121. Les historiens avertis rappellent que la première bibliothèque financée sur budget public a vu le jour en 1833 à Peterborough (New Hampshire), de façon éphémère. La création de la Public Library de Boston n'en demeure pas moins emblématique et est reconnue comme telle. Ainsi, dans le National Millenium Time Capsule, recueil d'objets conservé aux Archives depuis 2001 et destiné à " donner au président américain de 2101 une idée de la culture et des valeurs que les Américains du xx siècle partageaient », figure une carte d'usager de la Boston Public Library (American Libraries on line, posté le 11 décembre 2000).

122. Il faudra plus de dix ans aux promoteurs de cette idée pour vaincre l'apathie de la population, dix ans de débats, de lutte d'influence et de lentes avancées réglementaires. Jesse Shera évoque "the combined influence of many civic leaders to overcome the inertia of popular apathy". La création de la bibliothèque est votée par les autorités municipales en 1848, mais son ouverture n'aura lieu qu'en 1854. L'état du Massachusetts a autorisé en 1851 les villes de l'état à lever l'impôt pour créer et entretenir des bibliothèques (State Public Library Law). 
tion d'un Board of Trustees est votée dès 1852, il sera composé de deux membres du Common Council et de cinq citoyens désignés par le maire.

Comme pour les social libraries, la participation financière (volontaire) des citoyens est un mode usuel de financement. À quoi s'ajoute, grande nouveauté, le financement public : l'impôt. Le financement public n'est possible, souhaitable, accepté que parce que la bibliothèque est reconnue comme utile à l'ensemble de la population (parce qu'elle est un service public, dirait-on en France). C'est pourquoi le but de la bibliothèque, la population au bénéfice de qui elle est créée sont des sujets d'importance capitale - et abondamment étudiés. Pour en rester à l'exemple de Boston, trois types d'arguments sont avancés pour justifier l'ouverture à l'ensemble de la population. Le premier est que la démocratie naissante a besoin d'électeurs qui prennent des décisions éclairées. Le deuxième est que la société et l'économie ont besoin d'outils qui permettent la qualification de chacun. Enfin, le troisième est qu'il faut canaliser la rudesse et les mauvais penchants des masses ignorantes, au premier rang desquelles les nombreux immigrants qui viennent peupler l'Amérique du XIX ${ }^{\mathrm{e}}$ siècle. Reprenons cet argumentaire.

Dès le milieu du siècle, le principe démocratique est acquis par la population elle-même. " Les concepts de droits de l'homme, d'égalité politique, et d'autorité résidant dans le peuple tout entier sont désormais fermement fixés dans le credo américain. ${ }^{123}$ " L'exercice du droit de vote, libéralement attribué entre tant de mains ${ }^{124}$, doit être exercé par des citoyens conscients et informés. Les livres, et donc la bibliothèque, sont les outils de cette éducation. " Les livres étaient à la fois les catalyseurs et les guides pour l'esprit créatif qui gît dans tout être humain. Ils étaient la machinerie qui produirait des citoyens plus intelligents, mieux informés, utiles et respectables. ${ }^{125}$ » "Éclairez le peuple », demandait Thomas Jefferson. Et James Madison : «Un gouvernement populaire sans information pour le peuple, ni les moyens pour le peuple d'y avoir accès n'est qu'un prologue à une farce, à une tragédie ou les deux à la fois. Le savoir l'emportera toujours sur l'ignorance. »

La deuxième famille d'arguments est proche : cette fois ce n'est plus la démocratie, c'est la société, c'est l'économie qui ont besoin que l'ignorance soit combattue, car elles ont besoin de chefs de famille aptes à 
prendre les meilleures décisions, de fermiers compétents, d'ouvriers qualifiés. S'y ajoute le rêve américain de l'ascension sociale qui ne peut se faire que par le travail et sa propre amélioration (self-improvement) - le self-made man a besoin de bibliothèques. L'éthique du travail, le souci du rendement, la mobilisation des énergies induisent une approche utilitaire du savoir : "Il n'y a pas de place pour d'autres cultures que celles des champs, de l'atelier, de la boutique, ou du bureau. ${ }^{126}$ » La bibliothèque est un outil de formation. À ce titre, le financement public lui est dû au même titre qu'il est dû aux écoles publiques.

Enfin, type d'arguments moins positif ${ }^{127}$, il s'agit aussi d'exercer un contrôle social sur les foules de pauvres, de Noirs, d'immigrants qui peuplent, de plus en plus nombreux, les métropoles naissantes. Car les fondateurs tant loués de la Boston Public Library n'avaient pas que des buts désintéressés, dit Michael Harris ${ }^{128}$ : le débat des années 1850 entre ses fondateurs Edward Everett et George Ticknor doit être revisité. Il ne s'agit pas tant d'un désaccord technique sur le projet (une bibliothèque de référence pour Everett, une bibliothèque populaire pour Ticknor) : ni l'un ni l'autre ne sont des libéraux et ni l'un ni l'autre n'ont vraiment confiance en l'homme ordinaire (the common man). La bibliothèque largement ouverte à tous, dans l'idée de Ticknor, est le meilleur moyen de faire de tous des « hommes sobres, vertueux, conservateurs, endurants et dévots, en bref, d'en faire des hommes comme lui. ${ }^{129}$ " Le vice, la pauvreté, l'étrangeté : voilà ce que la Public Library doit contribuer à combattre. Elle sera un facteur de moralité (pour les pauvres en voie de dépravation) et d'américanisation (pour les immigrants).

Affichés, demi-avoués ou cachés, les objectifs assignés aux Public Libraries sont désormais fixés dans leurs grandes lignes. Former un peuple de citoyens, contribuer à l'élévation de chacun et au progrès de tous, intégrer le peuple si divers de ce jeune pays. Ces objectifs ont su mobiliser largement autour d'eux, grâce à la profession naissante des bibliothécaires dont l'association professionnelle, the American Library Association (ALA) créée en 1876, eut pour premier slogan dès 1879 : « Les meilleurs livres pour le plus grand nombre au moindre coût ».

126. Adrien Lherm. "Existe-t-il une culture américaine ? ", in André Kaspi, François Durpaire, Hélène Harter, Adrien Lherm. La civilisation américaine, PUF, 2004, p. 181.

127. Mais que Shera et Ditzion mentionnent clairement.

128. Michael H. Harris. "The Role of the Public Library in American Life: a Speculative Essay", University of Illinois, Graduate School of Library Science. Occasional Papers, n ${ }^{\circ} 117$, Jan. 1975. 129. Ibid. 
Ils ont cours, depuis lors, de façon à peu près intangible. Ils ont notamment été les pierres de touche de la création de si nombreuses bibliothèques dans les villes grandes et moyennes dans la seconde moitié du $\mathrm{XIX}^{\mathrm{e}}$ siècle. On les retrouve ainsi dans la création de la Chicago Public Library. Alors qu'une social library, créée en 1841 et gérée par la Young Man's Association, faisait office de bibliothèque publique, les appels à la prise en charge publique (par l'impôt) restèrent longtemps vains. En 1866, le président du Library Committee appelait la ville à reconnaître « de manière claire la nécessité et la grande valeur d'une Public Library comme instrument de développement intellectuel et de réelle prospérité. ». En 1871, le Chicago Tribune fait campagne en faveur de la création d'une taxsupported library : "L'école bénéficie de cet avantage », explique l'éditorialiste : «Il y a exactement le même besoin d'une bibliothèque publique que d'une école publique et il y a les mêmes fortes raisons pour soutenir l'une comme l'autre par des taxes levées sur la population. ${ }^{130}$ " La mobilisation des lecteurs et de quelques leaders d'opinion permit d'aboutir en 1872. L'historienne fait de cette création un exemple parfait du modèle de bibliothèque publique : « Dès son origine, cette bibliothèque fut populaire, autorisée par l'état, soutenue par l'impôt, administrée par un conseil, à caractère indépendant, service municipal promouvant l'objectif général d'éducation, de loisir et de culture dans la ville de Chicago. »

On les retrouve, ces principes de départ mais quelque peu déformés, dans la grande œuvre philanthropique de Carnegie, qui voulait à la fois rendre à la société ce qu'elle lui avait donné (de l'argent), aider les jeunes gens pauvres et méritants à s'élever dans la vie comme il l'avait lui-même fait et, par l'éducation, contribuer à maintenir l'ordre : " La connaissance fait des hommes non pas de violents révolutionnaires, mais de prudents réformistes ; non pas des destructeurs mais de soigneux progressistes. ${ }^{131}$ » Les ouvriers, les pauvres et surtout les immigrants sont l'objet avoué de ce mouvement d'acculturation qui, lié à l'idée d'intégration dans une nouvelle patrie, portera le nom d'américanisation, la bibliothèque étant ainsi désignée comme une Americanizing institution ${ }^{132}$.

130. Citations extraites de Gwladys Spencer. The Chicago Public Library: Origins and Backgrounds. University of Chicago Press, 1943, passim.

131. Cité par Michael Harris. "The Role of the Public Library in American Life", op. cit.

132. Une bibliothécaire en 1903, citée par Michael Harris. "The Role of the Public Library in American Life", op. cit. Il commente : "Les bibliothécaires semblaient croire qu'ils jouissaient d'un mandat divin pour éclairer les immigrants”. 
La fin $\mathrm{du} \mathrm{XIX}^{\mathrm{e}}$ siècle, le début du XX $\mathrm{X}^{\mathrm{e}}$ siècle, et pas seulement sous l'influence des philanthropes, sont ainsi marqués par une interprétation morale, voire moralisante, des principes démocratiques originels. Sidney Ditzion analyse cette déformation des premiers idéaux comme un paternalisme conservateur qui ira jusqu'à l'imposition de ses propres idées ou préjugés.

Autre écart, non seulement les principes sont appliqués de façon quelquefois lointaine, mais surtout la distance entre les principes affichés et la réalité des services rendus est considérable. C'est ce que montre la grande enquête de 1948, la Public Library Inquiry.

\section{La Public Library Inquiry}

Commanditée par l'ALA en 1947, financée par la Carnegie Corporation, réalisée par le Social Science Research Council sous la direction de Robert Leigh, la Public Library Inquiry mobilise pendant deux ans vingt-quatre chercheurs, sociologues, politologues, économistes, qui menèrent 19 études différentes pour apprécier le rôle de la Public Library dans les États-Unis d'après-guerre. Trois des rapports publiés nous intéressent particulièrement, le rapport général ${ }^{133}$, l'étude sur le public et ses usages ${ }^{134}$, l'analyse de la place de la Public Library dans le monde politique ${ }^{135}$. Ils analysent finement la relation entre les grands principes affichés et partagés par les responsables des bibliothèques comme par les bibliothécaires, les services effectivement rendus (et les collections effectivement proposées) et l'usage réel qui en est fait par la population.

Après la deuxième guerre, la Public Library continue à être gouvernée par les grands principes démocratiques que ses fondateurs avaient promus : proposer des collections aptes à former des citoyens éclairés et à enrichir la vie de chacun ; être un centre d'information pour toute la population ; aider et encourager enfants, jeunes, hommes et femmes dans leur formation tout au long de leur vie. Ces grands principes reposent sur la Library Faith, « la croyance en la vertu du texte écrit, en particulier du livre, dont la lecture est bonne en soi et dont sort ce qui est bon. ${ }^{136}$ » La

133. Robert D. Leigh. The Public Library in the United States: The General Report of the Public Library Inquiry. Columbia University Press, 1950.

134. Bernard Berelson, avec la participation de Lester Asheim. The Library's Public: A Report of the Public Library Inquiry. Columbia University Press, 1949.

135. Oliver Garceau. The Public Library in the Political Process: A Report of the Public Library Inquiry. Columbia University Press, 1949.

136. Robert D. Leigh. The Public Library in the United States, op. cit., p. 13. 
Public Library est donc vue d'abord comme un outil d'éducation et de formation, de self-improvement, basé sur la diffusion de livres. Ces principes, qu'élus, membres des Library Boards, citoyens, bibliothécaires, semblent toujours approuver, devraient être mis en œuvre par des collections et des services adaptés qui, eux-mêmes, devraient recevoir un accueil chaleureux, voire enthousiaste, de la population. Or, l'enquête montre que ces effets attendus ne sont pas au rendez-vous. Quarante ans avant que les bibliothèques françaises soient également confrontées par des sociologues à leurs résultats décevants au regard de leurs ambitions, les bibliothèques américaines sont mises en question.

La formation des citoyens, l'éducation après l'école, hors de l'école (after school, post-school, out of school sont également employés), la formation d'un jugement éclairé, l'intérêt pour les affaires publiques, tous ces objectifs ne peuvent s'appuyer que sur des collections de documents d'un bon niveau, habilement sélectionnés et conseillés, et sur des services de référence (nous dirions - nous disions - salles d'étude). La Public Library Inquiry montre, au contraire, des collections faibles en nombre, d'une qualité médiocre, où la fiction occupe une place de choix. « Excepté dans les grandes villes, la bibliothèque n'est pas dotée des moyens qui lui permettraient de rendre les services définis par les objectifs officiels ou issus de l'idée que dans une démocratie le peuple doit bénéficier des mêmes chances d'apprendre (equal opportunities to learn) ${ }^{137}$ " : telle est la conclusion sévère de Robert Leigh.

Ces bibliothèques, même infidèles à leurs principes fondateurs, sontelles aussi fréquentées que leur image le laisse supposer ? Non. Après correction des données statistiques ${ }^{138}$, c'est le chiffre de $10 \%$ d'usagers dans la population adulte qui est obtenu si on compte les personnes ayant emprunté au moins un livre dans le mois précédent, $18 \%$ si l'on prend en compte les emprunts dans les douze mois précédents. Pour les enfants, les estimations correspondantes sont respectivement de 33 et $50 \%{ }^{139}$.

De son côté, Bernard Berelson met en évidence l'écart entre l'image positive qui est attachée à la Public Library et son faible usage. «Bien que la plupart des adultes aiment la Public Library, la plupart d'entre eux ne la fréquentent pas. » Il cite même un sondage mené dans des grandes villes, où était posée la question : «Est-ce que ce serait très différent 
1. Pour vous, 2. Pour la ville, s'il n'y avait pas de Public Library dans la commune?»

\begin{tabular}{|l|r|r|}
\hline & Pour vous & Pour la ville \\
\hline Très différent (great deal) & $21 \%$ & $78 \%$ \\
\hline Un peu différent (quite $\boldsymbol{a}$ bit) & $16 \%$ & $16 \%$ \\
\hline Pas vraiment différent (not much) & $61 \%$ & $2 \%$ \\
\hline Sans opinion & $2 \%$ & $4 \%$ \\
\hline
\end{tabular}

Commentaire de Bernard Berelson : " Le grand public semble considérer qu'il est bien que la population bénéficie d'une Public Library - et que les autres gens la fréquentent ${ }^{140}{ }$.

Les personnes qui fréquentent la bibliothèque sont surtout des jeunes (les trois-quarts des utilisateurs ont moins de 35 ans), des étudiants, des personnes diplômées et des femmes au foyer. La fréquentation de la bibliothèque chute brutalement à l'âge où les jeunes sortent du système scolaire. " La Public Library sert les classes moyennes, définies par leur profession ou par leur statut économique, plus que les classes supérieures ou populaires. » Toutes analyses que l'on retrouvera en France dans les années 1980.

Bernard Berelson le dit radicalement. La Public Library touche un petit nombre d'usagers et c'est normal : « On peut raisonnablement avancer que dans les conditions actuelles, la fréquentation doit être faible et que la bibliothèque devrait être organisée pour le petit nombre de gens qui, dans la population, peuvent faire un usage "sérieux" des collections de la bibliothèque. ${ }^{141}$ " Ce serait renoncer à toucher toute la population ? Oui : « Le bibliothécaire devrait peut-être redéfinir explicitement l'objectif de toucher l'ensemble de la population pour offrir à la minorité des "sérieux" utilisateurs de livres et de chercheurs d'information les livres dont ils ont besoin. »

Quelle fut la réception de cette enquête ? Dans un premier temps, les bibliothécaires manifestent de la surprise, de l'incompréhension ou du désaccord devant cet exercice de dévoilement ${ }^{142}$. L'un d'eux, Ralph Munn, 
alors directeur de la Carnegie Library de Pittsburgh, décrit l'impact de l'enquête sur le public (le travail de Berelson) comme « ayant fracassé nos egos et bouleversé tout le folklore dans lequel nous avons été éduqués. ${ }^{143}$ " Alors que l'on attendait les habituelles louanges tressées à la Public Library et à son rôle démocratique, le constat de la faible fréquentation par un public non représentatif de la population est un choc. Sur le long terme, les réactions les plus vives concernent, on s'en doute, l'hypothèse d'offrir en priorité un service de qualité aux "sérieux » utilisateurs de livres, d'admettre de ne servir qu'une minorité de lecteurs éclairés, leaders d'opinion, par qui, indirectement, l'ensemble de la population serait touchée. Considérée comme élitiste ${ }^{144}$, cette proposition fut ardemment combattue au fil des ans.

Service to all, servir tout le monde : cet objectif fondamental (fondateur) n'a en rien été modifié ni par le constat de l'écart entre les objectifs et les résultats ni par la proposition de réorientation vers un public cible. En 1956, par exemple, les standards établis par la section des Public Libraries de l'ALA affirment d'entrée que « les services de la Public Library devraient être disponibles pour tous » et que chaque individu doit avoir accès à une Public Library dans sa communauté. La version de 1966 de ces standards reprend la même formulation. À partir des années 1980, c'est une vision différente du public qui est affichée, où la valeur du Service to all se déplace vers le service de chacun, tout en refusant de ne servir que des segments de la population. En 1982, l'ALA adopte officiellement le texte « The Public Library: Democracy’s Resource », qui commence par ces mots : « Les Public Libraries offrent gratuitement l'accès à leurs collections et à leurs services à tous les membres de la communauté sans considération de race, de citoyenneté, d'âge, de niveau scolaire, de statut économique ou de quelque autre qualité ou condition. »

Pendant une trentaine d'années, cette enquête, ces débats, ces discussions ont ainsi profondément influencé l'évolution des bibliothèques américaines, tant sur la clarification des principes de base (desservir l'ensemble de la population) que sur les moyens d'y parvenir (par des bibliothèques plus fortes car regroupées au sein de réseaux). La Library Faith sort de

143. Cité par Douglas Raber et Mary N. Maack, "Scope, Background and Intellectual Context of the Public Library Inquiry", Libraries \& Culture, Winter 1994.

144. Douglas Raber et Mary N. Maack soulignent que cette proposition reprenait simplement l'analyse de la naissante sociologie de la communication sur l'influence à deux niveaux, des médias vers les leaders d'opinion et des leaders d'opinion vers la population. Les bibliothécaires y virent pourtant une atteinte intolérable au projet démocratique, du « snobisme intellectuel » complètement déplacé dans une institution publique. 
cet épisode renforcée et clarifiée : la Public Library Inquiry a servi de détonateur pour la relance d'une ambition pour les Public Libraries. Comme le fera en France, en son temps, le comité inter-ministériel de 1966-1967.

\section{Bibliothèques et démocratie}

Que la bibliothèque soit un élément important de la vie démocratique, voilà une assertion qui rassemble des deux côtés de l'Atlantique. Les Américains le disent peut-être avec plus d'emphase : « Les démocraties ont besoin des bibliothèques ", "Les bibliothèques sont des institutions suprêmement démocratiques ", ou bien "La relation symbiotique entre bibliothèques et démocratie est souvent célébrée ". Ils citent souvent, avec ferveur, ces phrases de Franklin D. Roosevelt prononcées en 1942 : « Dans cette première année de la guerre, nous avons vu grandir le pouvoir des livres comme armes. Cela est justifié car la guerre des idées a besoin de livres comme une guerre navale a besoin de bateaux [...]. Les bibliothèques sont directement et immédiatement impliquées dans le conflit et ceci pour deux raisons : d'abord, parce qu'elles sont essentielles au fonctionnement d'une société démocratique ; ensuite parce que le conflit actuel concerne l'intégrité du savoir, la liberté intellectuelle et même la survie de notre culture et que les bibliothèques sont de grands outils de savoir, de grands dépôts de culture et de grands symboles de la liberté intellectuelle. ${ }^{145}$ »

Il n'y a pas qu'une différence de ton entre Français et Américains à ce propos. Il y a, surtout, une différence de contenu. Pour les Français, la démocratisation des bibliothèques c'est atteindre un public plus nombreux et dont la composition reflète mieux celle de la société. Pour les Américains, les bibliothèques dans la vie démocratique c'est, d'une part, offrir équitablement leurs services à l'ensemble de la population ; d'autre part, contribuer effectivement à la vie démocratique et à l'exercice de la citoyenneté.

Cette double ambition s'affirme au long du Xx siècle. Les années 1970 marquent le début d'une époque où, plus clairement qu'avant, l'égalité d'accès aux services de la bibliothèque ne semble plus une formule suffisante. Parce qu'elle n'est pas établie encore sur tout le pays ; et parce que l'égalité d'accès n'est pas l'équité d'accès. La distribution uniforme de

145. Citées par Sidney Ditzion en exergue de son livre, ces phrases sont réputées lui avoir inspiré son titre, Arsenals of a Democratic Culture, op. cit. 
l'offre n'en fait pas une offre équitable. D'où le recours à la discrimination positive (affirmative action) : faire plus pour ceux qui sont le plus éloignés des services de la bibliothèque - même s'ils sont proches, physiquement, d'une bibliothèque ${ }^{146}$. Cette politique, disent ses propres promoteurs, demande à être appliquée subtilement car donner plus à certains peut être considéré (est considéré) par les autres comme injuste. Or, dans une démocratie, tout repose sur l'approbation des gouvernés (The Consent of the Governed) : pour ne pas se couper des citoyens, il faut donc assurer à la fois l'égalité d'accès et l'équité d'accès. Desservir la majorité et les minorités. Service for all ${ }^{147}$.

Desservir toute la population, c'est lui proposer des services de bibliothèque adéquats dans tout le pays. La lutte pour le développement des établissements, pour leur financement, pour leur organisation en réseaux, pour la formation des personnels, continue sans relâche. Reste toujours présente à l'esprit l'idée (et la réalité) que, pour obtenir des financements corrects (voire plus), les Public Libraries ont besoin du soutien populaire. Or, les années 1980 inaugurent, sous la présidence Reagan, une ère libérale qui dans tout le pays tendra à diminuer sensiblement l'impôt - et donc les dépenses publiques. Les Américains se voient désormais plus comme contribuables que comme citoyens et donc, dans cette ère de la Tax Revolt, le soutien des usagers est plus que jamais un enjeu stratégique. L'image des bibliothèques, les relations publiques, le lobbying, l'advocacy en faveur des bibliothèques prennent alors une importance encore jamais vue. L'ALA y jouera un rôle majeur.

La nouveauté de cette période est le soin apporté à répondre aux besoins de certaines minorités, phénomène contemporain de leur émergence et de celle de leurs porte-paroles et de leurs revendications dans le champ social. Après la lutte pour les droits civils dans les années 1960, ce sont d'autres minorités qui se constituent, se reconnaissent comme telles, revendiquent comme telles - dans un premier temps les minorités eth-

\footnotetext{
146. «Certains Américains manquent du savoir, des ressources, de l'équipement ou de la formation nécessaires pour participer » (to play the game), Jorge R. Schement. "Imagining Fairness: Equality and Equity of Access in Search of Democracy", Libraries \& Democracy: The Cornerstones of Liberty, sous la dir. de Nancy Kranich. Chicago, ALA, 2001.

147. Bien que traitant de la création d'une bibliothèque universitaire (celle de l'université d'Alabama, en 1830), ces quelques lignes méritent d'être citées : « Le soleil de la science va bientôt se lever sur la région, dardant ses rayons à travers la forêt vers chaque maison et, avec sa chaleur joviale, dissipant les nuages de l'ignorance et des préjugés qui ont trop longtemps assombri le visage de notre pays. Les flots de connaissance qui vont jaillir de cette fontaine du savoir ne devront pas être monopolisés par quelques privilégiés au détriment de la foule. » Cité par F. Ward Hubbs, " Dissipating the Clouds of Ignorance: The First University of Alabama Library », Libraries \& Culture, Winter 1992.
} 
niques et sociales puis, progressivement, les minorités culturelles, sexuelles, religieuses, etc. La Public Library ne doit oublier personne.

L'autre versant de cet investissement démocratique est l'aide, le soutien, les outils que la Public Library propose aux citoyens pour leur participation à la vie démocratique. Dans les Public Libraries, on peut s'inscrire sur les listes électorales ; elles servent de bureaux de vote. Au quotidien, elles permettent l'exercice de la citoyenneté de multiples façons. Il s'agit de rendre disponible l'information gouvernementale (au niveau fédéral comme au niveau des états, des comtés ou des communes), notamment grâce aux sites Web des bibliothèques, qui apparaissent comme un outil très performant pour la diffusion de ce genre d'informations. Dates, ordres du jour, comptes rendus des réunions diverses : le citoyen, s'il ne peut y assister, doit pouvoir joindre les décideurs et faire part de son opinion. On y trouve les informations dont les personnes qui cherchent un emploi ont besoin (annonces, conseils, formation, etc.). On y propose des services dédiés aux immigrants nouvellement arrivés, apprentissage de l'anglais, informations sur la vie aux États-Unis, sur l'école, la santé, le travail, la loi, les transports - la Queens Borough Public Library de New York est réputée dans tout le pays pour son intense activité en ce domaine. On anime aujourd'hui sur le site Web de la bibliothèque, un réseau local (Community Network) en hébergeant les sites locaux publics (la ville, les écoles) comme privés (associations et groupes divers). Cent cinquante ans après la fondation de la Boston Public Library, l'objectif de former des citoyens éclairés est toujours vivace : « Les bibliothèques sont des institutions suprêmement démocratiques. Elles prennent position pour la liberté, l'égalité et les droits de l'homme. L'idée que la démocratie a besoin de citoyens bien informés est peut-être un truisme, mais n'en est pas moins vraie. Plus généralement, la démocratie repose sur l'éducation et les bibliothèques sont partie prenante de l'éducation. Nous devrions toujours nous souvenir des phrases du grand penseur H.G. Wells : "L'histoire humaine devient de plus en plus une course entre l'éducation et la catastrophe." ${ }^{148}$ "

Objectifs intangibles à travers les générations, la formation des citoyens et le soutien à la participation à la vie publique perdurent, avec ces contraintes et ces spécificités nouvelles que sont le cloisonnement de 
l'espace public et la raréfaction des budgets publics. Mais ces difficultés, ces contre-pieds semblent ne motiver que davantage le monde des bibliothèques, à lire Michael Gorman : "Nous croyons en un bien commun croyance en grand désaccord avec la pensée sociale et politique dominante aujourd'hui en Amérique. Nous croyons dans des valeurs qui réconfortent les sans-pouvoir et défendent les minorités, dans un monde où le profit règne en maître sans rival. Nous nous battons pour un service désintéressé, pour l'égalité d'accès et pour l'intégration, dans un monde d'égoïsme, d'exclusion et de division. »

Si l'emphase de ce discours nous est étrangère, voire exotique, voire incompréhensible, c'est sans doute, au-delà des différences culturelles (le patriotisme naïf, tranquille des Américains, leur fierté), parce que les bibliothèques publiques en France ne sont pas du tout considérées comme jouant un rôle comparable : si on reconnaît qu'elles participent à la lutte contre les exclusions, leur position (éventuelle) de contrepoids à « la pensée sociale et politique dominante » n'est ni reconnue ni même souhaitée. En France, les bibliothèques ne sont pas perçues comme un moyen pour les citoyens de se forger librement leur propre opinion.

Ces objectifs démocratiques de la Public Library sont comme couronnés, magnifiés, depuis quelques années, par une intense activité en faveur de la liberté d’information.

\section{La liberté d'information}

Aujourd'hui, en effet, ce qui frappe particulièrement l'analyste étranger (depuis « l'angle européen », pour reprendre la formule d'Axel Munthe), c'est l'énergie dépensée à propos de la liberté d'information : tant du côté de l'ALA que de chaque bibliothèque, on semble très impliqué dans la lutte contre la censure sous toutes ses formes et, plus généralement, contre toute atteinte à la liberté d'information.

Cette préoccupation n'a pas toujours été celle des bibliothèques américaines, occupées à éduquer, former, formater, " américaniser ». Elle a surgi, radicalement nouvelle, à l'orée de la deuxième guerre, guerre d'idées comme Roosevelt le soulignait. Dans ce cadre, ce ne sont plus les bons livres, les bonnes idées, les bons journaux destinés à former des bons citoyens qui sont à mettre à disposition de la population. Mais « tout le spectre de la connaissance humaine » afin que chacun puisse se forger sa 
propre opinion, contrairement à ce qui se passait dans les pays fascistes que l'Amérique en guerre affrontait alors.

Le Premier Amendement est abondamment cité à l'appui de cette nouvelle doctrine. La bibliothèque doit être neutre et faire entrer dans ses collections tous les points de vue, les opinions, les théories. La bibliothèque (les bibliothécaires et leurs soutiens) doit se battre pour que les opinions même impopulaires, même très minoritaires, même odieuses trouvent leur place à la bibliothèque. " Nous devons défendre de mauvais textes en vertu de bons principes. ${ }^{149}$ " C'est l'esprit du principal texte adopté par l'ALA, le Library Bill of Rights *.

On soupçonne bien qu'un parti aussi radical est difficilement tenu. C'est plus qu'un soupçon à l'heure où le politiquement correct s'ajoute aux foudres des conservateurs, créationnistes, homophobes et intolérants de toute nature pour demander le retrait d'ouvrages des rayons des bibliothèques. Aujourd'hui, la critique du Library Bill of Rights est devenue possible non en ce que ce texte est trop peu ou trop mal appliqué, mais en ce qu'il est angéliquement inadapté. Gordon Baldwin, par exemple, argue que la tolérance a des limites (envers les livres négationnistes ou antisémites ou ceux qui expliquent comment fabriquer des bombes, par exemple) et qu'une nouvelle intolérance menace, en particulier, semble-t-il dire, celle des féministes ${ }^{150}$. Je reviendrai sur ces combats, mais il est nécessaire, dès à présent, de souligner les valeurs qui sont mobilisées : l'égal accès à l'information, la tolérance à toutes les opinions, le respect des opinions minoritaires - l'horreur de la tyrannie de la majorité. Tocqueville, on s'en souvient, avait déjà souligné le poids de la pensée majoritaire, son incitation au conformisme intellectuel, culturel, social " en Amérique, la majorité trace un cercle formidable autour de la pensée », et aussi : « Il n'y a pas de liberté d'esprit en Amérique. L'Inquisition n'a jamais pu empêcher qu'il ne circulât en Espagne des livres contraires à la religion du plus grand nombre. L'empire de la majorité fait mieux aux États-Unis : elle a ôté jusqu'à la pensée d'en publier. On rencontre des

*. Voir en annexe.

149. Paul K. McMasters. "Libraries: Where the First Amendment Lives". Libraries \& Democracy, op. cit.

150. «Est-ce que les bibliothécaires devraient bannir des livres parce qu'ils ne décrivent que des femmes infirmières ou parce qu'ils emploient le pronom masculin pour parler des policiers ou des pompiers ? Si les bibliothèques veulent réellement suivre une politique de lutte contre les stéréotypes sexistes, alors elles ne devraient pas posséder de Bible où Dieu est appelé "il". " (Gordon B. Baldwin. “The Library Bill of Rights: A Critique”. Library Trends, Summer 1996) 
incrédules en Amérique, mais l'incrédulité n'y trouve pour ainsi dire pas d'organe ${ }^{151} »$.

Contre cette tyrannie de la majorité, s'élève la liberté d'information. Liberté pour les minorités d'opinion ou de goût, liberté pour les individus. Une minorité, résume Michael Gorman, peut ne se composer que d'une personne (« des minorités aussi petites qu'une personne »). Toute tentative d'élaborer des politiques documentaires standardisées ne peut qu'aller à l'encontre des droits des minorités : "Examinons l'idée que les collections dans les petites bibliothèques comme dans les petites communautés devraient être encadrées par des community standards - doctrine qui nous a menés aux nombreuses et tristes absurdités recensées dans la liste annuelle de l'ALA des livres censurés ou interdits. Une Public Library dans une petite ville doit servir les habitants de la ville, mais sa politique d'acquisitions devrait-elle être basée sur les opinions de la majorité (comme c'est suggéré dans leurs community standards) et alors prétendre empêcher un jeune usager de la bibliothèque de lire L'attrape-cours ? ${ }^{152}$ »

Ces débats très vifs et très présents sont compliqués, aujourd'hui, de la menace que représente, aux yeux de beaucoup d'Américains, l'accès libre à Internet dans les bibliothèques. Le libre accès à Internet est devenu un combat à lui seul, un apogée des luttes déjà anciennes pour faire respecter la liberté d'information. Un combat difficile à mener, reconnaît Nancy Kranich : " Dans la révolution de l'information qui est en train de changer la façon dont nous travaillons, vivons et apprenons, nous ne pouvons plus être sûrs que les descendants de l'invention de Franklin continueront à offrir refuge à toutes les idées et tous les points de vue du monde. L'abondance de l'accès ne se traduit pas en diversité d'accès. Au contraire, nous avons maintenant accès à toujours plus d'idées semblables, les idées alternatives étant marginalisées par la course au profit, les expédients politiques et les caprices du marché. ${ }^{153}$ »

La lutte anti-terroriste, depuis 2001, apporte, à son tour, de nouvelles questions : la liberté d'information a besoin de la confidentialité. Le droit que la loi USA Patriot Act donne aux services de police de savoir ce que lisent, consultent, regardent les usagers des bibliothèques est une nouvelle atteinte aux libertés contre laquelle les bibliothécaires américains

151. Alexis de Tocqueville. De la démocratie en Amérique, op. cit., I, p. 381-383.

152. Michael Gorman. The Enduring Library, op. cit., p. 143.

153. Nancy C. Kranich. "Libraries, the New Media and the Political Process". Libraries \& Democracy, op. cit. 
se mobilisent. L'ALA est, évidemment, en première ligne dans ce combat. Elle est l'un des moteurs de la Campaign for Reader Privacy, qu'elle mène avec les écrivains, les éditeurs et les libraires américains ${ }^{154}$.

Avoir accès librement à l'information pour forger sa propre opinion de citoyen : depuis plus d'un siècle les Public Libraries œuvrent à rendre cet objectif atteignable, à travers des obstacles toujours nouveaux et toujours importants. Nous verrons que cette responsabilité est loin d'être une simple rhétorique mais représente, au contraire, le principal fondement de l'action de l’American Library Association.

\section{Quel héritage ?}

Dans le socle des valeurs qui guident l'activité des Public Libraries, c'est un héritage composite qui s'est construit au cours des deux derniers siècles. Les deux grands courants en sont l'éducation (des citoyens, des jeunes, des immigrants, etc.) et le libre accès à l'information, dans un pays démocratique où chacun doit se forger son propre jugement. Dans l'un, le choix des documents proposés est guidé par leur valeur formatrice, dans l'autre, tout a sa place dans une bibliothèque - contradiction essentielle. $\grave{A}$ cette contradiction s'ajoute la complexité de concevoir un établissement public dans une société où l'espace public est en voie de fragmentation. Celle de l'égalité d'accès sur tout le territoire dans un domaine où la responsabilité n'est que locale. Celle de la prise en compte des besoins de chaque individu tout en construisant un service collectif. Celle de l'abondance d'information qui menace l'accès même à une libre information.

En guise de synthèse, alors que je reviendrai sur ces différents points au long de cet ouvrage, je laisse la parole à Donald Sager qui conclut ainsi un mini-sondage qu'il réalisa en l'an 2000 auprès de ses collègues bibliothécaires sur le thème : "Selon vous, qu'est-ce qui a eu le plus d'impact sur les bibliothèques au $\mathrm{xx}^{\mathrm{e}}$ siècle? ?. Sans doute chagriné que l'informa-

154. Voici comment l'ALA présente sa participation à cette campagne : "The United States is a constitutional republic - a government of the people, by the people and for the people. Popularly called a «democracy », the United States attains that status only when people have access to information on which to base their decisions. That information is available through our nation's libraries. The Public Library is the ultimate marketplace of ideas. By providing a haven that fosters free inquiry, it allows each of us to participate directly in one of the most important elements of a free democratic society - the open and robust debate among competing ideas. Access to information and reader privacy are not issues of politics - they are fundamentally about the Constitution and the freedoms Americans hold dear. True national security depends on the free flow of information our nation's libraries have always provided." 
tique et Internet l'aient emporté haut la main, il apporte son propre grain de sel et sa propre réponse : " Je crois que ceux qui ont réussi les plus grandes choses pour les bibliothèques au $\mathrm{xx}^{\mathrm{e}}$ siècle, ce sont les nombreux femmes et hommes qui sont entrés dans ce métier avec l'objectif de servir leur communauté. Leur but était de servir chacun - jeunes et vieux, immigrants ou hommes d'affaires, riches et pauvres, quelles que soient leur culture et leur origine ethnique. Ce sont ces femmes et ces hommes qui ont reconnu l'importance du libre accès, de l'utilisation des formats bibliographiques, de l'encouragement à la lecture des jeunes, de la défense de la liberté intellectuelle et du partage des collections. C'est le développement de ces valeurs centrales (core values), forgées pendant ce siècle, à travers deux terribles guerres, le brise-cœur de la Grande Dépression, le mouvement pour les droits civils et des batailles sans nombre contre la censure, qui, je crois, représente le triomphe des bibliothèques publiques au Xx $x^{e}$ siècle. C'est cela qui fournit le socle dont tout va émerger au XXI siècle. ${ }^{155}$ »

Énumération enchantée, héroïsation du groupe professionnel : on retrouvera, côté français, certains de ces travers. Ce qui frappe, côté américain, c'est que cette emphase reçoit un écho du côté des pouvoirs publics comme du côté de la population. Vanter les mérites, le rôle, l'utilité de la Public Library est un exercice auquel se livrent régulièrement le président des États-Unis (qui adresse chaque année un message au congrès de l'ALA), les parlementaires, les élus locaux, les responsables associatifs, les enseignants, les citoyens, etc. ${ }^{156}$

La Library Faith a gagné le pays et la fréquentation importante des Public Libraries est en phase avec les discours positifs tenus sur elles. Nous verrons dans les chapitres suivants que tant cette fréquentation que ces discours sont la résultante d'un important travail de conviction, de communication et d'adaptation réalisé par les bibliothécaires américains. Ils sont également la résultante de la foi en l'éducation : l'acquisition des

155. Donald Sager. "Before Memory Fade: Public Libraries in the Twentieth Century”. Public Libraries, March/April 2000.

156. À titre d'exemple, cet extrait du discours d'ouverture du congrès 2005 de l'ALA, à Chicago, par le sénateur démocrate Barack Obama, aujourd'hui président des États-Unis : «Quand des groupes tentent de censurer les grandes œuvres de la littérature, vous êtes ceux qui remettez en rayons Huckleberry Finn et L'attrape-cœurs, assurant ainsi notre droit à la liberté de pensée et à la liberté d'information. Et depuis que nous avons à nous inquiéter que le gouvernement nous surveille dans les bibliothèques (looking over our shoulders in the library), vous vous êtes levés pour défendre notre droit à la confidentialité. Vous êtes les défenseurs à temps plein des libertés américaines les plus fondamentales et, pour cette raison, vous méritez la profonde gratitude de l'Amérique." 
savoirs, des savoir-faire, le self-improvement ne s'arrêtent pas après l'école. Individus responsables, métaphoriquement " chargés de défricher les forêts ${ }^{157}$ », héritiers des pionniers, les Américains ont besoin des bibliothèques pour continuer à apprendre, tout au long de leur vie.

\section{EN FRANCE}

La naissance de la bibliothèque publique en France est quelquefois attribuée à Naudé, quelquefois au décret de 1803 qui confia les bibliothèques de districts aux communes. On peut raisonnablement avancer que ces deux affirmations sont fausses. On pourrait tout autant faire naître cette histoire en 1910, avec la publication de La librairie publique d'Eugène Morel ou en 1968 avec celle du rapport interministériel, La lecture publique en France. Tout dépend, évidemment, du sens, de l'ampleur, de l'ambition que l'on donne à « Bibliothèque publique ». On l'entendra, ici, selon l'usage, au sens de bibliothèque encyclopédique, ouverte à tous, sans conditions d'accès.

\section{De Naudé aux bibliothèques populaires}

On assigne à Gabriel Naudé, célèbre auteur de l'Advis pour dresser une bibliothèque (1627), un progrès décisif vers la bibliothèque publique pour autant que, bibliothécaire de Mazarin, il ouvrit cette bibliothèque au public. Raccourci qui mène à un abus de langage. En effet, si Naudé a bien ouvert la bibliothèque de son maître, d'autres bibliothèques lettrées étaient alors déjà ouvertes, notamment celle du président de Thou. Et si ces bibliothèques sont ouvertes, ce n'est nullement à la population dans son ensemble, d'ailleurs encore largement analphabète, mais aux savants qui en auraient besoin, aux citoyens de la République des lettres. L'Advis n'est donc pas l'acte fondateur d'une bibliothèque publique encore dans les limbes, mais est d'une part un traité sur le fonctionnement des bibliothèques modernes et « aussi et surtout le traité de la bibliothèque docte ${ }^{158}$ » : la bibliothèque de Naudé "s'organise autour d'un unique principe : réunir tout ce qui est utile à la communauté savante ». Et s'il s'agit d'ouvrir la bibliothèque " au moindre des hommes qui en auraient besoin ", "le 
“moindre des hommes" dont il s'agit ici est d'abord un jeune érudit pauvre ${ }^{159} »-$ Robert Damien montre bien qu'il s'agit d'une étape, celle de la constitution d'une « communion convivialisée d'amateurs lettrés » sur le chemin d'une ouverture universelle qui permettra un « usage public du savoir ${ }^{160}$ ». C'est à cette même tradition, celle de l'ouverture aux membres de la République des Lettres, que l'on peut associer la création (par le don de bibliothèques privées à la collectivité publique) des bibliothèques d'Aixen-Provence ${ }^{161}$, Carpentras ou Grenoble.

L'élément fondateur de la bibliothèque publique, au sens où nous l'entendons aujourd'hui, est plutôt le mouvement des Lumières. " La lecture (et le lecteur qui émerge de cette pratique savante) est bien la matrice du grand dessein des Lumières : former le citoyen, par l'instruction. ${ }^{162}$ » La bibliothèque est, dit Robert Damien, l'élément décisif de cette nouvelle conception du savoir liée à l'exercice du pouvoir. On pourrait « montrer, à travers l'histoire des bibliothèques françaises, la maturation progressive de cet idéal qui se réalise peu à peu », à travers trois éléments « discriminants et conjugués qui, interactivement, déterminent la réalisation : passage d'une gestion privée pour un repos savant et divertissant à une gestion publique d'État, socialisante et utilitaire ; élaboration d'une classification encyclopédique qui tend à éliminer l'axe religieux des manuscrits monastiques, au profit des imprimés diversifiés et abondants ; ouverture publique du savoir humain à une population tendanciellement élargie par cette diffusion même. ${ }^{163}$ »

Las, on sait que cette ambition pré-démocratique a été battue en brèche par la soudaineté de la constitution des collections, par l'amoncellement des saisies révolutionnaires. Le rêve de l'Abbé Grégoire - " Ces bibliothèques de parade qui étaient réservées à l'usage de quelque individu, devenues désormais la propriété commune seront accessibles au génie malheureux. L'instruction publique est le creuset qui doit faire distinguer la science de la sottise ${ }^{164}$ » - ce rêve s'enlisera dans les millions d'ouvrages dépareillés, non recensés, voire porteurs d'obscurantisme (les ouvrages de droit canon, les sermonnaires, les bréviaires, les rituels, les

159. Ibid., p. XXI.

160. Robert Damien. Bibliothèque et État, op. cit., p. 57 et 32.

161. Le marquis de Méjanes lègue sa bibliothèque à la ville d'Aix « sous la condition d'en tenir une bibliothèque ouverte en la ville d'Aix pour l'avantage du public auquel elle sera destinée ».

162. Robert Damien. Le conseiller du Prince de Machiavel à nos jours, op. cit., p. 197.

163. Ibid., p. 186.

164. Rapport sur la bibliographie, 1794, cité par Robert Damien, ibid., p. 221. 
almanachs, etc.). Les saisies de 1792 et 1793 n’ont trouvé ni institution ni expertise capables de les mettre à disposition des citoyens : les fonds saisis dans un indescriptible désordre ne sont pas devenus des bibliothèques, collections raisonnées et organisées ${ }^{165}$. Jean Hassenforder résume ainsi l'argument explicatif, aux yeux de beaucoup, du retard français en matière de bibliothèques publiques : «En France, le droit a précédé le fait. Les exigences révolutionnaires ont proposé un modèle idéal. Le pouvoir central en a imposé la réalisation. Mais l'initiative locale n'a pas suivi et les vieilles bibliothèques municipales françaises se révéleront de fait, durant la seconde moitié du siècle, un handicap certain pour la réalisation d'un réseau de bibliothèques publiques modernes. ${ }^{166}$ »

Confiées (« abandonnées » serait plus juste) aux communes en 1803, ces nouvelles collections publiques n'ont pu « devenir l'école de tous les citoyens ${ }^{167}$ ». Cette ambition politique demeure cependant un des substrats de la bibliothèque publique ${ }^{168}$.

\section{Tentative de généalogie de la bibliothèque publique}

Si les Lumières sont le tronc de cet arbre généalogique qui porte les objectifs et les valeurs de la bibliothèque publique jusqu'à nos jours, les modernistes du début du xx siècle, les Morel, les Coyecque, les Sustrac, peuvent en être considérés comme la première branche.

Figure incontournable de l'histoire des bibliothèques publiques, Eugène Morel en est le porte-parole le plus brillant. On a assez souligné l'énergie, l'enthousiasme, la vigueur de son engagement envers ce qu'il appelait «Librairie publique » (Public Library) pour bien marquer la différence avec ce que l'on connaissait alors, en France, sous le nom de bibliothèque : « Notre mot de bibliothèque pue l'allemand. Si l'on y ajoute le mot municipal, l'épouvantail est complet. Non. Il y a pire. Il y a le mot "populaire".

165. En 1847, un rapport au ministre de l'Instruction publique stigmatise « un amas de livres dépareillés qui ne sera pas plus une bibliothèque qu'un amas de briques n'est un palais » (cité dans Le patrimoine : histoire, pratiques et perspectives, sous la dir. de Jean-Paul Oddos, Éditions du Cercle de la Librairie, 1997)

166. Jean Hassenforder. Développement comparé des bibliothèques publiques en France, en GrandeBretagne et aux États-Unis, op. cit, p. 38.

167. Jean-Michel Coupé. Rapport sur les bibliothèques nationales, 1794.

168. On en trouve plus qu'une réminiscence dans la charte adoptée par le Conseil supérieur des bibliothèques en 1991: «La bibliothèque est un service public nécessaire à l'exercice de la démocratie. Elle doit assurer l'égalité d'accès à la lecture et aux sources documentaires, pour permettre l'indépendance intellectuelle de chaque individu et contribuer au progrès de la société. » (Charte des bibliothèques, article 3) 
Théâtre populaire, université populaire, restaurant populaire, bibliothèque populaire... C'est avec ce mot-là que les meilleures intentions dressent de suite une insulte devant l'œuvre qu'elles entreprennent. ${ }^{169}$ » Ouvrir la bibliothèque à tous, sans dédain et sans populisme : c'est le trait principal du modèle de la " bibliothèque publique ", tel qu'il va se constituer tout au long de six ou sept décennies.

La «bibliothèque publique » s'est, en effet, construite contre les deux modèles existant en France à la fin du XIX siècle : les bibliothèques savantes d'une part, les bibliothèques populaires, d'autre part. Les premières, bibliothèques municipales consacrées, institutionnelles, patrimoniales, pour une bonne part noyées sous l'avalanche des collections confisquées un siècle plus tôt, sont de facto peu accessibles ${ }^{170}$. Elles sont centrées sur les collections et portent un intérêt mineur à leurs usagers, réels ou potentiels. " Un immense travail accapare les bibliothèques, on catalogue, on en dresse l'inventaire minutieux. Elles revivent tout leur passé, glorieux certes. Elles sauvent nos trésors... Mais elles chassent les lecteurs, mais elles ne suivent en rien le mouvement de la vie [...]. Pas un instant il ne semble que les bibliothèques sont faites pour qu'on y lise. Le bibliothécaire va là où il y a des livres, des livres qu'on ne lit plus, à classer, à décrire. Et il fuit, là où il y aurait lecteurs. Besogne vulgaire, rejetée dédaigneusement sous le nom de populaire. ${ }^{171}$ » Même si, pour les besoins de la démonstration, Eugène Morel force le trait, il semble bien que le prêt aux usagers ait été souvent réservé aux enseignants ${ }^{172}$. Il est en tout cas avéré que la fréquentation était très faible : 10 lecteurs par jour à Soissons, 22 à Reims, 9 à Bourges, 17 à Orléans, 28 à Grenoble dans les années 1880, selon les décomptes de Graham Barnett.

169. Eugène Morel. La librairie publique. Armand Colin, 1910, p. 179.

170. Comme le résume cette intervention du conseiller municipal socialiste dijonnais, Parizot, en 1901 : « Le coût de cet établissement est beaucoup trop élevé par rapport aux services qu'il rend à l'ensemble de la population [...] Les subventions municipales ne peuvent servir à constituer des collections de bréviaires, d'ouvrages historiques et généalogiques, même très rares, des calembredaines des moines de Cîteaux [...] La bibliothèque n'est pas seulement à l'usage de quelques chercheurs ayant le loisir d'étudier mais elle appartient au peuple, je demande qu'elle soit accessible à tous. » (cité par Olivier Tacheau, « Bibliothèques municipales et genèse des politiques culturelles au XIXe siècle : Dijon et Besançon entre 1816 et 1914 », Bulletin des bibliothèques de France, t. $\left.40, n^{\circ} 4,1995\right)$

171. Eugène Morel. Bibliothèques. Mercure de France, 1909.

172. Graham Barnett indique que c'était, par exemple, le cas des bibliothèques municipales d'Aix, Brest, Nancy ou Valenciennes dans les années 1870 ou 1880. La situation était évidemment variable en raison des conceptions des bibliothécaires : en 1887, le nouveau bibliothécaire de Carcassonne libéralise le prêt, jusque-là réservé aux seuls professeurs ; inversement, en 1885, le nouveau bibliothécaire de Mâcon cesse tout prêt de livres, en raison du désordre qu'il provoque dans les collections. (Graham Barnett, Histoire des bibliothèques publiques en France de la Révolution à 1939. Promodis-Éditions du Cercle de la Librairie, 1987, p. 172-173) 
L'autre type de bibliothèque, les bibliothèques populaires, est un type composite. Créées et/ou soutenues par des militants de l'éducation populaire (la Société Franklin, la Ligue de l'Enseignement), par le patronat chrétien, par des syndicats, par des municipalités ou par de simples associations, elles se développent jusqu'au début du Xxe siècle, où on en compte environ 3 000, mais rencontrent des critiques croissantes de la part de leurs usagers, concernant «l'insuffisance des locaux, la non-qualification du personnel, les heures d'ouverture limitées et, par-dessus tout, les fonds devenus délabrés, inintéressants et périmés. Ces défauts provenaient, directement ou indirectement, du manque de moyens ", analyse Graham Barnett. C'est que leur statut, indépendant, fait que leur financement n'est pas assuré. Leur personnel est souvent bénévole, les heures d'ouverture très limitées (souvent seulement le dimanche après-midi) et les crédits d'acquisition sont très faibles. Bref, ces bibliothèques ne rendent pas les services que l'on attend d'elles et ne peuvent être considérées que comme des repoussoirs par les promoteurs de la bibliothèque publique.

Contre les "musées de livres » et contre les institutions charitables, "le temps est venu, après un demi-siècle d'efforts qui triomphent aujourd'hui en Angleterre, en Amérique, de concevoir la lecture comme un service public, municipal, analogue à la voirie, aux hôpitaux, à la lumière. ${ }^{173}$ » Cette bibliothèque publique, ces bibliothèques publiques, « rajeunies et transformées pourraient enfin remplir pleinement le grand rôle d'éducateur qui leur est assigné dans une démocratie et devenir partout, suivant la forte expression américaine, The University of People, l'Université du peuple », écrivait en 1904 le bibliothécaire Jules Laude ${ }^{174}$. Modèle et vocabulaire américains, anglo-saxons, parfaitement assumés aussi par les " néo-modernistes » des années 1960 : « Il s'agissait [dans les années 1960] de définir la mission de la bibliothèque publique au sens anglo-saxon de l'expression. ${ }^{175}{ }$ Ainsi, ouverte à tous, la bibliothèque

173. Eugène Morel. Bibliothèques, op. cit.

174. Jules Laude. "Quelques mots sur les bibliothèques françaises », cité par Graham Barnett, Histoire des bibliothèques publiques en France de la Révolution à 1939, op. cit., p. 215. Il est notable, significatif et regrettable que les réflexions sur les missions des bibliothèques proviennent, en France, presque exclusivement des bibliothécaires eux-mêmes, alors que la classe politique en parle peu. Par indifférence ? C'est ce qu'avance Jean Hassenforder : « La crise des bibliothèques, au début du XX⿳亠丷厂犬⿱ été par ailleurs les intentions affirmées sur le plan politique. Aussi, Eugène Morel, pionnier de la bibliothèque publique en France peut-il écrire : "Les majorités laïques et politiques l'ignorent parfaitement.” » (Jean Hassenforder, Développement comparé des bibliothèques publiques en France, en Grande-Bretagne et aux États-Unis, op. cit., p. 95)

175. Michel Bouvy. "Une revue professionnelle de combat : Médiathèques publiques », Mémoire pour demain, mélanges en l'honneur de Albert Ronsin, Gérard Thirion, Guy Vaucel. ABF, 1995. 
publique a pour ambition d'être un outil de formation et d'information. Eugène Morel l'oppose radicalement aux bibliothèques municipales de son époque : "Considérez comme rien, comme une sorte de panoplie, la belle bibliothèque, son bibliothécaire et ses milliers de bouquins. C'est en fondant ailleurs une librairie utile que vous attirerez la foule, répandrez le goût de lire, les connaissances exactes, le savoir professionnel, révélerez au pays à quoi servent les livres. ${ }^{176}$ " Foin du beau inutile. La bibliothèque, la "librairie publique ", n'a de sens qu'utile à tous.

La bibliothèque publique $\mathrm{a}$, en termes d'éducation comme de culture, un souci de déségrégation : il faut en finir avec la bibliothèque pour les bourgeois et la bibliothèque pour le peuple. Le « modernisme bibliothéconomique », défendu par exemple par Charles Sustrac, premier secrétaire général de l'ABF, "s'inscrit dans une idéologie sociale de progrès qui rejette tout privilège, toute exclusion, toute ségrégation dans l'accès à l'information, au savoir, à la culture ", écrit Noë Richter. Et aussi : "La bibliothèque publique moderne sera fondée en opposition avec la bibliothèque publique traditionnelle et avec la bibliothèque populaire. La première a privilégié l'érudition historique, littéraire et juridique, et les textes classiques. Elle a rejeté la création littéraire vivante, la vulgarisation et les textes écrits pour distraire. Elle s'est sclérosée et transformée en musée du livre mort. La seconde n'a cherché qu'à conditionner une masse réputée dangereuse et inaccessible aux lumières de la raison en lui concédant une information et une lecture soigneusement triées. ${ }^{17}$ "

Ainsi, la bibliothèque publique doit rassembler dans un même projet l'outil que sont les riches collections de la bibliothèque municipale et le souci du lecteur qu'ont les bibliothèques populaires. Il faut casser la dichotomie entre ces deux réseaux, de plus en plus inadaptés car la société évolue, son niveau d'éducation s'élève, ses besoins documentaires, ses pratiques culturelles changent. Tout au long $d u x^{e}$ siècle, des générations successives de bibliothécaires plaident pour un changement radical de la conception et donc de l'utilité de la bibliothèque. Devant le comité interministériel de 1966-1967, Michel Bouvy, alors président de la section lecture publique de l'ABF, est, à son tour, l'ardent promoteur de ce modèle : " À cette conception bi-partite de la bibliothèque municipale, qui me paraît condamnable, s'oppose la conception unitaire, celle qui est adoptée par presque tous les pays étrangers, sinon par tous, celle de la biblio- 
thèque publique. La bibliothèque publique, c'est la bibliothèque unique pour toute la population, prêtant largement ses collections, largement ouverte à tous. Elle est à la fois bibliothèque de distraction et bibliothèque d'étude [...]. L'idée de la bibliothèque pour l'élite et celle de la bibliothèque populaire, ce sont les deux idées qui ont toujours fait le plus de mal aux bibliothèques françaises, et malheureusement ce sont les plus répandues à l'heure actuelle dans la plupart des milieux. La bibliothèque publique n'est, il faut le répéter, ni un service de luxe ni une œuvre de bienfaisance. C'est un service public aussi utile que l'école. »

Mais l'éducation des adultes et, plus généralement, tout ce qui est documentation n'est pas resté au cœur du projet de bibliothèque publique tel qu'il a été importé, en ceci donc adaptation fautive du modèle américain. La mise en œuvre du modèle en France, à partir des années 1960 et 1970, a d'abord, surtout, été une rupture avec la bibliothèque savante. La disparition des "sections d'étude ", silencieuse et générale, en est un signe manifeste. Conserver cette organisation (section de prêt/section d'étude), analyse-t-on, serait perpétuer la hiérarchie entre deux services, deux collections et deux publics. Il faut donc décloisonner. Les reproches que la Direction du livre adresse au projet de la médiathèque de Nantes, en 1979, sont clairs : "Cette nouvelle prise de parti semble difficilement défendable dans la mesure où elle entérine l'opposition traditionnelle entre lecture savante et lecture populaire et où elle limite le prêt aux besoins les plus immédiats. ${ }^{178}$ "

Cette adaptation infidèle du modèle anglo-saxon est dénoncée très tôt, dès 1969, par Albert Ronsin, l'un des bibliothécaires modernistes : « Il semble qu'il y ait un malentendu sur la question des "Bibliothèques publiques”. Nous les voyons, en France, sections de prêt ; en réalité, dans l'optique internationale, et c'est ce que M. Bouvy avait vu lorsqu'il avait créé la section, c'est la bibliothèque publique style allemand ou anglosaxon avec livres précieux, collections d'étude de plusieurs millions de livres. Il est vrai que jusqu'alors seul le problème du grand public et du prêt a retenu l'attention. Les fonds anciens et d'érudition ont été si longtemps le seul souci, que tout était à faire en lecture publique. ${ }^{179}$ »

Michel Bouvy revient à plusieurs reprises sur ce désaccord conceptuel. Il oppose ainsi la « lecture publique » (qu'il nomme toujours entre

178. Cité dans Anne-Marie Bertrand, Les villes et leurs bibliothèques, op. cit., p. 100.

179. Lettre à Pierre Gras, 16 janvier 1969. Cité dans Anne-Marie Bertrand, Les villes et leurs bibliothèques, op. cit., p. 158. 
guillemets), pour lui vision erronée et populiste, et la « bibliothèque publique », qui a ses faveurs. Sur les collections : « Le rôle principal de la bibliothèque publique n'est pas de prêter des livres de poche (cela, c'est la "lecture publique", au sens le plus péjoratif de l'expression), mais de prêter plutôt des livres que le lecteur ne pourrait s'acheter. ${ }^{180}$ "Sur le concept lui-même (à propos de la jeune BPI) : « C'est là qu'on voit combien la notion de bibliothèque publique a été mal digérée par les promoteurs, obnubilés par celle de "lecture publique" qui prévaut, hélas, encore dans notre pays. Je m'explique. On a appliqué à une grande quantité de documents de tout niveau des principes qui sont valables à un niveau moins élevé. La notion d'ouvrages de référence n'a pas été comprise [...]. Je conçois parfaitement que certains collègues ne soient pas de mon avis, et en particulier les tenants de la "lecture publique" qui verront dans cette bibliothèque plus qu'autre chose un symbole, qui feront plus attention à l'ouverture apparente qu'à l'activité réelle et à la qualité du service rendu sous tous ses aspects, et qui oublieront trop facilement l'importance pour une ville comme Paris, comme pour toute la France, de la notion de réseau avec des unités aux caractéristiques bien étudiées. ${ }^{181}$ »

Albert Ronsin, plus tardivement, évoque à nouveau « la transformation de la notion de lecture publique en celle de bibliothèque publique et ce ne fut pas une simple question de vocabulaire : il s'agissait rien moins que de substituer à la notion d'établissement de distraction et d'information celle d'établissement culturel de documentation au service de la population tout entière. ${ }^{182}$ "

Ainsi, si c'est le modèle anglo-saxon qui est promu depuis le début du siècle et qui sera adopté dans les années 1960, il ne l'est pas dans son intégralité - dans son intégrité. Il est adopté dans certains de ses objectifs (et dans certains seulement - notamment pas dans le registre éducatif) mais pas dans son substrat politique. L'idée que la nation a besoin de bibliothèques pour éduquer ses citoyens est peu présente ; l'ouverture à tous est vue comme la fin d'un privilège archaïque plutôt que comme une révolution culturelle ; la notion de réseau a disparu ; la responsabilité des pouvoirs locaux est absente tout comme l'intervention de la population ellemême ; l'État, enfin, est supposé y jouer un rôle moteur, ce qui n'est évidemment pas le cas aux États-Unis. 


\section{L'État-providence}

Après la seconde guerre mondiale, l'Europe occidentale connaît l'extension des prérogatives de l'État. Politique économique, politique industrielle, politique sociale, politique éducative, politique de santé publique, politique culturelle... : le champ où l'intervention de l'État est légitime est devenu immense.

Dans ce continent épuisé par deux guerres atroces, il s'agit de reconstruire le pays mais surtout de le moderniser dans un souci de justice sociale. L'État est (redevenu) l'instituteur de la société. « L'impératif de modernisation ", dit Pierre Rosanvallon, «s'appuie sur la dénonciation des retards et des archaïsmes de la société française [...]. La génération de hauts fonctionnaires qui émerge après la Libération se sent d'une certaine façon au-dessus de la société, jugée retardataire, archaïque, dans une position de guide et de pédagogue ${ }^{183}$ ». L'État est chargé de la reconstruction (économique) et de la redistribution (sociale). Cette ambition, en France, l'État «paternaliste-pédagogue » (Pierre Rosanvallon) est le seul à avoir les capacités de la mener à bien. Les collectivités territoriales ne sont pas encore de véritables acteurs politiques et jouent « les belles endormies ». Jusque dans les années 1970, « en pleine période de croissance, les collectivités locales étaient considérées comme archaïques, freins au progrès et à l'expansion, agents supplétifs de la modernisation du pays loin derrière l'État », rappelle Dominique Lorrain ${ }^{184}$.

C'est pourquoi pendant plus de trente ans, c'est de l'État que l'on attendra le développement, la modernisation, la démocratisation des bibliothèques publiques ${ }^{185}$. Ainsi, Pierre Lelièvre, en 1944 : « La lecture publique doit être un service public, mais un service où l'État comprend et assume sans réserve, comme sans arrière-pensée, un rôle d'éducateur. ${ }^{186}$ » Pierre Gras, longtemps bibliothécaire de Dijon, est l'un des plus ardents promoteurs de l'intervention de l'État. En 1968, il demande même une intervention radicale car il est, écrit-il, «indispensable de "nationaliser"

183. Pierre Rosanvallon. L'État en France de 1789 à nos jours. Éditions du Seuil, 1990 (L'univers historique).

184. Dominique Lorrain. « De l'administration républicaine au gouvernement urbain ». Sociologie $d u$ travail, $\mathrm{n}^{\circ} 4,1991$.

185. Cette croyance excède, évidemment, le monde culturel et, a fortiori, celui des bibliothèques. Pour les musées, Dominique Poulot parle de « cette unique légitimité qui appartient à l'État national - loi d'airain, culturelle et politique, qui surplombe l'histoire française contemporaine ». (Une histoire des musées de France, XVIII ${ }^{\circ} X X^{e}$ siècle. La Découverte, 2005, p. 6)

186. Pierre Lelièvre. Projet d'« organisation d'un service de la lecture publique » (22 septembre 1944) 
complètement les bibliothèques municipales et, au moins, leur personnel. C'est ce que l'État a été amené à faire pour l'enseignement primaire. ${ }^{187}$ " Alors que la Direction ministérielle « veut faire aveuglément confiance aux municipalités », il proteste contre cette cécité, tout particulièrement, « à Dijon, où la Ville ne veut pas développer les bibliothèques ${ }^{188}$ ".

On verra longtemps, notamment dans les travaux de l'ABF, la croyance que l'intérêt général n'est compris que de l'État et que l'on ne peut confier aux villes la construction d'un service public de la lecture. Ceci à la fois en raison de l'émiettement communal en France (qui rend l'organisation raisonnée du territoire très difficile) mais aussi en raison des responsabilités propres à l'État. Comme le stipule le préambule de la Constitution, " la Nation garantit l'égal accès de l'adulte et de l'enfant à la formation et à la culture ». Il appartient à l'État, disent les bibliothécaires, de garantir cette égalité d'accès - notamment par une loi sur les bibliothèques et par une direction d'administration centrale spécifiquement dédiée à ce secteur, deux gestes éminemment symboliques de l'intérêt porté par l'État aux bibliothèques. Si l'adoption d'une loi sur les bibliothèques a toujours été repoussée ou évitée, nous y reviendrons, la Direction des bibliothèques et de la lecture publique (DBLP), créée en 1944, est un des premiers enfants de l'État-providence. Elle est la manifestation de la volonté et de la capacité à prendre en mains le destin des bibliothèques. Mais, on le sait, la conjoncture économique et sociale (et, en particulier, le baby-boom et l'explosion du nombre d'enfants dans le système scolaire puis de jeunes dans le système universitaire) a conduit la DBLP à s'occuper prioritairement des bibliothèques universitaires. Bibliothèques sur lesquelles, de surcroît, elle avait pouvoir de décision, ce qui n'était pas le cas pour les bibliothèques municipales, dépendantes des communes ${ }^{189}$.

De fait, ce n'est au mieux que dans les années 1970 que la notion même d'un service public communal sera tout à fait légitimée. Encore aujourd'hui, reste vivace l'idée que l'État joue un rôle tutélaire pour les bibliothèques publiques, même si les moyens de son intervention ont évolué, désormais davantage tournés vers des programmes contractuels ciblés sur des objets précis et des périodes limitées ${ }^{190}$ que vers l'organisation équitable du ser-

187. Pierre Gras. « Pour la nationalisation des bibliothèques municipales ». Bulletin d'informations de l'ABF, $\mathrm{n}^{\circ}$ 58, 1968.

188. Lettre du 11 février 1970. Cité dans Anne-Marie Bertrand, Les villes et leurs bibliothèques, op. cit., p. 95.

189. Anne-Marie Bertrand, Les villes et leurs bibliothèques, op. cit., p. 54-56.

190. Les bibliothèques municipales à vocation régionale, les contrats ville-lecture, les « ruches », etc. 
vice public de la lecture sur le territoire. On constate ainsi que, surtout depuis les lois de décentralisation des années 1980, la logique de liberté et de responsabilité des collectivités territoriales l'emporte sur la logique d'aménagement du territoire.

$\mathrm{Au}$-delà des faits, demeure cependant toujours une demande d'État comme juge de paix et référent ${ }^{191}$. Demeure aussi, dans cet héritage de l'État-providence, l'idée de partage (la " démocratisation culturelle ») et l'idée d'intérêt général mis en œuvre par un service public. Le privé, les bénévoles sont vus comme hors de propos, comme des intrusions sans légitimité. Né dans le giron de l'État, le concept de bibliothèque publique exige un service public.

\section{La démocratisation}

En 1948, le rapport La lecture publique en France déplore : "Notre pays, qui est très certainement un de ceux qui possèdent les bibliothèques les plus riches du monde, est donc aussi un de ceux où le livre est le plus inaccessible à la masse [...]. En 1945, la grande majorité de nos bibliothèques municipales de province sont ou des bibliothèques de conservation et d'étude, ou de simples cabinets de lecture, refuge d'érudits et de retraités de l'enseignement. Le grand public n'y vient pour ainsi dire pas, ignorant même parfois jusqu'à leur existence. »

Tous les acteurs, les analystes, les praticiens l'ont dit dès les années 1940. Au milieu du xx ${ }^{e}$ siècle, la France ne manque pas de bibliothèques (publiques), elle en a au contraire un très grand nombre, qu'elles soient municipales, populaires, paroissiales, associatives, etc. Mais ces bibliothèques ne sont en rien adaptées aux besoins de la population. La bibliothèque publique, on l'a vu, est l'outil qui va permettre de répondre à ces besoins. Augmenter, améliorer l'offre de bibliothèque, c'est le moyen de répondre à ces besoins.

Las, au début des années 1980, ce credo qui avait traversé tout le siècle sans encombres est soudain battu en brèche. Non, ce modèle de bibliothèque publique n'est pas le moyen d'assurer la démocratisation de l'accès au livre et à la documentation. S'il a augmenté nettement le nombre des usagers ${ }^{192}$, il le fait sur des bases à la fois erronées et insuffisamment

191. Un des derniers exemples en est la réponse aux agissements des municipalités du Front national dans les bibliothèques de leurs communes, entre 1995 et 2001.

192. De 914000 usagers inscrits en 1969 à 3394000 en 1983. 
ambitieuses ${ }^{193}$. Ces bibliothèques peuvent bien être publiques, elles ne sont pas populaires.

Au-delà du diagnostic, deux séries de critiques, d'ailleurs complémentaires, sont avancées : d'une part, les bibliothèques sont faites par les classes moyennes pour les classes moyennes ; d'autre part, c'est le côté patrimonial, savant, qui rebute les classes populaires.

Ce sont les sociologues, notamment Jean-Claude Passeron et Nicole Robine, qui les premiers mettent en avant le caractère discriminatoire de la fréquentation des bibliothèques. Par leur offre, leur organisation, leurs choix, les bibliothèques municipales sont conçues pour les classes moyennes, voire pour les lettrés. Jean-Claude Passeron : « Il est vain de croire que l'augmentation de l'offre de la lecture en bibliothèque produira mécaniquement, en même temps que l'augmentation globale de l'intensité de lecture, l'augmentation de la part des forts lecteurs dans les classes populaires. Par ses techniques d'offre comme par les ouvrages qu'elle offre, la bibliothèque correspond d'abord aux attentes et aux pratiques culturelles des classes moyennes. ${ }^{194}$ » Nicole Robine : « Tout ce que les classes favorisées valorisent dans une bibliothèque ou une librairie : l'éclectisme, la variété des choix dans un même genre, le mode de classement des ouvrages [représentent pour les petits lecteurs] des facteurs d'éloignement de ces institutions dont l'agencement est conçu pour des lettrés, par des lettrés. ${ }^{195}{ }^{»}$

Cette analyse sociologique est rapidement intériorisée par les bibliothécaires, qui acquiescent au soupçon qui pèse sur la bibliothèque et dès 1988, Le métier de bibliothécaire, publié par l'ABF, vulgarise ces analyses : "Pendant longtemps on a cru que la simple ouverture de bibliothèques suffirait à gagner de nouveaux lecteurs, que la gratuité du livre en assurerait la démocratisation, et qu'ainsi viendraient progressivement à la lecture des individus et des couches sociales qui auparavant ne lisaient pas. À l'usage, il n'en est rien : 14,3\% seulement de Français de plus de 15 ans sont inscrits en bibliothèques, tous types de bibliothèques publiques confon-

193. On se souvient de l'interpellation célèbre de Gérald Grunberg, alors président de la section des bibliothèques publiques de l'ABF : « Nous ne pouvons pas parler de bibliothèque en la définissant simplement comme un équipement qui prête des livres à 20 ou $30 \%$ de la population, ce qui suppose que 70 à $80 \%$ restent à l'écart et que cela regroupe, le plus souvent, les catégorie sociales les plus défavorisées. La bibliothèque draine un public qui socio-culturellement est prédisposé à venir la fréquenter, mais il reste alors une immense majorité du public que l'on ne touche pas. » Intervention au colloque d'Hénin-Beaumont. Lecture et bibliothèques publiques. ORCEP, 1982.

194. Jean-Claude Passeron. "Le polymorphisme culturel de la lecture », 1981, repris dans Le raisonnement sociologique. Nathan, 1991, p. 339.

195. Nicole Robine. Les jeunes travailleurs et la lecture. La Documentation française, 1984. 
dus (13,2 \% en 1973), et parmi ces inscrits se retrouve l’inégale répartition des lecteurs. Fréquentent d'abord les bibliothèques les habitués de la lecture : cadres supérieurs et moyens, élèves et étudiants, bacheliers et diplômés de l'enseignement supérieur, parisiens et habitants des grandes villes sont les fidèles adhérents. Les travaux de Jean-Claude Passeron et de ses élèves dans les bibliothèques de la région parisienne, ceux de Jean-François Barbier-Bouvet et Martine Poulain à la BPI, rappellent que par ses techniques d'offre comme par ses contenus offerts, la bibliothèque correspond surtout aux attentes et aux pratiques culturelles des classes moyennes. » (p. 261-262)

Ce discours autocritique des bibliothécaires stigmatise jusqu'aux bébés des classes moyennes, comme le montrent ces deux citations à propos de l'accueil des bébés-lecteurs : « On a dévoyé cette action. Le but d'ACCES, c'était de toucher des milieux défavorisés qui étaient loin du livre et de la culture. » Ou bien : "ACCES travaillait avec les milieux défavorisés. Ce travail a été détourné par les bibliothécaires. On accueille à tour de bras des crèches, on fait de l'éveil à des enfants déjà acquis. Ce n'est pas la même chose que d'aller en PMI. Trop de bibliothécaires ne savent plus pourquoi on travaille avec des tout-petits. ${ }^{196}$ »

La deuxième critique avancée contre le modèle de bibliothèque mis en œuvre est qu'il est trop proche de l'ancien modèle de bibliothèque savante. Ce qui plombe le développement des bibliothèques, analyse-t-on, c'est l'étude, c'est le patrimoine. Analyse courante qui conduit à déplorer la présence massive des étudiants ou le poids du patrimoine et qui, on l'a vu, est en rupture avec le modèle importé d'Amérique. Dans son analyse des publics des services patrimoniaux des bibliothèques, Florence Belot souligne le peu de poids symbolique qui leur est reconnu : "Certes, il ne doit pas y avoir de bibliothèques publiques pour les lettrés virtuoses (les bibliothèques d'étude) et d'autres pour les moins habiles lecteurs (les bibliothèques de prêt), mais il n'est pas absurde de se demander si les bibliothèques modernisées n'ont pas - en voulant casser cette dichotomie - de fait, marginalisé, délégitimé l'activité de recherche en leur sein. ${ }^{197}$ ». L'image de la bibliothèque savante, forcément poussiéreuse et élitiste, sert de

196. Cité par Jean-Claude Utard, « Les préoccupations des bibliothécaires jeunesse par rapport à leur métier », communication au colloque du 6 juin 2002 organisé par la Joie par les livres (compte rendu dans le Bulletin des bibliothèques de France, t. 47, $\mathrm{n}^{\circ} 5,2002$, « Les bibliothèques pour la jeunesse et leurs publics »).

197. Florence Belot. Le public des fonds anciens des bibliothèques municipales : silences et représentations. Mémoire d'étude du diplôme de conservateur des bibliothèques : enssib, 2004. Les formules en italique sont de Jean Gattégno. 
repoussoir à la conception moderne des bibliothèques - Joëlle Le Marec le souligne à son tour : "Les institutions culturelles, en particulier musées et bibliothèques, sont aujourd'hui le théâtre d'un phénomène majeur qui leur est commun... Le fameux musée poussiéreux, refuge des dimanches pluvieux, la bibliothèque hantée par quelques érudits silencieux environnés de gros volumes spécialisés sont désormais des figures repoussoirs que s'évertuent à conjurer les établissements engagés dans des rénovations et des restructurations considérables. ${ }^{198}$ "

À la fin du xx siècle, cette double critique adressée à la bibliothèque (n'attirer que les classes moyennes, être une bibliothèque savante) revient à faire le constat de l'échec du modèle de bibliothèque publique, destiné à attirer l'ensemble de la population, tous besoins confondus, de la formation aux loisirs (alors même que le modèle " adapté » qui est mis en œuvre est largement amputé de son ambition formatrice). Derrière ce constat, se dresse, en ombre chinoise, la présence insistante de la bibliothèque patrimoniale, la bibliothèque d'autrefois. Le modèle de bibliothèque publique, disent les analystes, ne fait que prolonger le modèle honni d'autrefois. Une autre politique est à inventer qui tiendra compte des insuffisances de la bibliothèque. C'est donc logiquement que, à partir de 1981, les nouveaux pouvoirs publics, structurellement réticents aux dispositifs institutionnels et conjoncturellement convaincus par une petite avant-garde de sociologues et de bibliothécaires, tiendront un discours offensif contre la bibliothèque (comme institution) et pour la diversification des publics.

Ce discours de rupture se retrouve, au plus haut niveau, dans le plan intérimaire (1982-1983), avec le rapport du groupe culture, L'impératif culturel, publié en novembre $1982{ }^{199}$ : « Nous n'avons pas voulu réduire le culturel à la création, la diffusion ou la conservation d'œuvres ou de pratiques séparées du quotidien selon des critères issus finalement d'une conception idéologique de la "culture cultivée" ou de normes institutionnelles. » La démocratisation mise en œuvre par l'État ne fonctionne pas et, d'ailleurs, est un objectif non pertinent : " On doit analyser avec un esprit critique la politique de démocratisation de la culture cultivée mise en œuvre par l'État. Une grande partie de cette culture se rattache, en effet, à des arts et des pratiques légitimés dans le passé par des classes

198. Joëlle Le Marec. « Public savant, public profane » in L'action culturelle en bibliothèque, sous la dir. de Viviane Cabannes et Martine Poulain. Éditions du Cercle de la Librairie, 1998, p. 29.

199. La Documentation française. 
sociales dominantes ; en tant que telle, elle mérite tous les soins qui doivent être accordés à un élément essentiel de notre patrimoine. Mais on ne voit guère pourquoi il serait nécessaire d'y acculturer un public qui y reste structurellement rétif [...]. »

En ce qui concerne les bibliothèques publiques, ce nouveau discours est inauguré au colloque d'Hénin-Beaumont, en novembre 1981, un discours de la sociologie critique (par les interventions de Jean-Claude Passeron et Nicole Robine) et un discours politique qui déplace la responsabilité des bibliothécaires vers les élus et les usagers : " J'ai entendu dire », dit Jean Gattégno, directeur du livre, «que, pour beaucoup de professionnels, l'un des risques encourus dans la nouvelle démarche qui est mise en œuvre, dans ce transfert considérable du pouvoir de l'État vers les collectivités locales, vient de ce que les élus locaux sont souvent mal informés, voire peu concernés par le problème de la lecture et de la lecture publique en particulier [...]. Il est probable que nous sommes plus compétents pour parler des bibliothèques que tel ou tel élu. Il n'est pas évident du tout que nous soyons plus compétents pour parler de la lecture. En tout cas, quand bien même ce serait vrai, il me semble que l'un des enjeux de la démarche du gouvernement actuel est précisément de prendre ce risque. Mieux vaut que les élus, et la population qui les a élus, se sentent responsables avec leur "impréparation" plutôt que de perpétuer un système où les choses nous sont dictées d'en haut sous forme de normes indicatives, sous forme de programmes types. ${ }^{200}$ »

Les bibliothèques publiques sont, alors, considérés comme des institutions élitistes (même si le mot n'est pas prononcé) et comme devant changer leurs objectifs. Appuyée sur le rapport Pingaud-Barrault, cette nouvelle politique est baptisée " extension de la lecture » : elle incite les bibliothèques à travailler avec de nouveaux partenaires (les prisons, les hôpitaux, les comités d'entreprise, les quartiers d'habitat social...) et en direction de « nouveaux publics » (jeunes, immigrés, handicapés, pré-retraités...) ${ }^{201}$, puisque la lecture (et, donc, l'extension de la lecture) excède le cadre, l'activité et même les missions de la bibliothèque municipale. Les pratiques de lecture, au contraire, se caractérisent par une «multitude indéfinie des publics » et une «multitude indéfinie des lieux ${ }^{202}$ » d'usage. Le 25 janvier 1984, dans une communication au Conseil des ministres, Jack

200. Actes du colloque Lecture et bibliothèques publiques. Hénin-Beaumont, 20-21 novembre 1981. ORCEP, 1982.

201. Isabelle Jan. Rapport sur l'extension de la lecture publique. Hôpitaux, prisons, entreprises. DLL, 1983.

202. Anne-Marie Chartier, Jean Hébrard. Discours sur la lecture, Fayard-BPI, 2000. 
Lang réaffirme « sa volonté d'étendre le territoire du livre. Il [s'agit] essentiellement de contribuer à l'effort entrepris par les bibliothèques publiques en direction de nouveaux lieux de lecture et de nouveaux publics. ${ }^{203}$ »

Cette dernière période, on le voit, est donc porteuse de deux ruptures par rapport au modèle de bibliothèque publique. D’une part, elle cesse de considérer le public (ou la population à desservir) comme une unité mais voit, au contraire, en lui une multiplicité de publics-cibles. D'autre part, la fonction éducative et patrimoniale de la bibliothèque est sévèrement critiquée.

Le discours de la démocratisation est, ainsi, devenu indissociablement un discours sur l'échec de la démocratisation.

\section{Quel héritage ?}

Cet héritage qui s'est construit au cours des deux derniers siècles est, on le voit, équivoque. Il est composé de deux courants principaux, que l'on peut considérer comme antagonistes. D'abord, dans la lignée des Lumières, la bibliothèque publique est considérée comme devant former les citoyens et comme contribuant ainsi à l'exercice de la démocratie, qui a besoin de citoyens éclairés. Mais l'héritage révolutionnaire a pris la figure déroutante de la bibliothèque patrimoniale, aristocratique, fermée à la population. Il ne pouvait être assumé dans la société urbanisée et éduquée du $\mathrm{xx}^{\mathrm{e}}$ siècle. L'ouverture du trésor à tous a signifié la fin de la bibliothèque savante (au double sens de celle qui sait et qui enseigne).

Cette sourde lutte (non dite et non écrite) contre ses propres racines met les bibliothécaires en porte-à-faux avec eux-mêmes. S'ils continuent à se revendiquer des Lumières, ils nient de fait la formation comme un des objectifs de la bibliothèque publique. Les humanités, le patrimoine, la recherche sont considérées comme déplacés car ayant l'effet pervers d'attirer à la bibliothèque ses habituels usagers, les étudiants et les classes moyennes. Ils sont accusés de ramener la bibliothèque vers ses errements passés, d'empêcher l'évolution de la bibliothèque en une institution réellement démocratique. Le partage du savoir est ainsi, de facto, exclu de l'ambition démocratique.

Le second héritage est celui de la bibliothèque publique, importation 
de la Public Library. Mais, on l'a vu, importation infidèle, faussée justement par le refus de la bibliothèque savante. Cette « bibliothèque publique » à la mode française a pris le visage des médiathèques : bibliothèques modernes, ouvertes, démocratiques mais dont le substrat politique a disparu. Les caractéristiques techniques, notamment l'introduction de nouveaux médias, ont pris le pas sur le projet politique. La modernité (la modernisation) est devenue le maître mot du développement des bibliothèques en France depuis une trentaine d'années - à commencer par la modernisation de leur nom, " médiathèques », mot intraduisible.

Le premier héritage (la bibliothèque savante) est refusé. Le second (la bibliothèque publique) est assumé mais de façon fautive. Les conséquences pour les bibliothèques en sont forcément importantes. D'abord, une image confuse de la bibliothèque et, donc, une difficile mobilisation autour d'elle. Il était plus facile, constate Jean Hassenforder, de créer des bibliothèques (aux États-Unis) à partir de rien que d'essayer de développer des bibliothèques publiques à partir des " cimetières de livres » (Eugène Morel) que sont accusées d'être les bibliothèques municipales du XIX siècle. Mais la table n'est pas rase. Au contraire : même dans la position de rupture qui est celle des tenants de la lecture publique, on trouve encore nombre de survivances et de caractères acquis : la création des médiathèques, à la fin $\mathrm{du} \mathrm{xx}^{\mathrm{e}}$ siècle, a en réalité repris bien des figures des bibliothèques savantes tant décriées - par exemple, la primauté des collections littéraires sur les documents pratiques, une faible place faite à l'actualité, une méfiance profonde vis-à-vis du politique, une culture de service bien souvent défaillante, la faiblesse de l'implication locale.

La volonté farouche de se démarquer du modèle ancien se traduit plus dans le discours que dans la réalité. Mais, deuxième conséquence, le discours sur la bibliothèque et son utilité sociale met l'accent sur le type de population touché. Est ainsi aujourd'hui considérée comme démocratique la bibliothèque qui accueille non pas l'ensemble de la population mais la part de la population qui en était auparavant exclue. D'où cette valorisation permanente du travail accompli vers ceux qui sont éloignés, exclus de la bibliothèque. D’où cette indifférence si fréquente pour l'accueil réel des usagers réels. Il s'agit de convertir (les non-usagers) plus que de servir (les usagers).

Étrange interprétation, fantasmatique, de l'ambition de démocratisation. Étrange position de la part des bibliothécaires, du moins dans leurs discours, qui jettent eux-mêmes un doute permanent sur l'achèvement de leurs missions. Alors que, aux États-Unis, la bibliothèque est motif de 
fierté - et ceci, loin de se fonder uniquement sur le nombre des usagers, mais en raison des objectifs et du rôle des établissements.

Le lent développement des bibliothèques municipales en France, les modalités de leur tardive expansion, l'ambiguité des discours des bibliothécaires pourraient ainsi avoir créé de nouveaux obstacles. Car, à comparer les argumentaires développés de part et d'autre de l'Atlantique, on trouve des oppositions radicales. La bibliothèque comme école après l'école, la bibliothèque pour toute la population sont des arguments que les bibliothécaires français ne veulent pas prendre à leur compte. Les bibliothèques sont ainsi coupées de leur enracinement politique (la formation des citoyens, le soutien à la vie démocratique) et fragilisées par une extra-territorialité (un service public national exercé au niveau local) qui porte en germe les limites de leur succès. Elles restent ainsi à l'écart, sans appui politique ni soutien populaire. Sans ennemis mais sans amis.

Le rapport des bibliothèques au territoire, à la population, aux lieux de pouvoir, aux pouvoirs eux-mêmes, est, on le voit, très différent dans les deux pays. Dans un cas (le cas américain), la Public Library a su s'implanter et se nourrir de cette implantation. Dans l'autre (le cas français), la bibliothèque semble restée " hors-sol ", sans racines ni terreau. Le chapitre suivant va examiner plus avant ce rapport des bibliothèques $\mathrm{au}(\mathrm{x})$ pouvoir(s). 
CHAPITRE III POUVOIRS ET BIBLIOTHĖQUES 


\section{CHAPITRE III POUVOIRS ET BIBLIOTHÈQUES}

A près cette analyse de l'origine, des racines, des bibliothèques publiques des deux pays, il convient d'étudier la façon dont ces modèles sont mis en œuvre. Dans ce chapitre, je souhaite explorer l'hypothèse que le point central de divergence pourrait bien être la place de ces bibliothèques dans le champ du pouvoir. Leurs relations aux pouvoirs. La prise en charge par les pouvoirs. Les contre-pouvoirs. Le pouvoir d’influence. Le pouvoir de décision.

En la matière, il est indéniable que la situation française et la situation américaine, que l'histoire française et l'histoire américaine ont de profondes différences. Pourtant, les racines sont communes, de deux pays démocratiques dont le pouvoir par le peuple appelle, exige des citoyens éclairés et, donc, de deux pays pour qui le partage du savoir, l'éducation, l'école sont des enjeux politiques primordiaux. Mais ce terreau commun est très vite partagé, comme par une ligne de séparation des eaux, en des développements divergents : en ce qui concerne notre problématique, quatre différences importantes peuvent être identifiées.

La première d'entre elles est bien sûr le fait que les États-Unis sont une fédération, où l'État fédéral est, depuis l'indépendance, considéré comme une institution potentiellement dangereuse dont il convient de se méfier et de se protéger ${ }^{204}$. De Jefferson à Nixon (pardon pour le rapprochement), le Big Government, voilà l'ennemi - à la brève exception du New Deal de Roosevelt où des conditions économiques et sociales dramatiques justifièrent, appelèrent l'intervention de l'État fédéral (les interventions de l'État, aujourd'hui dans la crise économique, sont vues comme des héritières de cette période si particulière). Il est à noter que cette tendance idéologique lourde n'interdit pas l'intervention de l'État fédéral ; mais elle la rend plus difficile car contraire au substrat constitutionnel.

204. « Le gouvernement le meilleur est celui qui gouverne le moins », dit une célèbre phrase de H. D. Thoreau. Dans son discours d'investiture, en 1801, Thomas Jefferson défend " un gouvernement sage et frugal, qui retiendra les hommes de se porter tort les uns aux autres et qui, pour le reste, les laissera libres de régler leurs propres efforts d'industrie et de progrès. » 
Point besoin de souligner la différence avec la France où, jusqu'à ces vingt dernières années, nul salut ne pouvait venir que de l'État.

$\mathrm{Si}$, dans les deux pays, les bibliothèques publiques sont des institutions locales, dépendant des pouvoirs locaux, elles sont nées telles aux États-Unis mais ont été, en France, voulues, inspirées, soufflées par l'action de l'appareil d'État, avant, là aussi, que les collectivités territoriales ne jouent pleinement leur rôle d'acteur politique. Les communes américaines voulaient des bibliothèques et ont été autorisées à en créer ; les communes françaises ne s'en souciaient guère et ont été incitées à en créer.

La troisième différence tient au caractère pas tout à fait public, vu par un œil français, de l'administration de ces Public Libraries. Même si leur origine locale implique une grande diversité de statuts, la plupart sont gouvernées par un Library Board, sorte de conseil d'administration, soit nommé par le maire soit élu par la population. C'est lui qui détermine la politique de la bibliothèque, politique mise en œuvre par le directeur de la bibliothèque, avec, on s'en doute, un jeu de pouvoir subtil et changeant entre le pouvoir politique (le Library Board) et le pouvoir scientifique (le bibliothécaire). En France, les bibliothèques municipales sont des services municipaux qui dépendent des élus et seulement d'eux.

Enfin, quatrième différence, les lois qui gouvernent les Public Libraries donnent soit aux élus locaux soit directement aux citoyens (par referendum) le pouvoir de décider des ressources des bibliothèques, en particulier lorsqu'il s'agit d'opérations spécifiques comme la construction d'un bâtiment. Les bibliothèques ont donc besoin du soutien de la population (Public Support) pour se développer. Et ont donc besoin de déployer toute une stratégie de communication, conviction, alliances, vis-à-vis de la population, des groupes qui la composent et des leaders qui l'influencent. Il s'agit de l'application normale de la Constitution américaine, qui entend que les politiques mises en œuvre le soient avec l'approbation des citoyens (The Consent of Governed). En France, seuls les élus ont le pouvoir de décision en matière budgétaire et le budget des bibliothèques n'est pas traité de façon singulière. Les élections municipales, tous les six ans, donnent quitus ou non de la politique suivie par les élus et des choix qu'ils ont faits, notamment en matière de bibliothèque - entre deux élections, l'usager-électeur n'a pas la parole.

Ce chapitre a donc pour propos d'analyser ces deux contextes et l'influence qu'ils peuvent avoir (ou avoir eue) sur le développement des bibliothèques. Avant d'entamer cette analyse, il convient sans doute de souligner 
la diversité des situations non seulement entre les deux pays, mais à l'intérieur de chacun d'eux. Quoi de commun entre la New York Public Library et la Rutherford Library (New Jersey, 18000 habitants), entre la Chicago Public Library et la Free Urbana Library (Illinois, 43000 habitants) ? Quoi de commun entre la Public Library d'une métropole, celle d'un comté, celle d'une petite ville, celle d'un état ? Quoi de commun entre la Bibliothèque municipale de Lyon et celle de Laon, entre la Bibliothèque de Limoges et celle de Larmor-Plage ? Beaucoup et peu : beaucoup d'objectifs partagés, d'outils communs, de procédures et de réglementation communes. Mais aussi, beaucoup de différences, des contextes politiques, économiques, sociaux différents, des populations dont les profils, les attentes et les souhaits ne sont pas les mêmes, des moyens sans comparaison possible, une image, des projets différents. C'est en gardant à l'esprit cette diversité qu'il convient d'entreprendre cette analyse.

\section{LES BIBLIOTHÈQUES ET LE POUVOIR CENTRAL}

Deux flux différents, on l'a vu : en France, jusque dans les années 1970, le flux est descendant, de haut en bas, de l'État vers les communes pour irriguer les régions françaises. Aux États-Unis, la jonction ne se fait pas entre niveaux de pouvoir mais par le biais du lobbying de l'ALA qui plaide auprès du gouvernement fédéral pour une aide en faveur des régions (surtout rurales) mal desservies, dans l'indifférence, semble-t-il, des états et des communes vis-à-vis de ce projet. Contrairement à la situation locale où les positions sont établies dans une rigidité structurelle, ce qui se passe au niveau central est marqué au cours de ce demi-siècle par une conjoncture changeante - sur les plans politique et idéologique.

\section{Émergence, résurgence et évaporation}

On peut dater des années 1930, des deux côtés de l'Atlantique, l'émergence d'une demande d'intervention du niveau central en faveur des bibliothèques. Émergence : les prémices souterraines sont plus anciennes. Mais, ici comme là-bas, les voix s'élèvent, plus nombreuses et plus fortes, à l'occasion de la crise, de la dépression et de la mise en place de politiques étatiques plus volontaristes. Le New Deal et le Front Populaire, pour faire vite, amènent au grand jour la question de l'égalité entre les territoires et entre les populations. La politique d'aide aux bibliothèques est fille de 
l'État-providence, qu'il se prononce ou non Welfare State. Des deux côtés de l'Atlantique, des demandes sont formulées dans les années 1930 pour que le niveau central ou fédéral prenne en charge ce dossier. En France, la multiplication des politiques publiques, après la Libération, est la traduction de cette confiance dans le rôle de l'État, seul à même d'assurer, selon la formule de François Bloch-Lainé, « un développement volontaire et organisé » du pays.

En France, c'est une succession de rapports qui suggèrent d'organiser les bibliothèques sous la houlette de l'État : une administration centrale, une loi sur les bibliothèques, l'organisation raisonnée du territoire sont les constantes que l'on retrouve dans les travaux de la Commission de la lecture publique (1929), dans le plan de Grolier (1938), dans le rapport d'Henri Vendel présenté au nom de l'ABF (1942) ou dans la note de Pierre Lelièvre sur l'« organisation d'un service de la lecture publique » (1944). Si les mêmes solutions sont avancées et répétées avec constance, c'est que l'analyse est, elle aussi, constante : les villes ne faisant rien pour leurs bibliothèques, c'est à l'État de prendre en charge leur développement. Le rapport La lecture publique en France, en $1948{ }^{205}$, est sans équivoque : "Les crédits qui peuvent leur être accordés [aux bibliothèques municipales] varient selon l'importance du budget municipal total et aussi malheureusement selon l'intérêt que portent les municipalités à leur bibliothèque. » A contrario, ce rapport attribue à l'État la paternité des progrès enregistrés : «Sans cette aide de l'État, il est à présumer que bien des travaux n'auraient jamais été entrepris et que bien des services nouveaux n’auraient jamais été créés. » En 1954, c'est le même constat désolé qui est dressé : « Dans les villes, la création des bibliothèques publiques, sections ou annexes pour enfants, dépend des municipalités. La Direction des bibliothèques de France ne peut intervenir que pour susciter de telles créations et apporter éventuellement une aide financière et technique. Un grand effort reste à faire et sur ce point la France marque un retard regrettable par rapport à l'étranger. ${ }^{206}$ »

Pour lancer l'impulsion nécessaire, moderniser, rationaliser, la jeune Direction des bibliothèques et de la lecture publique propose et fait adopter, en 1945, un plan d'aménagement du territoire, " un programme qui devait en fin de compte doter la France d'un réseau de bibliothèques suf-

205. La Documentation française. Rapport émanant de la jeune Direction des bibliothèques et de la lecture publique, qui avait pour principal objet de dresser un constat sévère (mais juste) de la réalité des bibliothèques municipales, pour conforter la Direction et son budget.

206. Cahiers des bibliothèques de France, 1954. 
fisamment dense pour que toute demande de livres quelle qu'elle soit et d'où qu'elle vienne fût satisfaite. ${ }^{207}$ » Ce programme comprend, à l'échelon départemental, les bibliothèques centrales de prêt, dont les huit premières furent créées par ordonnance du 2 novembre 1945, comme services extérieurs de l'État (ce qu'elles resteront jusqu'en 1986). Et « à l'échelon régional, une bibliothèque “centrale régionale” créée auprès d'une bibliothèque municipale classée ou d'une bibliothèque universitaire, chargée de prêter des ouvrages de référence (les usuels des grandes bibliothèques), de réorganiser le prêt interbibliothèques, de faciliter les recherches des travailleurs grâce à l'existence de catalogues collectifs analogues à ceux qui existent en Angleterre, en Allemagne, aux États-Unis [...]. » Créées sur le papier le 24 octobre 1945, ces neuf premières bibliothèques régionales ne virent jamais le jour, d'une part parce que l'échelon régional éveillait de malheureux souvenirs vichyssois ${ }^{208}$, d'autre part parce que leur rattachement à une bibliothèque existante posait de très sérieux problèmes fonctionnels et administratifs.

Cette première tentative de mise en place d'un schéma national n'a donc connu comme postérité que les bibliothèques centrales de prêt, chargées de la desserte des zones rurales. Les services structurants, destinés à animer le réseau des bibliothèques urbaines, n'ont pas vu le jour, et ont donc réduit le champ du dialogue à ces deux acteurs que sont l'État et les villes - deux acteurs aux objectifs, aux moyens, au calendrier si différents. Pour les bibliothèques municipales, aucune structure d'appui ou de coordination n'est prévue.

Aux États-Unis, évidemment, les attendus sont tout différents. Il ne s'agit pas de demander au gouvernement fédéral de se substituer à des villes défaillantes mais de contribuer à l'égalité d'accès sur tout le territoire, notamment en combattant l'injuste situation des zones rurales, dont les ressources insuffisantes ne permettent pas de créer des Public Libraries, comme les villes le font ${ }^{209}$. En 1934, l'ALA adopte un «Plan national pour les bibliothèques » qui demande une aide fédérale pour les zones rurales et la création d'une agence fédérale, chargée d'attribuer et contrôler cette

207. La lecture publique en France. La Documentation française, 1948.

208. Pascal Ory souligne la volonté de «l'Assemblée constituante, visant à éradiquer jusqu'aux moindres traces de la régionalisation, soupçonnée d'esprit “vichyste”. " ("Le rôle de l'État : les politiques du livre ». Histoire de l'édition française, tome 4, sous la dir. d'Henri-Jean Martin et Roger Chartier, Fayard-Cercle de la Librairie, 1992).

209. Pour donner une idée de l'écart entre la situation américaine et la situation française, on comptait environ 7400 Public Libraries en 1945 et environ 400 bibliothèques municipales en 1948. 
aide. Cette initiative, absolument révolutionnaire dans ce pays fédéral, provoqua un vif émoi chez les membres de l'ALA qui protestèrent en grand nombre ${ }^{210}$, à la fois sur le principe d'une aide fédérale, qui impliquait un contrôle fédéral sur l'emploi de cette aide, mais aussi parce que l'association entrait ainsi dans le jeu politique ${ }^{211}$. Cette demande est alors abandonnée par l'ALA - au reste, la conjoncture économique rendait son adoption par le gouvernement fédéral bien improbable.

De façon moins directe, les bibliothèques et les bibliothécaires ont profité d'une autre aide, celle des programmes fédéraux destinés à soutenir puis relancer l'activité du pays pendant la Dépression. Intégrée dans des programmes non spécifiques (ceux de la Federal Emergency Relief Administration puis de la Works Progress Administration), cette aide fédérale rencontra une moindre opposition - elle fut largement définie en liaison avec l'ALA et son puissant directeur exécutif, Carl Milam. Au demeurant, ses objectifs rejoignaient ceux des bibliothécaires : améliorer les services des bibliothèques, surtout en zones rurales, encourager l'éducation des adultes et, objectif évidemment consensuel, redonner du travail aux bibliothécaires chômeurs ${ }^{212}$. Cette collaboration entre l'ALA et le gouvernement fédéral fut un premier pas pour reconnaître les Public Libraries non seulement comme des institutions (administratives) locales mais comme une institution (symbolique) nationale.

Après la guerre, il n'est donc pas étonnant que l'ALA ait renouvelé sa demande d'une aide fédérale, qu'elle appuie désormais d'un intense lobbying auprès du Congrès. À partir de 1947, tous les ans, l'ALA intervient auprès des parlementaires pour faire adopter cette aide fédérale - en vain. La dixième année, en 1956, est enfin adopté le Library Services Act (LSA), maigrement doté par le gouvernement (7 millions de dollars, dont le Congrès n'inscrivit que 2 millions au budget). L'objectif est de fournir aux 25 millions d'Américains dépourvus de Public Libraries et aux 50 millions qui n’ont à leur disposition que des services « inadéquats » un service de bibliothèque digne de ce nom. L’aide fédérale n’est donc pas, loin

210. Oliver Garceau estime à $25 \%$ les adhérents de l'ALA qui protestèrent par écrit, proportion considérable. (Oliver Garceau. The Public Library in the Political Process, op. cit., p. 177)

211. Dennis Thomison. A History of the American Library Association (1876-1972). Chicago, ALA, 1978. Michael Blayney ajoute un autre argument, celui que l'aide fédérale tuerait l'investissement local, en semblant dédouaner l'échelon local des responsabilités assumées jusque-là. (Michael Blayney. "Libraries for the Millions: Adult Public Library Services and the New Deal". Journal of Library History, Summer 1977)

212. On estime à 2500 le nombre de bibliothécaires sans emploi à l'été 1933, la moitié ayant été licenciés, l'autre étant des jeunes professionnels récemment diplômés et ne trouvant pas d'embauche. 
de là, inconnue des bibliothèques américaines. Nous reviendrons plus loin sur les argumentaires employés, tant par les promoteurs que par les opposants à cette aide - et même par ses bénéficiaires potentiels, l'Indiana et le Wyoming refusant d'en bénéficier ${ }^{213}$.

Depuis les années 1950, donc, une aide fédérale est instituée. Elle connaîtra des développements successifs dans son contenu, élargissant son objet aux zones urbaines et à la construction de bâtiments en 1964 (Library Construction and Services Act, LCSA ${ }^{214}$ ), puis à l'informatisation et à la mise en réseau des établissements en 1996 (Library Services and Technology Act), cette dernière aide étant étendue à tous les types de bibliothèque et non plus seulement aux Public Libraries.

Même si les sommes attribuées sont relativement modestes, 626 millions de dollars entre 1956 et 1974, les fonds attribués restent toujours menacés : à partir de 1973 le gouvernement tente de mettre fin au LCSA, sauvé d'année en année par le Congrès. Car la volonté politique varie en fonction de la conjoncture, les Congrès à majorité démocrate se montrant régulièrement plus généreux que les Congrès à majorité républicaine, toujours désireux de diminuer le pouvoir et l'influence de l'État fédéral. Après la lutte de Nixon pour réduire le Big Government, les années ReaganBush sont, elles aussi, clairement défavorables à l'investissement de l'État fédéral. Sous Clinton, une majorité républicaine est élue au Congrès dès 1992 sur la base du programme Contract with America qui met en avant la responsabilité des individus, des familles et des niveaux de gouvernement les plus bas (états, comtés et villes), considérant l'intervention fédérale comme illégitime. Si l'aide fédérale est donc effective depuis un demi-siècle maintenant, elle est en même temps toujours menacée, peu élevée et soupçonnée d'illégitimité.

Côté français, c'est évidemment un autre discours que l'on entend. L'État jacobin est à la fois le garant de l'intérêt général et le détenteur de l'expertise. Il lui revient donc d'orienter l'action des villes : « Orienter l'action de la société civile locale en faisant prévaloir le bon sens et la raison centrale. Tout se passe en effet comme si, abandonnée à ses propres intuitions, la société locale n'était capable que de déraison, ou, du moins, était inapte à trouver par elle-même la solution à ses propres pro-

213. Loleta D. Fyan. "Progress and Policies under the Library Services Act”. The Library Quarterly, oct. 1957. Ces deux états, dit-elle, montrèrent une " insurmontable opposition » à ce programme fédéral de subventions.

214. De fait, par le vote d'amendements successifs, le LCSA devint une sorte de dispositif fourre-tout destiné à aider le développement des Public Libraries. 
blèmes. D’où l'extraordinaire volonté de "pédagogie", sincère ou calculée, que l'on rencontre dans l'administration française à l'égard des acteurs locaux », écrivent en 1970 Pierre Grémion et Jean-Pierre Worms ${ }^{215}$.

Pour tenter de faire évoluer la situation des bibliothèques municipales, tributaires de l'action ou de l'inaction des élus locaux, un dispositif d'incitation est élaboré au niveau gouvernemental avec le soutien actif du groupe professionnel des bibliothécaires. Dispositif politique (appeler les villes à leurs responsabilités), pédagogique (diffuser le modèle de bibliothèque publique) et financier (subventionner les dépenses de fonctionnement et d'équipement). Mis en place avec de vrais moyens à partir de 1968, à la suite du comité interministériel sur la lecture publique ${ }^{216}$, ce dispositif portera ses fruits à partir de la fin des années 1970 quand il trouva - enfin ! - des interlocuteurs locaux, les élus issus des élections municipales de 1977, souvent de gauche, souvent jeunes, souvent intéressés par le domaine éducatif et culturel. Les subventions de l'État sont un puissant levier à la création et au développement des bibliothèques municipales : levier financier, bien sûr, mais aussi symbolique car recevoir l'aval ministériel est la légitimation d'un projet local et de ceux qui le portent ${ }^{217}$. Ce système fonctionnera pendant une vingtaine d'années.

Lui succède, à partir de 1986 dans le cadre de la décentralisation, un système légèrement modifié ${ }^{218}$. Légèrement mais pas radicalement modifié : alors que la décentralisation appelait leur disparition, le travail pédagogique et l'aide financière perdurent sous la forme dérogatoire du "Concours particulier des bibliothèques municipales ». C'est l'échelon central lui-même (la Direction du livre et de la lecture) qui considère que la tâche de mise à niveau des établissements est loin d'être achevée et que, en conséquence, l'État ne peut se priver du levier que sont les subventions spécifiques. Son plaidoyer est entendu : les subventions destinées au fonctionnement et à la construction des bibliothèques n’ont pas

215. « L'État et les collectivités locales ». Esprit, janvier 1970.

216. Dont le rapport est publié sous le titre La lecture publique en France, La Documentation française, 1968.

217. Anne-Marie Bertrand. Les villes et leurs bibliothèques, op. cit.

218. C'est bien 1986 qui marque le changement des procédures, alors que le nouveau ministère Lang et la Direction du livre alors dirigée par Jean Gattégno assument dès 1982 la politique suivie avant eux : « Non seulement la DLL a pu financer en 1982 près de $60000 \mathrm{~m}^{2}$ de bibliothèques municipales, contre $13000 \mathrm{~m}^{2}$ en 1981, mais la politique qu'elle a menée a eu pour effet capital de redonner aux élus le désir et la volonté d'équiper leur commune, donc de faire surgir des programmes et des plans là-même où jusque-là l'intérêt d'un service public de la lecture n'était pas perçu » (Brochure du ministère de la Culture, 2 ans de politique culturelle 81-83. Livre). Où l'on trouve plus que des traces du rôle d'entraînement de l'État, capable de donner ou redonner « aux élus le désir et la volonté » de construire une bibliothèque. Phrase qui n'aurait pas de sens aux États-Unis. 
été mêlées (globalisées) à l'ensemble des aides de l'État décentralisées auprès des collectivités territoriales sous la forme de la dotation générale de décentralisation. Elles continuent à être spécifiquement attribuées sur projets pour les aides à l'équipement des bibliothèques. Cette intervention étatique perpétuée est complétée du lancement de programmes dotés de subventions spécifiques (les bibliothèques municipales à vocation régionale, grands projets en région en 1992, les " ruches ", bibliothèques de proximité en 2003). En France, même si la conjoncture politique évolue nettement au cours du dernier demi-siècle, on constate donc une permanence dans l'aide de l'État et dans la légitimité qui lui est reconnue.

\section{Standards, normes et programmes}

Même si les argumentaires justifiant l'intervention de l'échelon central ne sont pas les mêmes en France et aux États-Unis, ils se rejoignent sur un point : il s'agit de développer les bibliothèques, en les mettant à niveau (France) ou en favorisant l'égalité d'accès à l'éducation (equalization of educational opportunity, États-Unis) ${ }^{219}$. Dans les deux cas, il convient donc de déterminer un niveau d'intervention : soit un plancher en dessous duquel les services sont considérés comme de qualité insuffisante et donc éligibles à l'aide fédérale ; soit un optimum vers lequel tirer les projets municipaux et les rendre éligibles aux subventions de l'État. Cette question a été traitée tout à fait différemment des deux côtés de l'Atlantique. En France, c'est l'État qui élabore et diffuse des documents techniques et pédagogiques. Celui de ces documents qui a eu la plus riche postérité est sans doute " Bibliothèques municipales, construction, équipement », diffusé pour la première fois en 1975 et « destiné à faciliter la tâche des services communaux ou préfectoraux, des bibliothécaires et des architectes, chargés d'établir ou d'approuver les plans de bâtiments à construire [...]. Plutôt que de proposer des programmes-types, il s'agit d'offrir le plus d'éléments d'information et de réflexion possible de façon à permettre d'établir un programme particulier dans une situation donnée. Le document devra néanmoins présenter à titre indicatif des normes en surfaces, en livres et en personnel, ainsi que des recommandations d'ordre technique. ${ }^{220} »$

219. C'est en tant qu'outil éducatif que les bibliothèques sont dignes d'être soutenues et les deux expressions equalization of educational opportunity et equalization of library opportunities sont employées.

220. «Groupe de travail sur les bibliothèques municipales. Document préparatoire ». Service de la lecture publique, novembre 1973. 
Les « normes » sont ainsi destinées à chiffrer les objectifs à atteindre, surtout pour les bâtiments, mais aussi pour les collections (et donc les crédits d'acquisition) et le personnel (effectif et qualification). Cette analyse chiffrée permet d'estimer le déficit en locaux, en collections et en emplois des bibliothèques municipales par rapport à l'optimum considéré. Elle peut donc être utilisée (et elle le sera) vis-à-vis de deux types d'interlocuteurs : au sein du ministère, elle permet de chiffrer le " retard français » et de plaider pour avoir un bon budget d'équipement ${ }^{221}$; aux villes, elle précise le seuil normatif qu'il faut chercher à atteindre et en regard duquel le projet de construction va être débattu. Le terme de « normes » est évidemment ambigu. Qui parle de normes, parle logiquement d'obligations à respecter, de seuils à atteindre : ici, le ministère publie des "normes » à " titre indicatif ", c'est-à-dire sans caractère contraignant. Ce document affiche ainsi non des obligations mais des objectifs, vers lesquels il faut tirer les villes : il y a un écart entre les projets initiaux des villes (qui s'inspirent de ce qu'elles connaissent) et les projets acceptés par le ministère (qui se rapprochent de ce qui est souhaitable), les « normes » sont destinées à combler cet écart. Elles sont donc forcément ambitieuses, puisqu'elles sont un moteur, un levier. « Elles ne doivent pas être conçues à partir d'une pénurie », dit le Service de la lecture publique en décembre 1973, lors de la première réunion du groupe qui les élabora. Mais elles ne sont pas impératives.

Aux États-Unis, où l'équivalent de la Direction des bibliothèques puis de la Direction du livre n'existe pas ${ }^{222}$, c'est l'ALA et, en première ligne, sa division des Public Libraries (en 1950 Public Libraries Division, puis à partir de 1959 Public Library Association, PLA) qui élaborent des « standards ", indissociables de l'organisation et de la planification : on se souvient que la Public Library Inquiry, en 1950, plaidait fermement pour le rassemblement des bibliothèques en unités plus larges, seules à même de dispenser un service de niveau satisfaisant. Il est, bien sûr, ironique de constater que ce qu'un pays centralisé avec une longue tradition de programmes pluri-annuels n'a pas réussi à faire pour ses bibliothèques, un pays fédéral et aux collectivités autonomes l'a pour une large part réalisé.

Pourtant, l'ironie n'est pas de mise : car un tel regroupement de bibliothèques a bel et bien été proposé en France. Il s'agissait des bibliothèques

221. Le rapport Vandevoorde, Les bibliothèques en France, Dalloz, 1982, avait dans l'esprit de ses auteurs la mission de montrer chiffres en mains la situation très préoccupante des bibliothèques. 222. Les services administratifs qui existent ont un rôle de gestion des budgets fédéraux et non un rôle d'incitation politique comme en France. 
de secteur, promues dans les années 1960 et 1970 avec un succès d'estime réel tant elles étaient une réponse à une vraie question, l'émiettement des moyens et l'absence d'intérêt de beaucoup d'élus locaux engendrant une grande inégalité de l'offre à travers tout le territoire. Les attendus étaient convaincants : "Le projet de bibliothèque de secteur repose sur l'idée générale suivante : il ne doit pas y avoir une lecture publique urbaine et une lecture publique rurale. Tous les citoyens quels que soient leur âge, leur situation sociale ou familiale, leur lieu de travail, de repos ou de loisir, leur niveau de culture générale, technique ou professionnelle doivent avoir accès librement au livre et aux autres moyens de formation et de distraction dans les mêmes conditions, avec les mêmes facilités. ${ }^{223}$ » Mais ce projet ne vit pas le jour car il était inadapté à la réalité géopolitique française : ici, pas d'états pour jouer les relais de l'État et prendre en charge, sur leur territoire, l'organisation du système des bibliothèques. $\mathrm{Ce}$ ne sont pas seulement les échelons politiques qui diffèrent mais, évidemment, leur capacité à inter-agir.

Nouveau contre-pied : les travaux nombreux, l'abondante production des bibliothécaires américains en matière de standards s'achèvent dans une auto-dissolution du concept même de standards. À partir du début des années 1970, la PLA considère qu'il n'est plus approprié d'élaborer des standards qui seraient valides pour toutes les Public Libraries. La bonne méthode, suggère-t-elle, est de donner des outils aux bibliothèques pour que chacune puisse élaborer des standards appropriés aux besoins de la population qu'elle a à desservir. Évaluant cette idée à la demande de la PLA, Mary Jo Lynch et Ralph Blasingname écrivaient en 1974 : « Ce dont les bibliothécaires ont besoin maintenant, ce sont des outils qui leur permettent d'analyser une situation, de définir des objectifs, de prendre des décisions et d'évaluer leurs activités [...]. Penser à organiser l'avenir plutôt que revenir sur le passé. Penser à la gestion de la bibliothèque plutôt qu'à la comparaison avec d'autres bibliothèques. S'intéresser aux résultats, c'est-à-dire ce que l'usager obtient de la bibliothèque plutôt qu'aux moyens, c'est-à-dire le personnel, les collections et le bâtiment. ${ }^{224}$ "

Cette nouvelle logique sera mise en pratique dans le Planning Process for Public Libraries, publié en 1980 par l'ALA, qui construit son propos sur la nécessité pour les bibliothèques de connaître les besoins de la com- 
munauté qu'elles ont à desservir et d'y répondre : il s'agit bien de définir " des standards appropriés à la situation locale » et à elle seule ${ }^{225}$. Est-ce à dire que les bibliothécaires américains renoncent à partager valeurs, objectifs, politiques et méthodes ? Le débat est ouvert ${ }^{226}$ et le passage de standards nationaux à des outils locaux n'a pas convaincu l'ensemble de la profession.

Malgré, on le voit, quelques grincements de dents, la logique s'est cependant imposée, avec ses deux tendances lourdes : d'une part, le lien étroit entre les objectifs que se fixent la bibliothèque et la situation locale ; d'autre part, l'accent mis sur l'évaluation des résultats plutôt que sur le niveau des moyens. Réponse tardive à la déception que provoqua chez les bibliothécaires la publication de la Public Library Inquiry ? Pour ne plus être confrontés à cet écart entre les discours et la réalité, il faut rester proches de la réalité et la connaître bien. La logique de cette démarche peut donc être vue comme un glissement de la définition des objectifs à celle des rôles et de la définition des rôles à l'évaluation des résultats. Le tout se faisant dans une relation étroite avec le contexte local. Sans renier la valeur fondamentale : Service to all.

\section{L'intervention de l'État central comme chevalier blanc ?}

L'intervention de l'État au niveau central, qu'il soit national ou fédéral, a connu des débuts comparables dans les deux pays : la montée en puissance de l'État-providence dans les années 1950 et 1960 s'est traduite par une nouvelle implication en faveur des bibliothèques publiques. Mais les différences sont apparues rapidement : dans un cas, aux États-Unis, l'aide fédérale a soutenu le développement d'un échelon intermédiaire, les State Libraries, agences des états, qui l'a largement relayée, organisée et amplifiée. Avec la conséquence que cette amplification des moyens a engendré une pratique de la planification et de l'évaluation qui s'est largement

225. Edward J. Elsner. “The Evolution of PLA's Planning Model”. Public Libraries, July-August 2002. Les versions successives des manuels de la PLA vont toutes dans le sens d'une singularisation des établissements, centrés sur leur propre population à desservir (a community centered planning process, résume Edward Elsner). Après le Planning Process de 1980, il s'agit du Planning and Role Setting for Public Libraries (1987), de Planning for Results: A Public Library Transformation Process (1998) et de The New Planning for Results: A Streamlined Approach (2001).

226. Mary N. Maack, "Public Libraries in Transition", op. cit., dit que beaucoup de directeurs de bibliothèque se trouvaient mal à l'aise (uncomfortable) dans cet abandon de standards nationaux. Des compromis furent passés sur ce point (notamment dans Planning and Role Setting Manual, en 1987), mais sans renoncer à mettre en avant la singularité de chaque situation : Excellence must be defined locally. 
recentrée sur l'échelon local. En France, l'aide étatique a peiné à trouver des relais, ses seuls bénéficiaires et partenaires, les villes, jouant un jeu purement local - sans le détour américain par l'organisation de réseaux régionaux. L'intervention de l'État est donc toujours attendue dans un dialogue exclusif, puisqu'elle n'a pas d'alternative.

Un second constat peut être tiré de l'analyse de ces aides, discutées ici, demandées là. Car les États-Unis, pays fédéral, répugnent à l'intervention du niveau national. Les péripéties du lancement laborieux du LSA sont éclairantes : entre 1947, où l'ALA intervient pour la première fois auprès des parlementaires en faveur d'une aide fédérale aux bibliothèques, et 1956 où elle obtient gain de cause, les débats ont été rudes. La question centrale est celle de la légitimité des acteurs : en quoi l'ALA est-elle légitime pour défendre cette demande ? En quoi l'aide fédérale serait-elle une réponse légitime ? Le demandeur, l'ALA, a quelquefois suscité l'ironie des parlementaires : qu'est-ce qui l'autorise à demander l'aide fédérale, alors même que ni les états, ni les comtés, ni les villes ne la demandent ${ }^{27}$ ? Qu'est-ce donc que ces " pickpockets professionnels ", ce "petit groupe professionnel, avec une minuscule poignée de voix, qui veut pousser le grand Congrès des États-Unis à sauter dans le cerceau pour obéir à ses instructions ou désirs ${ }^{228}$ » ? Au-delà de l'agacement, qui fait sans doute partie du jeu de la négociation, la question de fond qui est posée est celle de la légitimité de l'intervention fédérale.

Douglas Raber, qui a analysé finement ces débats, souligne que tous les parlementaires considèrent les Public Libraries comme dignes d'intérêt et de soutien. Mais qui doit les soutenir ? Des ressources existent au niveau local, argumentent les opposants au projet : c'est à ce niveau qu'elles doivent être mobilisées. L'intervention de l'État fédéral n'aboutirait qu'à augmenter les impôts et à déresponsabiliser les citoyens. Position traditionnelle des républicains, qui est combattue par les démocrates (mais pas par tous), décidés, eux, à promouvoir le principe d'égalité d'accès (equal opportunity). L'intérêt général n'est pas la somme des intérêts particuliers et la communauté a « l'obligation morale de compenser les différences injustes entre les individus. » Cette frontière argumentaire, traditionnelle entre les deux grands partis américains, se prolonge jusque

227. "Not a single state has transmitted to the committee in writing or even orally a request for this program”, argumente le Républicain Walter Judd en 1950 (cité par Douglas Raber, "Ideological Opposition to Federal Library Legislation: The Case of the Library Services Act of 1956", Public Libraries, May/June 1995)

228. Ibid. 
dans les années 1990 : pour l'administration Reagan et Bush, le LCSA n'a pas de raison d'être, car les Public Libraries ne sont pas du ressort de l'État fédéral. L'aide a pourtant été maintenue, car elle est peu coûteuse et efficace ${ }^{229}$ - le retour sur investissement est bon.

En France, ce débat est hors de propos : la légitimité de l'intervention de l'État est assise depuis longtemps. Ce qui fait dissension, pour les bénéficiaires potentiels, c'est l'importance de l'aide de l'État - toujours considérée comme insuffisante. Du côté gouvernemental ou parlementaire, les bibliothèques sont un sujet trop mince pour susciter des débats comparables à ceux que l'on vient d'évoquer. Rappelons simplement que le volet financier du plan de développement de la lecture publique, en 1968, ne fut ni publié ni appliqué - Olivier Guichard, alors ministre de l'Éducation et donc en charge des bibliothèques publiques, se contenta de regretter, lors du débat parlementaire sur le budget 1970, que les bibliothèques soient les «victimes privilégiées des conjonctures budgétaires malheureuses. » (Assemblée nationale, séance du 12 novembre 1969). Le septennat de Valéry Giscard d'Estaing est, lui, plus politique de ce point de vue : si les budgets disponibles pour subventionner les constructions des bibliothèques municipales vont diminuant d'année en année ${ }^{230}$, le ministère n'en accuse pas la conjoncture mais assume le refus de l'État de s'impliquer. Les bâtiments ne sont pas un élément essentiel du développement des bibliothèques, dit ainsi le rapport Granet, qui décide de « privilégier l'aide au livre par rapport à l'aide à la pierre ${ }^{231}$ »; une politique incitative de l'État est dépassée, estime pour sa part Jean-Philippe Lecat, ministre des Affaires culturelles : «En ce qui concerne l'action culturelle, l'époque où l'État avait un rôle d'entraînement à l'égard des collectivités est dépassée ». Cette brève parenthèse giscardienne (libérale) mise à part, la légitimité des aides de l'État aux bibliothèques publiques n'a jamais été mise en cause.

Il est clair que l'on ne peut comprendre la politique américaine sans la relier à l'intense activité de lobbying menée par l'ALA. Nous y reviendrons. Ici, contentons-nous de constater que, en France, ce travail de promotion, d’influence, de conviction, s'est plutôt fait au sein même de l'appareil administrativo-politique qui a conçu, en lien avec les avant-gardes du

229. Peter Fuller. “LSA and LCSA, 1956-1973: A Legislative History”. Library Trends, July 1975.

230. Ils permettent de subventionner $46700 \mathrm{~m}^{2}$ de constructions en 1974, et seulement $13000 \mathrm{~m}^{2}$ en 1981.

231. Pour une politique du livre : rapport à monsieur le Premier ministre. La Documentation française, 1975. 
groupe professionnel, un dispositif pédagogique, technique et financier en faveur du développement des bibliothèques publiques. Porte-parole des besoins de la population en matière de bibliothèques, ce segment administratif s'est régulièrement appuyé sur l'ABF. La décentralisation, en déconcentrant le pouvoir de décision au niveau régional (les Directions régionales des affaires culturelles), a privé l'échelon central de ce rôle de porte-parole. Les parlementaires, les élus, les citoyens : en regard de la situation américaine, tous les appuis possibles semblent frappés de mutisme.

Dernière question à poser : de quelle influence parle-t-on ? Uniquement en termes de moyens ou également en termes de projets ? Autrement dit, l'intervention de l'échelon central a-t-elle eu des effets surtout quantitatifs ou a-t-elle joué aussi sur les objectifs, l'organisation, l'activité des établissements ? Il semble que dans les deux cas, on peut dire que l'influence a été à la fois quantitative et qualitative. Quantitative comme soutien au développement, dans les deux pays. Qualitative de façon dissemblable. Aux États-Unis, le passage obligé de l'aide fédérale par les State Libraries a orienté tout le dispositif vers l'organisation de réseaux et de services communs, l'activité et les objectifs de chaque bibliothèque étant définis au niveau local. En France, où en 1945 tout était à faire ${ }^{232}$, l'échelon central, avec le relais actif de l'Inspection générale des bibliothèques, a propagé un véritable modèle de bibliothèque : par le biais des " normes » évoquées plus haut, c'est la création des sections pour les enfants, puis l'introduction de disques et de films, puis l'adoption de l'outil informatique (les systèmes de gestion de bibliothèque), puis des documents numériques qui ont été, tour à tour, encouragés et soutenus. En somme, c'est le modèle de la médiathèque qui a été promu sur l'ensemble du territoire. Mais ce modèle n'a pas été imposé : il a été suggéré, soutenu, encouragé - et n'a connu une telle diffusion que parce qu'il a rencontré l'intérêt des élus locaux et le succès public (l'intérêt des élus locaux parce que le succès public, pourrait-on écrire plus justement).

\section{LE POUVOIR À L'ÉCHELON LOCAL}

Ici aussi, il convient sans doute de rappeler la diversité des situations, non seulement entre les deux pays mais à l'intérieur même de chacun des pays étudiés. Si, en France, c'est le facteur de la taille qui provoque les 
différences (en pouvoir, expertise, projet) alors que la structure administrative est uniforme, aux États-Unis, la taille, le type et même le pouvoir des collectivités sont très variés : d'un état à un comté, d'une ville à pouvoir politique fort à celle où il ne joue qu'un rôle de gestion, l'éventail est large. En 1993, des 9097 Public Libraries recensées, 59 \% étaient sous juridiction municipale, $11 \%$ dépendaient des comtés, $12 \%$ d'associations (non-profit corporations), $4 \%$ de l'administration scolaire (school districts), $9 \%$ de juridictions spéciales (independent taxing districts, souvent en banlieue, elles correspondraient à nos anciens Sivom) et $4 \%$ avaient un régime mixte, dépendant de plusieurs juridictions ${ }^{233}$.

Mon propos n'étant pas de faire une description de cette situation compliquée mais de tenter de l'analyser en regard de la situation française (et réciproquement), je vais traiter cette question sous trois angles : la place du pouvoir politique (les élus) d'une part, l'exercice du pouvoir par le Library Board d'autre part, enfin les sources de financement.

\section{Le pouvoir politique}

Dans les deux pays, les bibliothèques publiques sont des institutions locales dépendant des élus locaux. « La Public Library aux États-Unis est aujourd'hui d'abord une institution locale », affirme sans ambiguïté Robert Leigh ${ }^{234}$. En France, on l'a vu, la traditionnelle demande d'État fait que l'affirmation est moins claire, plutôt empesée, interrogative : "Certains prôneraient volontiers la totale prise en charge par l'État de la lecture publique. Ne serait-ce pas faire fi de la collectivité locale, qui peut considérer comme lui revenant en propre une action étroitement liée à la politique éducative, sociale et culturelle de la commune ? ${ }^{235}$ »

Les relations entre pouvoirs locaux et bibliothèques s'exercent très différemment en France et aux États-Unis : dans le premier cas, les bibliothèques municipales sont tout simplement des services municipaux, dont le personnel, le budget, la politique sont définis et décidés par les élus locaux. Aux États-Unis, la majorité des Public Libraries est administrée par des Library Boards : on parle souvent de la «situation duale » des Public Libraries, services municipaux dont le personnel, la politique et

233. Alice Gertzog, Edwin Beckerman. Administration of the Public Library, op. cit. En 1993, les États-Unis comptaient 7175 villes et 3043 comtés. La structure du pouvoir dans les comtés étant comparable à celle des villes, je ne traiterai que de ces dernières.

234. Robert D. Leigh. The Public Library in the United States, op. cit.

235. Alice Garrigoux. La lecture publique en France. La Documentation française, 1972. 
même quelquefois le budget échappent au pouvoir local - la spécificité des Library Boards et leur influence sur le développement des bibliothèques seront évoquées plus loin.

Que signifie pour les villes ce partage du pouvoir ? Sauf dans les cas (très minoritaires) où le Library Board a légalement autorité pour lever l'impôt, les élus ne sont impliqués dans la vie de la bibliothèque que dans deux circonstances : le vote du budget et la désignation des membres du Library Board. L'expression « semi-indépendante » est souvent employée pour caractériser la place de la Public Library vis-à-vis du pouvoir municipal. Cette distance a été analysée comme une des faiblesses des Public Libraries car l'éloignement engendre le désintérêt. Héritage des social libraries, les Library Boards sont aussi une manifestation de défiance visà-vis du monde politique. Mais cette défiance pouvait se justifier au XIXe siècle et peut, au contraire, avoir aujourd'hui des effets désastreux : «Dans la plupart des cas, ce statut a été adopté à une époque où les Public Libraries étaient dans l'enfance et où les élus locaux étaient réputés pour leur incompétence et leur corruption. Tant les bibliothèques que les pouvoirs locaux ont fait des progrès considérables depuis ces débuts. À une époque, il a pu sembler raisonnable de protéger la bibliothèque et le bibliothécaire du politique, mais c'est déraisonnable aujourd'hui. La Public Library moderne fait clairement partie du système politique », analyse ainsi James Ubel, dès $1962{ }^{236}$. Cette distance au pouvoir politique, longtemps entretenue, a longtemps engendré des budgets faibles : à la fin des années 1940, Robert Leigh avance un chiffre de $2 \%$ du budget municipal et parle de "l'insignifiance fiscale » des bibliothèques - l'avantage, remarque-t-il aussi, c'est que ces budgets médiocres sont revotés sans encombre d'année en année ${ }^{237}$. Les Library Boards, jadis protecteurs, peuvent aujourd'hui devenir des obstacles à l'établissement de relations étroites avec le pouvoir local.

L'implication des bibliothèques - et des bibliothécaires - dans la vie politique locale fait aujourd'hui l'objet d'un discours unanime : tout en se gardant, bien sûr, d'adopter une posture partisane, le bibliothécaire doit établir des liens étroits, confiants et que l'on espère fructueux avec le monde politique local, les élus comme les administratifs. Il ne suffit pas de plaider, une fois par an, pour avoir un bon budget, il faut, toute l'année, se faire connaître et faire reconnaître la Public Library. Le problème, génère qu'une basse température lors des débats budgétaires (low cost, low temperature). 
analyse Edwin Beckerman, est de trouver la bonne distance : « Certains bibliothécaires ou administrateurs semblent penser que la neutralité de la bibliothèque est quelque peu compromise si les responsables de la bibliothèque tentent d'acquérir de l'influence sur les politiques. Ces craintes peuvent paraître déplacées, mais ne sont pas tout à fait sans fondement. En un sens, le lobbyiste de la bibliothèque a besoin d'être comme les bons journalistes qui entretiennent une relation amicale avec les politiciens tout en restant à distance. ${ }^{238}$ »

Depuis deux ou trois décennies, un des atouts pour être reconnu, crédible, efficace sur la scène politique locale est l'usage par les bibliothécaires de la planification. « À cause de cette attention plus grande portée à la planification, en particulier aux méthodes de la PLA, les bibliothécaires ont une idée plus précise de leurs buts, ont développé de meilleurs outils d'évaluation et sont capables de mieux expliciter leurs objectifs opérationnels que dans le passé », écrit encore Edwin Beckerman. En effet, un des handicaps des Public Libraries (mais c'est vrai aussi, bien sûr, pour les bibliothèques municipales) est la difficulté à mesurer, montrer, rendre visibles les services rendus par la bibliothèque. Des citoyens mieux informés, des adultes en formation, des étudiants soutenus dans leur cursus, des immigrés apprenant leur nouveau pays, des enfants familiarisés avec les livres, des foyers non équipés profitant d'Internet : tous ces effets de la bibliothèque, et bien d'autres, sont difficiles à faire valoir. Les hommes politiques ne sont pas hostiles aux Public Libraries, ils sont même plutôt bienveillants ${ }^{239}$. Mais pour passer de la sympathie à l'intérêt actif, les analystes et les bibliothécaires semblent penser que la meilleure façon d'avoir de l'influence est de s'en remettre au « bouclier » traditionnel qu'est le Library Board et au soutien de la population.

La situation est toute différente en France. Les bibliothèques municipales sont clairement et absolument des services municipaux ${ }^{240}$. C'est le

238. Edwin Beckerman. Politics and the American Public Library: Creating Political Support for Library Goals. The Scarecrow Press, 1996, p. 27. Il parle, bien sûr, des journalistes américains.

239. Edwin Beckerman écrit encore : «En général, les hommes politiques sont bien disposés à l'égard des bibliothèques, comme ils le sont à l'égard de Dieu, de la maternité, du base-ball et (aije besoin de le dire) de la tarte aux pommes. » Oliver Garceau le disait déjà, cinquante ans plus tôt : «Personne ne s'oppose à la bibliothèque ; presque tout le monde l'approuve. Mais, avec la même unanimité, presque personne ne veut payer beaucoup pour elle. Ce que les bibliothécaires combattent, c'est l'indifférence. » (Oliver Garceau. The Public Library in the Political Process, op. cit., p. 111)

240. L'intercommunalité va sans doute changer non seulement l'organisation mais le poids des bibliothèques dans le système politique local. Il est trop tôt, aujourd'hui, pour en faire une analyse historique. 
maire et le conseil municipal qui décident du budget alloué, des postes créés, qui recrutent le personnel, qui décident des horaires d'ouverture, du tarif des adhésions, de la création d'un nouveau service, de la construction d'un nouveau bâtiment, etc. Il y a un lien de dépendance entre la bibliothèque et la mairie, entre le bibliothécaire et le maire - qui l'a choisi et peut s'en séparer. Par rapport aux bibliothèques américaines, ce lien consubstantiel engendre deux différences fondamentales. D'une part, le dialogue est exclusif, puisque seuls deux acteurs sont sur scène. D'autre part, le contexte peut être beaucoup plus politisé, au sens partisan du terme. Ces deux éléments ont évolué au fil du temps.

Le dialogue exclusif, c'est celui qu'appelle la structure : un employeur, qui plus est bénéficiant de la légitimité que donne une élection populaire, un employé. On sait que la réalité est plus complexe, se déployant au contraire en un jeu d'acteurs multiples ${ }^{241}$ : le maire, l'adjoint à la culture, les adjoints de poids (en particulier, l'adjoint aux finances), le secrétaire général de la mairie (aujourd'hui directeur général des services), le directeur des affaires culturelles, les autres chefs de service, les directeurs des autres établissements culturels (le musée, l'opéra, les archives, etc.) - pour s'en tenir à la scène interne. Le bibliothécaire, dans cet environnement, occupe sa place, noue des alliances, porte son projet, plaide sa cause. Tout en nuançant donc mon affirmation initiale, je ne la considère cependant pas comme invalide. La décision, le pouvoir de décision, est tout entier dans les mains du maire. Qui peut la déléguer ou non, la partager ou non, être attentif aux arguments des autres acteurs ou non, être dans un calendrier électoral ou non, être préoccupé par les affaires culturelles ou non, etc., etc. Le maire, la personne du maire, ses convictions, son parcours, son projet ont indéniablement une importance beaucoup plus grande en France qu'aux États-Unis. Et de plus en plus : la fonction exécutive a pris un poids considérable au fil des trente dernières années, au fur et à mesure que les villes acquéraient du pouvoir (politique et économique), que le champ des politiques municipales s'étendait et que la politique municipale s'identifiait à la personne du maire. Le maire en son royaume, pourrait-on résumer à en croire Albert Mabileau qui évoque «la monarchie municipale à la française ${ }^{242} »$. Face à ce pouvoir fort, le bibliothécaire français est sans contexte beaucoup plus fragile, car plus isolé, que son collègue américain. 
L'autre grande différence est la politisation de la scène. Ce constat est, lui, de moins en moins avéré - mais n'en marque pas moins une différence sensible avec la situation américaine. Les élections municipales étaient politiques, elles le sont de moins en moins. Ou, plus exactement, les élus sont de plus en plus clairement identifiés à une préférence partisane ${ }^{243}$ mais les programmes des élus et les choix des électeurs sont de moins en moins politiques - au sens partisan. C'est l'avenir de la ville qui est en question, avec la qualité de vie qu'elle sera capable d'offrir à ses habitants. Sur ce registre, c'est la compétence qui est mise en avant, non une étiquette politique. « De plus en plus de maires redéfinissent pour partie leur rôle autour des notions d'efficacité, de rentabilité et de performance socio-économiques », écrit ainsi Alain Faure ${ }^{244}$. Cette évolution n'est ni achevée ni univoque. Les élections municipales sont, en effet, toujours considérées comme un bon baromètre de la situation politique : qu'on pense aux élections de 1977, gagnées par l'Union de la gauche, ou à celles de 1983 qui marquaient le réveil de la droite ; qu'on pense aux victoires symboliques qu'ont été l'élection de Bertrand Delanoë à Paris ou Gérard Collomb à Lyon (2001). Qu'on pense au coup de tonnerre qu'a été l'élection de listes du Front national dans trois communes en 1995. Occasion violente de reconsidérer la nature politique du pouvoir des élus, eux qui prônaient la guerre culturelle, eux qui faisaient dire au responsable d'une de leurs bibliothèques : « La médiathèque doit être la vitrine idéologique de la municipalité. »

Ces deux caractéristiques, un maire fort, une mairie politique, rendent plus visibles la faiblesse de la place de la bibliothèque municipale dans le système politique local. Faiblesse d'autant plus grande qu'elle n'a pas d'alliés.

\section{Les Library Boards}

Le Library Board est-il un allié ? Sans doute : à la fois décideur et défendeur, il est en tout cas le porte-parole des intérêts des Public Libraries. Avant d'examiner son rôle, quelques mots sur son identité.

Le mode d'administration des Public Libraries peut prendre trois formes : soit celle d'un service municipal intégré, comme le sont les bibliothèques

243. Dans la France urbaine, les listes « hors partis » ont disparu. Sur ce point, Élisabeth Dupoirier, Gérard Grunberg, Béatrice Roy. « L'évolution électorale de la France urbaine (1971-1983) ». Revue française de science politique, $\mathrm{n}^{\circ} 1,1985$.

244. Alain Faure. "Pouvoir local en France : la management mayoral à l'assaut du clientélisme », Politiques et management public, $n^{\circ} 3,1991$. 
municipales en France ; soit celle d'une association ; soit, la plus courante, celle d'un service public administré par un « conseil d'administration », traduction imparfaite pour Board, expression que je me garderai désormais de traduire. Ces Boards sont, la plupart du temps, spécifiques à la bibliothèque : ce sont des Library Boards ; dans le cas, très minoritaire (4\%), des bibliothèques rattachées à un district scolaire, le Board est le même que celui de l'école (School Board). C'est le Library Board classique, ordinaire, que je vais évoquer ici.

Son origine, dès la Boston Public Library, on s'en souvient, se trouve dans la volonté des promoteurs de la bibliothèque de la confier aux pouvoirs publics sans en faire un outil politique, partisan, ni priver ses usagers des moyens de contrôler son activité. En somme, il s'agissait à la fois de mettre la Public Library à l'abri de la « sale politique » et de garder l'esprit participatif de l'association d'origine. La « sale politique » ? De nombreux analystes soulignent la difficulté des bibliothécaires à admettre que la bibliothèque, institution démocratique et généreuse, est liée au processus politique. Du côté des bibliothécaires américains, la conjonction avec la sphère politique a souvent été considérée avec répugnance : «Pour certains bibliothécaires, l’idée que le développement des Public Libraries est lié en quoi que ce soit à la politique est sacrilège », écrit Edwin Beckerman ${ }^{245}$. Cette méfiance est largement partagée depuis le $\mathrm{XIX}^{\mathrm{e}}$ siècle et un certain nombre de services publics (à commencer par les écoles) sont administrés de façon autonome " pour isoler certains services municipaux de la politique et des politiciens présumés vénaux. ${ }^{246}$ » L'esprit participatif ? Il s'agit de prolonger l'idée que la bibliothèque est la mise en commun de ressources, au bénéfice de tous, et que les besoins de tous doivent donc pouvoir s'exprimer - et que tous doivent pouvoir participer, y compris par des financements privés. Protéger et participer sont donc les maîtres mots qui ont guidé la création des Library Boards. Ils sont l'émanation de ce que Phyllis Dain appelle « le mélange pragmatique de contrôle et de financement publics et privés ».

245. Edwin Beckerman. Politics and the American Public Library, op. cit., p. 23. Oliver Garceau cite des bibliothécaires qui « se sentiraient nus et sans défense s'ils devaient aller seuls à la mairie ou devant les administrateurs du comté » (Oliver Garceau. The Public Library in the Political Process, op. cit., p. 54)

246. Phyllis Dain. "American Public Libraries and the Third Sector: Historical Reflections and Implications". Libraries \& Culture, Winter 1996. Elle parle du "total disdain for the political process » qui animait les bibliothécaires pendant tout le XIX siècle. Et James Ubel souligne : « Il y a une idée presque universelle chez les bibliothécaires qui est que la Public Library doit être protégée de la politique par un puissant Library Board - le Board devant agir comme un tampon entre la bibliothèque et la mairie. » (James A. Ubel. "Library Board Forms", op. cit.) 
Le Library Board est généralement désigné par le maire et le conseil municipal, plus rarement élu par les citoyens. L'étendue de son pouvoir, vis-à-vis de la mairie, est définie, dans chaque état, par la State Library Law - en particulier sur le point de savoir qui décide les recettes (l'impôt, soit levé spécialement pour la bibliothèque, soit une part des recettes fiscales globales), et les dépenses. Usuellement, c'est la mairie qui détermine le budget, sur proposition du Board, et le Board qui engage les dépenses. Le Board recrute le directeur de la bibliothèque, décide du montant des salaires du personnel, éventuellement licencie, décide les modes de fonctionnement (horaires d'ouverture, création de services, montant des adhésions ou amendes, participation à des réseaux), programme le développement (création de nouvelles bibliothèques, construction), analyse les résultats. Il est le porte-parole des besoins de la communauté desservie auprès du bibliothécaire comme il est le porteparole des besoins de la bibliothèque auprès des pouvoirs locaux.

Vis-à-vis de la bibliothèque, l'étendue du pouvoir du Library Board est claire : la politique de la bibliothèque est du ressort du Board, la gestion de la bibliothèque de celui du bibliothécaire ${ }^{247}$. Sauf que, disent en chœur tous les analystes, cette formule ne définit rien. Les relations sont fonction de la personnalité des uns et des autres, de l'idée qu'ils se font de leur propre rôle et de leur poids réciproque. Un bibliothécaire débutant, timoré ou proche de la retraite pourra accepter sans problème un Board puissant, voire envahissant. Dans d'autres cas, un bibliothécaire mieux assis pourra " gérer » son Board ${ }^{248}$, avoir avec lui des relations constructives et confiantes : dans l'ensemble, les Boards et les bibliothécaires ont appris à travailler ensemble. Même s'il s'agit d'une institution souvent considérée comme archaïque, nul n'envisage sérieusement aujourd'hui sa suppression ${ }^{249}$. Cependant, les motifs de friction ne manquent pas, en particulier sur le choix des acquisitions, j'y reviendrai.

247. Oliver Garceau cite la définition que donne l'ALA : « Le Board décide de la politique. Le bibliothécaire a le plein contrôle de la gestion et prend toutes les décisions professionnelles. » Il commente : «It definites nothing. » (Oliver Garceau. The Public Library in the Political Process, op. cit., p. 107)

248. Oliver Garceau encourage les bibliothécaires à « garder toujours un œil sur eux, les tenir occupés, leur rendre la vie agréable » (keep them busy, keep them happy).

249. Virginia Young, qui fut présidente de l'association des membres des Library Boards (American Library Trustee Association), une des branches de l'ALA, dit fièrement : «Qu'ils soient grands ou petits, forts ou faibles, efficaces ou incompétents, ce sont eux qui gèrent les Public Libraries américaines » (Virginia G. Young. “Library Governance by Citizen Boards”. Library Trends, vol. 26, $\mathrm{n}^{\circ} 2$, Fall 1977) 
Autre sujet de réticence de la part des bibliothécaires : le Library Board, supposé représenter la population, ses besoins, ses attentes, n'est en rien représentatif. Une abondante littérature fait le constat répétitif que les membres des Library Boards sont plutôt des hommes, des Blancs, des personnes d'un certain âge et des membres des classes supérieures. Ils ne sont représentatifs ni de la population, ni des usagers. Jane Robbins dit plaisamment qu'ils « représentent plutôt les élus de la ville ou du comté qui les ont désignés que les citoyens de ces collectivités. » Dans l'enquête qu'elle a réalisée en 1972 auprès d'un échantillon représentatif de 165 Public Libraries, elle constate, par exemple, que les administrateurs représentent à 91 \% le profil social traditionnel des Library Boards (cadres supérieurs, professions libérales et femmes au foyer), que les deux tiers des Boards sont uniquement composés de Blancs (alors que seulement $14 \%$ des bibliothèques de l'échantillon ont une population uniquement blanche), que $99 \%$ des administrateurs ont plus de 40 ans et que $76 \%$ d'entre eux sont des hommes ${ }^{250}$.

Pourtant, l'idée que le Library Board représente la population est encore très vivace. Ses membres sont souvent désignés, entre autres, pour cette raison - mais pas mécaniquement et nullement sous un quelconque régime de quotas. Plutôt, grossièrement, en fonction de l'idée que l'on se fait des usagers des bibliothèques : ainsi, les femmes au foyer sont supposées représenter les intérêts des enfants ou les enseignants représenter les jeunes scolarisés. D’autres critères de choix sont la reconnaissance (pour un don fait à la bibliothèque - ou au parti du maire), la notabilité (par exemple, un responsable de la Chambre de commerce ou un membre du clergé) ou des compétences précieuses pour la bibliothèque (en matière d'emprunts, de bâtiments ou de relations publiques, par exemple) ${ }^{251}$.

Ainsi, si l'on parle de représentativité, il conviendrait d'en parler sous l'angle de la capacité à représenter, à prévoir, à plaider, à défendre, à argumenter - plutôt que sous l'angle d'une hypothétique proximité entre la population desservie et le Board. En bref, le Board représente davantage la bibliothèque auprès des autorités et de la population que la popu-

250. Jane Robbins. Citizen Participation and Public Library Policy, The Scarecrow Press, 1975. À titre d'exemple, le Library Board de la Chicago Public Library est composé, en 1968, de neuf membres : un ingénieur, un pasteur, un syndicaliste, un opticien, un journaliste, un professeur d'université, un bibliophile (spécialiste de l'époque de Lincoln) et deux femmes dont on sait seulement qu'elles sont des militantes associatives; soit sept Blancs et deux Noirs (Lowell Martin. Library Response to Urban Change: A Study of the Chicago Public Library. ALA, 1969).

251. James A. Ubel. "Library Board Forms", op. cit. 
lation auprès de la bibliothèque. Une des raisons en est, peut-être, l'importance que tient, dans ses activités, la recherche de financement.

\section{Le financement local}

" La première responsabilité des Library Boards est de veiller à ce que la bibliothèque soit convenablement financée », écrit clairement Virginia Young ${ }^{252}$. Car le financement d'origine locale est très majoritaire. Aux États-Unis, même s'il varie, évidemment, au cours du temps et selon les états et les villes, il représente généralement entre 80 et $90 \%$ du budget de fonctionnement, soit 75 à $85 \%$ de budget public local et 5 à $10 \%$ de dons ou participations des usagers. L'aide de l'état et l'aide fédérale représentent ainsi entre 10 et $20 \%$ de ce budget ${ }^{253}$. En France, entre 1969 et 1989 , le budget de fonctionnement a toujours été alimenté à plus de $90 \%$ par les villes - à l'exception des années 1982 et 1983 où l'augmentation spectaculaire du budget du ministère de la Culture a permis une progression importante, mais éphémère, des subventions de fonctionnement ${ }^{254}$. Dans les années 1990, les dotations de l'État s'élèvent à moins de $5 \%$ des dépenses de fonctionnement des villes pour leurs bibliothèques.

La source locale de financement est, on le voit, capitale. Obtenir un bon budget (en France et aux États-Unis), décider les contribuables à faire un effort (aux États-Unis) sont donc des préoccupations primordiales. Or, on s'en doute, l'organisation actuelle et l'histoire font que les bibliothèques municipales et les Public Libraries n'ont pas les mêmes atouts dans ce jeu. Deux différences essentielles dans le plaidoyer auprès des décideurs locaux : la présentation du bilan d'une part, les acteurs concernés d'autre part. Et un point commun, lui aussi, essentiel : ici, comme là-bas, les bibliothèques sont en concurrence avec d'autres services publics dans l'affectation des budgets publics. Ce qu'on dépense pour la bibliothèque, on ne le dépense pas pour d'autres. Il s'agit donc non seulement d'être convaincant dans la présentation de ses besoins (ses demandes budgétaires) mais de l'être plus que d'autres.

252. Virginia G. Young. "Library Governance by Citizen Boards”, op. cit.

253. En 1977, les ressources venaient en moyenne du budget public local pour $75 \%$, de l'État pour $7 \%$, du niveau fédéral pour $8 \%$ et des ressources propres pour $10 \%$ (Donald Sager, Managing the Public Library, op. cit.). À Chicago, en 1968, l'impôt local produisait $83 \%$ du budget, l'aide de l'état $16 \%$ et les ressources propres $1 \%$ (Lowell Martin, Library Response to Urban Change, op. cit.).

254. Anne-Marie Bertrand. Les villes et leurs bibliothèques, op. cit, p. 114. En 1982, la part municipale descendait à 78,7 \% des dépenses, en 1983, 82,5\%. Dès 1985, la jauge revenait à $90 \%$. 
En France, comme service municipal, la bibliothèque municipale (le directeur de la bibliothèque municipale) présente ses demandes à travers les échelons administratifs et politiques dont il dépend (direction des affaires culturelles, direction générale, adjoint à la culture, maire). Son discours est d'autant plus audible qu'il est lui-même reconnu et que les services rendus par la bibliothèque sont reconnus. Las, sauf exception, le bilan de l'activité est succinct : le nombre d'inscrits, le nombre de prêts restent les éléments les plus usuels. L'évaluation est toujours balbutiante et l'implicite sur les objectifs limite les arguments mobilisables.

Aux États-Unis, où les missions sont plus explicites, la liste des arguments est plus longue : le nombre de documents prêtés mais aussi le nombre d'entrées à la bibliothèque, le nombre de documents ayant circulé dans le prêt entre bibliothèques ou le nombre de questions posées par les usagers : la fonction éducative est, reste un élément essentiel ${ }^{255}$. Mais la différence majeure tient au dispositif. Si c'est, comme en France, le pouvoir politique local qui décide des ressources allouées, les demandes sont présentées par ou avec le Library Board. Par ailleurs, des auditions (hearings) peuvent être organisées, où des usagers convaincants peuvent servir d'avocats à la cause de la bibliothèque : « La présentation du budget implique des arguments raisonnablement convaincants en faveur des dépenses liées aux objectifs présentés. Cela suppose un soutien public aux programmes proposés. Bien sûr, un tel soutien n'est jamais une certitude, mais son existence devrait être évidente. Cette évidence peut être statistique, sous forme d'enquêtes ou autres, ou sous forme de témoignages personnels lors des auditions publiques ultérieures. ${ }^{256}$ »

Ce soutien public (Public support) étant un élément essentiel, sans doute le plus important, de différence entre les bibliothèques municipales et les Public Libraries, il convient de le traiter en détail.

255. Une enquête menée sous la direction de George D’Elia en 1992 montre que le public plébiscite le(s) rôle(s) éducatif(s) de la Public Library : est considéré comme " très important » le rôle de la bibliothèque (dans l'ordre) comme soutien éducatif pour les étudiants (88\%), comme centre de ressources pour les adultes en formation (independent learners, $85 \%$ ) et comme centre de découverte pour les enfants d'âge pré-scolaire (83 \%) (George D'Elia. The Roles of the Public Library in Society: The Results of a National Survey. Final Report. Evanston, Urban Libraries Council, 1993). George D’Elia note que, comme dans la plupart des enquêtes de pratiques culturelles, il y a un écart entre ce que les gens disent apprécier, estimer (value) (la bibliothèque comme ressource éducative, au sens large) et ce qu'ils utilisent (use) réellement (l'emprunt de documents principalement, des fictions, popular materials).

256. Edwin Beckerman. Politics and the American Public Library, op. cit., p. 40. Ainsi, en mai 2002, pour expliquer les dommages que pourraient causer les coupes budgétaires envisagées pour la New York Public Library, un petit Croate de 10 ans vient témoigner devant le Conseil que, jeune immigrant, il avait appris l'anglais grâce à la bibliothèque (American Libraries on line, posté le 3 juin 2002). 


\section{AMIS, ALLIÉS ET SOUTIENS}

Les deux caractéristiques principales dont se réclament les Public Libraries, leur visée démocratique, leur enracinement local, appellent et engendrent un soutien public. Comme en France, ce soutien existe à l'état latent : les Français aiment leurs bibliothèques municipales, les Américains aiment leurs Public Libraries. En France, une enquête menée en 1997 par IPSOS montrait une estime générale, une reconnaissance du rôle joué par les bibliothèques ${ }^{257}$. Aux États-Unis, régulièrement, les sondages et les enquêtes montrent cette même estime. Comment la transformer en soutien actif ? En France, les bibliothèques souffrent à la fois d'une vie associative faible et de leur origine aristocratique, soulignée à maintes reprises par Eugène Morel : « On peut dire qu'en France les bibliothèques viennent de haut. Elles sont comme les officiers, d'une autre race que les soldats : elles ne se mêlent pas au public. ${ }^{258}$ " Cette origine si peu démocratique a pénalisé leur développement depuis le XIX siècle, comme l'a si bien montré Jean Hassenforder, qui écrit notamment : « La bibliothèque publique est une institution destinée à tous. Son développement suppose, en conséquence, un assentiment de la part d'une majorité au moins de la population. Il exige un intérêt partagé, la conscience d'un bien commun. Les tensions propres à la société française à la fin $\mathrm{du}_{\mathrm{XIX}}^{\mathrm{e}}$ siècle, se révèlent défavorables à la bibliothèque publique. Alors qu'aux États-Unis et en GrandeBretagne, le mouvement en faveur des bibliothèques recrute des partisans dans l'ensemble des milieux sociaux, en France, ces assises se révèlent beaucoup plus restreintes. ${ }^{259}$ »

\section{Des soutiens organisés}

L'histoire des bibliothèques mais aussi la vie politique et la vie sociale aux États-Unis permettent plus facilement ce passage de l'estime (abstraite) à l'implication (concrète).

\footnotetext{
257. Anne-Marie Bertrand, Martine Burgos, Claude Poissenot, Jean-Marie Privat. Les bibliothèques municipales et leurs publics. BPI, 2001. $93 \%$ des usagers des bibliothèques municipales et $75 \%$ des non-usagers considèrent que la bibliothèque est « utile à tous » (p. 64).

258. Eugène Morel. La librairie publique, op. cit., p. 7.

259. Il écrit aussi : "Si la France se caractérise par la moindre intensité de la vie d'association et la faiblesse relative du gouvernement local, ce sont là deux facteurs conjugués qui ne favorisent pas la prise de conscience des besoins réels de la population et la traduction de cette prise de conscience dans les faits par la mise en œuvre de réalisations concrètes destinées à répondre à ces besoins ». Jean Hassenforder, Développement comparé des bibliothèques publiques en France, en GrandeBretagne et aux États-Unis, op. cit., p. 105.
} 
En France, la faiblesse des associations est fréquemment soulignée. Elle est due, on l'a souvent dit, à la centralisation du pouvoir (central ou local), ou à une tradition intériorisée d'un pouvoir éloigné qui rend à peu près inopérant le pouvoir d'influence des citoyens ou des groupes constitués ${ }^{260}$. Observateur avisé, Stanley Hoffmann a jadis souligné les spécificités françaises de ce point de vue : «Au lieu de résoudre des conflits par la coopération et le compromis, les individus et les groupes renvoient ces conflits à une autorité supérieure et centrale, ainsi rendue responsable du résultat. Cette attitude conduit tout d'abord à la centralisation (dans les entreprises et les administrations, comme dans l'ensemble du système politique) ; en second lieu, à la fragilité des associations volontaires auxquelles l'individu appartient - leur fonction est essentiellement de le protéger contre un arbitraire possible d'en haut et de promouvoir ses intérêts “publics”, tout en évitant d'empiéter sur son indépendance en tant qu'individu privé. ${ }^{261}$ » Pour une période plus contemporaine, Pascal Viot, en rappelant la faible appartenance syndicale ou l'affaiblissement des associations de parents d'élèves, souligne un déplacement des formes d'adhésion volontaire : "Avec la fin des grands discours idéologiques, il y a un changement d'horizon ; il semble qu'il s'agit moins aujourd'hui, dans les associations, de se battre pour des lendemains meilleurs que davantage se prémunir ou de conjurer de nouveaux risques envahissants (la pauvreté, le saccage de la planète, les conflits, la globalisation). ${ }^{262}$ »

Loin d'être conçues pour peser dans des conflits, les associations d'amis des bibliothèques, en France, sont souvent des associations créées, voulues et pilotées par les bibliothécaires pour leur fournir un peu de souplesse dans la gestion financière de leur établissement - souplesse concernant ici plutôt la procédure (payer des factures vite, ou par avance) qu'un apport financier. Ces associations sont ainsi, par nature, proches de la direction de la bibliothèque et nullement représentatives des usagers. Elles aident la bibliothèque à enrichir leurs collections, à monter des événements mais non (ou très peu) à nouer des relations avec son environnement.

260. Je parle ici non pas des lobbys (industrialo-militaires, agro-alimentaires, pharmaceutiques, etc.) mais des associations ou groupes locaux, tels les associations de parents d'élèves ou les amis de la bibliothèque.

261. Stanley Hoffmann. Essais sur la France : déclin ou renouveau ? Éditions du Seuil, 1974, p. 173. Il ajoute que la France, « une nation qui a traditionnellement accepté l'idée, développée sous l'Ancien Régime et reprise par la Révolution, que seul l'État représente l'intérêt général, continue à considérer les groupes privés avec suspicion », p. 503.

262. Pascal Viot. " Enjeux et perspectives de l'engagement associatif », Lecture publique et territoires : trente ans de mutations en BDP. Presses de l'enssib, 2005. 
Comme son nom l'indique, une autre institution, les comités d'usagers, a pour fonction de permettre le dialogue entre la bibliothèque et ses publics. Elle existe très rarement, car les bibliothécaires éprouvent une méfiance farouche vis-à-vis de la parole de " profanes » sur leur activité professionnelle. Fréquente, voire habituelle, dans les administrations, cette défiance des fonctionnaires vis-à-vis des usagers relève de l'idée que les fonctionnaires sont détenteurs de l'intérêt général - et non les usagers, immédiatement soupçonnés de défendre des intérêts privés, ni d'ailleurs les élus soupçonnés, eux, de défendre des intérêts partisans ${ }^{263}$. Des " comités consultatifs », essentiellement composés de notables, ont été institués par décret en 1961 et sont peu à peu tombés en désuétude, tant leur composition était inadaptée.

Des re-créations ou tentatives de renaissance de ces comités ont lieu régulièrement sous une autre forme et un autre nom. Quelques municipalités en ont mis en place. Déjà peu enclins à les accueillir favorablement, les bibliothécaires sont aujourd'hui (depuis l'agression du Front national) encore plus réticents : alors que leur seule défense dans cet épisode a été corporatiste (c'est leur professionnalisme, dirent-ils, qui doit leur garantir l'exclusivité des politiques d'acquisitions), l'implication de non-professionnels dans la marche de l'établissement est plus que jamais vécue comme une intrusion injustifiée. Chroniqueur dans le Bulletin d'informations de l'ABF, Hugues Van Besien nous fournit une illustration de cette méfiance : « Découvrant qu'au fond elles ne connaissaient rien à la façon dont s'effectuaient les acquisitions à propos des affaires des bibliothèques gérées par des municipalités FN, des collectivités assez nombreuses ont pensé à mettre en place ce type de conseil par précaution, et sans voir à quels risques de noyautage, ou simplement de confiscation, elles s'exposaient, à une époque où des militants politiques vérifiaient liste en main le respect de leur conception du pluralisme dans les bibliothèques [...]. Mémoire et prudence s'imposent donc. ${ }^{264}$ » Il ajoute, cependant, que, par leur appui, «nos usagers peuvent devenir notre force ». Vœu pieux, ou du moins isolé, tant c'est la méfiance qui prime.

263. « L'administration est porteuse de l'intérêt général, à la fois parce qu'elle est subordonnée aux représentants élus de la nation, et parce qu'elle est dépositaire d'un savoir qui lui permet de s'élever au-dessus des querelles partisanes traversant la représentation politique ", écrivent François d'Arcy, Claude Gilbert et Guy Saez, « Nouveaux clivages dans les élites étatiques », Administration et politique sous la Cinquième République, Presses de la Fondation nationale des sciences politiques, 1981.

264. Hugues Van Besien. « Comités d'usagers : le retour? », Bulletin d'informations de l’ABF, n 187 , 2000. 
Jean-Luc Gautier-Gentès, inspecteur général des bibliothèques, explicite les raisons de cette méfiance : « Un comité consultatif est susceptible de présenter au moins deux dangers. Le premier est de servir de tribune à un groupe agissant pour le compte d'une idéologie (au sens large du terme) et qui se servira de lui pour tenter d'infléchir la politique d'acquisitions conformément à cette idéologie. C'est à ce danger qu'une composition véritablement plurielle est destinée à parer, les pressions des différents groupes présents étant susceptibles de se neutraliser les unes les autres. Plus insidieux, mais non moins réel, est l'autre danger : celui que les difficultés soulevées par une de ces acquisitions problématiques que nous évoquions plus haut se résolvent par l'abstention, autrement dit, dans une sorte de "consensus mou", cette abstention pouvant d'ailleurs revenir, dans certains cas, à céder aux exigences d'un groupe de pression. Prenons un exemple. Le désir de ne pas choquer une éventuelle communauté musulmane locale, ne conduirait-elle pas le comité à préconiser de ne pas acquérir Les Versets sataniques de Salman Rushdie ? ${ }^{265}$ ”

Les usagers ont peu de place dans les bibliothèques municipales, même dans les relations informelles, comme le souligne encore Jean-Luc GautierGentès : " Mais dans ce mode d'association du public à la marche de la bibliothèque, le public est cantonné au plus petit rôle possible. Quel que soit son nom, doléance ou suggestion, sa prise de parole tient peu ou prou de la supplique. L'usager propose, le bibliothécaire dispose [...]. Et si les bibliothécaires sont si peu pressés de voir des conseils consultatifs refleurir à leur côté, n'est-ce pas aussi - osons dire ce que chacun sait bien parce que, comme tout groupe professionnel, ils n'éprouvent aucun plaisir à l'idée que l'on empiète sur leur champ de compétence, autrement dit, sur le territoire où s'exerce leur pouvoir propre, réel et symbolique ?» Le sociologue présente une analyse plus distanciée : " On peut donc formuler l'hypothèse que les professionnels des bibliothèques, au sens large, qu'ils soient militants de la lecture publique ou gestionnaires pragmatiques, sont, pour la plupart d'entre eux, dépositaires à des degrés divers de valeurs anciennes qui concernent le rôle qu'ils doivent tenir et celui qu'ils réservent aux publics. ${ }^{266}$ "

Acteurs plus ou moins engagés ou praticiens méfiants, les bibliothécaires français admettent, reconnaissent cette distance, cette méfiance

265. Jean-Luc Gautier-Gentès. "Réflexions exploratoires sur le métier de directeur de bibliothèque », Bulletin des bibliothèques de France, t. 44, n 4, 1999.

266. Christophe Evans. "La place des publics dans le modèle français : une approche sociologique ». Quel modèle de bibliothèque ?, op. cit., p. 86-87. 
entre les usagers et eux. Ce constat ne va pourtant que rarement jusqu'à suggérer ou engager des changements sur ce point ${ }^{267}$. Comme si un autre type de relation, de dialogue, de considération n'était que difficilement possible - l'apparition du Web 2.0 incite à nuancer cette analyse, les bibliothécaires semblant désireux, par cet outil, de donner la parole aux usagers ; mais sur quels sujets ? Des coups de cœur, des commentaires, des suggestions d'achat. Des goûts - pas des projets, des priorités ou des arbitrages.

La situation est toute différente aux États-Unis. La Public Library, émanation populaire, a besoin de l'assentiment et du soutien populaires pour exister, pour se développer, pour être financée. La recherche permanente de ce soutien a des effets sur les services proposés ou les documents achetés par la Public Library mais aussi, bien entendu, sur sa communication, ses relations publiques, l'affirmation de son image. Au niveau national, c'est l'ALA qui mène cette politique de communication. Au niveau local, chaque bibliothèque tisse ces liens avec sa community. Depuis les années 1960, un souci majeur est d'être user-oriented : de mettre l'usager au cœur des préoccupations.

Le premier aspect est la satisfaction des usagers. Une Public Library ne peut espérer (et d'ailleurs ne mériterait pas) le soutien de sa population si elle n'était pas attentive à ses attentes et à ses demandes. Si la Urbana Free Public Library (Illinois, 43000 habitants) est si populaire, si fréquentée, c'est qu'elle "rencontre la demande des habitants », elle est « réactive et chaleureuse », un best-seller peut être acheté en 30 ou 40 exemplaires pour qu'on puisse vraiment le trouver en rayons, on répond vraiment aux questions posées par téléphone, on ne se contente pas de basculer sur un poste qui ne répond pas - et « tout le monde sourit » ${ }^{268}$. La bibliothèque, construite en 1917, avait sérieusement besoin d'être réaménagée et agrandie : le maire tergiversait, il a été battu aux élections. Sur les 8 millions de dollars qu'ont coûté les travaux, 6 millions venaient du budget municipal et 2 millions de 1200 donateurs, des citoyens de la ville. Soutien actif.

Un autre élément de cette relation positive est la gratuité des services : aujourd'hui encore très majoritaire, la gratuité est défendue comme

267. Christophe Evans ajoute : «On ne remplace pas facilement et rapidement un symbole soutenu par un système de normes et valeurs professionnelles. »

268. Entretien avec Fred Schlipf, directeur de la bibliothèque, 15 juin 2005. 
faisant partie de l'identité même de la Public Library - au reste, Public Library et Free Library sont des synonymes. Dans la plupart des cas, les seuls éléments payants sont ce qui est considéré comme excédant les services de base (les photocopies, la location d'une salle) ou les amendes pour retard. L'introduction d'Internet pour le public a suscité des questions : prise en charge partielle, totale ou nulle par les usagers ? La grande majorité des Public Libraries ont opté pour un service gratuit (mais limité dans le temps). Les arguments employés contre le paiement sont proches de ceux que l'on utilise en France (les sommes collectées sont dérisoires, la collecte du paiement coûte plus cher qu'elle ne rapporte, le budget risque d'être diminué du fait de ces recettes supplémentaires et, bien sûr, l'information est un bien public qui doit être facilement accessible) à l'exception d'un argument, propre aux Public Libraries : « la perte du soutien public si des coûts d'utilisation sont institués. Un des aspects en est le danger que, s'il y a des coûts d'utilisation, les usagers verront la Public Library comme différente, comme une institution commerciale. L'image de la bibliothèque pourrait donc être sévèrement atteinte par l'établissement de ce paiement [...]. L'identité même de la Public Library, forgée depuis plus d'un siècle en étroite relation avec la population locale, serait mise en danger par l'instauration de ce paiement. ${ }^{269}$ » Cet argument n'est pas utilisé en France, et c'est normal puisque l'« étroite relation avec la population locale » n'est pas un des éléments de l'identité de la bibliothèque.

Revenons sur les soutiens organisés et, au premier chef, sur les Library Boards. Car ils sont le premier avocat de la bibliothèque, son premier porte-parole. Ils sont le bouclier grâce auquel les bibliothécaires ne se sentent pas " nus et sans défense ». Les membres du Library Board, les Trustees, apportent à la bibliothèque leur énergie, leurs compétences, leurs relations, leur entregent, leur courage - « Ceux qui assument la responsabilité d'être des trustees ne peuvent pas être des âmes timides. Les citoyens qui acceptent le défi de gérer une bibliothèque doivent être dotés d'une ampleur de vision, de convictions profondes et du courage d'oser l'impossible. Voilà l'engagement qui est attendu des citoyens qui participent aux Library Boards. ${ }^{270}$ "Vision évidemment idyllique, mais qui a l'avantage de souligner le caractère militant de cet engagement. Cet acti-

269. John Jaeger. “User Fees, Community Goods and the Public Library”. Public Library Quarterly, $1999, n^{\circ} 2$.

270. Virginia G. Young. "Library Governance by Citizen Boards”, op. cit. 
visme positif n'est pas universellement répandu, et on cite aussi des trustees qui n'assistent pas aux réunions, ignorent tout des bibliothèques ou n'ont pour seule influence que celle de faire retirer des rayons les livres qu'ils désapprouvent. En tant qu'organisation, ils sont cependant le premier soutien des Public Libraries. Mais soutien par essence, par nature : il ne suffit pas à créer ni même à manifester le soutien de la population à sa bibliothèque.

D'autres relais sont indispensables. Le premier d'entre eux est, comme en France, les associations d'amis de la bibliothèque (Library Friends). Chargés de "tisser des liens avec la communauté », les amis de la bibliothèque sont d'abord "un groupe de soutien » : quelques personnes qui forment le noyau d'un groupe actif dont les activités (expositions, rallyes, concerts) aideront la bibliothèque à être appréciée et, incidemment, pourront engendrer des dons ${ }^{271}$ - dans les grandes et anciennes Public Libraries, cet intérêt est souvent lié à l'enrichissement d'un fonds particulier et contribue à entretenir d'excellentes relations avec les collectionneurs. Ces associations peuvent servir de relais, de chambres d'écho. Elles sont regroupées dans une association, Friends of Library USA (FOLUSA), qui regroupe plus de 2500 associations d'amis, soit plus d'un million de personnes à travers le pays. Oui, un million de personnes.

De façon moins formelle, les "volontaires » (Volunteers) apportent à la Public Library non seulement du temps de travail gratuit, mais aussi un lien avec la communauté auprès de qui ils peuvent devenir le porte-parole de la bibliothèque, la connaissant de l'intérieur. Les volontaires ne sont pas toujours bien accueillis par le personnel de la bibliothèque, qui craint évidemment que ce travail gratuit n'en vienne à remplacer leur travail payé. De la même façon, il arrive que les associations d'amis soient mal perçues - notamment par les Library Boards qui peuvent y voir des concurrents. Mais dans la grande majorité des cas, ces groupes sont considérés comme des outils précieux dans la communication de la bibliothèque et dans ses relations avec la population qu'elle doit desservir.

Prenons, à titre d'illustration, l'exemple du centenaire de la Rutherford Library (New Jersey, 18000 habitants) en 1994 : le but de cette célébration est, écrit la bibliothécaire, « d'élever le niveau de connaissance qu'a la population de notre travail et de créer un canal de communication à

271. « Dans cette vie urbaine sous pression, certains peuvent plus facilement faire un chèque que donner de leur temps », Merrily Taylor, "It's Hard to Make New Friends: What to Think About in Creating a Friends of the Library Group”, Library Trends, Winter 2000. 
travers lequel les habitants pourraient exprimer leur opinion ${ }^{272}$ ». La bibliothèque crée un comité d'organisation dont elle confie l'animation à la présidente des Amis de la bibliothèque. La première tâche est de mobiliser des volontaires : on fait appel à quelques personnes, civic-minded movers and shakers, expression évidemment intraduisible littéralement car nous n'avons pas l'équivalent en France ? Une dizaine de personnes forment le noyau initial. Elles contribuent, avec les bibliothécaires, à définir les buts de la célébration du centenaire : « renforcer les liens de la bibliothèque avec tous les secteurs de la population ; recueillir une substantielle somme d'argent », somme qui sera affectée spécifiquement à l'achat de cédéroms («pour éviter que la municipalité n'en profite pour diminuer le budget de la bibliothèque »). Des associations (en particulier l'influent Rutherford's Women's Club), des groupes informels, les écoles, le musée, des commerçants, des personnes jugées importantes (car relais d'opinion) sont peu ou prou associés à la préparation de l'événement. Une loterie, une visite des maisons centenaires de la ville, des expositions, un calendrier, une lecture-marathon (read-a-thon, entamée par le maire), une chorale, l'oblitération d'un timbre souvenir, un livre d'or, du café et, bien sûr, des cookies marquent la journée. L'opération rapporte 24000 \$. "Mais, peut-être plus important, des voies de communication ont été ouvertes entre la bibliothèque et la population et des approbateurs passifs ont été transformés en soutiens actifs ", conclut la bibliothécaire.

Car là est le cœur du problème : rendre manifeste le soutien de la population à sa Public Library. Passer de l'approbation passive au soutien actif. De l'estime à l'implication. Pour sensibiliser la population, des événements nationaux sont organisés, dont la National Library Week, des enquêtes mettent régulièrement en évidence l'impact des Public Libraries dans le pays $-84 \%$ de satisfaits, plus de livres prêtés chaque jour que de paquets transportés par FedEx, plus d'un milliard d'entrées par an (seulement 240 millions dans les stades), il y a plus de Public Libraries que de MacDonald's, etc., etc. Au-delà des campagnes de communication, un instrument de mesure efficace est l'aspect financier : les Américains apprécient leurs Public Libraries et acceptent de dépenser de l'argent pour elles. 


\section{Des soutiens actifs}

Dans une enquête menée en 2003 pour l'ALA, 63 \% des personnes interrogées disent qu'elles approuveraient peu ou prou une augmentation des impôts pour leur Public Library ${ }^{273}$.

Il ne s'agit pas que de sondages, où l'on peut prendre une pose généreuse à bon compte. De trois façons, les usagers, les contribuables, les citoyens manifestent leur engagement : soit en poursuivant la tradition philanthropique en faveur des bibliothèques ; soit en votant en faveur de l'un des referendums organisés au niveau local ou au niveau de l'état pour autoriser la création d'un impôt spécifique pour la Public Library (ou l'augmentation ponctuelle de l'impôt pour construire une bibliothèque) ; soit en intervenant comme défenseurs, avocats des services de la bibliothèque quand ils sont menacés.

Même si l'époque où les grands mécènes donnaient aux bibliothèques semble révolue (au bénéfice des musées ?), un petit courant persiste. Petit courant qui peut prendre des allures de fleuve majestueux, quand les fondateurs de Microsoft, Paul Allen et Bill Gates, donnent chacun vingt millions de dollars à la Public Library de Seattle, l'un en 1998, l'autre en 2000. Dons exceptionnels, bien sûr, mais on trouve régulièrement mention de legs ou de dons plus modestes, effectués à la bibliothèque en reconnaissance des services qu'elle a rendus : ainsi, un enseignant retraité lègue 6,8 millions de dollars à la Boston Public Library (Boston Globe, 7 décembre 2000), un couple du Kansas donne 1,2 million à la Topeka and Shawnee County Public Library (American Libraries, avril 2000) ou un couple de philanthropes de Baltimore un million de dollars à la Enoch Pratt Public Library de Baltimore pour développer la " Maryland Collection ", son fonds régional (American Libraries, janvier 2002). Un donateur anonyme dote la Public Library de Philadelphie de 15 millions de dollars, pour l'aider à meubler son bâtiment en cours de rénovation (American Libraries on line, posté le 20 avril 2007). Les grandes bibliothèques sont, bien sûr, les mieux placées pour attirer les donateurs.

S’il y a des périodes plus ou moins fastes (plus ou moins marquées par la Tax Revolt, l'opposition à l'impôt), les referendums instituant des dépenses en faveur des bibliothèques sont majoritairement gagnés : depuis une

\footnotetext{
273. "Support or strongly support increasing taxes for Public Library services in their community", "The Public Library: A National Survey", sur le site de l'ALA :

< http://www.ala.org/ala/pio/piopresskits/2004placonferencepresskit/004PublicLibraryNatio.htm >.
} 
vingtaine d'années dans une proportion des deux tiers ou des trois quarts. En 1987, année " euphorique », $93 \%$ des referendums pour des constructions sont gagnés ; en 1988, $64 \%{ }^{274}$. En 1988, les 45 referendums organisés à travers tout le pays représentaient un montant total de 138,5 millions de dollars : 29 ont été gagnés, pour un total de 104 millions, dont un gros projet de 55 millions pour la Phoenix Public Library (Arizona), remporté par $58 \%$ des votes. Entre 1987 et 1991, 245 referendums sont organisés à travers le pays : $80 \%$ d'entre eux sont approuvés avec une moyenne de $64 \%$ de votes favorables ${ }^{275}$. En 2003, 59 des 89 referendums organisés (soit les deux tiers) sont approuvés ; en 2004, 82 sur 133 (soit $61 \%$ ). L'importance des sommes en jeu (sous forme d'un emprunt dont le remboursement augmentera les impôts) ne semble pas être rédhibitoire : de gros projets comme Seattle (193 millions de dollars, en 1998) ou Minneapolis (140 millions, en 2000) sont adoptés facilement. Un travail de terrain, de conviction, de pédagogie est nécessaire pour entraîner l'adhésion des contribuables.

À Seattle, la directrice de la Public Library explique ainsi que le vote a été positif parce que ce travail de communication a été fait : " On a parlé aux gens, on les a écoutés, on a changé le projet pour tenir compte de ce qu'ils disaient » (American Libraries, décembre 1998). À Deerfield (Illinois), exemple inverse, un projet de 25 millions de dollars a été nettement repoussé (par $69 \%$ des votes) : « Je pense que les bibliothécaires fournissent un service si bon que la population ne s'est pas vraiment rendue compte des insuffisances du bâtiment actuel », commente un des administrateurs de la Deerfield Public Library (American Libraries, janvier 2005).

Enfin, dernier mode d'intervention des usagers : la défense de la Public Library en cas de besoin. Les crises budgétaires que traversent régulièrement les Public Libraries (tous les dix ans, disent les observateurs désenchantés) donnent l'occasion aux usagers de se mobiliser. La presse professionnelle fait largement écho à ces manifestations de soutien, si bien que l'on peut se demander si elles sont d'autant plus soulignées qu'elles sont rares. Sans préjuger, donc, de leur fréquence (qui n’est mesurée nulle part), il convient de les mentionner - d'autant plus qu'elles sont, elles aussi, un élément de différenciation d'avec les bibliothèques munici-

274. Richard B. Hall. “Referenda for Public Library Buildings 1988”, Library Journal, 15 June 1989. Richard Hall signale que les données étant déclaratives, elles ne sont pas forcément exactes, certaines bibliothèques pouvant s'abstenir de faire part de leur échec.

275. Richard B. Hall. Financing Public Library Buildings. Neal-Schuman Publishers, 1994, p. 190. 
pales françaises. Le scénario est simple : les pouvoirs publics annoncent que le prochain budget de la bibliothèque sera en baisse ; les bibliothécaires expliquent à la population ce que cela voudra dire en termes de services rendus (réduction des horaires d'ouverture, fermeture de bibliothèques de quartier, licenciement de personnel, etc.) ; les citoyens protestent ; souvent (mais pas toujours), les élus reviennent partiellement ou totalement sur leur décision.

Ainsi, à la Mansfield/Richland County Public Library (Ohio), où l'état avait annoncé la suppression pure et simple de l'aide aux Public Libraries, «notre population a réagi par des coups de téléphone, des e-mails et des lettres à leurs députés. Nous savons qu'ils ont entendu ces protestations, puisqu'ils ont modifié le budget. » (Public Libraries, septembre-octobre 2003). En juin 2003, les administrateurs du Los Angeles County (Californie) redonnent à la County Library les 7 millions de dollars dont ils voulaient amputer son budget, après avoir été inondés (flooded) d'appels de soutien de la part des habitants ${ }^{276}$. Le gouverneur du Wisconsin a renoncé en 2003 à proposer de nouvelles diminutions de budget pour la State Library, après l'avalanche de protestations de l'année précédente (deluged by the public).

La Cincinnati Public Library annonce le 16 juillet 2002 que la diminution de son budget va l'amener à fermer 5 de ses 41 bibliothèques de quartier, dont celle de Greenhill. Le lendemain, 17 juillet, 70 personnes se réunissent à la bibliothèque de Greenhill pour protester et témoigner de leur attachement : " La bibliothèque est le bâtiment le plus important de cette ville ", déclare une habitante. Lors d'une audition avec le Library Board, en octobre, 400 personnes viennent protester. En novembre, le Library Board renonce à la fermeture des bibliothèques car « cette idée a rencontré l'opposition des habitants ${ }^{27}$ ".

D'autres modes d'intervention sont plus inventifs : ainsi les concessionnaires automobiles de Ventura ont réuni les 67000 dollars qui manquaient à la Ventura County Library pour garder ouvert son centre d'aide aux devoirs. "Si notre don permet à un seul enfant de recevoir une meilleure éducation, cela lui donnera toute sa valeur », dit Bob Gregory, président de Paradise Chevrolet ${ }^{278}$. Peut-être moins étonnant pour nous, une soirée de gala à Hollywood, à la Saint-Sylvestre 1999, a rapporté 2 mil-

276. "Recession 2003: More Cutbacks and Closures”, American Libraries on line.

277. Postés les 22 juillet, 28 octobre et 18 novembre 2002, American Libraries on line.

278. "Recession 2003: More Cutbacks and Closures", American Libraries on line. 
lions de dollars à la Los Angeles Public Library. " Moins étonnant ", si l'on considère qu'il est étonnant que des concessionnaires automobiles s'intéressent assez aux bibliothèques pour mettre la main à la poche : on peut dire qu'en France c'est un geste inusité.

Le pouvoir d'influence des citoyens peut, évidemment, jouer aussi dans l'autre sens, contre les projets de la Public Library. On en cite quelques exemples, mais qui finissent bien : après avoir protesté contre un projet d'implantation, les habitants approuvent un autre projet ; après avoir voté contre un referendum augmentant les ressources de la bibliothèque, ils votent pour l'année suivante. Par contre, une position de principe intangible est celle des militants anti-impôts (anti-tax crusaders). Un exemple est celui, en 2002, d'un comté de l'état de Washington, le Stevens County : lassés de payer pour un service qu'ils n'utilisent pas, outrés par le salaire de la bibliothécaire, convaincus qu'Internet rend obsolète l'idée même de bibliothèque, ils réunissent assez de signatures pour faire organiser un referendum sur la fermeture de la bibliothèque du comté ${ }^{279}$. Un exemple proche, celui de l'état de Californie : le referendum, organisé en juin 2006 et destiné à financer à hauteur de 600 millions de dollars les projets de la State Library a été repoussé par $53 \%$ des votants - un habitant de Palo Alto commente : «Il faut en finir avec ces dépenses déraisonnables. Internet a changé le monde et les bibliothèques sont beaucoup moins utiles aujourd'hui qu'avant» (American Libraries on line, posté le 9 juin 2006). En l'absence de statistiques nationales, il est difficile de dire si ce cas de figure est fréquent.

En tout cas, en positif ou en négatif, l'implication des citoyens/contribuables est réelle. Les modes d'intervention le leur permettent : en France, nul referendum ne permet de compter ses partisans. Mais il serait loisible qu'ils interviennent auprès des décideurs, ce qui semble être très rarement le cas ${ }^{280}$. Par ailleurs, j'y reviendrai, les attaques contre les bibliothèques municipales de Marignane, d'Orange ou de Vitrolles n'ont pas suscité de mobilisation populaire.

On peut en tirer deux enseignements complémentaires. Le premier est que rien n'est fait, en France, pour que les habitants, les usagers, les

279. Posté le 26 août 2002, American Libraries on line.

280. Dans ma recherche sur la décision de construire une bibliothèque, j'ai montré que des influences pouvaient s'exercer soit par le biais des amis de la bibliothèque, soit par celui des enseignants, soit par celui de militants politiques. Mais les cas semblent rares et ne concernent pas l'expression des citoyens ordinaires (Anne-Marie Bertrand, Les villes et leurs bibliothèques, op. cit). 
citoyens, les contribuables, s'expriment sur ce qu'ils attendent de leur bibliothèque et sur le prix qu'ils sont prêts à y mettre. Le second est que si rien n'est fait et si tout le monde semble trouver cette absence normale, c'est que les bibliothèques ne participent pas réellement de la vie démocratique locale. Au-delà de la rhétorique habituelle, force est de constater que, en France, les bibliothèques publiques ne sont pas un sujet qui concerne les citoyens. Dans les deux sens : cela ne les regarde pas et ils ne se sentent pas concernés. Un océan nous sépare bien des ÉtatsUnis. 
CHAPITRE IV GROUPES PROFESSIONNELS ET ASSOCIATIONS 


\section{CHAPITRE IV GROUPES PROFESSIONNELS ET ASSOCIATIONS}

$1 \begin{aligned} & \text { près avoir à plusieurs reprises évoqué les bibliothécaires améri- } \\ & \text { cains et leur association, l'ALA, il convient sans doute d'y revenir } \\ & \text { plus complètement et de faire, là aussi, une analyse comparée }\end{aligned}$ entre les deux pays.

Une fois encore, prenons la précaution de souligner l'écart structurel entre nos deux pays : la culture associative, l'engagement public, le nombre des bibliothécaires, leur poids collectif ont peu à voir d'un pays à l'autre. S’il ne s'agit pas, ici, de comparer les environnements ou les contextes, il est cependant éclairant d'analyser les écarts dans les organisations, les activités, les investissements - ce qui a été choisi, construit, par les groupes professionnels, ces acteurs privilégiés de la vie des bibliothèques. J'envisagerai ici deux registres. D'une part, elles servent de porte-parole aux bibliothécaires, aux bibliothèques et aux usagers des bibliothèques, elles les promeuvent, les défendent, les rendent visibles et audibles : elles sont des interlocuteurs naturels des pouvoirs publics et de l'opinion publique. D'autre part, elles ont mené, pendant ce demi-siècle, des combats qui sont significatifs de ce qui, au-delà des discours, est effectivement jugé comme important par elles : ce pour quoi (ou contre quoi) on se bat, c'est ce qui compte ${ }^{281}$.

\section{DES ORGANISATIONS}

Pour entamer cette analyse, présentons les associations (l'ALA et, pour la France, l'ABF qui est encore quasiment monopolistique pour la période qui nous intéresse ici). Leur constitution, leur organisation, le rôle qu'elles se donnent sont significatifs. 


\section{Un peu d'histoire}

\section{The American Library Association}

Difficile de parler sans emphase de « la plus vieille et la plus importante association de bibliothécaires dans le monde ", comme elle aime à se définir. Fondée en 1876, the American Library Association (ALA) comptait, en 2008, 66624 membres et était organisée en 11 divisions (par type de bibliothèque), 17 commissions (round tables, par thème), 57 organisations régionales (chapters, souvent par état) et 25 organisations affiliées, chacune d'entre elles possédant à son tour des sections, comités et autres groupes de travail - les observateurs estiment à environ 1200 le nombre des entités qui constituent l'ALA. Elle est administrée par un Conseil d'administration (Council), qui compte 182 membres, et gérée par un Bureau exécutif de 12 personnes. Le personnel salarié de l'ALA, en 2003, comptait 270 personnes, sous la responsabilité d'un directeur exécutif. Cette multiplicité de composantes, aussi logique soit-elle, pose le problème de la gouvernance de l'association et du poids réel que peuvent avoir ses adhérents.

Le président de l'ALA est élu pour un mandat d'un an seulement ${ }^{282}$ - même si cette brièveté est balancée par l'institution du President Elect, élu un an à l'avance et qui fait fonction de vice-président avant de prendre ses fonctions de président alors que le président sortant (Immediate Past President) est, lui aussi ex officio, membre du bureau pendant encore un an. La brièveté du mandat ne fait qu'accentuer le pouvoir des cadres salariés de l'association et, au premier chef, de son directeur exécutif.

Les relations entre le président, le bureau, le Conseil, les permanents salariés et les membres de l'association connaissent souvent des périodes de tension - qui s'expriment principalement dans les rapports de fin de mandat, au moment où la parole se libère. À la conférence de 1987, la présidente sortante, Regina Minudri, écrit dans le rapport d'activité qu'elle présente au Conseil : "L'ALA est un gros animal bureaucratique (a bulky bureaucratic beast) qui semble souvent avancer à la vitesse d'un glacier [...].

282. Brièveté souvent regrettée, mais qui a pour objet d'encourager la vie démocratique de l'association. Un des anciens présidents dit : "Voilà, j'achève mon mandat, juste au moment où j’ai appris ce qu'il fallait faire et comment le faire » (Cité par Peggy Sullivan, "Library Associations", Library Trends, July 1976). La brièveté a deux objets : obliger à un véritable turn-over démocratique ; permettre à des bibliothécaires de terrain d'occuper la présidence sans mettre leur emploi en péril. Le mandat du président court d'une conférence annuelle (juin) à la suivante, ce qui fait que les dates du mandat sont toujours doubles (1995-96, par exemple). 
Les permanents (the staff) ne sont pas sur le terrain, ils ne travaillent pas en bibliothèque ni avec des bibliothèques. Nous, membres de l'association, nous sommes sur le terrain. ${ }^{283}$ " Autre exemple, en 1991, le président sortant, Richard Dougherty, est encore plus virulent - cette fois à l'égard du bureau : "L'ALA doit changer. Nous devons devenir une organisation moins complexe. Nous devons arriver à une meilleure définition du rôle des permanents, du bureau exécutif et du Conseil. Le Conseil est l'organe politique (the policy maker). Le bureau exécutif est l'outil de gestion. J'ai été témoin de tensions considérables. Le bureau exécutif n'est pas un bureau d'experts. Son rôle principal n'est pas d'être un groupe de visionnaires traçant l'avenir de l'association. ${ }^{284}$ » Tout au long des années 1990, les tensions restent vives - en 1992, la directrice exécutive est licenciée par le bureau en raison de " problèmes d'efficacité ». Une nouvelle directrice exécutive, nommée en 1994, claque la porte en 1996 en reprochant au Conseil de ne pas lui faire confiance ${ }^{285}$.

Ce sont donc, tour à tour, l'ensemble des membres, le bureau ou l'équipe salariée qui font figure d'accusés. La "réorganisation » de l'ALA semble être à l'ordre du jour depuis 50 ans et les rapports d'audit s'accumulent. Indépendamment de ces problèmes internes, l'audience de l'ALA continue à augmenter : 20000 en 1951 pour le $75^{\mathrm{e}}$ anniversaire de l'association, 35000 en 1969, 39000 en 1984, 58000 en 1999, plus de 66000 en 2008.

L'organisation entre composantes représentant les différents types de bibliothèque semble avoir permis un véritable équilibre. Équilibre de pouvoirs entre le global et le sectoriel : la Public Library Association (PLA) s'intéresse plutôt aux questions techniques et laisse le champ politique libre à la maison mère, l'ALA. Ainsi, c'est la PLA qui élabore les standards destinés aux Public Libraries et l'ALA qui prend en charge la promotion des bibliothèques ou les questions d'éthique. C'est pourquoi la PLA est réputée plus proche des bibliothécaires, plus concrète, plus utile - l'ALA plus lointaine, théorique, voire verbeuse ${ }^{286}$. Subtil partage des tâches qui semble bien fonctionner. Équilibre du poids entre les différentes divisions : en 2003, la PLA comptait 9344 membres, soit 14,6 \% des adhé- 
rents de l'ALA. C'était alors la troisième plus grosse section de l'ALA, après l'Association of College and Research Libraries (ACRL, association des bibliothèques d'étude et de recherche, 12261 membres) et l'American Association of School Librarians (AASL, association des bibliothécaires scolaires, 9669 membres).

\section{L'Association des bibliothécaires français (ABF)}

L'ABF est une association nationale organisée en sections (par types de bibliothèques) et en groupes régionaux. Elle est administrée par un Bureau national, qui émane du conseil d'administration (souvent appelé Conseil national), lui-même composé de représentants des sections et/ou des groupes (depuis 2007, le Conseil n'est plus constitué que de représentants des groupes régionaux). Son président est élu au sein du conseil d'administration pour un mandat de 3 ans renouvelable une fois. En 2009, elle comptait 3500 adhérents et 5 permanents.

L'ABF a été fondée (en 1906) sous l'aile tutélaire de la Bibliothèque nationale (BN) et cette origine pèse sur son développement. D’une part, l'association est, pendant longtemps, essentiellement parisienne, d'autre part, elle est pendant longtemps dirigée par des conservateurs de la $\mathrm{BN}^{287}$. En 1954, lorsque la vie de l'association reprend un cours normal après la guerre avec la reparution de son Bulletin d'informations, son président (Pierre Josserand) est conservateur à la BN ; son secrétaire général (Jacques Lethève) est bibliothécaire à la $\mathrm{BN}$; la présidente de la section de la lecture publique (Myriem Foncin) est conservateur à la BN. Des dix membres du bureau, dix travaillent dans des bibliothèques parisiennes. En 1956, ce sont naturellement les ors et les fastes de la Bibliothèque nationale qui accueillent le cinquantenaire de l'association. Le premier président provincial d'après-guerre sera Louis Desgraves, directeur de la BM de Bordeaux, élu en 1969. C'est en 1966 que, pour la première fois, l'assemblée générale de l'ABF se tient hors de Paris : à Neuilly-sur-Seine !

Fonctionnellement, cette double caractéristique, géographique et professionnelle, réduit son champ d'influence ; symboliquement, elle jette le doute sur sa représentativité. Ainsi, la création d'un syndicat des bibliothécaires municipaux, en 1932, est imputée par certains de ses fondateurs à « l'inertie de l'ABF ». Eugène Morel, dès 1908, se montrait sceptique sur 
l'influence que pourrait avoir l'association des bibliothécaires, créée deux ans plus tôt : "Pour la défense d'intérêts généraux. Ceux des bibliothèques et des bibliothécaires de France. Cela est beau, mais ce sont des intérêts divers. Celui du public n'est pas celui des bibliothécaires. S'alliant pour la défense de partis opposés, ils s'unirent contre eux-mêmes et marchèrent sur place. ${ }^{288} »$

L'homogénéité géographique et l'homogénéité professionnelle vont, ici, de pair. Les dirigeants de l'association sont des représentants des bibliothèques savantes. Depuis l'origine, la capacité de l'ABF à représenter toutes les bibliothèques et tous les bibliothécaires est fragile, voire contestée.

En 1959, c'est la première manifestation institutionnelle des bibliothécaires « modernistes », sous la houlette de Jean Hassenforder : la création d'une «section des petites et moyennes bibliothèques à rôle éducatif ». «Le mouvement se définit par une opposition à la prédominance des bibliothèques de conservation et à l'esprit qui les caractérise : accent sur une culture en relation avec le passé », se souvient Jean Hassenforder ${ }^{289}$, qui se rappelle aussi qu'alors (à la fin des années 1950) l'ABF « s'accommode de l'état des choses ", c'est-à-dire de la situation « consternante » des bibliothèques municipales, tandis que les bibliothécaires de lecture publique, eux, appellent « la mise en œuvre d'une action en vue du développement des bibliothèques publiques. » En 1964, cette section prend le nom de "section de la lecture publique ». En 1967, elle crée son propre bulletin (Lecture et bibliothèques, qui deviendra Médiathèques publiques et paraîtra jusqu'en 1988) et devient section « des bibliothèques publiques ».

Il faut attendre 1985 pour que soit élue présidente de l'association une représentante des bibliothèques publiques, et quelle représentante puisqu'il s'agit de la présidente de la section des bibliothèques publiques, Jacqueline Gascuel (qui plus est, une femme !), directrice de la bibliothèque publique de Massy ${ }^{290}$.

On peut voir dans cette montée en ligne des bibliothécaires de bibliothèques publiques à la fois l'affaiblissement symbolique des bibliothèques

288. Cité par Jacqueline Gascuel, Bulletin d'informations de l’ABF, n 162, 1994.

289. «Un mouvement pour le développement des bibliothèques publiques : les origines de la Section des bibliothèques publiques de l'Association des bibliothécaires français », Médiathèques publiques, $n^{\circ}$ 71-72, 1988.

290. Jacqueline Gascuel analyse ainsi son élection de 1985 : « La pression du nombre a fait que la culture élitiste ne pouvait plus être la seule à représenter l'ABF. » (Jacqueline Gascuel, « Mon slogan : résister », BIBLIOthèques, $\mathrm{n}^{\circ} 2$, avril 2002). 
savantes dans les années 1980 et 1990, et la reconnaissance du fait que l'ABF ne représente plus que les bibliothèques publiques, les autres types de bibliothèque ayant créé leurs propres instances de représentation (l'ADBU pour les bibliothèques universitaires en 1970, l'ADBDP pour les bibliothèques départementales en 1987, l'ADBGV pour les grosses bibliothèques municipales en 2002). En 1981, la section des bibliothèques publiques représente $60 \%$ des adhérents, $70 \%$ en 1986 (Bulletin d'informations de l'ABF, $\mathrm{n}^{\circ}$ 132, 1986), $80 \%$ en 1998 (Bulletin d'informations de l'ABF, $\mathrm{n}^{\circ} 184$ 185, 1999). Pour freiner ce déséquilibre croissant, il est procédé en 1993 à la fusion des sections BN, BS et BU dans une grande " section étude et recherche ". La dissolution de toutes les sections est votée au congrès de 2004, prenant acte de l'obsolescence de ce découpage. L'ABF n'en demeure pas moins marquée par cette sur-représentation des bibliothécaires travaillant dans les bibliothèques publiques.

\section{Activités et rôle}

L'histoire, la taille, la représentativité des deux associations (1 bibliothécaire américain sur 2 est adhérent de l'ALA, 1 bibliothécaire français sur 10 de l'ABF) génèrent inévitablement une grande différence dans le positionnement et le rôle que joue chacune des associations.

Dans la logique française de co-gestion de segments administratifs, l'ABF a toujours été proche du pouvoir : plutôt un pouvoir bis qu'un contrepouvoir, elle cherche davantage à influencer les décideurs qu'à lutter contre leurs décisions. Jusque dans les années 1970, la modestie des effectifs rend possible un travail d'influence basé sur la familiarité et la complicité : on constate des allées-et-venues entre l'administration centrale (ou l'Inspection générale des bibliothèques) et les ténors de la profession, qu'ils soient responsables associatifs ou leaders d'opinion. Rappelons que la jeune Direction chargée des bibliothèques (la DBLP) est créée en 1944 avec le concours actif de Pierre Lelièvre, alors président de l'ABF, et de Paul Poindron, son secrétaire général. Henri Vendel, nommé inspecteur général en 1945, célèbre promoteur du bibliobus de la Marne, a été président de l'ABF de 1938 à 1940. En 1970, Louis Desgraves (élu président de l'ABF en 1969) est nommé inspecteur général des bibliothèques - et démissionne de la présidence. En 1990, Françoise Danset (élue présidente l'année précédente) est recrutée par la Direction du livre pour s'occuper du secteur du « développement de la lecture ». 
Qu'il y ait communauté de vues entre l'ABF et l'administration centrale est logique, puisque la DBLP a été créée avec et par des responsables de l'association et que l'ABF est constamment associée aux travaux de la Direction (DBLP puis DL-L), par exemple au sein du comité interministériel (1966-1967), du groupe de travail sur les bibliothèques municipales (1973-1974) ou du groupe de travail « décentralisation et bibliothèques publiques » (1984). Cette proximité durera un demi-siècle. C’est la même culture professionnelle, ce sont les mêmes objectifs qui animent les uns et les autres.

On peut parler d'institutionnalisation de l'ABF. En 1969, l'ABF est reconnue d'utilité publique. La subvention de l'État (principalement la Direction du livre, souvent accompagnée d'une modeste aide du ministère des Affaires étrangères) augmente progressivement, au fur et à mesure que l'ABF grandit et que son budget augmente. Mais la subvention augmente plus vite que le budget et représente ainsi une part grandissante des recettes : encore marginale dans les années 1970 (7\% du budget en 1974), elle augmente progressivement jusqu'à un pic de $36 \%$ en $1985^{291}$, puis diminue : $28 \%$ du budget en 1987, $19 \%$ en $2001^{292}$.

L'ABF cherche à influencer, non à s'opposer. Échanger, discuter : c'est le ministère de la parole qu'elle exerce. "L'avenir de nos établissements continue à nous préoccuper - et nous avons eu l'occasion de le dire à plusieurs reprises, soit de vive voix à nos Directeurs, soit à travers nos motions et communiqués de presse, soit en écrivant aux Ministres de tutelle et aux candidats à la Présidence de la République », explique Jacqueline Gascuel dans son rapport moral en $1988{ }^{293}$. Encore l'année 1988 fut-elle une année électorale, avec des candidats plus attentifs. Mais 1986 fut encore moins encourageante : « Le retard des bibliothèques françaises est un mal qui a une longue histoire et que certains préfèrent ignorer : le rapport préparé par nos deux directeurs ${ }^{294}$ n'a jamais été publié et il n'a point été répondu à nos lettres demandant cette publication ! [...] Nous avons renouvelé nos demandes d'audience lors de la nomination du nouveau gouvernement. Votre bureau, ceux des sections sont vigilants... mais il est difficile d'être reçu et plus encore d'être entendu. Probablement nous faudrait-il un appui plus solide des lecteurs, des écrivains, des cher- 
cheurs, des universitaires, de la presse... Nous devrions l'obtenir. ${ }^{295}$ » Analyse lucide : si l'ABF est peu audible, c'est que les bibliothèques n'ont pas réussi à mobiliser derrière elles la société civile.

Lors du congrès de 1988, un atelier de réflexion est organisé sur l'image de l'ABF. Le constat est sévère : " L'ABF est trop souvent oubliée comme interlocuteur à l'étranger ; par exemple, dans les instances internationales, on constate que nos collègues étrangers sont représentés par leurs associations professionnelles. La France l'est par ses deux directions (la DLL et la DBMIST), non par l'ABF. L'ABF doit s'affirmer pour être réellement une force de pression, en demandant, en exigeant d'être associée à un certain nombre de réflexions et de décisions. ${ }^{296}$ " Être associée aux réflexions et aux décisions : c'est dans cette ligne constructive, coopérative, que l'ABF se place délibérément. On est bien loin de la force tranquille de l'ALA.

On a déjà vu que, par le biais de la Public Library Association, l'ALA a élaboré les standards sur lesquels s'est construit le développement des années 1950 et 1960 - il est à noter que cette fonction normative appartient, en France, à la structure étatique. Elle les met régulièrement à jour pendant les décennies suivantes. Elle joue un autre rôle para-public, qui est celui de l'accréditation des écoles de bibliothécaires : un processus complexe et régulier permet à l'ALA de valider le niveau de formation des écoles de bibliothécaires aux États-Unis, mais aussi au Canada. L'ALA cogère, avec la Library of Congress et la British Library, la révision et la mise à jour des Anglo-American Cataloguing Rules. Elle surveille le recrutement des bibliothécaires, leur niveau de qualification ou de salaire, la diversité ethnique, la place des femmes, etc. Elle publie, directement ou par le biais de ses divisions ou associations locales, des dizaines de bulletins, revues ou magazines. Elle édite un nombre important d'ouvrages - plus de 300 titres au catalogue en 2005. Elle mène des relations internationales actives, en particulier au sein de l'International Federation of Library Associations (IFLA).

Mais le rôle majeur que joue l'ALA, et qui la distingue le plus de l'ABF, est son rôle de porte-parole. 


\section{ADVOCACY}

\section{Porter la parole}

Communiquer, plaider, défendre, expliquer, convaincre, argumenter : le rôle de porte-parole de l'ALA a des aspects, des vecteurs et des expressions multiples.

D'une certaine façon, on peut considérer que le président de l'ALA en est d'abord son porte-parole ${ }^{297}$. Que les thèmes choisis par les présidents pour leur présidence sont des slogans («Égalité d'accès », " Diversité », "Les enfants qui lisent réussissent », "Construire la communauté »). Que les conférences annuelles ou les réunions de Midwinter sont des tribunes. Que le vrai cœur de l'ALA bat à Washington, au Washington Office chargé du lobbying auprès des parlementaires et des autorités fédérales, et non à Chicago ${ }^{298}$. Que les publications de l'ALA sont aussi des relations publiques - comme le suggèrent, par exemple, les posters où Batman, Mel Gibson, Bill Gates ou la soprano Renee Fleming invitent à la lecture. Qu'une partie notable du site de l'ALA sert à fournir des argumentaires, comme « Top 10 Reasons to Support Libraries " (10 bonnes raisons pour soutenir votre bibliothèque), "The Top 10 Reasons To Be A Librarian » (10 bonnes raisons pour devenir bibliothécaire) ou " 10 Reasons Why the Internet Is No Substitute For a Library " (10 raisons pour lesquelles Internet ne remplace pas les bibliothèques). Que les campagnes récentes @your library (l'affirmation que les bibliothèques restent indispensables, malgré Internet), Every Child Ready to Read (les bébés-lecteurs), The Smartest Card (campagne d'inscription dans les Public Libraries) absorbent délibérément une énergie considérable. Que les nombreux événements qui marquent l'année sont autant d'occasions de faire parler des bibliothèques et de la lecture - citons la National Library Week (la semaine nationale des bibliothèques, co-organisée avec les éditeurs ${ }^{299}$ ), la Teen Read Week (la semaine

297. Le président reçoit un media-training, il répond à de nombreuses interviews (150 pour Betty Turock, présidente en 1995-1996), on le voit sur les plateaux de la télévision, en 2003 Carla Hayden, alors présidente, est désignée comme l'une des dix femmes de l'année par le magazine Ms.

298. Par deux fois, en 1957 et en 1967, un vote des adhérents a interdit le déménagement du siège de l'ALA à Washington, pourtant décidé par le Conseil.

299. Popularisée dans le monde entier par Charles Schulz, l'auteur des Peanuts, qui fait dire à Charlie Brown : " J'emprunte toujours un livre à la bibliothèque pendant la National Library Week parce que cela soutient le moral des bibliothécaires. Les bibliothécaires aiment se sentir utiles. » 
de la lecture des jeunes), la Banned Book Week (la semaine des livres censurés, depuis 1982), le Legislative Day (le jour des parlementaires), le Library Card Sign-up Month (le mois des adhésions).

Communiquer, promouvoir, défendre. Un terme anglais, à vrai dire intraduisible, résume cette activité primordiale : advocacy. Cette activité n'a pas d'équivalent en France.

\section{Professionnaliser}

Un des objectifs qui rapprochent, par contre, l'ALA et l'ABF est le souci de promouvoir la profession de bibliothécaire, d'en améliorer les qualifications, les niveaux de recrutement, l'image et la réputation.

La formation des bibliothécaires est, depuis longtemps, une préoccupation de l'ALA. La première école de bibliothécaires est créée en 1887 par Melvil Dewey. En 1924, l'ALA crée son Board of Education for Librarianship, qui élabore des standards destinés à évaluer et accréditer les écoles. La première vague d'accréditation a lieu en 1925-1926. Les années 1940 à 1960 sont considérées comme un âge d'or où les bibliothèques se développent, les emplois se créent, les écoles de bibliothécaires fleurissent et prospèrent. Retournement de situation dans les années 1970 : sous le coup de la crise économique (les études de bibliothécaire sont relativement chères par rapport au salaire attendu) et du début de la révolution informatique, le nombre des élèves dans les écoles de bibliothéconomie décroît nettement, de 5137 étudiants en 1976 à 3160 en $1986^{300}$. Pis, les écoles commencent à fermer car les universités, elles aussi, traversent une passe difficile : après celle de l'université d'Oregon, en 1978, une quinzaine d'autres, dont les prestigieuses écoles de l'université de Chicago et de Columbia University ferment dans les années 1980. Alors même que l'école de bibliothécaires de Columbia (Columbia University's School of Library Service) venait de célébrer en grande pompe son centenaire, sa fermeture fait l'effet d'une bombe.

Dans ce contexte difficile, l'ALA semble plutôt démunie. Peu à peu, les interrogations se focalisent sur la conjonction entre la formation des bibliothécaires et les sciences de l'information : Library and Information Science (LIS) est le nouveau nom des écoles, adopté presque unanimement dans les années 1980 avant que le L de Library ne tende à carrément disparaître. Terry Weech date précisément cette évolution : sur la 
soixantaine d'écoles accréditées par l'ALA, 1 faisait figurer « sciences de l'information » dans son nom en 1965, 9 en 1975, 42 en 1985 et presque toutes en $1995{ }^{301}$. Il explicite par la même occasion une question que beaucoup de bibliothécaires se posent : dès lors, « comment les écoles qui forment les bibliothécaires se différencieront de celles qui forment les informaticiens ou les ingénieurs de secteurs voisins "? Enseignant à Montréal, Réjean Savard, quelques années plus tard, pose autrement la même question : "Malgré l'importance que l'on peut accorder aux nouvelles technologies, celles-ci ne sont que des outils [...]. Si l'on en croit les programmes de formation continue des bibliothécaires où les nouvelles technologies écrasent de tout leur poids les autres sujets de formation (du moins en Amérique du Nord), on peut se demander si cette profession a un intérêt pour autre chose que les applications informatiques à la bibliothéconomie. ${ }^{302}$ »

Cette « insatisfaction croissante » confine à la crise d'identité. L'ALA décide alors de tenir un congrès spécifique sur la formation professionnelle. Il a lieu à Washington en 1999 et se conclut par la création d'un groupe de travail chargé de définir les valeurs centrales (Core Values Task Force), dont la présidence est confiée à Donald Sager. En 2000, au congrès annuel, les travaux du groupe de travail sont présentés au Conseil de l'ALA sous forme de projet de résolution. Ce document de 15 lignes, déjà amendé après avoir été mis en discussion auprès des divisions et des associations régionales de l'ALA, provoque des remous dans le Conseil. S'il est approuvé par quelques conseillers, deux objections majeures lui sont faites. L'une, de prétendre établir la liste des valeurs qui guident l'ALA et la profession de bibliothécaire tout entière alors que l'ALA possède déjà un nombre de documents impressionnant en ce domaine (Policy Manual, Library Bill of Rights, Freedom of Read Statement, Code of Ethics, etc.). L'autre, de ne pas citer explicitement la liberté d'information (Intellectual Freedom) alors qu'elle est la valeur centrale, nodale, de l'ALA - le document cite seulement « la liberté pour tous de former, soutenir et exprimer ses propres convictions ${ }^{303}$ ". Après une longue discussion, le Conseil décide de renvoyer le document au bureau de l'association. Classique

301. Terry L. Weech. « L'évolution des métiers des bibliothèques aux États-Unis ». Bulletin des bibliothèques de France, t. 40, $\mathrm{n}^{\circ}$ 6, 1995.

302. Réjean Savard. « La formation des bibliothécaires en Amérique du Nord : modernité et valeurs professionnelles ». Bulletin des bibliothèques de France, t. $45, \mathrm{n}^{\circ} 1,2000$. Il signale qu'à l'EBSI (Montréal), « des cours sur l'histoire du livre et des bibliothèques, l'édition, la littérature de jeunesse, la gestion du personnel en bibliothèque, etc., ont été éliminés ».

303. ALA Council Minutes. 2000 Annual Conference, Chicago. 
manœuvre d'enterrement : le bureau confie l'élaboration d'un nouveau texte à un deuxième groupe de travail qui, après quelques atermoiements, remet en 2004 un bref document que le Conseil adopte sans même le discuter. Il confie au Policy Monitoring Committee le soin de l'intégrer dans le Policy Manual ${ }^{304}$.

Le lien entre les valeurs professionnelles et la formation des futurs professionnels a été perdu de vue. Et Donald Sager pouvait tristement commenter : "Sans un ensemble de valeurs communes, nous ne pouvons pas être considérés comme une profession. ${ }^{305}$ »

La formation est une des activités fondatrices de l'ABF. Très vite, elle met en place des formations, plaide pour la création de diplômes reconnus, s'investit dans l'élaboration de manuels.

L'idée de formation mutuelle guide l'ABF : les futurs professionnels sont formés, doivent être formés par leurs prédécesseurs. Dès avantguerre, des pionniers comme Ernest Coyecque ou Eugène Morel donnent des cours à l’École américaine. En 1938, l'ABF met en place une formation professionnelle pour les futurs responsables de petites bibliothèques. Après la guerre, la même logique prévaut : les cours de préparation au DSB (diplôme supérieur de bibliothèque) ou au CAFB (Certificat d'aptitude aux fonctions de bibliothécaire) sont assurés presque intégralement par des praticiens. La création de bibliothèques d'application (Clamart en 1963, Massy en 1971) participe de cette idée de tutorat, l'ancien accompagnant le nouveau à son entrée dans le métier. Il n'est pas indifférent que Noë Richter, au plus fort de la lutte pour les bibliothèques de secteur, en 1972, ait accepté la direction de l'ENSB. Aujourd'hui, la plupart des groupes régionaux de l'ABF continuent à assumer une formation d'auxiliaire de bibliothèque.

Cette formation doit nécessairement déboucher sur la professionnalisation. Pendant longtemps, en France, le métier de bibliothécaire n'est pas considéré comme un métier. Il est vrai que le premier diplôme destiné à la formation du personnel territorial, le CAFB, n'est créé qu'en 1951 et sa préparation assurée seulement à partir de 1960. Il connaît un lent développement pendant les années 1950 (en 1961 seulement 29 lauréats), puis un essor rapide dans les années 1960, la promotion de 1971 atteignant 197 diplômés. Le DSB n’est créé qu'en 1950, l’ENSB qu'en 1963 (elle 
se muera en enssib en 1992). L'amélioration de la qualification est statutairement encouragée, par l'arrêté du 24 janvier 1966, qui fait du CAFB (ou du DSB) un diplôme exigé pour le recrutement sur titre des bibliothécaires ou sous-bibliothécaires dans les bibliothèques municipales. Cet arrêté sonne le glas des sinécures occupées par la femme du secrétaire général, l'archiviste départemental ou le professeur en retraite.

Pour accompagner ce mouvement de professionnalisation, l'ABF publie cours et manuels. Son œuvre la plus connue est Le métier de bibliothécaire, dont l'association est l'auteur collectif ; la revendication professionnalisante est bien soulignée par le titre. Héritier des manuels du début du $\mathrm{xx}^{\mathrm{e}}$ siècle, Le métier de bibliothécaire est d'abord un recueil de cours ${ }^{306}$ qui a évolué progressivement vers un ouvrage de synthèse et de réflexion. Il est considéré comme « le manuel de base », le bagage minimum des professionnels de la lecture publique et de ceux qui aspirent à le devenir - il est ainsi utile à la fois dans l'exercice du métier et dans la préparation aux concours de recrutement. C'est l'ouvrage sans doute le plus connu et le plus lu de la littérature professionnelle (bibliothécaire) française. Il a été publié onze fois entre 1966 et 2003, sa pagination n'a cessé de croître (de 167 pages pour la $1^{\text {re }}$ édition à 454 pages pour la $11^{\mathrm{e}}$, signe de la complexité croissante du métier et de l'extension de son champ d'activité). L'ampleur qu'a pris cet ouvrage l'a détourné de sa mission originelle et a amené l'ABF à recréer un manuel de base, le Mémento du bibliothécaire, dont la première édition date de 2006.

\section{DEUX COMBATS EXEMPLAIRES}

Pour clore ce chapitre associatif, je vais illustrer les différences culturelles entre les deux associations par l'évocation de deux combats exemplaires, l'un, de ce côté de l'Atlantique, le combat pour une loi sur les bibliothèques ; l'autre, de l'autre côté, le combat pour la liberté d'information.

\section{La demande $d$ 'une loi sur les bibliothèques}

La constance avec laquelle l'ABF appelle de ses vœux une loi sur les bibliothèques n'a d'égal que la persistance avec laquelle ce projet est repoussé : 
" Loi. On dit “la loi” sans préciser laquelle ni rappeler qu'elle n'existe pas. C'est la loi sur les bibliothèques que nous appelons de nos vœux depuis plus d'un demi-siècle. Elle nous permet, dans les assemblées générales ou face à un représentant des "tutelles", de prendre soudain un air grave. Tel l'horizon, elle recule à mesure que nous avançons. N'est-ce pas très bien comme ça ? ${ }^{307}$ » La description amusée du phénomène, sous la plume de Dominique Lahary, montre bien le caractère à la fois rituel et répétitif de la « demande de loi ». De quelle loi s'agit-il ?

À la Libération, l'ABF et l'administration centrale (Direction des bibliothèques et de la lecture publique, puis Direction du livre, puis Direction du livre et de la lecture) partagent la même analyse : la loi doit obliger les communes à créer et financer des bibliothèques pour que tous les habitants du pays bénéficient du même service public (préoccupation partagée aux États-Unis, où le nombre d'habitants non desservis est régulièrement mis en avant dans les années 1950 et 1960 pour appeler à de nouveaux efforts). En 1944, le projet d'organisation des bibliothèques, dit « rapport Lelièvre », prévoit un " texte impératif », c'est-à-dire une loi, pour obliger les communes à financer une bibliothèque : « Les villes comprennent très inégalement leurs devoirs. Il serait souhaitable qu'elles fussent contraintes par un texte impératif de consacrer une somme proportionnelle au nombre d'habitants agglomérés à l'entretien de la bibliothèque municipale (2,50 F, valeur 1944, par tête d'habitant) ». De même, le rédacteur du rapport de $1948{ }^{308}$ considère que les problèmes financiers des bibliothèques municipales resteront « difficilement surmontables, tant qu'une loi sur les bibliothèques n'aurait pas rendu obligatoire le vote par les villes de crédits proportionnels à leurs ressources et à leur population. »

En 1968, les Assises des bibliothèques, organisées par l'ABF, reprennent à leur compte la demande d'une loi : « La réalisation d'une enquête nationale sur la situation des bibliothèques publiques en France et sur les résultats qu'elles obtiennent est considérée unanimement comme indispensable et urgente. La publication du rapport d'enquête servirait de base à l'évaluation des besoins et de l'effort nécessaire pour que le retard déjà considérable de la France dans le domaine des bibliothèques publiques ne s'accroisse pas. Il devrait en résulter un texte législatif définissant les devoirs respectifs des collectivités nationales et locales et rendant effectif

307. Dominique Lahary, « L'ABF de A à Z », BIBLIOthèques, $\mathrm{n}^{\circ} 2$, avril 2002. La première trace d'une demande de loi ne remonte pas à un demi-siècle mais à 1910.

308. La lecture publique en France. La Documentation française (Notes documentaires et études, $\mathrm{n}^{\circ}$ 918). 
le droit de tous les Français à la culture par le livre. ${ }^{309}$ » En 1972, l'Assemblée générale de l'ABF renouvelle ce souhait par une motion ${ }^{310}$. Cette rhétorique ancienne et partagée finit par porter ses fruits : le Conseil des ministres du 8 janvier 1979 reconnaît le développement des bibliothèques municipales comme " un objectif prioritaire de l'action culturelle ", et la Direction du livre est chargée d'élaborer un projet de loi pour « définir les missions et les obligations respectives de l'État et des collectivités locales en matière de lecture publique ». Le projet de texte est validé au Conseil des ministres du 20 juillet 1979.

La section des bibliothèques publiques, pour sa propre part, soutient ce projet au congrès de La Rochelle, en juin 1979 : « La section des bibliothèques publiques de l'ABF se prononce pour l'élaboration et l'adoption d'une loi sur les bibliothèques. Cette loi devrait stipuler que la création de conditions d'accès égales, libres et gratuites pour tous les habitants du pays à la totalité des fonds documentaires sans discrimination entre les divers types de documents est une obligation commune de l'État et des collectivités locales. ${ }^{311}$ "

Ce projet de projet de loi, né trop tard, avortera silencieusement au début des années 1980 - la logique de décentralisation l'emportant désormais sur la logique de l'aménagement du territoire mené sous la houlette experte de l'État.

Mais l'idée qu'une loi est nécessaire mettra plus de temps à mourir que le projet de loi lui-même. En 1981 encore, le rapport Vandevoorde ${ }^{312}$ préconise l'adoption de deux lois : une loi-programme quinquennale permettant de financer la construction de $300000 \mathrm{~m}^{2}$ de locaux et une « loi sur les bibliothèques " qui " serait, mutatis mutandis, une manière de traiter la lecture publique et ses agents de la même manière que l'éducation. Deux possibilités : soit une loi d'orientation de l'action communale, énumérant les principes et les conditions de développement, soit une loi d'obligation imposant aux communes le respect de certaines exigences : l'article central d'une telle loi ne pourrait être alors que l'obligation faite aux communes de plus de 10000 habitants de mettre à la disposition des

309. «Les Assises nationales des bibliothèques, 8-10-juillet 1968 ». Bulletin d'informations de l'ABF, $\mathrm{n}^{\circ} 61,1968$.

310. Bulletin d'informations de l'ABF, $\mathrm{n}^{\circ} 76,1972$.

311. Bulletin d'informations de l'ABF, $\mathrm{n}^{\circ} 105,1979$.

312. Achevé en juillet 1981, publié en mars 1982 (Les bibliothèques en France, Dalloz), le rapport Vandevoorde a été demandé par Raymond Barre à Pierre Vandevoorde, mais publié sous le gouvernement Mauroy, Jean Gattégno étant directeur du livre. 
habitants une bibliothèque municipale obéissant à certaines définitions, plus ou moins précises, plus ou moins contraignantes. »

Le discours est donc, pendant presque quarante ans, intangible : les villes ne s'intéressant pas, ne s'investissant pas dans/pour leurs bibliothèques, l'État doit les y contraindre par la loi. Étrange entêtement. Doublement étrange : d'une part, l'inefficacité de la démarche ne semble pas gêner les partisans de la loi, qui persévèrent à travers les décennies ${ }^{313}$. D'autre part, le développement des bibliothèques municipales à partir des années 1970, qui s'accélère dans la décennie 1980, en l'absence de toute loi d'obligation, pourrait éclairer sur l'inanité de la démarche. Non seulement, la demande de loi n'aboutit pas, mais encore les bibliothèques municipales se développent sans loi. La loi est à la fois inaccessible et inutile - d'où le « N'est-ce pas très bien comme ça ? » de Dominique Lahary.

Bien plus : l'État n'est plus aujourd'hui en position d'imposer des contraintes normatives aux collectivités locales (excepté en matière régalienne, comme pour l'état-civil ou l'ordre public). Depuis les lois Defferre (2 mars 1982 et 22 juillet 1983, pour celles qui intéressent les bibliothèques), la logique des lois de décentralisation est bien, au contraire, d'alléger l'encadrement des collectivités territoriales et d'accroître leur autonomie. La lassitude aurait peut-être gagné l'ABF si un nouvel argument n'était apparu en 1995-1996 avec l'affaire des bibliothèques gérées par le Front national. Une nouvelle fois, l'ABF réagit en demandant à l'État de protéger les bibliothèques de tels agissements - bien sûr, en faisant adopter une loi. La motion adoptée en 1996 est très générale, mais celle de 1997 fait explicitement allusion aux problèmes de censure à Marignane, Orange

313. Au congrès de Strasbourg, en 1980, comme au congrès de Saint-Brieuc, en 1983, une motion est adoptée par l'ABF pour demander que le projet de loi de 1979 aille à son terme (Bulletin d'informations de l'ABF, $\mathrm{n}^{\circ} 109,1980$, et $\left.\mathrm{n}^{\circ} 119,1983\right)$. En 1992, un dossier «Quelle loi pour les bibliothèques en France ?» est publié dans le Bulletin d'informations de l'ABF ( $\left.\mathrm{n}^{\circ} 154,1992\right)$. En 1996, les travaux de la « Commission Loi » de l'ABF avancent cet attendu : «Si le législateur décide que la constitution doit être appliquée, qui affirme le droit pour tous les citoyens à l'information, l'instruction et la culture, il est nécessaire qu'il organise dans la loi la mise en œuvre réelle par la collectivité de ces droits. » (Claudine Belayche, « Une loi sur les bibliothèques », Bulletin d'informations de l'ABF, $\left.\mathrm{n}^{\circ} 172,1996\right)$ En 2000, Claudine Belayche regrette que « cette loi semble reportée sine die » ( $\left.{ }^{\circ} 187,2000\right)$. En 2001, l’ABF réaffirme, dans son assemblée générale, son « exigence de mise en chantier rapide d'une concertation sur un projet de loi sur les bibliothèques. » $\left(\mathrm{n}^{\circ} 192\right.$, 2001). Le rapport d'orientation pour 2002-2003 écrit : les bibliothèques « ne peuvent dépendre des caprices politiques, [elles] doivent devenir des institutions réellement démocratiques auxquelles les usagers, les élus, les professionnels doivent pouvoir contribuer et en orienter le fonctionnement à partir de missions définies dans une loi garantissant le droit de chaque citoyen. » (Note d'informations, $\mathrm{n}^{\circ}$ 99, août 2002). L'assemblée générale du congrès de 2009 adopte une " Plate-forme préparatoire à une loi sur les bibliothèques ". 
et Vitrolles, puisqu'il est précisé dans la motion que la loi doit avoir pour objet « de garantir la responsabilité des bibliothécaires notamment dans la constitution et l'organisation des collections et dans la gestion des établissements ; et de rappeler que les bibliothèques sont un des moyens d'exercice de la démocratie ${ }^{314}$ » tandis qu'une autre motion, votée le même jour, apporte le soutien de l'ABF « aux professionnels qui œuvrent dans des villes où les tenants d'une idéologie totalitaire tentent de transformer des bibliothèques publiques, par fonction outils d'exercice de la démocratie, en supports de propagande politique ».

L’ABF demande à l'État d'assurer le développement des bibliothèques, leur organisation et leur défense vis-à-vis des élus locaux. La demande d’État n'a, on le sait, pas de sens aux États-Unis.

\section{La liberté d'information}

La défense de la liberté d'information est une préoccupation commune aux bibliothécaires des deux pays. L'implication qu'elle suscite, les moyens employés, l'éclat donné à ce combat diffèrent cependant du tout au tout d'un pays à l'autre.

Jusqu'en 1995, les bibliothécaires français n'accordaient pas à la censure une attention particulière. Quelques attaques se faisaient jour, chroniquement $(1983,1987)$, surtout contre les collections de livres pour enfants, accusées évidemment de corrompre la jeunesse. Un ouvrage historique sur la censure ${ }^{315}$, publié en 1989, n'avait suscité qu'un intérêt poli et légèrement incrédule - pas nous, pas ça. Mais, globalement, le sujet n’intéressait pas : nul comité pour la liberté d’information, nul texte de référence, nulle expertise ou mobilisation en ce domaine. Dans les bibliothèques françaises, la censure n'existait pas - ou, plus exactement, le concept de censure n'existait pas.

L'épisode qui peine à trouver un nom (« les agissements » du Front national est une formule courante) est une conséquence des élections municipales de 1995 : les électeurs de trois villes élisent démocratiquement des membres du Front national pour diriger leur municipalité (Marignane, Orange, Toulon ; s'y ajoutera Vitrolles dans une élection partielle en 1997). Ce n'est qu'à l'été 1996 que, après quelques mois d'exer-

314. Bulletin d'informations de l'ABF, $\mathrm{n}^{\circ} 176,1997$.

315. Marie Kuhlmann, Nelly Kuntzmann, Hélène Bellour. Censure et bibliothèques au XXe siècle. Éditions du Cercle de la Librairie, 1989. 
cice abusif (mais non illégal) du pouvoir que les élus ont sur les bibliothèques, l'affaire éclate au grand jour - grâce à la diffusion du rapport que l'Inspection générale des bibliothèques avait remis au ministre. La presse s'empare alors de cette actualité (Libération 11 juillet 1996, Le Monde 12 juillet 1996, New York Times 20 août 1996).

Jo Kibbee, bibliothécaire à l'université d'Illinois, raconte ainsi l'épisode d'Orange : "L'enquête diligentée par le ministère fut confiée à Denis Pallier, alors doyen de l'Inspection générale des bibliothèques. Selon le rapport qu'il remit par la suite au ministre, les collections de la BM d'Orange avaient été "enrichies" de nombreux ouvrages publiés à compte d'auteur par des sympathisants du FN, tel Max Rodolphe François, ce contre l'avis de la directrice de la bibliothèque qui les jugeait partisans et d'une piètre qualité littéraire. Le rapport décrit également la révision sans nuances des listes soumises par le service des acquisitions, le bureau du maire n'hésitant pas à éliminer des titres en se fondant sur des critères conformes au programme politique du FN. Les annotations portées dans les marges précisaient les raisons du rejet: multiculturalisme (pour un recueil de contes et légendes du monde entier, par exemple); atteinte aux "bonnes mours" (pour un roman où il était question d'homosexualité) ; ouvrages signés d'auteurs connus pour leurs positions critiques à l'égard du FN (l'historien Jean Lacouture), et autres motifs. »

Jo Kibbee, «Aux armes citoyens ! Les bibliothèques publiques françaises face à l'extrême droite », Bulletin des bibliothèques de France, t. 49, nº 6, 2004.

La réponse de l'ABF (au niveau national) fut longue à venir, peu claire et peu active. Ce n'est qu'après la presse générale (Libération, Le Monde) que l'ABF aborde publiquement le sujet. Dans un éditorial du Bulletin d'informations, la présidente évoque en termes pudiques «l'affaire » qui fait la une de la presse nationale : "Cet été a été aussi celui d’inquiétudes fortes, que nous connaissions déjà et dont la presse nationale s'est fait largement l'écho, concernant les pressions auxquelles certains de nos collègues sont soumis dans l'exercice de leur métier, et particulièrement dans cette activité fondamentale qu'est la mise en œuvre d'une politique d'acquisitions de livres. » $\left(\mathrm{n}^{\circ} 172,1996\right)$ Les mots " censure », " Front national », " Orange » ou « Marignane » sont soigneusement évités. Parallèlement, à partir de l'automne 1996, l'ABF fait signer une pétition 
où ces mots ne figurent pas davantage (sauf « Orange ") ${ }^{316}$. En octobre 1997, l'ABF publie dans la presse nationale une "Lettre ouverte aux élus de la République et aux élus locaux » avec le même implicite.

La section des bibliothèques publiques est plus (ré)active que l'association dans son ensemble. C'est la section qui organise une journée d'étude en mars 1997. Son rapport d'activités, au congrès 1997, parle explicitement des « atteintes au pluralisme par le Front national » et se veut «l'observatoire, l'analyste des menées du Front national » ( $\left.n^{\circ} 176,1997\right)$. Mais enfin, l'ambition d'être " l'observatoire, l'analyste », si elle est louable, est aussi un renoncement à toute action - dans le même numéro, l'éditorial de la présidente continue à évoquer mystérieusement « des attaques frontales menées dans certaines communes contre une conception large de l'information et de la documentation ».

Au niveau national, on le voit, l'embarras semble grand. Ce qui explique sans doute la ligne corporatiste choisie par l'ABF : dans cette affaire, ditelle, il faut défendre la responsabilité professionnelle des bibliothécaires sur les acquisitions. Une grave et délibérée activité de censure sera désormais traitée par l'ABF sous l'enseigne « les acquisitions dans les bibliothèques publiques ». Une commission portant ce nom est créée en 1997, au congrès de Bordeaux.

Comment expliquer les réticences de l'ABF, au niveau national, à s'impliquer explicitement et vigoureusement contre la censure exercée par des élus du Font national ${ }^{317}$ ? On peut invoquer l'absence d'expérience (et donc l'absence d'outils). Des responsabilités mal définies entre l'échelon national et l'échelon régional (très actif, lui). Et, surtout, les divisions de l'association. Ces réactions, ces débats, qui peuvent sembler bien en deçà de l'enjeu, apparaissent au contraire à certains comme abusifs, excessifs, comme une «dérive » politique : le groupe Paris de l'ABF écrit le 24 sep-

316. Le texte commence ainsi : « Les bibliothécaires, membres de l'ABF, sont inquiets après les informations révélées sur le fonctionnement de la Bibliothèque municipale d'Orange, et d'autres établissements de lecture publique [...] ».

317. Jo Kibbee doute de la portée des réactions de l'ABF : «Si utiles qu'elles soient, ces démarches ne sont sans doute pas en elles-mêmes suffisamment dissuasives pour décourager le FN, ou d'autres groupes partisans, de transformer la bibliothèque en plate-forme idéologique et de s'en prendre aux bibliothécaires. Raison pour laquelle d'aucuns ont lancé l'idée de promulguer une loi qui protégerait concrètement l'autonomie des bibliothèques et des bibliothécaires. " (Jo Kibbee, " Aux armes citoyens ! », op. cit.) Une intervenante, lors de la journée d'étude de 1997 « Les acquisitions dans les bibliothèques publiques », exprime sa frustration : « Les débats, les interrogations, les commissions sont utiles, mais il y a une situation d'urgence, des situations de censure dans les bibliothèques et des journaux qui diffament et propagent la haine. Il faut agir. S'il faut faire des procès, faisons des procès, allons dans telle ou telle bibliothèque, s'il faut faire grève, faisons grève. » (Bulletin d'informations de l'ABF, $\mathrm{n}^{\circ} 175,1997$ ) 
tembre 1997 une « Lettre ouverte au Bureau national de l'ABF » pour se plaindre du dernier congrès : " Il est souhaitable qu'à l'avenir les présidents de séance aient une meilleure maîtrise des débats, qu'ils assurent un équilibre entre les divers courants et tendances, qu'ils privilégient l'expression des problèmes professionnels et techniques plutôt que celle d'opinions à coloration corporatiste ou syndicale, voire politique. Il existe d'autres lieux pour débattre de ces problèmes en dehors de l'Association. ${ }^{318}$ "

En somme, en France, des bibliothécaires doutent que la censure soit une question qui concerne l'ABF.

Aux États-Unis, le paysage est très différent - au point qu'on peut se demander si la défense de la liberté d'information n'est pas l'activité la plus importante de l'ALA, celle qui mobilise le plus d'énergie(s). Pourtant, même si elle est aujourd'hui la valeur matricielle de l'ALA, l'histoire nous apprend que la liberté d'information n'a pas toujours été au cœur des préoccupations ni des bibliothécaires américains, ni de l'ALA. Et c'est logique : former les citoyens et " américaniser » les immigrants ont été $\mathrm{au} \mathrm{XIX}^{\mathrm{e}}$ siècle et dans le premier $\mathrm{XX}^{\mathrm{e}}$ siècle des activités centrales des bibliothèques. Activités qui appelaient une fonction de guide, d'orientation, de conseil. Paternalisme et censure ont été, de pair, les compagnons des bibliothécaires.

Dans les années 1930, changement de paradigme : avec la guerre idéologique contre les totalitarismes européens, la liberté d'information, l'accès à l'information, la circulation des idées, le respect des textes, des œuvres, des livres deviennent primordiaux. En Allemagne, on brûle des livres. L'Amérique doit, au contraire, mettre en avant la liberté des citoyens d'avoir accès à toute l'information - librement. On se souvient des phrases de Franklin D. Roosevelt prononcées en 1942 : « Les bibliothèques sont directement et immédiatement impliquées dans le conflit et ceci pour deux raisons : d'abord, parce qu'elles sont essentielles au fonctionnement d'une société démocratique ; ensuite parce que le conflit actuel concerne l'intégrité du savoir, la liberté intellectuelle et même la survie de notre culture et que les bibliothèques sont de grands outils de savoir, de grands dépôts de culture et de grands symboles de la liberté intellectuelle. ${ }^{319}$ »

\footnotetext{
318. Bulletin d'informations de l'ABF, $\mathrm{n}^{\circ} 176,1997$. Le groupe régional « Paris » rassemble principalement des bibliothécaires des bibliothèques d'étude et de recherche (notamment la BN) et est réputé être de droite ; le groupe régional "Ile-de-France » rassemble principalement des bibliothécaires des bibliothèques publiques de la banlieue parisienne et est réputé être de gauche.

319. Citées par Sidney Ditzion, Arsenals for a Democratic Culture, op. cit. (exergue).
} 
C'est donc ce contexte qui a mené l'ALA à adopter, en 1939, son premier texte sur la liberté d'information : le Library Bill of Rights, inspiré d'un texte adopté par la Public Library de Des Moines. Ce texte révisé et complété de plusieurs autres textes constitue aujourd'hui l'arsenal de l'ALA, la référence aussi bien contre la censure politique, celle de MacCarthy ou celle de gouvernements fédéraux ultérieurs, que contre la censure plus ordinaire que tentent d'instaurer des groupes de pression, de simples usagers ou même des Library Boards.

$\mathrm{Au}$ cours du dernier demi-siècle, l'ALA a créé un outillage riche et complexe appuyé sur de nombreux textes de référence et sur des actions symboliques. La stratégie initiale est de lier la liberté de l'information et la reconnaissance de l'expertise des bibliothécaires. C'est parce qu'ils procurent (qu'ils savent procurer) aux citoyens américains les textes aptes à forger leurs propres convictions que les bibliothécaires travaillent à la fois pour la vie démocratique du pays et pour leur reconnaissance professionnelle - double enjeu, double gain. L'année suivant l'adoption du Library Bill of Rights, en 1940, le Conseil de l'ALA décide la création d'un comité chargé de suivre ces dossiers. Aujourd'hui connu sous le nom de Intellectual Freedom Committee, il s'est d'abord appelé d'un nom plus explicite : le « comité de la liberté d'information pour protéger les droits des usagers des bibliothèques à la libre investigation ${ }^{320}$ ». Ce comité va avoir pour tâche de former les bibliothécaires à leur nouveau credo puis, dans un deuxième temps, d'élaborer les outils propres à lutter contre la censure et à garantir la liberté d'information.

C'est la fin des années 1960 qui marque une deuxième étape dans la lutte contre la censure. En 1967, parmi les neuf objectifs (Goals for Action) définis par l'ALA, figure celui d'« obtenir l'engagement de tous de soutenir et développer les principes du Bill of Rights et du texte Freedom to $\operatorname{Read}^{321}$ ». La même année, après quelques tergiversations, est créé à l'ALA un nouveau bureau pour s'occuper de ce secteur, l'Office for Intellectual Freedom. En 1969, dans le tourbillon qui agite alors l'ALA, il est décidé de soutenir les bibliothécaires attaqués parce qu'ils défendent la liberté d'information, de les soutenir non seulement en mots mais financièrement : c'est la Freedom to Read Foundation qui est créée pour assumer ce rôle. Enfin, en 1973, est créé un groupe de travail, Intellectual Freedom Round Table, qui aujourd'hui, avec plus de 2000 membres, est le plus important

320. Committee on Intellectual Freedom to Safeguard the Rights of Library Users to Freedom of Inquiry.

321. Minutes of the Meeting of the ALA Council. 1967 Annual Conference, San Francisco. 
de l'ALA. Au début des années 1970, l'ALA dispose donc de textes de référence (Library Bill of Rights, Freedom to Read Statement), d'un comité de réflexion (Intellectual Freedom Committee), d'un bureau avec du personnel pour agir (Office for Intellectual Freedom), d'un groupe de travail pour veiller et mobiliser les énergies (Intellectual Freedom Round Table) et d'une fondation pour financer les dépenses nécessaires (Freedom to Read Foundation). Les outils, dès lors, vont se multiplier : création de la Banned Books Week (semaine des livres censurés), appui aux bibliothécaires attaqués, soutien juridique, publication régulière de la liste des livres censurés, témoignages devant les tribunaux ou les commissions d'enquête, campagnes d'opinion, tel Your Right to Know en $1992{ }^{322}$, etc.

De multiples épisodes jalonnent la longue histoire de la lutte de l'ALA pour la liberté d'information. Période dramatique que celle du maccarthysme et du comité contre les « activités non américaines » (Un-American Activities Committee) - les enquêtes de moralité feront perdre leur emploi à des dizaines de bibliothécaires ; des livres, des revues, des collections, les bibliothèques scolaires en particulier seront attaqués comme faisant le jeu de l'ennemi ; de nombreux auteurs sont interdits de rayonnages. L'ALA fait alliance avec l'Association des éditeurs américains (American Book Publishers Council) d'abord pour défendre les bibliothèques des postes diplomatiques, attaquées par MacCarthy, puis pour élaborer et adopter un texte-manifeste, le Freedom to Read Statement.

L'adoption de ce texte reçoit le renfort inestimable du président Eisenhower : huit jours avant la conférence annuelle de l'ALA, le 14 juin 1953, à l'université de Dartmouth, il prononce des phrases limpides contre la censure. " Ne rejoignez pas les brûleurs de livres ». "Allez dans les bibliothèques et lisez-y ce que vous voulez ». "Refuser une place aux idées qui ne sont pas les vôtres est contraire à l'esprit américain ${ }^{323}{ }$ ". Le président envoie une lettre de soutien à l'ALA et la conférence annuelle fut dès lors l'occasion de l'adoption enthousiaste du Freedom to Read Statement * qui rappelait les responsabilités des éditeurs et des bibliothécaires dans la diffusion des idées et défendait vigoureusement la liberté

\footnotetext{
*. Texte en annexe.

322. «Beaucoup de gens ne font pas le lien entre les bibliothécaires et leur droit de savoir. Ils ne se rendent pas compte qu'ils ont accès à un professionnel de l'information et à un important et pertinent gisement d'information dans leur bibliothèque locale. Ils ne comprennent pas davantage le rôle du bibliothécaire qui lutte contre la censure et s'assure que l'information importante (les documents du gouvernement, l'information sur la santé ou l'environnement, comment trouver du travail, etc.) est accessible à tous » (Your Right to Know: Librarians Make it Happen. Celebrating Libraries, Librarians and the Right to Know. ALA Library Campaign Book, ALA, 1992)

323. Louise Robbins. "Champions of a Cause", op. cit.
} 
d'expression : « Nous n'exprimons pas ces propositions en nous abritant derrière l'idée rassurante que ce qui se lit n'a aucune importance. Nous croyons au contraire à l'importance de ce qui se lit. Nous croyons que certaines idées sont dangereuses mais que la censure de ces idées serait un coup fatal porté à la démocratie. La liberté est un mode de vie risqué mais c'est le nôtre. »

Moins aiguë, plus chronique, la menace sur les bibliothèques scolaires ou les sections jeunesse des bibliothèques publiques. À côté de cas fréquents, semble-t-il, d'auto-censure, des attaques venant de l'extérieur tentent de faire enlever des livres des collections. Relayées ou contrées par le Library Board, elles portent sur des livres (ou des films) jugés obscènes ou ayant un vocabulaire offensant ou insultant pour une partie de la population desservie. On cite souvent Huckleberry Finn, censuré parce qu'il emploie le terme "Negroes », L'attrape-cœurs, au vocabulaire trop vulgaire aux yeux de certains, ou la série des Harry Potter critiquée car elle montre la sorcellerie sous un jour positif. Les livres d'éducation sexuelle pour enfants ou adolescents sont, bien sûr, en première ligne.

Dans un autre registre, l'arrivée d'Internet, particulièrement dans les Public Libraries, relance la question de la censure : dans ces endroits publics, désormais largement et très publiquement ancrés dans la lutte contre toute restriction d'accès, les enfants peuvent-ils tout voir ? Ou, plus exactement, voir n'importe quoi ? Qui a la responsabilité de veiller à ce qu'ils ne voient pas n'importe quoi quand ils viennent à la bibliothèque ? Les parents, répondent l'ALA. Les bibliothécaires, répond le gouvernement : il existe des outils (les filtres) pour éviter que des images choquantes, pornographiques, violentes, ne soient visibles sur un écran d'ordinateur, même accidentellement. La crainte devant la diffusion (volontaire ou non) d'images violentes ou « indécentes » par le biais de ces nouveaux canaux engendre une cascade de textes : le Child Pornography Prevention Act (CPPA) en 1996, le Child Online Protection Act (COPA) en 1998 et le Children's Internet Protection Act (CIPA) en décembre 2000. Pour faire pression sur les bibliothèques (scolaires et publiques), le gouvernement décide que l'aide fédérale ne sera désormais versée qu'à celles qui filtrent Internet.

L'ALA, selon son habitude, entame d'abord une campagne d'opinion. Libraries: An American Value, texte adopté par le Conseil le 3 février 1999, mentionne comme première des valeurs défendues le droit des jeunes à l'usage de la bibliothèque et, plus loin dans le texte, affirme « la respon- 
sabilité et le droit des parents à guider leurs propres enfants dans l'usage qu'ils font de la bibliothèque, de ses ressources et de ses services ».

Comme elle l'avait fait contre le Communications Decency Act de $1996^{324}$, l'ALA choisit la voie judiciaire et décide en janvier 2001 d'aller en justice, soutenue par l'ACLU, pour que soit établie la non-constitutionnalité du CIPA - comme enfreignant le Premier Amendement de la Constitution par le biais du filtrage de tous les postes de la bibliothèque, et pas seulement des ordinateurs destinés aux enfants. Le droit des adultes à la libre information est donc ainsi bafoué. Jack Kessler explicite la radicalité de cette position : «Aux États-Unis comme ailleurs, les bibliothécaires comptent parmi les défenseurs les plus acharnés de ces libertés, mais nulle part plus qu'aux États-Unis ils ne sont aussi militants, voire combatifs, sur la question de la liberté d'expression. En l'occurrence, la plus importante association de bibliothécaires des États-Unis a officiellement porté plainte contre son propre gouvernement : le principal procès amené par la nouvelle loi sur la censure va en effet concerner "American Library Association versus the United States” ${ }^{325}$ ». En première instance, en avril 2002, l'ALA est déboutée. En appel, en novembre 2002, les juges lui donnent raison. En juin 2003, la Cour suprême lui donne tort : car, entre-temps, le gouvernement a admis que les usagers pouvaient demander à la bibliothèque de retirer les filtres : l'atteinte à la liberté d'information devient (légalement) minime, puisqu'on peut y mettre fin à la demande. Il n'y a donc pas atteinte au Premier Amendement, conclut la Cour suprême par 6 voix contre $3{ }^{326}$. Le débat ne porte plus, dès lors, sur le principe du filtrage mais sur son application.

Dans cette affaire, plusieurs éléments peuvent être mis en évidence en termes de rapport au pouvoir. D'abord, c'est la voie judiciaire qui est privilégiée : c'est de la justice, et en particulier de la Cour suprême, que l'ALA attend satisfaction. Ensuite, parallèlement, le combat politique se mène avec des alliés, au premier rang desquels l'ACLU, mais aussi des groupes de défense locaux et de simples usagers des bibliothèques. Enfin, ce combat pour défendre l'accès de tous à tous les documents (réels ou virtuels) n'est pas consensuel. La position de l'ALA est vue, même par des

324. Procédure engagée avec l'ACLU et gagnée devant la Cour suprême en juin 1997 : la mise en cause et la poursuite des distributeurs et diffuseurs de documents sont déclarées contraires au Premier Amendement (affaire Reno v. American Civil Liberties Union et al.).

325. Jack Kessler. "Tout a changé : le filtrage des informations et la censure, une actualité dans les nouveaux États-Unis d'Amérique », Bulletin des bibliothèques de France, t. 47, n² 2, 2002.

326. < http://www.ala.org/ala/washoff/WOissues/civilliberties/cipaweb/cipa.htm > 
bibliothécaires, comme intégriste et ne remporte pas l'adhésion de l'ensemble du monde des bibliothèques - ni même du monde politique qui lui est habituellement favorable, Hillary Clinton, par exemple, déclarait que si elle travaillait en bibliothèque, elle utiliserait des filtres « car il y a un tas de choses sur le Web qui ne sont tout simplement pas pour les enfants ${ }^{327}$ ".

Un autre combat, encore en cours aujourd'hui, oppose à nouveau l'ALA et le gouvernement fédéral : c'est l'application de l'USA Patriot Act, qui autorise les services de police et de renseignements à obtenir des bibliothécaires la liste de ce que les lecteurs consultent (livres, journaux, films ou sites Internet). Ce combat est beaucoup plus populaire, car il s'agit clairement d'un combat pour les libertés civiles - les bibliothécaires disant aux Américains qu'ils ne doivent pas avoir un « bibliothécaire par-dessus leur épaule », comme mouchard et auxiliaire de la police ${ }^{328}$.

Cet arbre gigantesque qu'est devenue la défense de la liberté d'information, dans le paysage des bibliothèques américaines, interroge évidemment sur sa place par rapport aux autres objectifs qui peuvent être mis en avant - l'égalité d'accès, le rôle des bibliothèques dans un monde démocratique, la transmission du savoir, etc. Cette hypertrophie ouvre sur la question du rôle de l'ALA. Vu d'Europe, en tout cas, on peut se demander si la liberté d'information n'est pas, pour une large part, devenue un objet rhétorique, centré sur lui-même et qui a pour principale qualité d'être consensuel.

Cette question n'obère pas la différence d'activité, de positionnement et d'efficacité entre les associations professionnelles des deux pays - que, encore une fois, la taille ne suffit pas à expliquer.

327. "A lot of stuff on the Web that is just not appropriate for children" (American Libraries on line, 20 march 2000).

328. Documentation abondante sur le site de l'ALA. On peut consulter également Nancy Kranich, "Le USA Patriot Act : conséquences sur la liberté d'expression ", Bulletin des bibliothèques de France, t. 49, n 6, 2004 ou Philippe Cantié, Au nom de l'antiterrorisme : les bibliothécaires américains face à l'USA Patriot Act, Presses de l'enssib, 2006. Dans ce combat, on retrouve la même approbation de la part de Kurt Vonnegut que celle de Howard Fast à propos de lutte contre les censures maccarthystes : "Puisque nous parlons de livres qui brûlent, je tiens à féliciter les bibliothécaires, qui n'ont pas la réputation d'être physiquement forts, de disposer de contacts politiques puissants ou de posséder de grandes fortunes, et qui ont pourtant résisté fermement, dans tout le pays, aux brutes antidémocrates les pressant de retirer certains livres de leurs étagères. Ils ont préféré détruire des fichiers plutôt que de révéler à la police de la pensée les noms des personnes qui avaient consulté ces titres. L'Amérique que j'aime existe toujours, même si elle n'est pas à la Maison-Blanche, à la Cour suprême, au Sénat, à la Chambre des représentants ou dans les médias. L’Amérique que j'aime existe toujours dans nos bibliothèques publiques. » (Kurt Vonnegut. Un homme sans patrie. Denoël, 2006, p. 103). Les bibliothèques, une valeur américaine ! 
CHAPITRE V
DEUX MODELLES DE
BIBLIOTHEQQUE 


\section{CHAPITRE V DEUX MODĖLES DE BIBLIOTHÈQUE}

$\mathrm{L}$ es valeurs, objectifs, combats, débats que nous avons rencontrés, ces histoires ont dessiné deux modèles de bibliothèque qui présentent des caractéristiques pour partie communes, pour partie différentes. Commun(e)s, l'organisation, un grand nombre des services offerts, l'inscription dans un modèle démocratique, le souci de l'égalité d'accès. Différent(e)s, le rapport au public, le rapport au passé (le patrimoine), les collections, l'image de la bibliothèque. Ces ressemblances et ces écarts ont quelque chose à voir avec la politique et la société dans lesquelles les bibliothèques s'inscrivent - comment en serait-il autrement ?

\section{UN SOCLE COMMUN}

Le «modèle américain » a été adopté en France. Il est donc logique que beaucoup des traits des Public Libraries se retrouvent dans les bibliothèques municipales. C'est ainsi que, si l'on remonte au début de notre période, ont été progressivement acclimatés le libre accès aux rayons, les sections enfantines, la presse, les livres pratiques, la littérature non légitime (les bandes dessinées, les romans policiers, les romans de sciencefiction), la musique non légitime (alors que, dans les années 1960, seule «la grande musique » avait droit de cité), le cinéma non légitime (alors que, initialement, seuls les documentaires et les « classiques du cinéma » avaient droit de cité), le souci des publics « empêchés » (handicapés, hospitalisés, détenus, etc.), l'organisation en bibliothèques centrales et annexes, etc. En somme, les éléments qui marquent la rupture avec la bibliothèque savante du XIX siècle. À la fin des années 1970, au début des années 1980, le modèle est peu ou prou adopté. Adopté dans les têtes des responsables politiques, dans les têtes des bibliothécaires et dans les établissements.

Comme les bibliothèques américaines, les bibliothèques françaises accueillent les enfants de plus en plus jeunes et leur proposent l'heure du conte ; elles multiplient les actions " hors-les murs », dans les crèches, les centres aérés, les foyers de personnes âgées ; elles se font conviviales 
en se dotant de cafétérias ou de coins-repos ; elles se modernisent en mettant leur catalogue en ligne ou en offrant des connexions à Internet...

L'organisation par sections, les outils informatiques, les normes et procédures (Dewey, Dublin Core) : les dispositifs rapprochent les établissements. Le vieillissement du groupe professionnel se produit des deux côtés de l'Atlantique ${ }^{329}$, la raréfaction des budgets publics les touche pareillement. Dans les deux pays, on pense que les bibliothèques sont utiles à la société : $98 \%$ aux États-Unis, $75 \%$ en France - l'écart s'expliquant par l'écart de fréquentation réelle et donc par une utilité effective là-bas, une utilité supposée ici.

Pourtant, les différences sont sensibles - et pas seulement dues à des effets de taille. Je vais insister ici sur trois registres dans lesquels ces différences sont manifestes : les publics, les collections, l'image de la bibliothèque.

\section{LES BIBLIOTHÈQUES ET LEURS PUBLICS}

J'ai évoqué plus haut les fondements politiques des bibliothèques, leur implantation dans leur territoire, leur rapport à la citoyenneté, à la vie démocratique de leur pays. Cette analyse des valeurs doit être complétée, nuancée de l'analyse des activités et des réalisations.

Rappelons une dernière fois quelques chiffres. Il existe environ 9200 Public Libraries aux États-Unis, environ 3000 bibliothèques municipales en France. Environ les deux tiers de la population fréquentent une Public Library, environ $25 \%$ une bibliothèque municipale. Ces chiffres ne sont connus que par sondages, les statistiques annuelles étant établies aux États-Unis sur les entrées (1,2 milliard en 2001) et en France sur les inscrits (6 740000 en 2004, soit 17,5\% de la population desservie). Les chiffres américains sont stables à un bon niveau, les chiffres français sont imprécis, certaines sources (la Direction du livre et de la lecture) annonçant une fréquentation en baisse, alors qu'une étude menée par le CREDOC en 2005 annonce des chiffres en hausse ${ }^{330}$.

329. Françoise Gaudet, Claudine Lieber. « De l'autre côté de l'Atlantique : recruter ou périr ?». Bibliothécaire, quel métier?, sous la dir. de Bertrand Calenge. Éditions du Cercle de la Librairie, 2004 (Bibliothèques).

330. La stagnation des chiffres n'est pas une fatalité : entre 1994 et 2004, la proportion de Québécois fréquentant une bibliothèque publique est passée de $32 \%$ à $47 \%$.

< http://www.stat.gouv.qc.ca/observatoire/ > 
Dans leurs statistiques d'activités ${ }^{331}$, les Américains comptent la population desservie, les entrées, les prêts (adultes et enfants), le public des animations pour enfants, les accès à Internet, les questions posées (reference transactions ${ }^{332}$ ), les prêts entre bibliothèques. Les Français comptent la population desservie, les inscrits, les prêts, les accès à Internet et les horaires ${ }^{333}$. Où l'on voit déjà que l'accent n'est pas mis tout à fait sur le même registre puisque l'on ne décompte pas les mêmes activités.

\section{Éduquer}

À travers les décennies, on l'a vu, la fonction éducative de la Public Library est magnifiée. Ne l'appelle-t-on pas «l'université du peuple » ${ }^{334}$ ? L'éducation des adultes est une mission des Public Libraries depuis le XIX ${ }^{e}$ siècle, chargées qu'elles étaient de former les citoyens, instruire les ouvriers et « américaniser » les immigrants.

Cette image, toujours vivace, expliquerait le succès des Public Libraries encore aujourd'hui : "Pourquoi cette institution est-elle si répandue aux États-Unis ? La réponse doit être que, en dépit du faible usage qu'en font réellement les adultes, la Public Library a toujours mis l'accent sur son caractère d'institution éducative. ${ }^{335}$ "

Par le biais de la collecte statistique, on l'a vu, se retrouve une des différences majeures entre les Public Libraries et les bibliothèques municipales : le caractère éducatif de l'institution. Le comptage des questions posées mesure l'activité documentaire et éducative de la bibliothèque. Ce comptage n'est pas fait en France. Faut-il souligner que la fonction même de bibliothécaire de référence (reference librarian, qui répond aux questions des usagers) n'existe pas dans les bibliothèques municipales françaises ? Elle est pourtant très présente dans les Public Libraries, même dans les petites villes.

Partout, les enfants viennent faire leurs devoirs, des bénévoles les y aident. Les teen-agers sont une cible prioritaire des bibliothèques

331. Public Libraries in the United States: Fiscal Year 2001, NCES, 2003.

[En ligne] < http://nces.ed.gov/surveys/libraries >

332. Il ne s'agit pas là d'un lointain héritage : ces données ne sont collectées que depuis 1977.

333. Direction du livre et de la lecture. Bibliothèques municipales, bibliothèques départementales des départements d'outre-mer, bibliothèques départementales de prêt : données 2002, ministère de la Culture, 2004.

334. “The People's University”. Formule de Alvin Johnson.

335. Lawrence White. The Public Library in the 1980s: The Problems of Choice. Lexington Books, 1983, p. 137. 
aujourd'hui ${ }^{336}$. La Public Library est vue comme une deuxième école, après l'école. Elle participe activement à la «formation tout au long de la vie », réactivant sous de nouvelles modalités l'idée d'autodidaxie : «Tous ceux qui le souhaitent - et beaucoup l'ont souhaité - peuvent s'éduquer euxmêmes grâce à la Public Library ${ }^{337}$ ». Elle propose, partout, des cours d'anglais pour les immigrants. Les grandes bibliothèques ont des départements « recherche » et servent de bibliothèques de recours pour les plus petites.

La devise de la Chicago Public Library est " Lisez, apprenez, découvrez ! ». Son Customer Bill of Rights (charte de l'usager) commence ainsi : "Vous avez le droit de

- recevoir un traitement courtois

- demander de l'aide à un bibliothécaire

- appeler ou venir pour avoir accès au service de référence [...] ».

En 1999, la Salt Lake City Public Library a inventé une assistance à la maîtrise de la lecture : lire à un chien. Farfelu ? Le chien, expliquent les bibliothécaires, ne reprend pas les enfants, ne se moque pas d'eux, ne trouve pas qu'ils lisent trop lentement : il leur donne confiance ${ }^{338}$. La Champaign Public Library (Illinois) organise (comme beaucoup d'autres...) tous les étés des ateliers de lecture ou des concours : "Sois un super lecteur cet été ! » : après la lecture de 5 livres, l'enfant reçoit un cadeau, au $15^{\mathrm{e}}$ livre on lui offre un livre, au $25^{\mathrm{e}}$ il reçoit un " T-shirt de super héros ».

En 2005, la campagne « Every Child Ready to Read » de l'ALA affiche comme premier argument : " Les bibliothèques aident les élèves à mieux travailler à l'école ». Cette phrase ne peut que susciter l'incompréhension, voire l'hostilité, des bibliothécaires français pour qui la bibliothèque et l'école occupent deux territoires distincts, défendent deux conceptions du livre et de la lecture et pour qui, en aucun cas, la bibliothèque ne doit être un substitut de l'école.

" Il faut se rendre compte », analyse Martine Blanc-Montmayeur, à l'époque directrice de la BPI, « que l'ensemble de la profession des bibliothécaires de lecture enfantine avait été formé [dans les années 1970 et 1980] au sein de la Joie par les livres dans une idéologie qui était de faire tout ce que ne faisait pas l'école, et en aucun cas de s'identifier à l'école. La bibliothèque enfantine devait être le lieu de la culture-plaisir non obliga-

336. Les enfants représentaient $37 \%$ des usagers, les 12-18 ans $23 \%$ en 1993 (enquête NECS). 337. Ernestine Rose. The Public Library in American Life. Columbia University Press, 1954, p. 122. 338. Le Monde, 5 février 2006. 
toire, non liée à des programmes scolaires. ${ }^{339}$ » Elle voit un changement d'attitude dans les années 1990, sous l'effet de "l'invention de l'illettrisme » et de l'échec scolaire, les bibliothèques enfantines revisitant leurs politiques d'achat pour renforcer leurs collections de livres documentaires, voire acheter des manuels, renforçant leur collaboration avec les bibliothèques scolaires et introduisant l'aide aux devoirs.

Symétriquement, souligne Max Butlen, les pratiques de lecture à l'école sont influencées par les bibliothèques jeunesse : « Les instituteurs militants à la recherche d'une nouvelle pédagogie de la lecture, les chercheurs et les bibliothécaires jeunesse les plus impliqués se sont logiquement rencontrés et se sont entendus sans trop de difficultés pour améliorer une offre de lecture scolaire qui leur paraissait désuète, inadaptée, inefficace. De cette union est né un nouveau modèle de bibliothèque, la bibliothèque centre documentaire qu'ils ont tenté d'implanter à l'école, avec une double volonté : "ni scolariser la bibliothèque enfantine ni remplacer l'école". ${ }^{340}$ " Ce rapprochement a connu un apogée tourmentée avec l'adoption des programmes scolaires de 2002, où la littérature pour la jeunesse est devenue partie intégrante des programmes scolaires ${ }^{341}$.

Même s'ils sont, on le voit, plus complexes que l'on ne le dit, les rapports des bibliothécaires et des enseignants sont, en France, depuis longtemps, tendus, marqués par des désaccords et la volonté de se distinguer les uns des autres. "Les bibliothèques pour enfants réclament de demeurer du côté de la séduction », résume Hélène Weis ${ }^{342}$.

Au-delà de l'accompagnement des enfants, la fonction éducative en direction des adultes est assumée avec la même réticence. Si le vieux concept d'éducation populaire n'a pas disparu, il a évidemment évolué avec l'élévation du niveau scolaire moyen de la population et les nouveaux visages de l'autodidaxie. Cette préoccupation n'a pas disparu de l'horizon des bibliothécaires français, à l'instar de Jean-Pierre Seguin, concepteur de la BPI qu'il décrivait comme « une bibliothèque universi-

339. Martine Blanc-Montmayeur. "Le rôle social de la bibliothèque : quelles significations ? " Regards croisés et perspectives : les bibliothèques publiques en Europe, BPI, 2000, p. 77.

340. Max Butlen. "Lire en bibliothèque, lire à l'école : oppositions et interactions ». Bulletin des bibliothèques de France, t. 49, nº 1, 2004.

341. Les débats se sont cristallisés sur la liste des ouvrages susceptibles d'entrer à l'école dans le cadre de ces programmes : «La mise en cause de cette liste avec ses péripéties, ses rebondissements a été un bon révélateur tout à la fois des limites du consensus autour de l'offre, des rapports de force et des tensions qui résultent de la concurrence entre acteurs résolus à faire prévaloir leurs valeurs, leurs représentations, leur politique d'offre et/ou leurs intérêts ", analyse Max Butlen (ibid.).

342. Hélène Weis. « Les bibliothèques pour enfants en quête d'un nouveau modèle ». Regards sur un demi-siècle, op. cit., p. 177. 
taire pour non-universitaires ». Mais les étudiants, en bibliothèque municipale, sont souvent vus comme des usagers abusifs, déplacés - et l'ABF cherche à lutter contre cette tendance : "Pourquoi nombre de bibliothèques municipales cherchent-elles à décourager le public étudiant, réputé être un "mauvais public" : consommateur d'espace, exigeant sur le plan des documents, mais faible emprunteur, soupçonné de peu de considération pour le bien public ? N'est-ce pas pourtant un des publics qui ont un besoin très légitime des services de la bibliothèque ? ${ }^{343}$ "

Pourtant, les étudiants semblent demeurer un poids pour les bibliothécaires français, car trop nombreux, trop présents, accaparant les espaces et les services au détriment du public ordinaire.

La démocratisation culturelle, l'ouverture à tous les publics semblent être, pour les bibliothécaires, distinctes du partage du savoir. C'est un des constats du travail de Nicolas Beudon : " Le vaste mouvement de modernisation des bibliothèques publiques qui s'est enclenché dans les années 60 s'est construit davantage sur l'idée d'une démocratisation de la culture, voire même des loisirs, plutôt que des savoirs. ${ }^{344}$ »

Pourtant, les promoteurs du modèle de bibliothèque publique vont plaider en faveur de ce rôle éducatif. Jean Hassenforder « compte parmi les passeurs qui vont s'efforcer de trouver une traduction dans le monde des bibliothèques au modèle de l'autodidaxie à l'anglosaxonne et aux slogans de l'éducation permanente. Avec ses amis de la section des petites et moyennes bibliothèques à vocation éducative, qui bouscule l'ABF à sa création en 1959, il ambitionne de définir un nouveau modèle de bibliothèque [...]. Sa “vocation éducative”, inspirée par l'idée de culture générale portée par la formation permanente, implique de rompre en partie avec une "culture traditionnelle à dominante littéraire, artistique, historique”. Pour Hassenforder en effet "la bibliothèque moderne devient un centre documentaire." ${ }^{345}$ »

« La bibliothèque municipale n'a pas à jouer le rôle de centre de documentation », proteste en écho Noë Richter en 1965. Trente ans plus tard, même protestation : " Il existe, en bien des établissements, une possible contamination du service lecture publique par celui d'études ou de référence. Faut-il une politique draconienne pour éviter la fusion des deux et

343. Jean-François Jacques. «Publics et services ». Le métier de bibliothécaire, op. cit., p. 308.

344. Nicolas Beudon. Apprendre et se former dans les bibliothèques : la mission éducative des bibliothèques municipales, Mémoire d'étude du diplôme de conservateur des bibliothèques : enssib, 2008. 
protéger le premier comme une espèce en voie de disparition ? [...] Dans la médiathèque, devenue pour l'essentiel une auxiliaire de l'école, la place des animations sera-t-elle à réétudier ? Ne ferons-nous bientôt que des expositions sur les écrivains du bac ? ${ }^{346}$ "

Malgré l'influence anglo-saxonne, les bibliothèques municipales, décidément, hésitent, répugnent à être considérées comme des institutions éducatives.

\section{Accueillir les individus}

Les visiteurs des bibliothèques américaines soulignent volontiers la qualité de l'accueil que les usagers y reçoivent. C'était déjà le cas du Norvégien Axel Munthe, à son retour d'Amérique, en 1939, qui saluait « ce charmant mélange de sollicitude, de patience et de courtoisie, et cette compréhension souriante des souhaits du public, même inexprimés, qui sont la note dominante de l'établissement tout entier et qui ont rendu les bibliothèques américaines sans rivales à travers le monde. ${ }^{347}$ "

En 1954, Ernestine Rose parlait de bibliothécaires faisant preuve d'un « intérêt amical envers les gens ». Dans un film réalisé en 1992, à l'occasion du centenaire de la New York Public Library, "The People's Palace », Toni Morrison parle de cette bibliothèque comme d'un " modèle pour toutes les bibliothèques du pays : pas nécessairement par sa taille, mais par l'attitude de son personnel », qui guide, explique, répond... Les différences dans l'accueil des publics ne passent donc pas seulement par le plus évident, le plus débattu, l'accueil des publics spécifiques, la question des communautés, mais d'abord par le sens de l'accueil - dont on accuse régulièrement les bibliothécaires français d'être dépourvus ${ }^{348}$.

La pratique du eye contact, le Can I help you? contraste avec le nez baissé et l'évitement trop souvent constatés aux bureaux d'accueil en France. C'est que la culture professionnelle française continue à valoriser les tâches scientifiques ou techniques (le choix des acquisitions, l'indexation...) plus que les tâches d'accueil et de renseignement des usagers. Ainsi, une directrice de bibliothèque en vient à dire qu'un bibliothécaire

346. Jean-Claude Le Dro, « Les exclus de la médiathèque », Bulletin d'informations de l'ABF, $\mathrm{n}^{\circ}$ 170, 1996.

347. Axel Munthe, American Librarianship from an European Angle: An Attempt at an Evaluation of Politics and Activities, ALA, 1939, p. 159.

348. Par exemple : "Cette profession, qui parle tant de la fonction de renseignement, continue à ignorer ou dévaloriser celle d'accueil. » (Jean-Claude Utard « Le succès des bibliothèques publiques », Bulletin des bibliothèques de France, t. 42, $\mathrm{n}^{\circ} 4$, 1997). 
non performant à l'accueil ne peut en être enlevé car ce serait lui faire une faveur injustifiée ${ }^{349}$. Symétriquement, l’ABF plaide pour que ces tâches d'accueil soient considérées comme essentielles : "Compte tenu des moyens informatiques dont disposent aujourd'hui les bibliothèques, de la possibilité de récupérer les notices bibliographiques, et des divers procédés d'aide à la communication et au travail, il n'est guère réaliste de prétendre que les bibliothécaires doivent passer en travail interne plus de la moitié de leur temps de travail. Le métier doit d'abord être considéré comme un métier de contact et d'accueil, et cette activité doit être prioritaire, quel que soit le type de bibliothèque. ${ }^{350}$ » Si cette affirmation est encore nécessaire aujourd'hui, c'est bien qu'elle n'est pas unanimement partagée...

Un autre versant de l'accueil est la question des horaires. En France, la moyenne est bloquée à un chiffre dérisoire (19 h 30 en 2005), mais avec des nuances importantes : les horaires moyens diminuent avec la taille de la ville. La moyenne hebdomadaire est de plus de 42 heures pour les villes de plus de 300000 habitants, de 39 heures entre 100000 et 300000 habitants, de 32 heures entre 50000 et 100000 habitants et passe sous la barre des 30 heures en dessous de ce seuil. L'ouverture du dimanche fait l'objet de débats âpres entre bibliothécaires, partagés entre le souci de desservir plus efficacement la population, notamment en ouvrant aux heures où elle est disponible, entre l'intérêt des personnels (souvent réticents à sacrifier le repos dominical et familial) et la volonté de s'afficher comme un service non marchand et, donc, insensible aux sirènes de la consommation qui poussent à l'ouverture du dimanche. L'équilibre entre ces trois arguments aboutit, aujourd'hui, à ce qu'un très petit nombre de bibliothèques municipales ouvre le dimanche - alors que c'est la norme aux États-Unis.

L'accueil, aujourd'hui, se fait non seulement sur place mais à distance. La New York Public Library annonçait, dès 2001, que son site Web était désormais plus fréquenté que ses locaux. Quant à la place même d'Internet dans les bibliothèques, elle connaît d'une part une différence d'intensité (de calendrier, sans doute) : les Public Libraries ont commencé plus tôt que les bibliothèques municipales à proposer un accès public (et gratuit) à Internet (en 2000, 85 \% des Public Libraries proposaient déjà ce ser-

\footnotetext{
349. « Donc, je ne peux pas dire à quelqu'un : ne faites plus de service public. Ne faites que du service intérieur. Ça serait à la limite, vis-à-vis du reste de l'équipe, lui accorder un privilège... parce que c'est fatigant de faire du service public. » (citée dans Anne-Marie Bertrand, Bibliothécaires face au public, BPI, 1995, p. 198)

350. Jean-François Jacques. « Publics et services », op. cit., p. 311.
} 
vice), les premières bibliothèques municipales ont commencé il y a une petite dizaine d'années et, estime-t-on, la moitié d'entre elles aujourd'hui propose ce service. Elle connait d'autre part une différence dans l'offre, notamment par le biais du site Web de la bibliothèque. Les Public Libraries proposent très souvent un service de réponses à distance (Ask a Librarian où les questions et les réponses sont transmises par messagerie électronique, voire par chat), organisé dans le réseau collaboratif et qui est donc un nouvel avatar de la bibliothèque comme lieu de ressources documentaires. Elles proposent d'autres services, étonnants pour le public français : ainsi, dès la page d'accueil, la possibilité de soutenir financièrement la Public Library, les activités pour les teens ou des coups de cœur de lecture (proposition exceptionnelle en France). En France, c'est souvent le catalogue de la bibliothèque et l'annonce des manifestations culturelles qui sont les uniques propositions que l'on peut trouver sur le site des bibliothèques - à l'exception de quelques cas dont la Bibliothèque municipale de Lyon est l'exemple le plus abouti, avec ses informations sur l'activité de la bibliothèque, le catalogue en ligne, un service de questions-réponses (le Guichet du savoir), des expositions en ligne ou les coups de cœur des bibliothécaires qui, contrairement à la plupart de leurs collègues en France, acceptent de prendre parti et de conseiller certains ouvrages.

Du côté français, on retrouve ici la même réticence à l'accueil, la même faible culture de service : en 2005, des débats ont eu lieu non sur la possibilité (réelle) mais sur la légitimité d'offrir aux usagers la possibilité de réserver un ouvrage en ligne. À nouveau est apparue la faible culture de service : permettre aux usagers de réserver un document à distance ne serait que pénaliser les usagers qui viennent le chercher sur place - argument dont la pertinence n'est pas évidente et qui manifeste surtout cette vieille idée que " la culture, ça se mérite » et que les efforts consentis sont la marque de l'adhésion à la culture de l'institution ${ }^{351}$.

Internet va-t-il conduire à la mort des bibliothèques ? Ici, de longs développements seraient nécessaires sur l'offre numérique (ce qu'on trouve sur Internet, la fiabilité des informations, leur pérennité, leur coût) et sur la demande (professionnelle, scolaire, de loisirs). Toute une littérature navigue entre « l'extase » et «l'effroi »: l'extase car tout deviendrait accessible pour tout le monde, l'effroi car ce serait l'apothéose de la culture-

351. Sur ce point, je me permets de renvoyer à mon ouvrage, Anne-Marie Bertrand, Bibliothécaires face au public, op. cit., p. 117-128. 
zapping. Après des années d'usage, la modération semble de retour et les jugements à l'emporte-pièce (ou les signes de panique) semblent s'effacer. Et pour les usagers ? Aux États-Unis, le rapport Benton ${ }^{352}$ souligne que l'âge numérique a entraîné une désaffection de la part des jeunes (18-24 ans) qui pensent que la bibliothèque dans trente ans sera devenue une espèce de musée dont des retraités pourront s'occuper. "Assertions spécieuses », répond Michael Gorman qui met en doute la fiabilité de l'enquête, alors que Kathleen de la Peña McCook proteste que les bibliothécaires interrogés ne reflètent l'opinion que de « la classe moyenne blanche ».

Au-delà des représentations, les usages semblent se déplacer : la fréquentation des bureaux d'information est à la baisse. Françoise Gaudet et Claudine Lieber analysent cette baisse comme une autonomie plus grande des usagers pour les questions simples auxquelles ils trouvent réponse sur Internet: " C'est un phénomène national qui touche jusqu'à la Bibliothèque du Congrès, déclare Roberto Esteves. À Santa Monica, Nancy 0'Neill estime que les questions sont moins nombreuses, mais qu'elles sont plus compliquées : il semble que les usagers se débrouillent maintenant par eux-mêmes lorsqu'ils sont à la recherche d'informations simples à trouver. ${ }^{353}$ » Parallèlement, les renseignements à distance par téléphone et surtout par Internet (notamment le service Ask a Librarian) augmentent leur flux. Il y aurait donc bien une modification des usages plus qu'une diminution des usages. C'est ce qui semble rassurer les bibliothécaires américains qui glosent abondamment sur cette question - l'ALA, bien entendu, n'est pas en reste et diffuse les « Dix raisons pour lesquelles Internet ne se substitue pas à la bibliothèque ».

En France, le renseignement à distance commence tout juste à être mis en œuvre - depuis 2004 à la Bibliothèque municipale de Lyon qui est la première à offrir ce service. Cette offre nouvelle se veut complémentaire de l'offre physique de la bibliothèque. La première année, ce service à distance, le Guichet du savoir, générait une vingtaine de questions par jour ; ses usagers sont à $40 \%$ des personnes qui ne sont jamais venues à la bibliothèque "physique » : il s'agit donc bien (au moins pour partie) d'une offre complémentaire et non substitutive de l'offre traditionnelle.

352. Financé par la Benton Foundation, ce rapport compare les attentes de la population et la vision des bibliothécaires, "Buildings, Books and Bytes: Libraries and Communities in the Digital Age", Library Trends, Summer 1997.

353. Françoise Gaudet, Claudine Lieber. "L’Amérique à votre porte : nouveaux usages, nouveaux services, nouveaux concepts de bâtiments... un avant-goût de notre avenir ? », Bulletin des bibliothèques de France, t. 47, n 6, 2002. 
C'est logique : aux États-Unis, où les Public Libraries ont fait le plein de leurs usagers, Internet incite à un transfert d'usage ; en France, où c'est loin d'être le cas, il s'agit d'une augmentation de l'usage...

\section{Accueillir les communautés}

La question des minorités a pris une acuité particulière dans les années 1960 aux États-Unis, dans les années 1980 en France. Elles sont traitées différemment parce que les fondements politiques sont, sur ce point, différents : la République française est une et indivisible, ne connaît pas, ne veut pas connaître les origines culturelles, ethniques, religieuses de ses citoyens ${ }^{354}$ - la légitimité des enquêtes prenant ces éléments en compte est encore aujourd'hui problématique.

Dans l'organisation de la vie politique, les groupes, les états, les corporations, les associations sont considérés comme des niveaux d'organisation normaux aux États-Unis alors que le rapport direct entre le citoyen et l'État est la norme en France. « À la valorisation des groupes intermédiaires, bases de l'organisation démocratique, s'oppose la vision selon laquelle il n'y a rien entre le citoyen et l'État, entre la volonté individuelle et la volonté générale. Dans certaines démocraties, tels les États-Unis, l'association est célébrée ( $a$ nation of joiners), dans d'autres, telle la France, les groupes sont considérés comme malfaisants ${ }^{355}$ ».

Aux États-Unis, le patchwork des minorités a éclaté au grand jour dans les années 1960, avec la lutte pour les droits civils, les émeutes raciales, les athlètes noirs levant le poing sur le podium olympique de Mexico en 1968. L'Affirmative Action, pour être organisée et évaluée, a besoin de connaître les caractéristiques des citoyens.

Pays jadis esclavagiste, pays toujours d'immigration, les États-Unis ont toujours vécu avec la conscience (plus ou moins malheureuse) de ses minorités. Dans les Public Libraries, la prise en compte de ces minorités a connu un tournant dans les années 1960. On en voit un exemple chez Lowell Martin, dans son étude sur la Chicago Public Library : le projet est "de toucher la population de la ville dans toute sa diversité plutôt que d'attendre d'elle qu'elle se conforme à un modèle standardisé. » L'ALA, en

354. À propos de la formule « le peuple corse », le Conseil constitutionnel rappelle en 1991 que la «Constitution ne connaît que le peuple français, sans distinction d'origine, de race ou de religion » (cité par Dominique Schnapper, La démocratie providentielle : essai sur l'égalité contemporaine, Gallimard, 2002, p. 201)

355. Yves Mény, Yves Surel. Politique comparée : les démocraties, Allemagne, États-Unis, France, Grande-Bretagne, Italie. Montchrestien, $6^{e}$ ed., 2001, p. 10. 
1972, fait le même raisonnement : " Le rôle culturel de la Public Library s'est déplacé de l'image de la Public Library du XIX siècle, instrument d'acculturation, d'américanisation et de standardisation des styles de vie et des valeurs, vers une institution qui reconnaît les différences culturelles et ethniques et encourage la fierté de soi et la reconnaissance des différents patrimoines culturels. ${ }^{356}$ "

Chacune des Public Libraries, dans son territoire, prend en charge cette question, aidée par la politique de planification et programmation : pour planifier, expliquent à l'envi les manuels, il faut bien connaître la communauté à desservir. Cette connaissance de la communauté à desservir facilite l'émergence des minorités. Minorités qui n'ont pas exactement les mêmes contours qu'en France : on cite bien sûr les minorités ethniques, les Noirs, les Latinos, mais aussi les primo-immigrants qui ont besoin d'apprendre l'anglais (et leur nouveau pays, et leurs droits), mais aussi les enfants, les personnes âgées, les handicapés, les surdoués, les habitants des zones isolées, les étudiants pauvres, etc. La complexité de cette approche se retrouve sous la plume de Lowell Martin qui assigne comme mission à la Chicago Public Library de desservir les « centaines de milliers de personnes instruites, bien cultivées, cosmopolites [...] pour qui les bibliothèques de quartier et leurs collections de base ne conviennent pas » aussi bien que " les centaines de milliers de personnes qui commencent juste à utiliser le document imprimé et pour qui les bibliothèques de quartier de base ne conviennent pas non plus. ${ }^{357}$ "

En France, les minorités reconnues par les bibliothèques, histoire politique oblige, ne sont pas ethniques mais économiques (les « personnes défavorisées »), socio-culturelles (les personnes « éloignées du livre ») ou statutaires (les publics "empêchés", personnes hospitalisées, grabataires ou détenues). La campagne promotionnelle Lire en fête, initiée par le ministère de la Culture, encourage des actions spécifiques vers les écoles, les hôpitaux, les prisons - donnant ainsi raison à ceux qui déplorent que le livre et la bibliothèque soient présentés comme une consolation pour les pauvres ou les malheureux.

Depuis les années 1980, les politiques d'extension de la lecture encouragées par l'État s'adressent prioritairement aux personnes éloignées des bibliothèques et de la lecture - avec les résultats mitigés que l'on ima-

356. Martin Lowell. Library Response to Urban Change: A Study of the Chicago Public Library, Chicago, ALA, 1969, p. XIII ; ALA, "A Mission Statement for Public Libraries", American Libraries, December 1972.

357. Martin Lowell, ibid., p. 20-21. 
gine. La lecture, à partir des années 1990, est progressivement vue comme une activité relevant certes du secteur culturel mais aussi social, voire urbain. Les bibliothèques, enrôlées dans cette extension du domaine, deviennent des acteurs de la lutte contre l'échec scolaire, contre l'illettrisme, contre le délitement du lien social : crise scolaire, crise sociale, crise urbaine changent la donne. Ce glissement n'est pas unanimement approuvé. L'ethnologue Michèle Petit proteste que l'action sociale, dans « les espaces de relégation » que sont les quartiers dits sensibles, porte le risque que les bibliothécaires soient " réduits à animer des ghettos ${ }^{358}$ ». Tandis que des bibliothécaires cherchent un difficile équilibre : " On attend de la bibliothèque qu'elle porte à bout de bras des populations en difficulté socio-économique. On fait de la construction d'une annexe la clef de la réhabilitation d'un quartier sensible. Bref, on place soudain en elle bien des espoirs qu'elle n'est pas toujours à même de combler. À trop miser sur le pouvoir social des bibliothèques, on mélange les genres et on perd leur raison d'être, à savoir constituer et faire vivre des collections. D'où un certain ras-le-bol des bibliothécaires, las de se voir investis de missions relevant plus souvent d'une compétence de travailleur social [...]. Là encore, l'équilibre est difficile à trouver pour la bibliothèque, qui ne peut assumer le rôle de creuset magique de l'intégration mais qui ne peut non plus ignorer l'urgence sociale et se retirer du monde : elle est une bibliothèque dans un territoire. ${ }^{359}$ »

Pourtant, la question des communautés change aujourd'hui, aussi bien en France qu'aux États-Unis. En France, l'expression communautaire est de plus en plus organisée (avec les créations récentes du Conseil français du culte musulman ou du Conseil représentatif des associations noires), la télévision s'empare de la question des " minorités visibles », la discrimination positive est à l'œuvre dans les zones d'éducation prioritaire (même si cette politique est affichée comme territoriale), le gouvernement nomme un " préfet musulman », etc. "L'espace public est ethnicisé », constate Dominique Schnapper ${ }^{360}$. Inversement, aux États-Unis, la politique d'Affirmative Action est aujourd'hui âprement discutée et certains intellectuels considèrent que les communautés sont devenues des territoires balkanisés, qui s'ignorent ou se méprisent les uns les autres et

358. Michèle Petit, Chantal Balley, Raymonde Ladefroux. De la bibliothèque au droit de cité : parcours de jeunes. BPI, 1997, p. 343.

359. Sylvie Fayet, Bruno Van Dooren. « Bibliothèques dans leur territoire », Les bibliothèques en France, 1991-1997, sous la dir. de Dominique Arot. Éditions du Cercle de la Librairie, 1998, p. 74. 360. Dominique Schnapper. La démocratie providentielle, op. cit., p. 209. 
qui portent en germe la mort du « peuple américain ». Christopher Lasch y voit maints effets pervers, de la dégradation continue de la situation des ghettos noirs au « naufrage du système scolaire américain » : "L'effort pour le recrutement d'étudiants issus de groupes économiquement défavorisés, en particulier les communautés noire et hispanique, a occulté une évolution plus importante, la quasi-monopolisation des collèges universitaires et universités les plus célèbres, aussi bien publics que privés, par la grande bourgeoisie [...] Tandis que le débat fait rage entre libéraux et conservateurs sur la révision de programmes accusés d'être euro-centriques, sur les politiques conçues pour promouvoir la diversité et la sensibilité raciales et sur les implications théoriques du post-structuralisme, le problème fondamental passe inaperçu : l'abandon de la mission historique de l'enseignement américain qui était la démocratisation de la culture humaniste. ${ }^{361}$ »

Si le débat est loin d'être clos et que toute conclusion serait aujourd'hui hâtive, on constate cependant des rapprochements sur cette question dans les deux pays. Mais l'écart reste important sur la définition même des minorités à desservir. Aux États-Unis, et c'est là une grande différence qui excède l'histoire politique, c'est la communauté dans toutes ses composantes qui est analysée. Puisqu'elle a besoin du soutien public, la Public Library doit desservir les majorités comme les minorités. La mère de famille, l'écolier, l'étudiant, le jardinier amateur tout comme les Noirs ou les derniers immigrants chinois sont des cibles prioritaires. Cette différence de point de vue a, on l'a vu, une conséquence essentielle sur l'approche des bibliothécaires : pragmatique aux États-Unis (où l'on se préoccupe du public tel qu'il est) et téléologique en France (où c'est le non-usager qui est la cible principale - le public comme on voudrait qu'il soit).

\section{LES COLLECTIONS}

Les bibliothèques ne possèdent pas les mêmes fonds des deux côtés de l'Atlantique. Au-delà de l'évidence, ce constat alimente plusieurs débats, notamment la place du patrimoine, la question de l'offre et de la demande et celle de la censure. Dans ce domaine aussi, les artefacts que sont les bibliothèques se sont développés différemment. 


\section{Le patrimoine}

Point de saisies révolutionnaires aux États-Unis, car point d'aristocratie, d'émigrés ni de congrégations religieuses séculaires. Au demeurant, sur ce point, les bibliothèques françaises diffèrent aussi bien des bibliothèques américaines que des britanniques ou des allemandes. La mise « sous la main de la Nation » de millions de documents (dont environ 4 millions, estime-t-on, se trouvent aujourd'hui dans quelque 300 bibliothèques municipales) a dessiné un profil particulier aux bibliothèques municipales en France.

Le poids du passé se voit là, physiquement, dans ces collections, ces magasins, ces interminables rangées de reliures. C'est là aussi, à lire George Steiner, que l'on mesure l'écart entre l'Europe et l'Amérique : l'Europe, dit-il, vit dans la "souveraineté du souvenir », dans la définition qu'elle se donne d'elle-même " comme d'un lieu de mémoire ». "Quand Paul Celan entre dans la Seine pour se suicider, il choisit l'endroit précis que célèbre Apollinaire dans sa grande ballade, et cet endroit est situé sous les fenêtres de la chambre où Tsvetaïeva a passé sa dernière nuit avant de retourner vers la désolation et la mort en Union soviétique. Un Européen lettré est captif dans la toile d'araignée d'un in memoriam à la fois lumineux et suffocant. C'est ce tissage, précisément, que l'Amérique du Nord récuse. Son idéologie est faite de soleil levant et d'avenir. ${ }^{362}$ »

Les bibliothèques municipales, à leur place, sont elles aussi captives du temps passé, de l'empilement, de la thésaurisation. Mais au-delà du poids de ces collections, souvent considérées comme un handicap, c'est l'idée même de conservation, de la nécessité, de la responsabilité de conserver, qui joue un rôle dans le modèle de bibliothèque. La mise à jour, le « désherbage " sont des impératifs récents. Les «nouveautés » y entrent lentement. L'actualité est réputée y avoir peu de place. C'est tout un rapport au temps qui est modelé par cet héritage.

Le temps des villes : la médiathèque de Limoges, conçue par Pierre Riboulet, annexe l'ancien hôpital de la ville, est dessinée en léger décalage avec l'axe de l'ancienne ville romaine et donne à voir les mosaïques retrouvées lors du chantier. Le projet s'inscrit dans l'épaisseur du temps et « exprime le rattachement à la lignée lointaine ${ }^{363}$ ».

362. George Steiner. Une certaine idée de l'Europe, op. cit., p. 35.

363. Pierre Riboulet, cité dans Anne-Marie Bertrand, Anne Kupiec, Ouvrages et volumes : architecture et bibliothèques. Éditions du Cercle de la Librairie, 1997, p. 169. 
Le temps des morts : "Comme le musée avec lequel elle a des origines communes, la bibliothèque est lieu de mémoire. Elle est don des mots et don des morts. Lieu de conservation des écrits, la bibliothèque doit nouer une relation particulière entre le passé et le présent. ${ }^{364}$ »

Le temps des bibliothèques : " On ferme. On a fermé. Les salles dépeuplées vont être vidées d'un mobilier désormais inutile. Silence. Chaque classeur garde la mémoire des regards qui s'y sont posés, chaque table porte l'empreinte de milliers de mains [...]. Ça sent le papier et la poussière, le vieux bois et le renfermé. C'est un mélange capiteux où domine l'odeur des livres, à la fois suffocante et suave. C'est l'haleine parfumée de l'éternité. ${ }^{365}$ »

Il faudrait se garder de dresser, symétriquement, un portrait stéréotypé des bibliothèques américaines, ancrées dans le présent, tournées vers l'avenir. On voit bien pourtant que le rapport au passé de cette jeune nation est différent, à la fois plus lointain et moins diffus. Comme s'il y avait « des maisons pour ça ». C'est ce que le romancier Don DeLillo laisse entendre : «Et j'aimais la façon dont l'histoire, ici, ne se déchaînait pas. On séparait l'histoire visible. On la mettait en cage, on lui octroyait des fondations et du bronze, on l'enfermait soigneusement dans des musées, des places monumentales et des parcs commémoratifs. Le reste était géographie, espace et lumière, ombre et chaleur immobile indescriptible. ${ }^{366}$ » George Steiner, d'une certaine façon, dit la même chose en soulignant la propension américaine à thésauriser : « Les bibliothèques, les universités, les archives, les musées et les laboratoires de recherche de l'Amérique sont devenus les centres enregistreurs et le reliquaire de la civilisation. C'est là que l'artiste ou le chercheur européen doit aller chercher les feux mourants d'une culture aimée. ${ }^{367}$ "

Pourtant, le passé n'est pas qu'enfermé : des Halls of Fame aux musées d'histoire, des westerns aux champs de bataille de la Guerre de Sécession, du Mount Vernon au cimetière d'Arlington et jusqu'aux Archives nationales, où la Déclaration d'Indépendance et la Constitution sont exhibées et vénérées telles des reliques, le passé est là aussi, bien présent. Cette nation d’immigrants a besoin d'une histoire partagée (« d'éléments dont

364. Martine Poulain. « Bibliothèque ». Dictionnaire encyclopédique du livre, sous la dir. de Pascal Fouché, Daniel Péchoin et Philippe Schuwer. Éditions du Cercle de la Librairie, tome 1, 2002. 365. Claude Bourgeyx. Changement d'adresse. William Blake \& $C^{\circ}, 1990$ (album-souvenir de la Bibliothèque Mably, à Bordeaux, aujourd’hui remplacée par la centrale de Mériadeck). 
on se souvient aussi bien que d'éléments qu'on oublie ", écrit Benedict Anderson), la ruée vers l'or, le naufrage du Titanic, « Il faut sauver le soldat Ryan », l'assassinat de Kennedy...

Rien d'étonnant, donc, à ce que les Public Libraries comprennent souvent des fonds d'histoire locale, pour les plus petites, des fonds patrimoniaux pour les plus grandes, même si ce sont les bibliothèques des universités qui sont les plus riches en ce domaine (contrairement à la France). À Seattle, Bill Gates a généreusement financé la construction de la nouvelle Public Library (vingt millions de dollars) et lui a confié le « codex» de Léonard de Vinci qu'il a acheté récemment.

Mais, enfin, le patrimoine n'est pas une part de l'identité de ces Public Libraries, qui sont au contraire félicitées, par les théoriciens français de la bibliothèque publique, d'être exemptées de ce poids. Ainsi, Jean Hassenforder, à propos des bibliothèques publiques du XIX siècle : "En Grande-Bretagne et aux États-Unis, les bibliothèques municipales sont, au départ, des organismes nouveaux qui peuvent innover. Elles allient une double fonction : documentation et diffusion du livre auprès du grand public, et les services rendus se diversifient progressivement en rapport avec les besoins. Les méthodes sont commandées par le souci de satisfaire l'usager et de lui offrir la documentation la plus actuelle. En France, au contraire, les vieilles bibliothèques municipales se révèlent un poids mort. Elles se consacrent à la conservation des fonds anciens et constituent le domaine des érudits. ${ }^{368}$ "

Les livres vieillissent mal, écrit Eugène Morel. " Le livre n'est pas comme le vin, qui gagne en vieillissant. Il est comme un habit qui se démode et qui s'use, il vit et meurt ; utile, porté jusqu'à la corde, sale et fripé, on s'en sépare avec regret ; mal fait ou luxueux, gardé précieusement, il ne sort de l'armoire où il se mange aux vers qu'aux grandes cérémonies, et son air suranné couvre de ridicule celui qui s'en pare! Portez vos livres neufs! Usez vos livres. Cela n'empêchera pas d'aller aux Invalides ou à la Bibliothèque nationale, voir de vieux habits et de vieux livres sous verre. Je garde pieusement un gilet de mon bisaïeul. Il est brodé de fleurs roses et très bien conservé. Mais ce n'est pas celui que je porte ordinairement. ${ }^{369}$ »

En France, la mise en cause du poids du patrimoine (comme facteur d'inertie et comme image négative) est devenue un exercice de style.

368. Jean Hassenforder. Développement comparé des bibliothèques publiques en France, en GrandeBretagne et aux États-Unis, op. cit., p. 197.

369. Eugène Morel. Bibliothèques. Mercure de France, 1909, p. 9. 
Dès 1972, le rapport La lecture publique en France reprend à son compte l'opinion fréquemment émise : "Les bibliothèques municipales ont été victimes, autant que bénéficiaires, du prestigieux héritage qu'il leur incombait de conserver. » Alors même que l'institutionnalisation du patrimoine écrit est en marche (l'Année du patrimoine, le rapport Desgraves sur le patrimoine, le début des aides de l'État à l'entretien du patrimoine écrit, la création du Colloque du patrimoine écrit, etc.), le directeur du livre, Jean Gattégno, croit nécessaire de s'en expliquer : «Si en 1980, la Direction du livre et de la lecture avait dit qu'elle voulait faire un grand effort patrimonial, cela aurait été interprété par la partie la plus dynamique des bibliothécaires de lecture publique comme une crispation sur le passé, sinon comme un retour en arrière [...]. Je crois qu'on ne pouvait valablement et qu'on ne peut valablement vouloir pousser la recherche patrimoniale et la mise en valeur du patrimoine des bibliothèques publiques que si on a antérieurement, et non pas parallèlement, impulsé un dynamisme suffisamment grand à la lecture publique en tant que telle. ${ }^{370}$ »

Presque dix ans plus tard, les mêmes discours, les mêmes doutes sont toujours là : " L'importance des collections patrimoniales issues des confiscations révolutionnaires - collections dont il ne s'agit pas du tout ici de nier l'intérêt - a longtemps freiné le développement de la lecture publique dans un certain nombre de bibliothèques. ${ }^{371}$ » Et l'on pourrait multiplier les exemples.

Jusqu'à aujourd'hui, même si le discours est moins audible, le patrimoine est souvent vécu par les bibliothécaires français comme un poids qui obère le développement des bibliothèques municipales. Un poids regrettable que n'ont pas à porter les Public Libraries...

\section{L'offre et la demande}

Quelle ligne directrice doit guider la constitution des collections, c'est-àdire, pour faire vite, le choix des livres à acheter ? Les bibliothécaires sont en désaccord entre eux en ce domaine, d'un côté comme de l'autre de l'Atlantique.

Ici s'affrontent d'un côté la légitimité des bibliothèques, leurs missions, objectifs, fondements : la formation, le partage du savoir, la partici- 
pation à la vie démocratique, l'information, la documentation. De l'autre côté, la légitimité des bibliothèques (une autre légitimité), la demande des usagers, les besoins de la population, la satisfaction du public - satisfaction sur laquelle s'appuie le soutien public. Mais si cette tension entre deux légitimités existe dans les deux pays, elle est gérée différemment.

Les racines des Public Libraries, au XIX ${ }^{e}$ siècle, font d'elles une institution éducative, chargée de proposer les documents et informations dont les citoyens ont besoin pour se former tout au long de leur vie et dont ils ont besoin pour exercer de façon éclairée leur rôle de citoyen. Certains bibliothécaires, certaines bibliothèques continuent aujourd'hui à exercer ce rôle. D'autres voient dans ce rôle un mensonge destiné à perpétuer le statu quo et appellent à une implication sociale, en particulier auprès des minorités ethniques. D’autres, enfin, arguant de la nécessité pour les Public Libraries d'obtenir et conserver le soutien public, avancent que le rôle des bibliothèques est, prioritairement, de donner aux usagers ce qu'ils souhaitent : Give them what they want est leur credo. Douglas Raber a défini ces trois types de bibliothécaires comme des conservateurs, des activistes sociaux et des populistes ${ }^{372}$. On pourrait sans doute, par ordre d'entrée en scène, y ajouter une quatrième famille, les bibliothécaires technicistes qui pensent que les nouvelles technologies de l'information sont la réponse universelle aux attentes de la population.

Si les quatre familles idéologiques sont présentes aujourd'hui dans le groupe professionnel des bibliothécaires américains, le contexte budgétaire (en baisse) et social (le respect de la diversité) semblent bien les faire pencher vers la réponse à la demande (« Donnez-leur ce qu'ils veulent ! »). De bons budgets d'acquisition permettent d'avoir des collections diversifiées, éclectiques. Mais une demande pressante et pressée augmente le poids pris par la fiction, le respect des modes (notamment la fantasy) et des achats en multiples exemplaires (à l'été 2005, 30 à 50 exemplaires pour Harry Potter and the Halfblood Prince dans les bibliothèques moyennes, 1055 exemplaires à la New York Public Library). C'est ainsi, analysent Françoise Gaudet et Claudine Lieber, que la concurrence d'Internet est combattue : « Don Napoli, directeur de la Saint Joseph County Public Library (South Bend, Indiana) annonce lui aussi de bons chiffres de prêts, de même que Gary Strong, à la Queens Borough Public Library (New York) - bibliothèque qui s'enorgueillit d'être la plus active des États- 
Unis avec 17 millions de prêts par an. Tous deux expliquent que leur politique consiste à donner aux usagers ce qu'ils veulent et à mettre l'accent sur les collections populaires, les best-sellers et l'audiovisuel. ${ }^{373}$ »

En France, le retard dans l'offre documentaire a été pour partie résorbé : les bibliothèques municipales possédaient 34 millions d'imprimés en 1974, 103 millions en 2002. Cette augmentation s'est faite par un élargissement du périmètre culturel considéré comme légitime : les bandes dessinées, les romans policiers, la science-fiction, les romans sentimentaux (dans une moindre mesure) sont entrés massivement dans les collections. En dehors de la fiction, cependant, la diversification en genres d'ouvrages est moins nette : la vulgarisation scientifique, les ouvrages pratiques, les livres d'actualité (en particulier les mémoires ou récits des vedettes médiatiques) sont toujours peu présents. Ce simple constat montre que les bibliothécaires sont sensibles à la demande - mais pas trop ! Le discours dominant est aujourd'hui contradictoire, brouillé, les appelant en même temps à une exigence de qualité (donc à une politique d'offre) et à une nécessité d'attractivité (donc à une réponse à la demande).

Naviguant entre une espèce d'entre-deux, les collections montrent ainsi ce que Dominique Arot appelle un " goût moyen », plutôt consensuel et sans aspérités, où la littérature est encore valorisée par rapport aux savoirs pratiques. Quand on leur en parle, les bibliothécaires américains qui les connaissent qualifient les collections des bibliothèques françaises d'élitistes.

On peut avancer que, si une plus grande ouverture s'est faite, les bibliothécaires français continuent, en effet, à penser que tous les documents n'ont pas leur place dans les collections des bibliothèques. Cette conviction est à la fois partagée et implicite. Partagée parce qu'elle repose sur les représentations qu'ont les bibliothécaires de leur rôle - même si ces représentations évoluent. Ainsi, Dominique Tabah : « À céder aux modes, aux goûts et aux pressions du jour, les bibliothèques risquent en fait de ne plus remplir leur mission culturelle et sociale, de ne plus jouer leur rôle de résistance et de n'être plus que des espaces de culture consensuelle, abandonnant toute ambition de promouvoir les œuvres, les auteurs et les éditeurs qui prennent des risques. ${ }^{374}$ " Conviction partagée car les

373. Françoise Gaudet, Claudine Lieber. «L'Amérique à votre porte : nouveaux usages, nouveaux services, nouveaux concepts de bâtiments... un avant-goût de notre avenir ? ». Bulletin des bibliothèques de France, t. 47, n 6, 2002.

374. Dominique Tabah. "Le rôle social et culturel des bibliothèques vu de Bobigny ». Bulletin des bibliothèques de France, t. 42, $\mathrm{n}^{\circ}$ 1, 1997. 
collections de la bibliothèque présentent une image du monde - une organisation des savoirs, un état de la création, une mémoire du territoire. Image nécessairement ambitieuse car guidée par le projet de partage des savoirs. Image, donc, nécessairement excluante.

Mais, si elle est partagée, cette conviction est également implicite, parce que le sujet est peu débattu. En matière de collections, les procédures et les techniques semblent intéresser davantage les bibliothécaires que des discussions sur les niveaux de lecture, les livres en français facile ou les livres pour des lecteurs qui ne lisent pas Télérama. À l'exception, sans doute, des bibliothécaires pour enfants qui, eux (elles ?), continuent à explorer la production éditoriale pour la jeunesse et à élaborer des critères de sélection. Sélection? On a l'impression que ce mot est inconnu des bibliothécaires américains.

Les lecteurs français, qui utilisent ou ont utilisé les collections des bibliothèques municipales, ont, eux, le sentiment qu'elles leur proposent une offre à la richesse inégale : une enquête menée en 1995 montrait que si $90 \%$ des lecteurs sont satisfaits des fonds de littérature classique ou $88 \%$ des romans contemporains, le taux de satisfaction tombe à $57 \%$ pour les livres scientifiques et techniques, à $50 \%$ pour les sciences humaines et sociales ou à $31 \%$ pour les livres pratiques ${ }^{375}$. L'héritage de la bibliothèque savante est bien toujours là !

Paradoxe : si les bibliothécaires ont su, par le biais des médiathèques, offrir de nouveaux services, diversifier l'offre, moderniser l'image de l'établissement, les collections sont, elles, restées largement homogènes, comme si l'augmentation et la diversification des publics n'appelaient pas une augmentation ni une diversification de l'offre documentaire. Et dans les deux sens, comme le souhaitait Lowell Martin : des livres plus faciles et des livres plus exigeants (des films plus faciles et des films plus exigeants, des documents plus faciles et des documents plus exigeants...).

La réflexion sur les collections est, évidemment, compliquée par les nouveaux modes d'accès à la connaissance et au savoir (Internet, les moteurs de recherche, les blogs) qui mettent à mal le paradigme encyclopédique qui sous-tend le projet des bibliothèques. L'offre des bibliothèques (en France comme aux États-Unis) n'est plus un espace clos, une sphère autosuffisante - si tant est qu'elle l'ait jamais été ! Le débat, cependant, reste hésitant, peu actif, comme si l'on craignait, une fois encore, « de se cris- 
per sur les sempiternelles oppositions entre l'universalisme prescripteur à la française (l'idéal d'une collection parfaite), le consumérisme individualiste (faire du chiffre avec "ce qui sort le plus") et le communautarisme à l'anglo-saxonne (la juxtaposition des fonds ethniques). ${ }^{376}$ »

\section{La censure}

En 1996, environ 450 cas de censure ont été signalés à l'ALA - la très grande majorité dans les bibliothèques scolaires ${ }^{377}$. La liste des livres censurés ou menacés est très longue, analyse Jack Kessler, « et l'éventail des auteurs des "Cent livres les plus souvent contestés" ${ }^{378}$ est d'une étonnante hétérogénéité, même si dans une société aussi diversifiée que celle qui peuple aujourd'hui les États-Unis chacun a forcément des tas de raisons de désapprouver des tas de choses. Figurer sur la liste des "proscrits" devient une marque de notoriété dont beaucoup d'écrivains sont fiers. Ici ou là sur le territoire des États-Unis, sous un prétexte ou sous un autre, des bibliothèques ont ces derniers temps retiré de leurs rayonnages des œuvres de John Steinbeck, Judy Blume, J. D. Salinger, Alice Walker, Madonna, Harper Lee, Aldous Huxley, Roald Dahl, Stephen King, Isabel Allende, Kurt Vonnegut, William Golding, Toni Morrison, ainsi que les déjà cités Mark Twain et J. K. Rowling. ${ }^{379}$ » Mais, ajoute Jack Kessler, "ce sont des exceptions » : si 6364 tentatives de censure ont été signalées à l'ALA entre 1990 et 2000, chacune (ou presque) a été combattue avec ardeur ${ }^{380}$.

Tout a sa place dans les Public Libraries, soutient l'ALA. Aucun texte, aucune opinion n'y sont déplacés puisqu'ils aident le citoyen à se forger sa propre opinion - on se souvient du premier nom du Comité pour la liberté d'information de l'ALA, " comité de la liberté d'information pour protéger les droits des usagers des bibliothèques à la libre investigation ». Les opinions minoritaires, provocatrices, scandaleuses ont leur place dans les Public Libraries. L'effet pervers de cette acceptation universelle

376. Patrick Bazin. «Plus proches des lointains ». Bulletin des bibliothèques de France, t. 49, $\mathrm{n}^{\circ} 2$, 2004.

377. Caroline Rives. «La censure aux États-Unis ». Bulletin d’informations de l’ABF, $\mathrm{n}^{\circ}$ 175, 1997.

378. "The 100 Most-Challenged Books List of 1990-2000",

< http://www.ala.org/ala/oif/bannedbooksweek/bbwlinks/100mostfrequently.htm >

379. Jack Kessler, " Religion et bibliothèques aux États-Unis », Bulletin des bibliothèques de France, t. $48, n^{\circ} 6,2003$.

380. L'ALA, au bas de la liste des 100 livres les plus contestés, indique aussi que sa collation des incidents n'est pas exhaustive et que « la recherche suggère que pour chaque incident signalé, il y en a quatre ou cinq qui ne le sont pas ». 
est que le rôle du bibliothécaire n'est plus de choisir : il est d'acquiescer. " Effet pervers » aux yeux des bibliothécaires français mais aussi américains.

Dans un éditorial à propos de la présence de textes révisionnistes dans les Public Libraries, John Berry, rédacteur en chef du Library Journal, est pour le moins circonspect : "Des réponses faciles et des récitations à genoux du Library Bill of Rights ne résolvent pas le problème des bibliothécaires qui font face aux pressions de groupes extrémistes. Nier qu'il y a des livres si inappropriés qu'ils doivent être rejetés est aussi bête que nier l'holocauste. » Et, si tout livre, tout document a sa place dans la bibliothèque comme le soutient l'ALA, cela soulève une autre question : «En d'autres termes, une collection ne peut pas être sélectionnée du tout. Les bibliothécaires doivent simplement acheter ce que n'importe quelle personne ou n'importe quel groupe demande - des documents sur le traitement du cancer par les noyaux d'abricots, des régimes sans aucune graisse, des atlas où la terre est plate, et les travaux des menteurs qui disent que l'holocauste n'a pas existé. ${ }^{381}$ » Heureusement, dit-il, les bibliothécaires n'appliquent pas les principes de l'ALA aveuglément - ce qui déplace le problème vers celui de l'écart entre principes et pratiques.

En France, le débat est récurrent : doit-on tout accepter ? Peut-on, at-on le droit de sélectionner, choisir, censurer? Si les bibliothécaires sont opposés aux interventions extérieures (et spécialement aux interventions des élus) sur leurs choix d'achat, ils ont aussi des doutes sur leur propre pouvoir en ce domaine. Bien entendu, l'autocensure est la réponse la plus fréquente à ces doutes : s'il risque d'y avoir problème (un livre discuté, une position discutable), il est prudent de ne pas l'acheter. Position évidemment non explicitée ni rendue publique. Mais la question du choix fait, de temps en temps, l'objet d'un débat public, comme à l'hiver 2003 : faut-il, peut-on acheter Mein Kampf, que faire des livres de Maurras ou Gobineau qui ont été achetés avant guerre et sont toujours dans le fonds?

Les réponses sont souvent tirées vers le registre technique : « La définition d'une politique documentaire, la mise en œuvre d'une politique d'acquisition précise et clairement établie me semble abolir le sens même d'une censure quelconque. Dès lors que l'on a défini les objectifs de développement des collections, l'idée même de censure ne saurait avoir la moindre place. En outre, la pratique affirmée et revendiquée (assumée ?) d'une censure peut paraître étrange en termes purement techniques : 
quelle valeur professionnelle cela saurait avoir ? Pouvons-nous envisager des formations à la censure en bibliothèque ? ${ }^{382}$ »

Mais l'on observe aussi des bibliothécaires qui assument clairement leur refus d'acheter certains titres : « Il y a la réflexion au chaud, déontologique, professionnelle et confortable..., et cela est bien. Et puis... la réflexion à chaud, déontologique tout autant et professionnelle itou, et pas vraiment confortable, quand ça urge beaucoup, et qu'on doit riposter, et vite, face à des élus FN qu'on a sur le dos, et qu'on comprend très vite, plus vite qu'ailleurs, ce que veut dire le mot “démocratie”. Et puis il y a toutes les petites entreprises de saloperie chic (Renaud Camus et Houellebecq et consorts...) et qui passent et qui font jaser et qui occupent un temps les gazettes, et qui font efficacement leur sale petit boulot... ${ }^{383}$ » Et des bibliothécaires, au contraire, qui assument clairement leur décision d'acheter les titres qui suscitent le débat (ici, Charles Maurras, De la politique naturelle au nationalisme intégral) : "Maurras c'est aussi de belles pages sur la Provence, Rebatet c'est l'indigeste Les décombres et le merveilleux Les deux étendards, doit-on interdire l'auteur, les deux œuvres, une seule, aucune ? Mon humble avis est qu'il n'y a rien à gagner à censurer (sauf si la loi l'oblige), à avoir peur de lire et qu'il est toujours préférable de laisser aux lecteurs (à moins de les prendre pour des imbéciles) la possibilité de lire et de se forger une opinion. De plus ce livre de Maurras peut intéresser certes ses admirateurs mais aussi ses opposants, des historiens et des personnes qui en ont entendu parler et qui ont envie de le connaître et de le lire. ${ }^{384}$ » Les bibliothécaires français, on le voit, sont loin d'être unanimes sur ce sujet.

Les positions sont également différentes de celles d'Outre-Atlantique quant à l'accès libre à Internet. L'accès à Internet dans les bibliothèques municipales françaises est encadré, filtré, bridé. La plupart du temps, la messagerie, les jeux en ligne, les chats sont interdits (et techniquement rendus difficiles). La chasse aux sites pornographiques, nazis, racistes ou pédophiles est ouverte (et sans fin...). En somme, accès libre mais pas trop. Ce qui marque un nouvel écart d'avec les Public Libraries, plus enclines à compter sur le contrôle social (les usagers se surveillant les uns les autres car consultant Internet en présence les uns des autres) que sur un dispositif institutionnel. L'exigence du gouvernement fédéral de filtrer Internet, dans le cadre du CIPA, a provoqué, on l'a vu, un combat frontal. 


\section{L'IMAGE DE LA BIBLIOTHĖQUE}

Si la place des bibliothèques dans la société est si différente d'un pays à l'autre, on doit pouvoir le vérifier par deux biais : d'une part, par la présence des bibliothèques dans l'imaginaire social, dans les pratiques quotidiennes, dans les stéréotypes de la vie quotidienne ; d'autre part, par le poids de l'absence (les réactions sociales à l'absence de bibliothèque). Tentons cet exercice.

\section{La bibliothèque dans le paysage social}

Quel lecteur français n'a été frappé par la présence fréquente, banale, des Public Libraries dans les œuvres de fiction, qu'elles soient des films ou des livres? «Quels liens peut-on faire entre des blockbusters américains tels que The day after tomorrow, Seven, la plupart des romans policiers de l'Écossais Ian Rankin, Lumière morte, l'un des derniers livres de Michael Connelly, Charlie de Stephen King ou encore Ne le dis à personne, le best-seller d'Harlan Coben ? 1. Ce sont des créations ou productions contemporaines à succès (populaires pourrait-on dire). 2. Elles sont toutes anglo-saxonnes. 3. Les bibliothèques publiques, à chaque fois, y sont présentes et dans certains cas y jouent un rôle important. On trouvera difficilement en France, ou dans les pays de l'Europe du Sud, un équivalent de cette présence régulière des bibliothèques dans les œuvres de fiction grand public. ${ }^{385}$ »

Bien plus, on peut voir dans les œuvres l'image et l'usage que Monsieur Toutlemonde fait des Public Libraries. Enfant, adolescent, on fréquente la bibliothèque pour ses devoirs, pour trouver des informations (Back to the Future), pour retrouver les copains (Elephant) ou pour « découvrir le monde ${ }^{386}$.

Adulte, on y va parce que c'est un lieu accueillant : " Puis elle se rendit à la bibliothèque municipale de la Quarante-Deuxième Rue. Elle n'y manquait jamais lorsqu'elle se trouvait à New York. Elle allait dans les musées, dans les galeries, au concert, au cinéma voir les films qui ne passeraient jamais dans l'effroyable salle de ce bled d'Athena, et, pour finir, quelles qu'aient pu être les raisons qui l'amenaient à New York, elle venait

385. Christophe Evans. «Quels développements pour la recherche sur les publics ? ». Bulletin des bibliothèques de France, t. 50, n², 2005.

386. « Nous faire découvir le monde, surtout par la lecture : telle était la priorité de ma mère. Elle m'emmenait à la bibliothèque chaque semaine et je me plongeais avec ravissement dans les livres de la section enfantine» (Hillary Rodham Clinton, Mon histoire, Fayard, 2003, p. 24). 
passer une heure ou deux dans la grande salle de lecture, pour lire le livre qu'elle avait apporté avec elle [...]. De sorte que la bibliothèque a toujours eu à ses yeux cette aura romanesque. ${ }^{387}$ »

On y va parce qu'on y trouve les œuvres de son fils : «Il m'a un jour écrit, quand j'habitais encore Paris, pour me raconter qu'il était allé à la bibliothèque publique lire certains de mes poèmes récemment parus dans la revue Poetry. Je me l'imaginais, dans une grande salle déserte, tôt le matin avant d'aller travailler, assis à l'une de ces longues tables, son pardessus sur le dos, courbé sur des mots qui devaient lui sembler incompréhensibles. ${ }^{388}$ »

On y va pour faire des recherches : « À la bibliothèque municipale de Newark, je lus de près les pages sportives du Newark News (disparu en 1972) sur microfilms pour y chercher les comptes rendus et les résultats des matches où le Suédois avait brillé pour le lycée de Weequahic (sur le point de fermer) et pour la faculté d'Upsala (fermée en 1995). ${ }^{389}$ "

On y va parce qu'on y est à l'aise : " J'y étais revenu après l'achèvement des travaux de restauration, pour la première fois depuis mon enfance. Et je n'avais pas cessé d'y retourner. Je n'avais jamais été un grand lecteur. J'étais incapable de tenir en place assez longtemps. C'était pour le lieu que je le faisais. Ça me rappelait le Los Angeles que j'avais gardé en mémoire. Celui où je me sentais à l'aise. J'emportais mon sandwich dans les salles de lecture ou les patios du dernier étage et y lisais mes dossiers d'enquête ou prenais des notes. Je connaissais tous les gardiens et tous les bibliothécaires. J'avais même une carte, bien que je ne sorte jamais rien. ${ }^{390}$ »

Avoir une carte de bibliothèque est plus courant qu'avoir une pièce d'identité et permet d'identifier un cadavre : «Au début, on n'avait qu'une idée très vague de son identité. On lui avait vidé les poches, on avait examiné le contenu de son portefeuille, et on n'avait trouvé ni permis de conduire, ni passeport, aucun papier d'identité de quelque espèce que ce fût. La seule indication d'un nom se trouvait sur une carte de membre de la branche nord de la bibliothèque publique de Chicago. ${ }^{391}$ "

Quand le héros gagne à la loterie, il fait un don à sa Public Library ${ }^{392}$. Il lui arrive d'avoir des parents bibliothécaires (Hours, Petite visite aux 
cannibales). Il veut faire plaisir à la bibliothécaire (Charlie Brown). Bref, la bibliothèque existe dans le paysage quotidien.

On serait évidemment bien en peine de dresser un florilège similaire dans la fiction française. Le film de Rohmer, L'arbre, le maire et la médiathèque, était une pure fiction et non l'insertion d'une réalité dans une fiction. Ici ou là, cependant, on trouve des traces. Quelquefois brèves : «Ce septembre-là, 1948, Mathilde consulte l'encyclopédie Larousse à la bibliothèque publique d'Hossegor. ${ }^{393}$ "Quelquefois plus détaillées : « J'attendais les mardis soirs, les mercredis et les week-ends - les Pepito devant le film de vingt heures trente, la poterie, le piano, la danse, la section jeunesse de la bibliothèque de la rue Hermel, les livres sur lesquels était écrit "N'abîmez pas les livres, ils sont notre bien commun". ${ }^{394}$ "

Cette présence beaucoup moins courante de la bibliothèque, de l'usage de la bibliothèque, reflète évidemment une moindre culture de la bibliothèque. Même si elle est nettement plus fréquentée qu'il y a trente ans, la bibliothèque municipale demeure un endroit spécial, un peu étranger, auquel on n'adhère pas tout à fait. Elle est l'objet d'une " estime lointaine ${ }^{395} »$ : on veut bien croire qu'elle est utile, mais on ne la sent ni nécessaire ni proche.

C'est sans doute pourquoi son absence ne suscite pas (guère) de mobilisation.

\section{L'absence de bibliothèque}

En France, la bibliothèque municipale est une offre trop éloignée de la population pour susciter la mobilisation de ceux qui n'en ont pas - ou à qui on veut l'enlever. Pourtant, la situation semblerait en train d'évoluer. Des habitants de Marseille se sont groupés en collectif ("Médiathèque Rivoire et Carret ») pour que, après l'ouverture de la grande bibliothèque de l'Alcazar, les quartiers Est soient, à leur tour, dotés d'un établissement adapté ${ }^{396}$. La fermeture de petites bibliothèques de quartier, proches de la nouvelle bibliothèque centrale de Toulouse, provoque l'incompréhension et l'indignation. Même indignation à Paris où le projet de fermeture de la

393. Sébastien Japrisot. Un long dimanche de fiançailles. Denoël, 1991.

394. Clémence Boulouque. Mort d'un silence. Gallimard, 2003. Un grand amoureux des bibliothèques, Jacques Roubaud (qui se proclame homo bibliothecus), n'exprime son amour qu'à la Bibliothèque nationale.

395. Anne-Marie Bertrand. «Une estime lointaine : les non-usagers des bibliothèques municipales ». Bulletin des bibliothèques de France, t. 43, $n^{\circ}$ 5, 1998.

396. Livres Hebdo, 3 mars 2006. 
bibliothèque Vaugirard suscite une pétition en ligne (2007) et la création d'un groupe Facebook « Non à la fermeture de la bibliothèque Vaugirard » (2009).

Au plan national, une cinquantaine de villes de plus de 10000 habitants n'ont pas de bibliothèque municipale. Il s'agit surtout de villes de banlieue dont les élus considèrent que la bibliothèque de la ville-centre suffit aux besoins de leurs administrés - c'est la raison qui est avancée par des élus de l'agglomération de Roanne. Beaucoup d'autres villes ont des bibliothèques mais qui sont très petites, archaïques, sans véritable budget d'acquisitions, sans personnel qualifié. En 1990, Evelyne Pisier, alors directeur du livre, écrivait : " La tâche qui reste à accomplir est immense [...]. La réussite des grandes médiathèques, qui focalisent l'attention, ne doit pas dissimuler qu'il y a encore trop d'équipements médiocres, inadaptés, vieillis. ${ }^{397}$ » Même si des progrès ont été faits, le constat est le même aujourd'hui - sans susciter l'indignation citoyenne.

Aux États-Unis, où la population est desservie à l'exception de quelques zones montagneuses ou désertiques (soit 2,8\% de la population), c'est une autre alarme qui est tirée : même si tout le monde est en théorie desservi, l'offre n'est pas si positive. Ainsi, Michael Gorman évoque les lacunes des services des bibliothèques pour « les vieux, les jeunes, les pauvres, les handicapés physiques ou mentaux, les membres des minorités ethniques ou ceux qui habitent à la campagne ". Pour eux, les services des bibliothèques sont moins accessibles que pour ceux qui ne font partie d'aucune de ces catégories ${ }^{398}$. Il y a aujourd'hui, écrit-il, une « fracture bibliothécaire » (library services divide).

Bien pire, écrit un autre analyste, Michael Sullivan, la situation se dégrade avec la fermeture de bibliothèques - il cite un certain nombre d'exemples, dont huit bibliothèques de proximité dans le comté de Multnomah (Oregon) en 1997, cinq des trente-six annexes de quartier de l'Enoch Pratt Library de Baltimore en 2001 ou quarante-trois des quatrevingt-sept bibliothèques du Comté de Los Angeles en 1993. Il ne s'agit pas que de statistiques : «Quand nous parlons de fermeture de bibliothèques de quartier, gardez à l'esprit que ces fermetures signifient souvent la fin des services de bibliothèque pour un quartier, une communauté ou même une ville tout entière. ${ }^{399}$ »

397. Préface à Bibliothèques municipales. Statistiques 1987. Ministère de la Culture, 1990.

398. Michael Gorman. The Enduring Library: Technology, Tradition and the Quest for Balance. ALA, 2003, p. 119.

399. Michael Sullivan. “The Fragile Future of Public Libraries”. Public Libraries, September-October 2003. 
La diminution des budgets publics et la concurrence d'Internet alimentent le discours qui justifie la fermeture des bibliothèques - ou leur affaiblissement ${ }^{400}$. Mais c'est que l'on oublie alors le caractère public (publicness) des Public Libraries dont le premier rôle est de « fournir l'égalité d'accès à l'information et au monde du savoir [...]. Public est le mot qui est la plupart du temps oublié par ceux qui proclament que la Public Library n'a pas d'avenir. ${ }^{401}$ " L'avenir de la bibliothèque est, au contraire, qu'elle est un bien commun (public good). Cette conviction (cette rhétorique ?) est rarement exprimée avec cette force en France. La bibliothèque est supposée être bonne en soi, sans qu'il soit besoin d'argumenter, de plaider, de convaincre - l'advocacy est absente.

On a longtemps cru (et écrit) en France que les Français ne fréquentaient pas les bibliothèques parce qu'il n'y en avait pas. Mais que, pour peu que l'offre soit quantitativement et qualitativement améliorée, le problème serait résolu. Même si les chiffres montrent une progression très nette (un Français sur dix fréquentait une bibliothèque municipale en 1980, un sur quatre ou un sur trois, selon les sources, aujourd'hui), la bibliothèque municipale n'est pas devenue l'élément de base qu'elle est aux États-Unis.

Aujourd'hui, où le discours français sur la pénurie de l'offre ne peut plus être tenu, on évoque volontiers l'absence de " culture de la bibliothèque » pour expliquer cette persistante faiblesse. C'est, en somme, une autre forme de l'explication antérieure : on disait jadis que l'absence de bibliothèque expliquait la faible fréquentation; on dit aujourd'hui que l'absence de familiarité avec la bibliothèque, l'absence d'habitude, en somme l'absence de fréquentation expliquent la faible fréquentation. Après l'analyse naïve, l'analyse tautologique.

À l'issue de ce travail, on peut avancer un autre type d'explication : ce n'est pas l'absence, c'est l'éloignement (politique, culturel, symbolique) qui peut expliquer que la bibliothèque continue à être peu présente. Aujourd'hui, le paradigme bibliothécaire (une offre encyclopédique et cohérente dont les non-usagers sont la cible principale) aurait-il trouvé ses limites?

400. En 2003, un referendum organisé au Mesa County (Colorado) pour financer un nouveau bâtiment pour la bibliothèque n'a pas réuni de majorité. L'une des opposantes expliquait ainsi sa position : « Je crois que les bibliothèques sont en train de devenir des dinosaures avec Internet et tout l'électronique qui est disponible dans la plupart des familles maintenant. Il n'y a qu'une petite proportion de la population qui utilise la bibliothèque. » ("Referenda Roundup, 2003: Many Celebrations, Some Major Disappointments”, < http://www.ala.org >)

401. Susan Goldberg Kent. "American Public Libraries », Books, Bricks \& Bytes: Libraries in the Twenty-First Century. Transaction Publishers, 1999, p. 209-212. 


\section{CONCLUSION}

L'

utilité sociale des bibliothèques publiques, née de leurs fondements politiques, diffère des deux côtés de l'Atlantique. Je sou-

haite rappeler ici brièvement quatre divergences majeures, toutes quatre de nature politique : les bibliothèques et leur rôle éducatif ; les bibliothèques dans la vie démocratique ; le soutien public ; l'advocacy. Mais ces divergences, identifiées dans ce travail, ne recouvrent pas entièrement les différences entre les bibliothèques municipales et les Public Libraries : ces différences relèvent aussi du rapport au savoir et du partage de ce savoir. Enfin, comme conclusion à cette conclusion, je voudrais revenir sur une différence d'essence, celle qui a trait au projet que portent les bibliothèques.

\section{QUATRE DIVERGENCES}

\section{Bibliothèques publiques et formation}

La tradition américaine d'éducation des immigrants, la place de la formation des adultes et l'usage des Public Libraries par un public majoritairement enfantin ou jeune : ces trois éléments ont contribué à ce que l'objectif éducatif y demeure très vivace et très visible, avec les reference libraries (et les reference librarians), l'aide aux devoirs, des salles dévolues au travail silencieux, des cours aux adultes et des collections de documents constituées à cet effet. En France, les sections d'étude ont disparu car elles étaient considérées comme discriminantes ; ni aide aux devoirs ni cours ; pas de bibliothécaires spécialisés dans ce travail ; des étudiants vus comme des ennemis qui détournent à leur profit un établissement qui ne leur est pas destiné.

Les Public Libraries continuent à assumer les missions assignées par leurs fondateurs. En France, le modèle de bibliothèque publique aujourd'hui mis en œuvre semble avoir pour objet (comme un de ses objets) de se démarquer des bibliothèques savantes d'autrefois - et, même, de se démarquer du modèle de bibliothèque publique promu par les modernistes du 
$\mathrm{xx}^{\mathrm{e}}$ siècle, d'Eugène Morel à Michel Bouvy en l'amputant de son volet éducatif. Les Public Libraries sont, sciemment, volontairement, un complément de l'école et une université populaire ; les bibliothèques municipales ont voulu déscolariser la lecture et manifester ostensiblement leur éloignement du monde scolaire ${ }^{402}$.

Les conséquences de cet état de fait français sont fâcheuses pour au moins deux raisons. En projetant les bibliothèques du côté des loisirs, il les prive d'une fréquentation utilitaire de la part d'une population qui en aurait pourtant besoin. En les inscrivant dans une consommation culturelle vue comme du superflu, du " supplément d'âme », il jette le doute sur le bien-fondé des financements publics. C'est l'utilité sociale de la bibliothèque qui justifie sa prise en charge par la communauté - et l'utilité est davantage du côté de la formation que de la « lecture-plaisir » prônée par les bibliothécaires. Les bibliothèques municipales françaises, privées de leur pôle éducatif, boitent.

\section{Les bibliothèques dans la vie démocratique}

"La bibliothèque est un service public nécessaire à l'exercice de la démocratie. Elle doit assurer l'égalité d'accès à la lecture et aux sources documentaires, pour permettre l'indépendance intellectuelle de chaque individu et contribuer au progrès de la société. » (Charte des bibliothèques, article 3)

Les bibliothèques municipales françaises se targuent volontiers de leur rôle dans la formation des citoyens ou dans l'exercice de la vie démocratique. Pourtant, dans le même temps, elles expriment une aversion inquiétante pour la vie politique, au point que certains bibliothécaires suggèrent de supprimer les livres politiques des rayons. Former les citoyens et encourager la vie démocratique c'est, au contraire, informer, donner des outils de connaissance et de compréhension - comme le font les Public Libraries en étant le relais de la vie politique et associative locale, en donnant accès à l'information gouvernementale, en identifiant les décideurs politiques et leur agenda. Là-bas, il s'agit d'une démocratie active,

402. On voudra bien, en me lisant, faire la part de la généralisation obligée et obligatoirement injuste - il est clair que certaines bibliothèques municipales, dont les plus grandes mais pas seulement, continuent à promouvoir leur rôle éducatif. Mais l'attitude majoritaire et les discours autorisés, la doxa, considèrent que c'est un dévoiement des missions et un handicap pour leur image. Au demeurant, les jeunes scolarisés (collégiens, lycéens, étudiants) tordent eux-mêmes le bâton dans l'autre sens en demandant à la bibliothèque ce qu'elle ne souhaite pas leur donner un accompagnement de leur parcours scolaire. 
appuyée sur ses nombreux groupes, comités et autres associations ; ici, la vie démocratique est presque tout entière enfermée dans le processus électoral - au milieu de longues périodes passives ${ }^{403}$.

On sait que la France politique, depuis la Révolution, est hostile aux groupes intermédiaires, corporations ou états de sinistre mémoire (je veux dire, de mémoire aristocratique). La république, en France, ne connaît que le citoyen. Le frémissement que l'on nous annonce vers une « démocratie participative » est porteur d'une révolution en ce domaine, lié qu'il est à la prise en compte de communautés de citoyens.

\section{Le soutien public}

Les Public Libraries sont nées d'en-bas, de la volonté de quelques voisins (A few neighbors joined together, Jeremy Belknap). Les bibliothèques municipales sont créées par les pouvoirs publics, sans que la population les demande - on peut dire, en forçant le trait, qu'elles sont octroyées d'enhaut. Les contribuables américains donnent leur avis sur le financement de leurs bibliothèques : ils votent dans des referendums pour accepter (ou refuser) des ressources nouvelles, ils donnent (ou ne donnent pas) pour que les contributions privées accompagnent et complètent les financements publics. Les habitants donnent aussi de leur temps, soit dans les associations d'amis, soit comme volontaires. Ils se mobilisent quand les services de la bibliothèque, ou son existence même, sont menacés.

Rien de tout ceci en France. Le financement des bibliothèques municipales est pris en charge par l'impôt (et, marginalement, par les usagers) sans que les contribuables aient à se prononcer sur son montant ou son affectation. Les bibliothèques municipales existent indépendamment de leurs usagers, de leur demande et de leur satisfaction. Indépendamment de leur soutien. Ce qui signifie non seulement le primat de la politique d'offre (qui a, par ailleurs, sa justification intellectuelle) mais aussi que, lorsque les bibliothèques municipales ont besoin d'être soutenues, quand elles sont instrumentalisées, délaissées, abandonnées, les usagers, contribuables et citoyens ne les défendent pas. Leur seule défense est corporatiste - celle que les bibliothécaires leur apportent.

403. On pourrait utiliser l'image d'une démocratie en marche, une démocratie d'acteurs, comme les Américains le font lors de la parade du 4 juillet, et d'une démocratie qui regarde, une démocratie d’observateurs, comme les Français le font lors du défilé du 14 juillet. 


\section{L'advocacy}

Le soutien aux Public Libraries est une sorte de genre littéraire. Parler pour les bibliothèques, porter témoignage de leur importance, souligner leur apport, leur place dans la société américaine sont des exercices de style, auxquels se livrent les présidents des États-Unis comme les élus, les usagers ou les bibliothécaires. La promotion des bibliothèques, tout au long de l'année au sein des Amis de la bibliothèque ou dans un moment particulier comme la National Library Week, est une activité commune, courante. Elle inspire tout naturellement le soutien public que les Public Libraries entretiennent comme le propriétaire anglais entretient son gazon. Rien de tel en France, est-il encore besoin de le souligner. Au contraire, une rhétorique de circonstance - comme il y a des discours de circonstance - de la part des décideurs ; et, de la part des bibliothécaires, un soupçon permanent que les bibliothèques ne remplissent pas leur rôle. Le tout, baignant dans un flou savant sur ce qu'est leur rôle.

\section{LE RAPPORT AU SAVOIR}

Ces quatre divergences majeures sont les conséquences, les signes manifestes d'une origine différente. Mais elles ne l'épuisent pas tout à fait. Si l'on prolonge l'analyse, au-delà du constat, les raisons de ces divergences se trouvent aussi dans le rapport au savoir - savoir utile, savoir confisqué, savoir partagé... Avec l'aide de deux historiens français, Jean Hassenforder et Alexis de Tocqueville, tentons d'aller plus loin dans cette analyse.

\section{Le peuple, l'aristocratie et le savoir}

Reprenons d'abord l'analyse de Jean Hassenforder ${ }^{404}$. Aux États-Unis : " La participation de tous aux affaires politiques, suppose la diffusion des connaissances requises, une généralisation de l'instruction. L'enseignement public s'affirme effectivement aux États-Unis dans la première moitié du siècle. Des bibliothèques destinées à la fois aux enfants et aux adultes, apparaissent dans les écoles, dans l'État de New York à partir de 1835,

404. Jean Hassenforder. Développement comparé des bibliothèques publiques en France, en GrandeBretagne et aux États-Unis, op. cit., p. 90 et 93-94. 
puis dans d'autres états. Financées par des fonds publics, elles ouvrent la voie, sur ce point, aux bibliothèques municipales".

"Les aspirations démocratiques sont invoquées fréquemment à l'appui de la création de ces dernières. Le rapport des premiers responsables de la bibliothèque publique de Boston est explicite à cet égard. C'est le problème d'une démocratisation de l'accès à la connaissance qui est posée : "Il est de suprême importance que les moyens d'une information générale soient ainsi diffusés, que le plus grand nombre possible de personnes soit amené à lire et à comprendre les questions concernant les fondements mêmes de l'ordre social qui se présentent constamment à eux et sur lesquels on demande constamment au peuple de décider." Offrir à tous cette possibilité de lecture est un devoir des pouvoirs publics au même titre que l'apport d'une éducation gratuite et en fait comme une partie et des plus importantes de cette éducation à l'intention de tous. Cette conviction anime effectivement les pionniers de la bibliothèque municipale de Boston. La conception aristocratique de la bibliothèque au seul usage d'une minorité lettrée est répudiée et l'on s'efforce d'offrir à tous un service diversifié, sans oublier de prendre en considération les besoins des plus pauvres. La distinction européenne entre bibliothèques populaires et bibliothèques savantes s'efface au profit d'une conception plus large de la bibliothèque publique. »

Inversement, en France, poursuit Hassenforder, « La Révolution française a mis les bibliothèques publiques à l'ordre du jour. Mirabeau, Condorcet, Talleyrand évoquent le problème, et elles trouvent place dans des projets inspirés par la généreuse idée de répandre l'instruction et d'en assurer l'accès à tous. Des bibliothèques publiques sont créées, qui rassemblent des livres nombreux en provenance de biens saisis. Mais la richesse initiale des bibliothèques municipales ne sera pas sans contrepartie. L'accent mis sur des fonds vieillis, utiles aux érudits et aux historiens, se révélera par la suite un obstacle à une meilleure adaptation de ces institutions à la vie moderne. »

C'est, en une page, résumer la faille initiale qui sépare les bibliothèques publiques aux États-Unis et en France : une naissance démocratique làbas, un héritage aristocratique ici.

Aux États-Unis, la liberté d'information est la valeur nodale, celle qui est défendue bec et ongles à travers tout le pays. En France, on peut dire, sans excès, que le sujet n'est pas un sujet sensible ; ce qui est en question, c'est que les usagers potentiels ne soient pas exclus, rejetés. Que le trésor soit accessible à tous. Comme si les bibliothèques des émigrés 
devaient, une nouvelle fois, être ouvertes et mises à disposition du peuple. Les bibliothèques américaines n'ont pas ce souci, nées dans le peuple et pour lui.

Ce serait sans doute forcer le trait (quoique...) que d'écrire que, de part et d'autre de l'Atlantique, chacun rejoue la scène originelle, les uns, nés égaux, citoyens, hommes d'action, jaloux de leur liberté, les autres, jadis sujets, spectateurs ironiques, attachés à ne pas être exclus, soucieux d'un partage équitable. " Le grand avantage des Américains est d'être arrivés à la démocratie sans avoir à souffrir de révolutions démocratiques, et d'être nés égaux au lieu de le devenir. ${ }^{405}$ »

Cette faille, identifiée et analysée par Jean Hassenforder pour le XIX siècle, ne s'est pas résorbée au cours du $\mathrm{xx}^{\mathrm{e}}$ siècle. Pourquoi ? Les raisons en sont complexes - j'en ai évoqué quelques-unes, l'héritage des bibliothèques savantes, la prégnance des collections littéraires, le refus du rôle éducatif, le rôle de l'État dans la définition du paradigme, le goût pour la modernité. Ici, il convient de creuser l'analyse.

\section{Un savoir utile?}

Peuple de pionniers, d'individualistes, d'entrepreneurs, de self-made men, les Américains, on le souligne volontiers, ne prisent pas les grandes idées, la théorie, les penseurs. Pragmatisme semble être leur maître-mot. Mais le savoir est une valeur sûre. Car il conditionne la réussite, comme l'analysait déjà Tocqueville : « Quand il n’y a plus de richesses héréditaires, de privilèges de classes et de prérogatives de naissance, et que chacun ne tire plus sa force que de lui-même, il devient visible que ce qui fait la différence entre la fortune des hommes, c'est l'intelligence. Tout ce qui sert à fortifier, à étendre, à orner l'intelligence acquiert aussitôt un grand prix. ${ }^{406}$ " L'accès au savoir est une condition essentielle de l'égalité des chances. Un savoir utile car prédomine « dans le cœur de l'homme le goût de l'utile sur l'amour du beau », écrit encore Tocqueville.

Mais qu'est-ce qu'un savoir utile ? Un savoir utile à l'action, des livres qui se lisent vite, « qui n'exigent point de recherches savantes pour être compris ». Un savoir contemporain : "Les peuples démocratiques n'ont qu'une estime fort médiocre pour l'érudition et ils ne se soucient guère de ce qui se passait à Rome et à Athènes; ils entendent qu'on leur parle 
d'eux-mêmes et c'est le tableau du présent qu'ils demandent. ${ }^{407}$ » En des termes d'un autre siècle, il s'agit bien là de lecture d'information et de formation. Des livres pour comprendre et agir.

Symétriquement, les bibliothèques françaises possèdent des collections dont la qualité première n'est pas l'utilité immédiate. Ce qui est valorisé par les bibliothécaires, c'est la cohérence et la richesse du corpus constitué. Ce sont les fonds littéraires ${ }^{408}$. Les usagers, eux, on l'a vu, considèrent qu'ils n'y trouvent pas forcément leur miel - d'abord et surtout pour les livres pratiques, mais aussi pour les livres scientifiques et de sciences humaines et sociales. La richesse de fonds constitués dans l'épaisseur du temps est inappréciable. Mais un intérêt insuffisant pour les idées et les sciences d'aujourd'hui ne saurait réconcilier les Français et leurs bibliothèques.

Pour autant, la pente américaine à acquiescer à toute la production éditoriale, quel que soit son contenu ${ }^{409}$, est absolument étrangère à la tradition française. La constitution des collections se fait par sélection, une sélection assumée qui prend en compte l'actualité éditoriale, la collection déjà là (ses points forts, ses lacunes, ses redondances, la nécessité de la mettre à jour), les attentes de la population et l'usage possible des livres achetés. Mais ce critère (le succès des livres achetés), en France, passe en dernier. L'achat par la New York Public Library, en 2005, de 1055 exemplaires de Harry Potter and the Halfblood Prince ne peut provoquer chez les bibliothécaires français qu'une question incrédule et ironique : mais qu'est-ce qu'ils en feront après, quand ce titre ne sera plus dans l'actualité médiatique ? Inversement, un livre qui n'est pas emprunté, pas lu, n'est pas forcément considéré comme n'ayant pas sa place à la bibliothèque : il pourra trouver un lecteur plus tard.

407. Ibid., II, p. 115.

408. L'analyse suivante, si familière sur le fond aux bibliothécaires français, n'aurait pas de sens aux États-Unis : « Le premier danger qui guette les fonds de littérature est la standardisation, l'uniformisation. Les beaux fonds, ceux qui ont une couleur, une densité, sont ceux qui contiennent un certain mystère. On va y rencontrer des lignes de force, des résistances, des motifs de surprise et des incitations à la découverte. La diversité de la production doit inciter les professionnels à sortir des sentiers battus et à construire des collections diversifiées. Pas seulement pour répondre aux attentes supposées des différentes catégories de lecteurs. C'est une question de politique de l'offre, et même de politique culturelle. » (Madeleine Deloule. « Acquisitions, classement, mise en valeur ». Littérature contemporaine en bibliothèque, sous la dir. de Martine Poulain. Éditions du Cercle de la Librairie, 2001)

409. Gary Strong, directeur de la Queens Library, à New York : " Tout tient au suivi de la satisfaction de nos usagers, qui consiste à savoir précisément ce que veulent nos clients et à le leur donner. » (Gary Strong, " Relever le défi de la diversité à la Queens Library », Bulletin des bibliothèques de France, t. 47, n 1, 2002) 
Si l'inutilité des collections n'est pas, évidemment, reconnue comme souhaitable, un certain désintéressement est volontiers prôné. Une certaine gratuité. Conséquence, là aussi, de la déconnexion de la bibliothèque et de la population. La satisfaction immédiate du public n'est pas le critère premier, en France, pour la constitution des collections. Mais cette gratuité est aussi la conséquence d'un autre rapport au temps, à l'actualité (voire à la médiatisation), à l'utilité immédiate. Les bibliothèques municipales, plus que les Public Libraries, sont inscrites dans le temps long, dans l'épaisseur du temps.

\section{Un savoir populaire ?}

« Elitiste pour tous » : l'oxymore (attribué à Hölderlin, Vitez et quelques autres) n'éclaire pas l'analyse. On voit bien sa fonction : éviter le mot honni de «populaire ». Le Théâtre national populaire l'est (« populaire ») dans le bon sens : il est exigeant pour le peuple et n'est pas condescendant. On se souvient de l'éructation d'Eugène Morel contre les bibliothèques dites " populaires » : «Il y a le mot "populaire". Théâtre populaire, université populaire, restaurant populaire, bibliothèque populaire... C'est avec ce mot-là que les meilleures intentions dressent de suite une insulte devant l'œuvre qu'elles entreprennent. » Dans l'action populaire, il y a au pis un peu de mépris, au mieux un peu de paternalisme. Entre l'élitisme et le paternalisme, la voie est étroite.

C'est cette voie que veut suivre la « bibliothèque publique » prônée par les bibliothécaires modernistes du $\mathrm{Xx}^{\mathrm{e}}$ siècle. Ni bibliothèque savante pour les savants, ni bibliothèque populaire pour le peuple, la bibliothèque publique veut casser ces modèles d'exclusion et accueillir tous et chacun. C'est ce que décrit Michel Bouvy : "L'idée de la bibliothèque pour l'élite et celle de la bibliothèque populaire, ce sont les deux idées qui ont toujours fait le plus de mal aux bibliothèques françaises, et malheureusement ce sont les plus répandues à l'heure actuelle dans la plupart des milieux. La bibliothèque publique n'est, il faut le répéter, ni un service de luxe ni une œuvre de bienfaisance. C'est un service public aussi utile que l'école. » Quarante ans après ces déclarations d'intention, il considère que la médiathèque à la française a répondu à cette attente : « Je ne connais pas de médiathèque d'étude et de médiathèque populaire. Les médiathèques sont ouvertes à tous. ${ }^{410}$ " 
On peut pourtant s'interroger, à nouveau, sur le rapport des bibliothèques au peuple - c'est-à-dire à la société. Et souligner, à nouveau, l'écart entre l'exemple français et l'exemple américain. Dès le XIX ${ }^{\mathrm{e}}$ siècle, l'accès au savoir est considéré aux États-Unis comme une nécessité pour tous, et ce d'autant plus que l'école ne peut suffire à un partage équitable du savoir. Pas de savants ni d’ignorants : c'est ce que constate Tocqueville : «Celui qui veut juger quel est l'état des lumières parmi les Anglo-Américains est donc exposé à voir le même objet sous deux différents aspects. S’il ne fait attention qu'aux savants, il s'étonnera de leur petit nombre ; et s'il compte les ignorants, le peuple américain lui semblera le plus éclairé de la terre. ${ }^{411}$ »

Martine Poulain, dans sa relecture de Tocqueville ${ }^{412}$, revient sur la question du partage du savoir dans la jeune démocratie américaine du $\mathrm{XIX}^{\mathrm{e}}$ siècle. «Seule une société démocratique pouvait inventer la lecture publique : sans patrimoine et sans passé, cette société avait besoin, pour construire, nourrir et développer son fonctionnement égalitaire et individualiste, de journaux et de bibliothèques, alors sources essentielles du savoir. » La différence organique avec l'histoire française a, on y revient, à voir avec la culture religieuse. Aux États-Unis, il s'agit de proposer librement le savoir pour que chaque lecteur acquière les connaissances qui lui sont utiles et forge sa propre opinion. " Le but premier des bibliothèques publiques américaines », écrit encore Martine Poulain, « était d’informer plus que de former, de fournir des informations plus que des corpus, des connaissances plus que des savoirs, des faits plus que des pensées. »En France, à la même époque, le lecteur, s’il n'est un savant, est considéré comme un mineur qu'il faut guider, encadrer, et dont les lectures doivent être contrôlées. La prescription, si elle a disparu du vocabulaire français, demeure l'arrière-plan de la constitution des collections. Tradition protestante, tradition catholique...

Aux États-Unis, nulle position de surplomb : « Les nouvelles bibliothèques créées par la démocratie américaine ne pouvaient donc pas, à la différence de ce qui se passe dans les vieilles sociétés européennes à la même époque, être populaires, c'est-à-dire s'adresser à une partie de la population désignée comme étant le peuple, différente des autres classes

411. Alexis de Tocqueville. De la démocratie en Amérique, op. cit., I, p. 446.

412. Martine Poulain. "Retourner à Tocqueville pour comprendre l'histoire comparée des bibliothèques américaines et françaises au XIX ${ }^{\mathrm{e}}$ siècle », Bulletin des bibliothèques de France, t. $47, \mathrm{n}^{\circ} 5$, 2002. 
sociales et nécessitant un traitement précautionneux particulier dans sa progression vers la lumière des connaissances ", conclut Martine Poulain. La conception holiste de la société américaine, la société sans classes des idéologues jeffersoniens ${ }^{413}$, a donné naissance aux Public Libraries. La société française, hiérarchisée, de classes, a engendré des bibliothèques municipales, octroyées à la population et au fonctionnement encore largement aristocratique ${ }^{414}$. Pourtant, le projet de la bibliothèque publique est largement démocratique. Comment comprendre cet écart entre le projet et la réalité ?

\section{DEUX PROJETS DIFFÉRENTS}

Revenons sur le constat que l'importation de la Public Library en France a été infidèle au modèle importé. Le modèle mis en œuvre sous le nom de « bibliothèque publique " s'est développé sous la forme de la médiathèque. La médiathèque est un modèle de rupture avec la bibliothèque savante ; ses maîtres-mots sont " modernité » et " ouverture ». C'est par la modernisation des établissements que se fera, dit ce modèle, l'ouverture (aux différents publics). Nous venons de rappeler en quoi ce modèle diverge de la Public Library - dont il se réclame pourtant.

Tentons un pas de plus dans l'analyse en examinant en quoi, dans sa version contemporaine, ce modèle francisé peut être fidèle à un projet démocratique. Il l'est dans le registre de la redistribution des ressources (le savoir, les informations, la culture, le patrimoine : la bibliothèque comme bien public à partager) voire, ce social n'étant pas appuyé sur un projet politique, dans le registre caritatif (la bibliothèque comme octroyée à la population, et d'abord dévolue à la population qui en est exclue).

Le souci de faire venir à la bibliothèque en priorité la population qui en est éloignée peut, en effet, être considéré comme le marqueur d'une approche caritative. Le caritatif contre le politique ? Ou, plutôt, le caritatif car sans le politique ${ }^{415}$ ? Si la médiathèque n'est pas un projet poli-

413. Elise Marienstras. Les mythes fondateurs de la nation américaine. Éditions Complexe, 1992 , p. 26.

414. Je renvoie sur ce point au développement sur la place des usagers dans les bibliothèques, au chapitre III. On m'objectera que la société anglaise, dont les bibliothèques sont proches des américaines, est très hiérarchisée. Alors, c'est sans doute qu'à cette hypothèse à partir de la structure sociale il est nécessaire d'ajouter le facteur religieux. Le libre examen (protestant) des idées, des textes, des grands récits exige une bibliothèque ouverte. Démocratique. 
tique, elle a cependant des fondements qui spécifient sa place dans l'expace public. Deux types de remarque peuvent être apportés pour éclairer cette analyse. Une remarque sur le rapport au temps, une remarque sur le modèle qu'est la médiathèque.

\section{La médiathèque et le temps des lecteurs}

Contrairement aux bibliothécaires américains, la culture professionnelle des bibliothécaires français intègre une dimension téléologique. Le nonusager est un futur usager ; l'usager incompétent est un futur usager expert ; l'enfant lecteur est l'usager de demain ; le lecteur de romans policiers est un futur lecteur de (au choix) Proust ou Joyce. La prescription des bons livres a formellement disparu mais l'idée d'un itinéraire de lecteur, avec une intensification de la pratique et une complexification des lectures, reste présente. Car les bibliothécaires aiment les lecteurs pour ce qu'ils pourraient devenir - pour ce qu'ils pourraient lire peut-être plus que pour ce qu'ils lisent ${ }^{416}$. La métaphore du passage (d'une lecture enfantine à une lecture adulte, d'une lecture facile à une lecture difficile) est fréquente dans l'offre des bibliothèques. Le self-improvement américain est, chez nous, vu comme relevant de l'institution (la bibliothèque) et de ses acteurs (les bibliothécaires) plus que de l'individu lisant.

C'est sans doute que la tradition de self-improvement correspond en France à la tradition d'émancipation. Ici, on lit pour se libérer (des oppressions, des héritages, des contraintes) non (comme là-bas) pour devenir

415. L’objectif fréquemment assigné aux bibliothèques d'entretien du lien social ou de facilitation de l'insertion sociale est un des fondements de la légitimation des établissements aujourd'hui. Il est aussi analysé comme un acquiescement, une résignation à des activités de contrôle social qui prendraient le pas sur l'émancipation. Ainsi, Bruno David : «Si on laisse de côté (provisoirement) les ritournelles sur "la démocratisation de la culture" - passage obligé du grand oral que sont les discours d'inauguration d'équipements -, on n'ignore pas que les élus locaux envisagent la création de médiathèques comme une réponse parmi d'autres aux risques de "désaffiliation" (Castel) et d'éclatement social qui menacent "les quartiers sensibles" de leur commune. Autrement dit, ces équipements s'intègrent à la géographie urbaine en tant que dispositifs d'encadrement social - aux côtés du sport (entre autres), souvent présenté comme une riposte douce au désœuvrement criminogène des jeunes. Or, ce qui pour les politiques est une nécessité dictée par la gestion des risques sociaux (notamment celui que représentent les classes dangereuses), les bibliothécaires en font une vertu civique - un état d'esprit qui présuppose l'identification à l'ordre et l'intériorisation de ses valeurs. » (Bruno David, « Le manège enchanté des bibliothécaires », Bulletin des bibliothèques de France, t. 49, $\mathrm{n}^{\circ}$ 6, 2004)

416. Dans un bref texte autobiographique, Dominique Tabah se souvient de " la thèse d'Hélène Cixous sur Joyce achetée en six exemplaires » (" Mémoire de la territoriale : les communaux arrivent », BIBLIOthèques, $\left.n^{\circ} 28,2006\right)$. Dans ma propre expérience, j'ai rencontré des bibliothécaires proposant tout Lacan dans une très petite bibliothèque d'un quartier défavorisé, argumentant que même les gens de ce quartier « avaient le droit de lire Lacan ». 
plus compétent, plus débrouillard ou plus riche ${ }^{417}$. C'est ce discours de l'émancipation qui a appuyé le mouvement de développement des bibliothèques dans les années 1970 et 1980 dans certaines villes, en particulier les villes communistes ${ }^{418}$. C'est lui que l'on retrouve en filigrane dans certains travaux sociologiques ${ }^{419}$. C'est lui dont la disparition a probablement provoqué l'incertitude politique qui marque les fondements des bibliothèques publiques. Car ce n'est pas ce discours qui fonde le modèle de la médiathèque.

Laissons Michel Melot décrire ce modèle : « Le concept de "médiathèque" ne saurait se réduire à l'intégration des nouveaux médias dans le dépôt de livres. La médiathèque répond beaucoup plus largement à l'idée d'une bibliothèque ouverte non seulement à tous les types de documents mais aussi à tous les publics, et à toutes sortes d'activités d'information ou de loisirs. Son succès est donc entièrement lié au développement des pratiques bibliothécaires nouvelles en France, bien connues des Public Libraries, anglo-saxonnes, comme le libre accès, l'information et la documentation, le lieu civique ou convivial de rencontres et d'animations, bref, tout ce qui peut la distinguer de la vieille bibliothèque savante réservée aux notables et aux érudits, dont elle a servi à faire le procès et à dépasser le modèle. ${ }^{420}$ » Si la médiathèque permet/promet une émancipation, une rupture, une désaffiliation, c'est celles d'avec la bibliothèque savante et la culture légitime.

\section{Le modèle de médiathèque}

Sur quel autre fondement politique que celui de la démocratisation (au sens de fréquentation par des personnes aujourd'hui éloignées de la bibliothèque), s'appuie alors le modèle de la médiathèque ? On peut identifier deux autres registres : celui du service public (« à la française » selon la formule désormais consacrée), celui du réseau (valable, lui, en France comme aux États-Unis).

417. Myriem Foncin, dans la critique de The Chance to read : « Il est bien certain que des bibliothécaires français n'accepteront pas en bloc la définition que M. McColvin donne de la bibliothèque de lecture publique, définition qu'il emprunte à Robert D. Leigh. La plupart, en tout cas, intervertiront l'ordre des facteurs, assignant comme premier objectif à la bibliothèque l'enrichissement personnel de l'individu et non sa formation civique et sociale. » (Bulletin d'informations de l'ABF, $\left.\mathrm{n}^{\circ} 23,1957\right)$

418. Sur cette argumentation, je renvoie à ma thèse, Anne-Marie Bertrand. Les villes et leurs bibliothèques : légitimer et décider (1945-1985), op. cit., p. 275-277.

419. Michèle Petit, Chantal Balley. De la bibliothèque au droit de cité : parcours de jeunes. BPI, 1996.

420. Michel Melot. « Le temps des médiathèques », op. cit., p. 209. 
Les bibliothèques publiques sont un service public, émanant de collectivités publiques, assurant un service d'intérêt général au bénéfice de l'ensemble de la population. Cette phrase pourrait décrire aussi l'activité des Public Libraries mais ne serait sans doute pas considérée comme essentielle, identitaire, alors qu'elle est un substrat intériorisé en France. $\mathrm{Au}$ temps des Trente Glorieuses, travailler « dans le public » est un choix - quasiment un choix de vie. Les bibliothèques sont, alors, porteuses de cette foi, ainsi définie par Bernadette Seibel : « L'ensemble de la profession partage la croyance que la connaissance est bonne en soi et qu'il lui appartient de transmettre à tous les savoirs ou biens culturels. ${ }^{421}$ » Outre l'objectif de partage, inhérent à toute action culturelle publique, les acteurs qui font ce choix le font aussi parce qu'il y a " une certaine noblesse à n'être au service que de l'intérêt général, et à traiter avec bienveillance, conformément à son propre sens du devoir, des usagers qui du fait qu'ils ne peuvent faire appel à un concurrent, sont mal placés pour exiger. ${ }^{422}$ » Le service public est un choix noble, qui plus est compatible avec la faible culture de service française. Ce qui le rend d'ailleurs doublement incompréhensible pour les Américains.

Mais le service public a changé. Les mythes qui le fondaient se sont délités. La noblesse de servir est dépassée par le souci de répondre aux attentes. La satisfaction des usagers est devenue la mesure de toute activité publique.

Dans cette nouvelle articulation entre population, usagers et fonctionnaires, dans cette nouvelle incertitude créée par l'offre privée (et domestique) qu'est Internet, dans cette renégociation de l'utilité sociale des bibliothèques, de quelle justification nouvelle peut se réclamer la médiathèque ? La modernité est plus que jamais à l'ordre du jour. Modernité d'un monde informationnel, flexible, nomade. Denis Varloot, dans un article anticipateur resté célèbre, indiquait la voie dès 1983 : " Plus brève, l'information sera beaucoup plus mobile, plus dynamique et, pour partie, moins pérenne peut-être. C'est cette information libérée que sollicitent ceux qui ne sont encore aujourd'hui que des lecteurs. » Il incitait au changement, pour répondre à cette nouvelle attente, « afin que, de "gardiens du coffre” qu'ils sont aujourd'hui, les bibliothécaires deviennent des spécialistes de l'irrigation, de ces "fontainiers" qui font pousser des arbres jusque dans le désert. ${ }^{423}$ » 
La « boîte à livres » qu'est la bibliothèque est appelée à devenir un élément du vaste réseau que constitu(erai)ent aujourd'hui nos sociétés occidentales. Accès, accessibilité, accéder : ces mots emplissent, saturent la rhétorique récente : les bibliothèques seraient devenues ou sommées de devenir des points de passage, des nœuds dans un réseau, et les bibliothécaires ces «fontainiers " que Denis Varloot appelait de ses vœux. L'hédonisme d'aujourd'hui, avec ses valeurs de rapidité, de jeu, de convivialité, ne se reconnaît pas dans la médiathèque. Le flux, le « juste-àtemps », l'éphémère, sont en opposition avec la durée, la lenteur, le stock des bibliothèques. Pour répondre aux nouvelles attentes de la société, la bibliothèque devrait se faire immatérielle, fluide, gazeuse. Aux ÉtatsUnis, l'existence du réseau est supposée avoir provoqué la désertion des bibliothèques - mais les statistiques démentent l'analyse, du moins pour les Public Libraries ${ }^{424}$.

La création de services à distance, la personnalisation des prestations, la focalisation sur l'accès (et non plus sur l'acquisition) sont supposées permettre la présence de la médiathèque dans le réseau (global, moderne, interactif) - nouvelle fiction, elle aussi, sans fondement politique.

Michel Melot prédit la fin de la médiathèque : "La médiathèque est devenue une évidence. En conséquence, le mot même de "médiathèque", qui assura pendant un demi-siècle la promotion des bibliothèques publiques en France, perd de son efficacité et la "bibliothèque" revient à la mode, dégagée de ses connotations passéistes. L'époque des "médiathèques" peut alors apparaître dans l'histoire des bibliothèques françaises comme un long et décisif détour. ${ }^{425}$ » Mais un détour qui ramène à quoi ?

Ce travail d'analyse comparée entre les Public Libraries et les bibliothèques municipales ouvre ainsi - et c'est heureux ! - sur de nouvelles interrogations et, nécessairement, sur de nouvelles recherches. Au moins dans deux registres.

D'une part, il conviendrait de revenir sous un autre angle sur la question (ici peu, mal traitée) de l'adaptation du modèle américain. Comment le transfert culturel s'est-il effectué, voilà un sujet que je n'ai abordé que sous l'angle de l'adaptation (ici infidèle) du modèle. Le rôle des "passeurs culturels », des vecteurs de cette importation, des raisons et des

424. Pour la désertion : Scott Carlson. "The Deserted Library ». The Chronicle of Higher Education, 16 novembre 2001. Pour la stabilité des chiffres dans les Public Libraries, Philippe Cantié, $A u$ nom de l'antiterrorisme, op. cit., p. 104-105.

425. Michel Melot, « Le temps des médiathèques », op. cit. 
modalités de l'adaptation de ce modèle à son espace d'importation est à reprendre.

D’autre part, l'histoire (très) contemporaine des bibliothèques amène à s'interroger sur la question des modèles. Si le modèle américain a été importé fautivement, il a bel et bien joué un rôle important dans la définition de la médiathèque. Cette hybridation a produit un artefact spécifiquement français explicité par une rhétorique insistante (la modernité, la démocratisation) qui prend aisément, complaisamment, les mots pour les choses. La théorie de la médiathèque reste à faire. La comparaison avec la Public Library montre ce qu'elle n'est pas. Le discours qu'on tient sur elle dit ce qu'elle prétend être. Une théorie des bibliothèques, une théorie de la pratique, devrait montrer ce qu'elle est.

L'histoire culturelle est un outil indispensable dans cette théorisation. Elle permet de replacer la bibliothèque dans son espace public, dans sa tradition politique, culturelle, sociale, religieuse. Elle est un nécessaire outil d'intelligibilité car elle peut prendre en compte à la fois les héritages et les projets, les discours et les faits, les promesses et les réalisations. Elle permet d'analyser et de comprendre en quoi la bibliothèque est un artefact - et cet artefact-là. 


\section{BIBLIOGRAPHIE}

\section{ANALYSE}

\section{Monographies}

L'Amérique dans les têtes. Sous la dir. de Denis Lacorne. Hachette, 1986.

Barnett Graham K. Histoire des bibliothèques publiques en France de la Révolution à 1939. Promodis, 1987.

Bertrand Anne-Marie. Bibliothécaires face au public. BPI, 1995.

Bertrand Anne-Marie. Les villes et leurs bibliothèques : légitimer et décider (19451985). Éditions du Cercle de la Librairie, 1999 (Bibliothèques).

Bertrand Anne-Marie, Burgos Martine, Poissenot Claude, Privat Jean-Marie. Les bibliothèques municipales et leurs publics. BPI, 2001.

Bobinski George. Carnegie Libraries: Their History and Impact on American Public Library Development. ALA, 1969.

Cantié Philippe. Au nom de l'antiterrorisme : les bibliothécaires américains face à l'USA Patriot Act. Presses de l'enssib, 2006.

Chartier Anne-Marie, Hébrard Jean. Discours sur la lecture (1880-2000). BPIFayard, 2000.

Damien Robert. Bibliothèque et État : naissance d'une raison politique dans la France du XVII siècle. PUF, 1995 (Questions).

Damien Robert. Le conseiller du Prince de Machiavel à nos jours : genèse d'une matrice démocratique. PUF, 2003 (Fondements de la politique).

Davis Donald G. Jr, Tucker John M. American Library History: A Comprehensive Guide to the Literature. ABC-CLIO, 1989.

Ditzion Sidney H. Arsenals for a Democratic Culture: A Social History of the American Public Library Movement in New England and the Middle States from 1850 to 1900. ALA, 1947. 
Eisenstein Elizabeth L. La révolution de l'imprimé à l'aube de l'Europe moderne. Hachette, 1991 (Pluriel).

Gauchet Marcel. Le désenchantement du monde. Gallimard, 1985 (Folio).

Gaudet Françoise, Lieber Claudine. Les ressources électroniques dans les bibliothèques américaines, [rapport Bourse Fulbright], 2002.

Glassberg David. Sense of History, the Place of the Past in American Life. University of Massachusetts Press, 2001.

Harris Michael H. History of Libraries in the Western World. The Scarecrow Press, 4th ed., 1995.

Hassenforder Jean. Développement comparé des bibliothèques publiques en France, en Grande-Bretagne et aux États-Unis dans la seconde moitié du XIX siècle (18501914). Éditions du Cercle de la Librairie, 1967.

L'histoire culturelle du contemporain. Sous la dir. de Laurent Martin et Sylvain Venayre. Nouveau Monde Éditions, 2005.

Histoire des bibliothèques françaises. Tome IV : les bibliothèques au XX siècle (19141990). Sous la dir. de Martine Poulain. Promodis-Éditions du Cercle de la Librairie, 1992.

Hoffmann Stanley. Essais sur la France : déclin ou renouveau ? Éditions du Seuil, 1974 (Esprit).

Hofstadter Richard. Anti-Intellectualism in American Life. Knopf, 1963.

Kaspi André. Les États-Unis d'aujourd'hui, mal connus, mal aimés, mal compris. Perrin, 2004.

Kriegel Blandine. Philosophie de la République. Plon, 1998.

Kuhlmann Marie, Kuntzmann Nelly, Bellour Hélène. Censure et bibliothèques au XXe siècle. Éditions du Cercle de la Librairie, 1989.

Lajeunesse Marcel. Lecture publique et culture au Québec, XIX et XX $X^{e}$ siècles. Presses de l'université du Québec, 2004.

Library History Research in America: Essays Commemorating the Fiftieth Anniversary of the Library History Round Table, ed. by Andrew B. Wertheimer and Donald G. Davis Jr. ALA, 2000.

Maresca Bruno, Evans Christophe, Gaudet Françoise. Les bibliothèques municipales au tournant d’internet. BPI, 2007. 
Marienstras Elise. Les mythes fondateurs de la nation américaine. Éditions Complexe, 1992 (Historiques).

Melot Michel. « Pour une géopolitique des bibliothèques ». In Anne-Marie Bertrand, Anne Kupiec, Ouvrages et volumes : architecture et bibliothèques. Éditions du Cercle de la Librairie, 1997 (Bibliothèques).

Mény Yves, Surel Yves. Politique comparée : les démocraties, Allemagne, États-Unis, France, Grande-Bretagne, Italie. Montchrestien, 6éd., 2001.

Morel Eugène. Bibliothèques. Mercure de France, 1909.

Morel Eugène. La librairie publique. Armand Colin, 1910.

Munthe Axel. American Librarianship from an European Angle: An Attempt at an Evaluation of Politics and Activities. ALA, 1939.

Naudé Gabriel. L'Advis, manifeste de la bibliothèque érudite, éd. par Claude Jolly. Aux amateurs de livres, 1990.

Quel modèle de bibliothèque ? Sous la dir. d'Anne-Marie Bertrand. Presses de l'enssib, 2008, 183 p.

Regards sur un demi-siècle : cinquantenaire du Bulletin des bibliothèques de France, $B B F, 2006$.

Richter Noë. La lecture \& ses institutions (1919-1989). Plein chant, 1989 (L’Atelier furtif).

Richter Noë. Introduction à l'histoire de la lecture publique. Éditions de la Queue du chat, 1995.

Roger Philippe. L'ennemi américain. Éditions du Seuil, 2002 (La couleur des idées).

The Role of Libraries in America: A Report of the Survey conducted by the Gallup Organization. Kentucky Department of Library and Archives, 1976.

Rosanvallon Pierre. L'État en France de 1789 à nos jours. Éditions du Seuil, 1990 (L’Univers historique).

Rouet François. La grande mutation des bibliothèques municipales : modernisation et nouveaux modèles. Ministère de la Culture, 1998.

Schnapper Dominique. La démocratie providentielle : essai sur l'égalité contemporaine. Gallimard, 2002 (NRF Essais).

Shera Jesse. Foundations of the Public Library: The Origins of the Public Library Movement in New England (1629-1855). The Shoe String Press, 1965. 
Steiner George. Une certaine idée de l'Europe. Actes Sud, 2005.

Tocqueville Alexis de. De la démocratie en Amérique. 2 vol. Gallimard, 1961 (Folio).

\section{Articles}

Bazin Patrick. « Après l'ordre du livre ». Medium, n 4, 2005.

Bertrand Anne-Marie. « Le peuple, le non-public et le bon public : les publics des bibliothèques et leurs représentations chez les bibliothécaires ». Le(s) public(s) de la culture, sous la dir. d'Olivier Donnat et Paul Tolila. Presses de Sciences Po, 2003.

Butlen Max. " Lire en bibliothèque, lire à l'école : oppositions et interactions ». Bulletin des bibliothèques de France, t. 49, n 1, 2004, p. 5-10.

Carpenter Kenneth, Augst Thomas. "The History of Libraries in the United States: A Conference Report”. Libraries \& Culture, vol. 38, n 1, Winter 2003, p. 61-65.

Colson John C. “The Writing of American Library History (1876-1976)”. Library Trends, July 1976, p. 7-22.

Goedeken Edward A. "What We Wrote About and Who We Were: Historical Writings in JLH/L \& C, 1966-2000”. Libraries \& Culture, vol. 38, n 3, Summer 2002, p. 250265.

Hassenforder Jean. «Un mouvement pour le développement des bibliothèques publiques : les origines de la Section des bibliothèques publiques de l'Association des bibliothécaires français ». Médiathèques publiques, nº 71-72, 1988.

Kessler Jack. "Tout a changé : le filtrage des informations et la censure, une actualité dans les nouveaux États-Unis d'Amérique ». Bulletin des bibliothèques de France, t. 47, n 2, 2002, p. 12-20.

Kessler Jack. « Religion et bibliothèques aux États-Unis ». Bulletin des bibliothèques de France, t. 48, n 6, 2003, p. 52-61.

Kibbee Jo. "Aux armes citoyens ! Les bibliothèques publiques françaises face à l'extrême droite ». Bulletin des bibliothèques de France, t. 49, n 6, 2004, p. 10-19.

Maack Mary N. "Americans in France: Cross-Cultural Exchange and the Diffusion of Innovations”. Journal of Library History, vol. 21, n² 2, Spring 1986, p. 315-332.

Poulain (Martine), « Retourner à Tocqueville pour comprendre l'histoire comparée des bibliothèques américaines et françaises au XIX siècle ", Bulletin des bibliothèques de France, t. 47, nº 5, 2002, p. 66-73.

Rayward W. Boyd. "Librarianship in the New World and the Old: Some Points of Contact”. Library Trends, July 1976. 
Shiflett Orvin L. “Clio’s Claim: the Role of Historical Research in Library and Information Service”. Library Trends, vol. 32, n 4, Spring 1984, p. 385-406.

Stone Elizabeth W. Historical Approach to American Library Development: A Chronological Chart. University of Illinois, Graduate School of Library Science, Occasional Papers, n 83, May 1967, p. 1-233.

Viot Pascal. « Enjeux et perspectives de l'engagement associatif ». Lecture publique et territoires : trente ans de mutations en BDP. Presses de l'enssib, 2005.

Wiegand Wayne A. "Tunnel Vision and Blind Spots: What the Past Tells us about the Present; Reflections on the Twentieth-Century History of American Librarianship". Library Quarterly, n 1, January 1999, p. 1-32.

\section{CORPUS DE DOCUMENTS PRIMAIRES}

\section{Monographies}

$\mathrm{ABF}$. Le métier de bibliothécaire : cours élémentaire de formation professionnelle. Promodis-Éditions du Cercle de la Librairie, 1968-2003.

Actes du colloque Lecture et bibliothèques publiques, Hénin-Beaumont, 20-21 novembre 1981. ORCEP, 1982.

ALA. Annual Conference Proceedings (puis) Council Minutes, 1946-2004.

Beckerman Edwin. Politics and the American Public Library: Creating Political Support for Library Goals. The Scarecrow Press, 1996.

Berelson Bernard, with the assistance of Lester Asheim. The Library's Public: A Report of the Public Library Inquiry. Columbia University Press, 1949.

Berninghausen David K. The Flight for Reason: Essays on Intellectual Freedom. ALA, 1975.

Les bibliothèques en France, [rapport Vandevoorde]. Dalloz, 1982.

Les bibliothèques en France, 1991-1997. Sous la dir. de Dominique Arot. Éditions du Cercle de la Librairie, 1998.

Books, Bricks \& Bytes: Libraries in the Twenty-First Century, ed. by Stephen R. Graubard \& Paul LeClerc. Transaction Publishers, 1999.

D'Elia George. The Roles of the Public Library in Society: The Results of a National Survey. Final Report. Urban Libraries Council, 1993. 
Garceau Oliver. The Public Library in the Political Process: A Report of the Public Library Inquiry. Columbia University Press, 1949.

Garrigoux Alice. La lecture publique en France. La Documentation française, 1972.

Gertzog Alice, Beckerman Edwin. Administration of the Public Library. The Scarecrow Press, 1994.

Gorman Michael. The Enduring Library: Technology, Tradition and the Quest for Balance. ALA, 2003.

Hafner Arthur. Democracy and the Public Library. Greenwood Press, 1993.

Hall Richard B. Financing Public Library Buildings. Neal-Schuman Publishers, 1994.

Keeping the Books: Public Library Financial Practices, ed. By Jane Robbins and Douglas L. Zweizig. Highsmith Press, 1992.

Leigh Robert D. The Public Library in the United States: The General Report of the Public Library Inquiry. Columbia University Press, 1950.

Libraries at Large: Tradition, Innovation and the National Interest, ed. By Douglas M. Knight and E. Shepley Nourse. Bowker Company, 1969.

Libraries \& Democracy: The Cornerstones of Liberty. Kranich Nancy ed. ALA, 2001.

Martin Lowell A. Library Response to Urban Change: A Study of the Chicago Public Library. ALA, 1969.

Martin Lowell A. Enrichment: A History of the Public Library in the United States in the 21st Century. The Scarecrow Press, 2003.

McCabe Ronald B. Civic Librarianship: Renewing the Social Mission of the Public Library. The Scarecrow Press, 2001.

McCook Katherine de la Peña. A Place at the Table. ALA, 2000.

Molz R. Kathleen. Federal Police and Library Support. The MIT Press, 1976.

Passeron Jean-Claude. Le raisonnement sociologique. Nathan, 1991.

Pungitore Verna L. Innovation and the Library: The Adoption of New Ideas in Public Libraries. Greenwood Press, 1995.

Regards croisés et perspectives : les bibliothèques publiques en Europe. BPI, 2000.

Richter Noë. La naissance de la lecture publique. Éditions de la Queue du chat, 1992. 
Robbins Jane. Citizen Participation and Public Library Policy. The Scarecrow Press, 1975.

Robine Nicole. Les jeunes travailleurs et la lecture. La Documentation française, 1984.

The Role of the Humanities in the Public Library. Broadus Robert N. ed. ALA, 1979.

Rose Ernestine. The Public Library in American Life. Columbia University Press, 1954.

Sager Donald J. Research Report on the American Public Library. OCLC, 1982.

Sager Donald J. Managing the Public Library. Knowledge Industry Publications, 1984.

Spencer Gwladys. The Chicago Public Library: Origins and Backgrounds. University of Chicago Press, 1943.

Thomison Dennis. A History of the American Library Association (1876-1972). ALA, 1978.

White Lawrence J. The Public Library in the 1980s: The Problems of Choice. Lexington Books, 1983.

Winther Barbara. They Like Noble Causes: How a Community Build a Library. Winslow Group, 2000.

Les articles consultés, trop nombreux, n’ont pas été repris ici. 


\section{SIGLES}

AAP : Association of American Publishers

AASL : American Association of School Librarians

ABF : Association des bibliothécaires français (Association des bibliothécaires de France, depuis 2006)

ACCES : Association culturelle contre les exclusions et les ségrégations

ACLU : American Civil Liberties Union

ACRL : Association of College and Research Librarians

ADBGV : Association des directeurs des bibliothèques des grandes villes

ADBU : Association des directeurs des bibliothèques universitaires et des personnels de direction et de la documentation

ALA : American Library Association

BBF : Bulletin des bibliothèques de France

BCP : Bibliothèque centrale de prêt, de la compétence des Conseils généraux

BDP : Bibliothèque départementale de prêt

BM : Bibliothèque municipale
BN : Bibliothèque nationale, devient Bibliothèque nationale de France en 1994

BnF : Bibliothèque nationale de France

BPI : Bibliothèque publique d'information (Paris)

CAFB : Certificat d'aptitude aux fonctions de bibliothécaire

CARD : Comité américain pour les régions dévastées

CIPA : Children's Internet Protection Act

COPA : Child Online Protection Act

CPPA : Child Pornography Prevention Act

DBLP : Direction des bibliothèques et de la lecture publique au ministère de la Culture et de la Communication, devient Direction du livre, puis Direction du livre et de la lecture (DLL).

DBMIST : Direction des bibliothèques, des musées et de l'information scientifique et technique au ministère de l'Enseignement supérieur et de la Recherche, créée en 1982, devient la Sous-direction des bibliothèques et de l'information scientifique (SDBIS), puis en 2009, la Mission de l'information scientifique et technique et du réseau documentaire (MISTRD). 
DLL : Direction du livre et de la lecture

DSB : Diplôme supérieur de bibliothèque, devient Diplôme de conservateur de bibliothèques (DCB)

EBSI : École de bibliothéconomie et de sciences de l'information (Montréal)

ENSB : École nationale supérieure des bibliothèques, devient en 1992 l'École nationale supérieure des sciences de l'information et des bibliothèques (ENSSIB)

FFCB : Fédération française de coopération entre bibliothèques

FIAB : Fédération internationale des associations de bibliothécaires et de bibliothèques, en anglais IFLA

FOLUSA : Friends of library of the United States of America

IFLA : International Federation of Library Associations and Institutions, en français FIAB

ISBN : International Standard Book Number

LCSA : Library Construction and Services Act, 1964

LSA : Library Services Act, 1956

LSTA : Library Services and Technology Act, 1996

NCES : National Center for Education Statistics

OCLC : Ohio College Library Center
PLA : Public Library Association

PLOS : Public Library of Science

PMI : Protection maternelle et infantile

USA Patriot Act: Uniting and Strengthening America by Providing Appropriate Tools Required to Intercept and Obstruct Terrorism, 2001 


\section{ANNEXES}

\section{TEXTES FONDATEURS DE L'AMERICAN LIBRARY ASSOCIATION}

\section{Library Bill of Rights}

The American Library Association affirms that all libraries are forums for information and ideas, and that the following basic policies should guide their services.

I. Books and other library resources should be provided for the interest, information, and enlightenment of all people of the community the library serves. Materials should not be excluded because of the origin, background, or views of those contributing to their creation.

II. Libraries should provide materials and information presenting all points of view on current and historical issues. Materials should not be proscribed or removed because of partisan or doctrinal disapproval.

III. Libraries should challenge censorship in the fulfillment of their responsibility to provide information and enlightenment.

IV. Libraries should cooperate with all persons and groups concerned with resisting abridgment of free expression and free access to ideas.

V. A person's right to use a library should not be denied or abridged because of origin, age, background, or views.

VI. Libraries which make exhibit spaces and meeting rooms available to the public they serve should make such facilities available on an equitable basis, regardless of the beliefs or affiliations of individuals or groups requesting their use.

Adopted June 18, 1948, by the ALA Council; amended February 2, 1961; January 23, 1980; inclusion of "age" reaffirmed January 23, 1996. 


\section{Déclaration des droits en bibliothèque}

(trad. Philippe Cantié, Au nom de l'antiterrorisme : les bibliothécaires américains face à l'USA Patriot Act, Presses de l'enssib, 2006)

L'American Library Association (ALA) affirme que toutes les bibliothèques sont des forums où s'échangent informations et idées et que, par conséquent, leurs services devraient être régis par les principes élémentaires suivants :

I. Les livres et autres ressources disponibles en bibliothèque se doivent de satisfaire l'intérêt, les besoins en information et le jugement de tous les membres de la communauté desservie. Il convient de n'exclure aucun document en raison de sa provenance, de son cadre de référence ou du point de vue de ses auteurs.

II. Les bibliothèques se doivent de fournir des documents et des informations présentant tous les points de vue sur les problèmes d'actualité comme sur les questions historiques. Il convient de ne proscrire ou retirer aucun document pour cause de désaccords politiques ou doctrinaux.

III. Les bibliothèques se doivent de lutter contre la censure dans l'accomplissement de leur mission qui consiste à fournir des documents et à permettre ainsi une meilleure compréhension du monde.

IV. Les bibliothèques se doivent de coopérer avec tous groupes ou personnes qui se préoccupent de défendre la liberté d'expression et la liberté d'accès aux idées.

V. Le droit d'un individu à utiliser une bibliothèque ne saurait être nié ou contesté pour des raisons d'origine ethnique ou sociale, d'âge ou d'opinion.

VI. Les bibliothèques qui proposent des espaces d'exposition et des salles de réunion au public qu'elles desservent doivent mettre ces équipements à disposition de manière équitable, sans tenir compte de l'opinion ni de l'appartenance des individus ou groupes qui en sollicitent l'usage. 


\section{The Freedom to Read Statement}

(adopté conjointement avec the Association of American Publishers en 1953)

The freedom to read is essential to our democracy. It is continuously under attack. Private groups and public authorities in various parts of the country are working to remove or limit access to reading materials, to censor content in schools, to label "controversial" views, to distribute lists of "objectionable" books or authors, and to purge libraries. These actions apparently rise from a view that our national tradition of free expression is no longer valid; that censorship and suppression are needed to counter threats to safety or national security, as well as to avoid the subversion of politics and the corruption of morals. We, as individuals devoted to reading and as librarians and publishers responsible for disseminating ideas, wish to assert the public interest in the preservation of the freedom to read.

Most attempts at suppression rest on a denial of the fundamental premise of democracy: that the ordinary individual, by exercising critical judgment, will select the good and reject the bad. We trust Americans to recognize propaganda and misinformation, and to make their own decisions about what they read and believe. We do not believe they are prepared to sacrifice their heritage of a free press in order to be "protected" against what others think may be bad for them. We believe they still favor free enterprise in ideas and expression.

These efforts at suppression are related to a larger pattern of pressures being brought against education, the press, art and images, films, broadcast media, and the Internet. The problem is not only one of actual censorship. The shadow of fear cast by these pressures leads, we suspect, to an even larger voluntary curtailment of expression by those who seek to avoid controversy or unwelcome scrutiny by government officials.

Such pressure toward conformity is perhaps natural to a time of accelerated change. And yet suppression is never more dangerous than in such a time of social tension. Freedom has given the United States the elasticity to endure strain. Freedom keeps open the path of novel and creative solutions, and enables change to come by choice. Every silencing of a heresy, every enforcement of an orthodoxy, diminishes the toughness and resilience of our society and leaves it the less able to deal with controversy and difference.

Now as always in our history, reading is among our greatest freedoms. The freedom to read and write is almost the only means for making generally available ideas or manners of expression that can initially command only a small audience. The written word is the natural medium for the new idea and the untried voice from which come the original contributions to social growth. It is essential to the extended discussion that serious thought requires, and to the accumulation of knowledge and ideas into organized collections.

We believe that free communication is essential to the preservation of a free society and a creative culture. We believe that these pressures toward conformity present the danger of limiting the range and variety of inquiry and expression on 


\section{Déclaration sur le droit de lire}

(trad. Philippe Cantié, Au nom de l'antiterrorisme : les bibliothécaires américains face à l'USA Patriot Act, Presses de l'enssib, 2006)

La liberté de lecture est essentielle à notre démocratie. Elle subit pourtant des attaques en permanence. Groupes privés et autorités publiques s'efforcent aux quatre coins du pays de supprimer ou de limiter l'accès à certains documents, de censurer des contenus enseignés dans les écoles, de mettre à l'index des opinions "non conformistes », de diffuser des listes d'ouvrages ou d'auteurs « condamnables », d'épurer les bibliothèques. Ces actes découlent de l'opinion selon laquelle notre tradition nationale de liberté d'expression aurait perdu toute validité. La censure et l'élimination de certains documents seraient nécessaires pour faire face aux menaces qui pèsent sur la sécurité du pays, mais aussi à la subversion de notre régime politique et à la corruption de nos mœurs. En tant qu'individus dévoués à la cause de la lecture et responsables, à titre d'éditeurs ou de bibliothécaires, de la diffusion des idées, nous souhaitons affirmer qu'il est de l'intérêt de tous de sauvegarder la liberté de lecture.

La plupart des tentatives de censure reposent sur le déni des prémices de la démocratie, à savoir que tout individu, par l'exercice de son jugement critique, est en mesure de choisir le bien et de rejeter le mal. Nous avons confiance dans la capacité des Américains à déceler la propagande et la désinformation, ainsi qu’à faire leur propre choix quant à ce qu'il convient de lire et de penser. Nous ne croyons pas qu'ils soient prêts à sacrifier l'héritage d'une presse libre afin de «se protéger » de ce que d'autres estiment être potentiellement mauvais pour eux. Nous pensons qu'ils sont toujours favorables à la libre entreprise dans le domaine des idées et de leur mode d'expression.

Ces tentatives de censure font partie d'un ensemble plus large de pressions qui s'exercent sur l'éducation, la presse, les arts, les images, les films, l'audiovisuel et l'Internet. Le problème ne concerne pas seulement les cas de censure effective. La crainte qu'engendrent ces pressions conduit, on s'en doute, à une autocensure chez ceux qui cherchent à éviter toute controverse ou à se dérober au regard inquisiteur des agents du gouvernement.

La pression du conformisme est peut-être naturelle à une époque où s'accélère le rythme du changement. Pourtant la censure n'est jamais plus dangereuse qu'en période de tension sociale. La liberté a donné aux États-Unis l'élasticité nécessaire pour résister aux tensions. Grâce à elle, le chemin qui conduit à la création de solutions nouvelles reste ouvert et le changement résulte de choix conscients. Le fait de réduire au silence toute opinion hérétique ou d'œuvrer pour l'orthodoxie diminue la fermeté de notre société et sa capacité à rebondir. Notre société est alors moins à même de faire face aux différences et à la discorde.

Aujourd'hui comme hier, le droit de lire compte parmi nos libertés les plus éminentes. La liberté de lire et d'écrire est quasiment le seul moyen de faire connaître des idées ou des manières d'expression qui au départ ne recueillent souvent 
which our democracy and our culture depend. We believe that every American community must jealously guard the freedom to publish and to circulate, in order to preserve its own freedom to read. We believe that publishers and librarians have a profound responsibility to give validity to that freedom to read by making it possible for the readers to choose freely from a variety of offerings.

The freedom to read is guaranteed by the Constitution. Those with faith in free people will stand firm on these constitutional guarantees of essential rights and will exercise the responsibilities that accompany these rights.

We therefore affirm these propositions:

1. It is in the public interest for publishers and librarians to make available the widest diversity of views and expressions, including those that are unorthodox, unpopular, or considered dangerous by the majority.

Creative thought is by definition new, and what is new is different. The bearer of every new thought is a rebel until that idea is refined and tested. Totalitarian systems attempt to maintain themselves in power by the ruthless suppression of any concept that challenges the established orthodoxy. The power of a democratic system to adapt to change is vastly strengthened by the freedom of its citizens to choose widely from among conflicting opinions offered freely to them. To stifle every nonconformist idea at birth would mark the end of the democratic process. Furthermore, only through the constant activity of weighing and selecting can the democratic mind attain the strength demanded by times like these. We need to know not only what we believe but why we believe it.

2. Publishers, librarians, and booksellers do not need to endorse every idea or presentation they make available. It would conflict with the public interest for them to establish their own political, moral, or aesthetic views as a standard for determining what should be published or circulated.

Publishers and librarians serve the educational process by helping to make available knowledge and ideas required for the growth of the mind and the increase of learning. They do not foster education by imposing as mentors the patterns of their own thought. The people should have the freedom to read and consider a broader range of ideas than those that may be held by any single librarian or publisher or government or church. It is wrong that what one can read should be confined to what another thinks proper.

3. It is contrary to the public interest for publishers or librarians to bar access to writings on the basis of the personal history or political affiliations of the author.

No art or literature can flourish if it is to be measured by the political views or private lives of its creators. No society of free people can flourish that draws up lists of writers to whom it will not listen, whatever they may have to say. 
que peu de suffrages. La parole écrite est le médium naturel pour toute idée ou voix nouvelle qui contribue de manière originale au développement de la société. L'écrit permet de discuter de manière approfondie toute réflexion sérieuse et de classer en ensembles organisés l'accumulation des idées et des connaissances.

Nous estimons que la liberté de communiquer est essentielle au maintien d'une société libre et d'une société créatrice. Nous pensons que la pression du conformisme sous ses multiples formes risque de limiter, à la fois dans l'étendue et la variété, notre liberté d'examen et notre liberté d'expression sur lesquelles reposent notre culture et notre démocratie. Nous pensons que toute communauté américaine doit protéger jalousement sa liberté d'édition et de diffusion afin de préserver son propre droit de lire. Nous croyons que les éditeurs et les bibliothécaires ont une grande responsabilité : faire en sorte que les lecteurs puissent choisir librement à partir d'une offre variée.

Le droit de lire est garanti par la Constitution. Ceux qui ont foi en un peuple libre ne transigeront pas sur les garanties constitutionnelles relatives à ce droit essentiel et exerceront les responsabilités qui l'accompagnent.

Nous avançons par conséquent les propositions suivantes :

1. Il est dans l'intérêt du public que les éditeurs et les bibliothécaires diffusent la plus grande diversité possible d'opinions et modes d'expression, $y$ compris ceux qui sont hétérodoxes, impopulaires ou considérés comme dangereux par la majorité.

La pensée créatrice est par définition nouvelle, et ce qui est nouveau est forcément différent. Le porteur de toute idée nouvelle est un rebelle jusqu'à ce que l'idée soit affinée et mise à l'épreuve. Les systèmes totalitaires s'efforcent de se maintenir au pouvoir par l'élimination drastique de tout concept qui bouleverse l'orthodoxie en vigueur. La capacité du système démocratique à s'adapter au changement est considérablement renforcée par la liberté des citoyens de choisir parmi le large éventail d'opinions contradictoires qui leur sont librement proposées. Étouffer dès sa naissance toute idée non conformiste reviendrait à sonner le glas du processus démocratique. De plus, ce n'est que par un travail constant de critique et de sélection que l'esprit démocratique peut acquérir la vigueur requise par les temps que nous vivons. Il est nécessaire de savoir non seulement ce en quoi nous croyons mais aussi pourquoi nous y croyons.

2. Il n'est pas nécessaire que les éditeurs, les bibliothécaires et les libraires cautionnent toutes les idées qu'ils mettent à portée du public. Il serait contraire à l'intérêt public qu'ils se basent sur le seul critère de leurs opinions personnelles dans le domaine politique, moral ou esthétique pour déterminer ce qui doit être publié ou diffusé.

Les éditeurs et les bibliothécaires, qui contribuent à mettre à disposition de tous les connaissances et les idées nécessaires au développement de l'esprit et du savoir, sont au service du processus éducatif. Ils ne favori- 
4. There is no place in our society for efforts to coerce the taste of others, to confine adults to the reading matter deemed suitable for adolescents, or to inhibit the efforts of writers to achieve artistic expression.

To some, much of modern expression is shocking. But is not much of life itself shocking? We cut off literature at the source if we prevent writers from dealing with the stuff of life. Parents and teachers have a responsibility to prepare the young to meet the diversity of experiences in life to which they will be exposed, as they have a responsibility to help them learn to think critically for themselves. These are affirmative responsibilities, not to be discharged simply by preventing them from reading works for which they are not yet prepared. In these matters values differ, and values cannot be legislated; nor can machinery be devised that will suit the demands of one group without limiting the freedom of others.

5. It is not in the public interest to force a reader to accept the prejudgment of a label characterizing any expression or its author as subversive or dangerous.

The ideal of labeling presupposes the existence of individuals or groups with wisdom to determine by authority what is good or bad for others. It presupposes that individuals must be directed in making up their minds about the ideas they examine. But Americans do not need others to do their thinking for them.

6. It is the responsibility of publishers and librarians, as guardians of the people's freedom to read, to contest encroachments upon that freedom by individuals or groups seeking to impose their own standards or tastes upon the community at large; and by the government whenever it seeks to reduce or deny public access to public information.

It is inevitable in the give and take of the democratic process that the political, the moral, or the aesthetic concepts of an individual or group will occasionally collide with those of another individual or group. In a free society individuals are free to determine for themselves what they wish to read, and each group is free to determine what it will recommend to its freely associated members. But no group has the right to take the law into its own hands, and to impose its own concept of politics or morality upon other members of a democratic society. Freedom is no freedom if it is accorded only to the accepted and the inoffensive. Further, democratic societies are more safe, free, and creative when the free flow of public information is not restricted by governmental prerogative or self-censorship.

7. It is the responsibility of publishers and librarians to give full meaning to the freedom to read by providing books that enrich the quality and diversity of thought and expression. By the exercise of this affirmative respon- 
sent pas l'éducation en imposant, en tant que mentors, leurs propres schémas de pensée. Le public doit jouir de la liberté de lire et examiner une gamme d'idées dont l'empan dépasse l'opinion d'un seul bibliothécaire ou éditeur, du gouvernement ou de l'Église. Il n'est pas normal que les lectures d'une personne se limitent aux lectures qu'une autre personne estime convenables.

3. Il est contraire à l'intérêt public que des éditeurs ou des bibliothécaires empêchent l'accès à des écrits en prenant pour motif l'histoire personnelle ou les convictions politiques de leur auteur.

Aucun art, aucune littérature ne peuvent s'épanouir s'ils sont jaugés à l'aune des opinions politiques ou de la vie privée des créateurs. Aucune société libre ne peut s'épanouir si elle dresse des listes d'écrivains dont le discours est proscrit, quels qu'en soient la teneur et le contenu.

4. Notre société ne saurait approuver ceux qui s'efforcent de contraindre les goûts d'autrui, de cantonner un public adulte à des lectures pour adolescents ou d'empêcher les écrivains de s'exprimer sur le plan artistique.

Nombreux sont ceux qui sont choqués par les formes modernes d'expression. Mais la vie elle-même n'est-elle pas souvent choquante? Nous tarissons la source de toute littérature si nous empêchons les écrivains de parler de ce qui fait la vie. Les parents et les éducateurs ont pour responsabilité de préparer les jeunes à affronter la diversité des expériences auxquelles ils auront à faire face au cours de leur vie, ainsi que de leur apprendre à penser par eux-mêmes en faisant preuve de sens critique. Il s'agit là de responsabilités positives dont on ne peut se décharger en empêchant simplement les jeunes de lire des œuvres auxquelles ils ne sont point encore préparés. Dans ce genre d'affaires, les valeurs diffèrent et il est impossible de légiférer sur les valeurs. On ne peut concevoir de dispositif qui satisfasse les exigences d'un groupe sans limiter la liberté d'autres groupes.

5. Il n'est pas dans l'intérêt public de contraindre le lecteur à accepter le jugement a priori d'une signalisation qui caractériserait une forme d'expression ou un auteur comme dangereux ou subversif.

Un tel étiquetage supposerait l'existence d'individus ou de groupes suffisamment avisés pour déterminer autoritairement ce qui est bon ou mauvais pour autrui. Il supposerait que les individus ont besoin d'être guidés pour se prononcer sur les idées qu'ils examinent. Mais les Américains n'ont besoin de personne pour penser à leur place.

6. Il est de la responsabilité des éditeurs et des bibliothécaires, en tant que gardiens de la liberté de lire, de lutter contre les atteintes à cette liberté de la part d'individus ou de groupes qui cherchent à imposer leurs propres goûts ou critères à l'ensemble de la collectivité ; ou de la part du gouver- 
sibility, they can demonstrate that the answer to a "bad" book is a good one, the answer to a "bad" idea is a good one.

The freedom to read is of little consequence when the reader cannot obtain matter fit for that reader's purpose. What is needed is not only the absence of restraint, but the positive provision of opportunity for the people to read the best that has been thought and said. Books are the major channel by which the intellectual inheritance is handed down, and the principal means of its testing and growth. The defense of the freedom to read requires of all publishers and librarians the utmost of their faculties, and deserves of all Americans the fullest of their support.

We state these propositions neither lightly nor as easy generalizations. We here stake out a lofty claim for the value of the written word. We do so because we believe that it is possessed of enormous variety and usefulness, worthy of cherishing and keeping free. We realize that the application of these propositions may mean the dissemination of ideas and manners of expression that are repugnant to many persons. We do not state these propositions in the comfortable belief that what people read is unimportant. We believe rather that what people read is deeply important; that ideas can be dangerous; but that the suppression of ideas is fatal to a democratic society. Freedom itself is a dangerous way of life, but it is ours.

This statement was originally issued in May of 1953 by the Westchester Conference of the American Library Association and the American Book Publishers Council, which in 1970 consolidated with the American Educational Publishers Institute to become the Association of American Publishers.

Adopted June 25, 1953, by the ALA Council and the AAP Freedom to Read Committee; amended January 28, 1972; January 16, 1991; July 12, 2000; June 30, 2004. 
nement à chaque fois qu'il cherche à réduire ou à refuser l'accès du public à des informations publiques.

Il est inévitable que dans le compromis inhérent au processus démocratique, les positions politiques, morales, esthétiques d'un individu ou d'un autre entrent de temps en temps en contradiction avec celles d'un autre individu ou d'un autre groupe. Dans une société, les individus sont libres de faire leur choix de lectures, et chaque groupe est libre de recommander à ses membres les lectures qu'elle a choisies. Mais aucun groupe n'a le droit de faire la loi et d'imposer ses idées politiques ou morales sur les autres membres d'une société démocratique. La liberté est un vain mot si elle n'est accordée qu'aux citoyens les plus conformistes ou inoffensifs. De plus, les sociétés démocratiques ne sont point libres, créatrices et en sécurité lorsque l'autocensure ou les prérogatives du gouvernement empêchent la libre circulation d'informations publiques.

7. Il est de la responsabilité des éditeurs et des bibliothécaires de donner tout son sens à la liberté de lire en procurant des ouvrages qui enrichissent la qualité et la diversité des idées et de leur expression. Ces professionnels peuvent, par l'exercice de cette responsabilité positive, faire la preuve que la réponse à un " mauvais » livre est un bon "livre " et que la réponse à une " mauvaise idée » est une bonne idée.

La liberté de lire n'est que de peu d'utilité si le lecteur ne peut se procurer une matière qui correspond à ce qu'il recherche. Ce qui importe, ce n'est pas seulement l'absence de contrainte mais la possibilité effective pour le lecteur de lire le meilleur de ce qui a été dit et pensé. Les livres sont le canal principal par lequel se transmet l'héritage intellectuel, le moyen principal de le mettre à l'épreuve et de le faire fructifier. La défense de la liberté de lire requiert l'entière mobilisation des éditeurs et bibliothécaires et mérite le soutien absolu de tous les Américains.

Les propositions qui précèdent ne sont pas à prendre à la légère. Il ne s'agit pas non plus d'évidences très générales. Nous exprimons solennellement à travers elles notre croyance en la valeur de l'écrit. Il importe de reconnaître et de garantir la liberté de l'écrit à cause de son immense variété et de son utilité. Nous avons conscience du fait que l'application de ces propositions risque d'entraîner la diffusion d'idées et de formes d'expression qui paraîtront révoltantes à beaucoup. Nous n'exprimons pas ces propositions en nous abritant derrière l'idée rassurante que ce qui se lit n'a aucune importance. Nous croyons au contraire à l'importance de ce qui se lit. Nous croyons que certaines idées sont dangereuses mais que la censure de ces idées serait un coup fatal porté à la démocratie. La liberté est un mode de vie risqué mais c'est le nôtre. 


\section{Ways Libraries Are Good for the Country}

(a 2000 revision of the list that originally appeared in American Libraries in December 1995)

MOST AMERICANS KNOW what they can expect from a library. And librarians know what it takes to provide comprehensive access to every recorded detail of human existence. It takes support.

Libraries are ready when they are needed, ready to enrich our minds and defend our right to know, just as other institutions protect our safety and property. Without sound minds, however, the American dream of safe streets and secure homes will never be fulfilled.

Libraries safeguard our freedom and keep democracy healthy. To library advocates everywhere - friends, trustees, board members, patrons, and volunteers American Libraries offers this gift of 12 ideals toward which we strive. It will take all of us, in a spirit of pride and freedom, to maintain libraries as a living reality in a free nation into the 21 st century.

1. Libraries inform citizens. Democracy vests supreme power in the people. Libraries make democracy work by providing access to information so that citizens can make the decisions necessary to govern themselves. The public library is the only institution in American society whose purpose is to guard against the tyrannies of ignorance and conformity, and its existence indicates the extent to which a democratic society values knowledge, truth, justice, books, and culture.

2. Libraries break down boundaries. Libraries provide free family literacy programs for low-literate, illiterate, and non-English-speaking people. In addition, hundreds of librarians across America lead outreach programs that teach citizenship and develop multilingual and multicultural materials for their patrons. Libraries serve the homebound elderly, prisoners, and other institutionalized individuals, the homeless, and the blind and hearing-impaired.

3. Libraries level the playing field. Economists have cited a growing income inequity in America, with the gap between the richest and poorest citizens becoming wider year by year. By making all its resources equally available to all members of its community, regardless of income, class, or other factors, the library levels the playing field. Once users have access to the library's materials, they have the opportunity to level the playing field outside the library by learning to read, gaining employment, or starting a business.

4. Libraries value the individual. Library doors swing open for independent thinking without prejudgment. Libraries offer alternatives to the manipulations of commercialism, from the excellence of public-television productions to the freethinking 


\section{Douze raisons pour lesquelles les bibliothèques sont bonnes pour le pays}

(version 2000 de la liste originale publiée dans American Libraries en décembre 1995)

La plupart des Américains savent ce qu'ils peuvent attendre d'une bibliothèque. Et les bibliothécaires savent ce que cela demande que de fournir un accès attentif à chaque moment mémorable de la vie humaine. Cela demande du soutien.

Les bibliothèques sont prêtes quand on a besoin d'elles, prêtes à enrichir nos esprits et à défendre notre droit de savoir, exactement comme d'autres institutions protègent notre sécurité et nos biens. Quoi qu'il en soit, sans des esprits formés, le rêve américain d'avoir des rues tranquilles et des domiciles sûrs ne sera jamais atteint.

Les bibliothèques protègent notre liberté et gardent notre démocratie forte. Pour les soutiens des bibliothèques, partout, amis des bibliothèques, administrateurs, membres des conseils, clients et volontaires, American Libraries offre ces 12 idéaux que nous nous efforçons d'atteindre. On aura besoin de nous tous, dans un esprit de fierté et de liberté, pour continuer à faire des bibliothèques une réalité vivante dans une nation libre à l'aube du XXI siècle.

1. Les bibliothèques informent les citoyens. La démocratie met le pouvoir suprême dans les mains du peuple. Les bibliothèques contribuent à la démocratie en procurant l'accès à l'information pour que les citoyens puissent prendre les décisions nécessaires à leur propre gouvernement. La Public Library est la seule institution dans la société américaine dont le but est de protéger contre la tyrannie de l'ignorance et du conformisme et son existence est la mesure de l'importance qu'une société démocratique accorde à la connaissance, à la vérité, à la justice, aux livres et à la culture.

2. Les bibliothèques abolissent les frontières. Les bibliothèques procurent gratuitement des programmes de formation pour les peu éduqués, pour les illettrés et pour les personnes qui ne parlent pas anglais. En outre, des centaines de bibliothécaires à travers l'Amérique mènent des programmes hors-les-murs pour enseigner la citoyenneté et développer des collections multilingues et multiculturelles pour leurs clients. Les bibliothèques servent les personnes âgées grabataires, les détenus, et d'autres individus au statut spécifique, les sans-abris, les aveugles et les personnes malentendantes.

3. Les bibliothèques élèvent le niveau. Les économistes ont noté une inégalité croissante en Amérique, l'écart entre les plus riches et les plus pauvres s'élargissant d'année en année. En rendant ses ressources équitablement accessibles à tous les membres de la population, quels que soient leurs revenus, leur classe ou tout autre critère, la bibliothèque élève le niveau. Quand les usagers ont accès aux 
of renegade publishers and the vision of poets and artists outside the mainstream business of art and literature.

5. Libraries nourish creativity. In the library we are all children. By stimulating curiosity - parent to the twin forces of creativity and imagination - even the most focused and specialized library serves the purpose of lifting the mind beyond its horizons. Libraries store ideas that may no longer work but can serve as the raw material that, cross-fertilized in the innovative mind, may produce answers to questions not yet asked.

6. Libraries open kids' minds. Bringing children into a library can transport them from the commonplace to the extraordinary. From story hours for preschoolers to career planning for high schoolers, children's librarians make a difference because they care about the unique developmental needs of every individual who comes to them for help. Children get a handle on personal responsibility by holding a library card of their own, a card that gives them access to new worlds in books, videos, audiotapes, computers, games, toys, and more.

7. Libraries return high dividends. What do Gallo wines, the I Can't Believe It's Yogurt chain, and billboard-sign giant Metromedia have in common? Libraries made millionaires out of each of these companies' grateful owners by providing crucial start-up information when they were no more than wannabe business titans. Libraries are there to help people with more personal goals, too. The seed money expended for these and other success stories? Less than $\$ 20$ per capita per year in tax dollars.

8. Libraries build communities. No narrow definition of community will work in a library. Each community has its libraries and its special collections. Libraries validate and unify; they save lives, literally and by preserving the record of those lives. Community-building means libraries link people with information. Librarians have become experts at helping others navigate the Internet. Before there was talk of cyberspace, there were libraries, paving the way for the superhighway.

9. Libraries make families friendlier. The American family's best friend, the library, offers services guaranteed to home coping skills. Homework centers, literacy training, parenting materials, after-school activities, summer reading programs, outreach - like the families they serve, libraries everywhere are adapting to meet new challenges.

10. Libraries offend everyone. Children's librarian Dorothy Broderick contends that every library in the country ought to have a sign on the door reading : "This library has something offensive to everyone. If you are not offended by something we own, please complain." This willingness and duty to offend connotes a tolerance and a willingness to look at all sides of an issue that would be good for the 
documents de la bibliothèque, ils peuvent élever leur niveau hors de la bibliothèque en apprenant à lire, en trouvant du travail ou en créant une affaire.

4. Les bibliothèques valorisent les individus. Les bibliothèques ouvrent leurs portes à la pensée indépendante, sans préjugés. Les bibliothèques proposent une alternative à l'offre commerciale, depuis l'excellence des productions de la télévision publique jusqu'à la pensée libre des petits éditeurs et à la vision des poètes et des artistes en dehors de la production courante de l'art et de la littérature.

5. Les bibliothèques encouragent la création. Dans la bibliothèque, nous sommes tous des enfants. En stimulant la curiosité - qui nourrit les forces jumelles de la création et de l'imagination - même les bibliothèques les plus spécialisées contribuent à l'objectif d'élever l'esprit au-delà de son horizon. Les bibliothèques conservent des idées qui peuvent ne plus marcher mais qui peuvent servir comme matériaux de base qui, par l'intermédiaire d'un esprit innovant, peuvent fournir des réponses à des questions qu'on ne s'était encore jamais posées.

6. Les bibliothèques ouvrent l'esprit des enfants. Amener des enfants à la bibliothèque peut les transporter d'un endroit ordinaire à un endroit extraordinaire. Depuis l'heure du conte pour les tout-petits jusqu'à l'orientation scolaire pour les lycéens, les bibliothécaires pour la jeunesse apportent leur différence parce qu'ils ne se soucient que du développement individuel de celui qui leur demande de l'aide. Les enfants sont personnellement responsables de leur propre carte de bibliothèque, une carte qui leur donne accès à d'autres mondes dans les livres, les vidéos, les cassettes, les ordinateurs, les jeux, les jouets, etc.

7. Les bibliothèques rapportent gros. Qu'est-ce que les vins Gallo, la chaîne I Can't Believe It's Yogurt ou les panneaux d'affichage Metromedia ont en commun ? Les bibliothèques ont rendu millionnaires leurs propriétaires reconnaissants en leur apportant des informations cruciales pour démarrer à un moment où ils n'étaient que des géants de l'industrie en puissance. Les bibliothèques sont là aussi pour aider les gens qui ont des projets personnels. L’argent de départ dépensé pour ces success stories? Moins de 20 dollars d'impôt par personne et par an.

8. Les bibliothèques construisent des communautés. Aucune définition étroite de ce qu'est une communauté ne fonctionne dans une bibliothèque. Chaque communauté a ses bibliothèques et ses collections. Les bibliothèques servent de liens ; elles sauvent des vies, littéralement et en préservant la mémoire de ces vies. Construire des communautés signifie relier les gens et l'information. Les bibliothécaires sont devenus des experts pour aider les gens à surfer sur Internet. Avant même qu'on parle de cyberespace, les bibliothèques étaient là, en train de paver les autoroutes de l'information. 
nation in any context; it is particularly valuable when combined with the egalitarianism and openness that characterize libraries.

11. Libraries offer sanctuary. Like synagogues, churches, mosques, and other sacred spaces, libraries can create a physical reaction, a feeling of peace, respect, humility, and honor that throws the mind wide open and suffuses the body with a near-spiritual pleasure. But why? Perhaps it is because in the library we are answerable to no one; alone with our private thoughts, fantasies, and hopes, we are free to nourish what is most precious to us with the silent companionship of others we do not know.

12. Libraries preserve the past. Libraries preserve the record; a nation, a culture, a community that does not understand its own past is mired in its own mistakes. Libraries enable us to communicate through distance and time with the living and the dead. It is a miracle kept available by the meticulous sorting, storing, indexing, and preservation that still characterizes library work - work that will carry, in the electronic environment, challenges and a price tag yet unknown.

Adapted from "12 Ways Libraries Are Good for the Country", American Libraries, 26 (December 1995) : 1113 - 19. 
9. Les bibliothèques aident les familles. La meilleure amie de la famille américaine, la bibliothèque, offre des services de qualité pour mieux se débrouiller à la maison. Les centres d'aide aux devoirs, les cours d'alphabétisation, les documents pour l'éducation des enfants, les activités post-scolaires, les ateliers de lecture l'été, les activités hors-les-murs - comme les familles qu'elles desservent, les bibliothèques, partout, s'adaptent et rendent de nouveaux services.

10. Les bibliothèques choquent tout le monde. La bibliothécaire pour enfants Dorothy Broderick soutient que chaque bibliothèque du pays devrait avoir sur sa porte une affiche où l'on pourrait lire : « Cette bibliothèque possède quelque chose de choquant pour chacun de vous. Si vous n'êtes choqué par rien de ce que nous possédons, merci de vous en plaindre. » Ce souhait et ce devoir de choquer correspondent à la tolérance et à la volonté d'examiner tous les aspects d'un sujet, ce qui est toujours bon pour la nation ; c'est particulièrement important lorsque cela se combine avec le souci d'égalité et la volonté d'ouverture qui caractérisent les bibliothèques.

11. Les bibliothèques sont un sanctuaire. Comme les synagogues, les églises, les mosquées et autres lieux de culte, les bibliothèques peuvent créer une réaction physique, un sentiment de paix, de respect, d'humilité et d'honneur qui ouvre largement l'esprit et procure un plaisir presque spirituel. Pourquoi ? Peut-être parce que dans les bibliothèques nous ne sommes guidés par personne. Chacun de notre côté, avec nos propres pensées, fantaisies et espoirs, nous sommes libres de nourrir ce qui nous est le plus précieux, dans la compagnie silencieuse de gens que nous ne connaissons pas.

12. Les bibliothèques conservent le passé. Les bibliothèques conservent la mémoire ; une nation, une culture, une communauté qui ne comprend pas son propre passé est enfermée dans ses propres erreurs. Les bibliothèques nous permettent, à travers l'espace et le temps, de dialoguer avec les vivants et les morts. C'est un miracle que rend possible le méticuleux travail de collecte, de classement, d'indexation, de conservation qui caractérise encore l'activité des bibliothèques - activité qui, dans l'environnement numérique, sera un défi et aura un coût encore inconnu.

Adapté de «Douze raisons pour lesquelles les bibliothèques sont bonnes pour le pays », American Libraries, n² 26, December 1995, p. 1113-1119. 


Secrétariat d'édition :

Silvia Ceccani

Mise en page :

Alexandre Bocquier

Conception graphique :

atelier Perluette, 69001 Lyon

$<$ http://www.perluette-atelier.com >

Achevé d'imprimer en février 2010

imprimerie Chirat

dépôt légal : $1^{\mathrm{er}}$ semestre 2010 
La réflexion sur le modèle de bibliothèque entamée avec Quel modèle de bibliothèque ? (Presses de l'enssib, 2008) se poursuit par une analyse comparée des modèles français et américain de bibliothèque publique.

Sont ici analysés les modalités de création de ces bibliothèques, leur rapport aux pouvoirs, leur place dans la société, leurs fonctions et leurs fonctionnements. Un chapitre complète l'analyse avec une présentation des deux grandes associations professionnelles (Association des bibliothécaires de France, American Library Association).

Une place importante est consacrée à l'analyse comparée des fondements et des objectifs de chacun de ces modèles : aux États-Unis, l'accent mis sur l'accès libre à l'information et sur la fonction éducative de la Public Library; en France, sur l'offre de collections et services culturels et sur l'accès à tous (la démocratisation culturelle).

Au-delà d'un socle commun de valeurs et de projets, cet ouvrage souligne l'écart entre les choix des établissements de part et d'autre de l'Atlantique, leur rapport au savoir, à l'information et à la censure, et les représentations que l'histoire récente des uns et des autres ont pu créer dans l'imaginaire collectif.

Anne-Marie Bertrand, conservateur général des bibliothèques, a dirigé les bibliothèques de Roubaix et de Nantes, puis le service Études et recherche de la Bibliothèque publique d'information. Elle est aujourd'hui directrice de l'enssib. 